\title{
Clinical Practice Guidelines for Antimicrobial Prophylaxis in Surgery
}

\author{
Dale W. Bratzler, E. Patchen Dellinger, ${ }^{2}$ Keith M. Olsen, ${ }^{3}$ Trish M. Perl, ${ }^{4}$ Paul G. Auwaerter, ${ }^{5}$ \\ Maureen K. Bolon, ${ }^{6}$ Douglas N. Fish, ${ }^{7}$ Lena M. Napolitano, ${ }^{8}$ Robert G. Sawyer, ${ }^{9}$ Douglas Slain, ${ }^{10}$ \\ James P. Steinberg, ${ }^{11}$ and Robert A. Weinstein ${ }^{12}$
}

T HESE GUIDELINES WERE developed jointly by the American Society of Health-System Pharmacists (ASHP), the Infectious Diseases Society of America (IDSA), the Surgical Infection Society (SIS), and the Society for Healthcare Epidemiology of America (SHEA). This work represents an update to the previously published ASHP Therapeutic Guidelines on Antimicrobial Prophylaxis in Surgery [1], as well as guidelines from IDSA and SIS [2,3]. The guidelines are intended to provide practitioners with a standardized approach to the rational, safe, and effective use of antimicrobial agents for the prevention of surgical site infections (SSIs) based on currently available clinical evidence and emerging issues.

Prophylaxis refers to the prevention of an infection and can be characterized as primary prophylaxis, secondary prophylaxis, or eradication. Primary prophylaxis refers to the prevention of an initial infection. Secondary prophylaxis refers to the prevention of recurrence or reactivation of a preexisting infection. Eradication refers to the elimination of a colonized organism to prevent the development of an infection. These guidelines focus on primary perioperative prophylaxis.

\section{Guidelines development and use}

Members of ASHP, IDSA, SIS, and SHEA were appointed to serve on an expert panel established to ensure the validity, reliability, and utility of the revised guidelines. The work of the panel was facilitated by faculty of the University of Pittsburgh School of Pharmacy and University of Pittsburgh Medical Center Drug Use and Disease State Management Program who served as contract researchers and writers for the project. Panel members and contractors were required to disclose any possible conflicts of interest before their appointment and throughout the guideline development process. Drafted documents for each surgical procedural section were reviewed by the expert panel and, once revised, were available for public comment on the ASHP website. After additional revisions were made to address reviewer comments, the final document was approved by the expert panel and the boards of directors of the above-named organizations.

\section{Strength of evidence and grading of recommendations}

The primary literature from the previous ASHP Therapeutic Guidelines on Antimicrobial Prophylaxis in Surgery [1] was reviewed together with the primary literature published between the date of the previous guidelines, 1999, and June 2010, identified by searches of MEDLINE, EMBASE, and the Cochrane Database of Systematic Reviews. Particular attention was paid to study design, with greatest credence given to randomized, controlled, double-blind studies. There is a limited number of adequately powered randomized controlled trials evaluating the efficacy of antimicrobial prophylaxis in surgical procedures. Guidelines development included consideration of the following characteristics: validity, reliability, clinical applicability, flexibility, clarity, and a multidisciplinary nature as consistent with ASHP's philosophy on therapeutic guidelines [4]. The limitations of the evidence base are noted within each individual procedure section of the guidelines. Published guidelines with recommendations by experts in a procedure area (e.g., American College of Obstetricians and

\footnotetext{
${ }^{1}$ College of Public Health, and Professor, College of Medicine, Oklahoma University Health Sciences Center, Oklahoma City, Oklahoma.

${ }^{2}$ Department of Surgery, and Chief, Division of General Surgery, University of Washington, Seattle, Washington.

${ }^{3}$ Nebraska Medical Center, Omaha, Nebraska.

${ }^{4}$ Pathology, and Epidemiology, Johns Hopkins University, and Senior Epidemiologist, The Johns Hopkins Health System, Baltimore, Maryland. ${ }^{5}$ Division of Infectious Diseases, School of Medicine, Johns Hopkins University, Baltimore, Maryland.

${ }^{6}$ Division of Infectious Diseases, Feinberg School of Medicine, Northwestern University, Chicago, Illinois.

${ }^{7}$ Department of Clinical Pharmacy, University of Colorado, Anschultz Medical Campus, and Clinical Specialist, Critical Care/Infectious Diseases, Department of Pharmacy Services, University of Colorado Hospital, Aurora, Colorado.

${ }^{8}$ Acute Care Surgery, Trauma, Burn, Critical Care, Emergency Surgery, and Associate Chair of Surgery, Critical Care, Department of Surgery, and Director, Surgical Critical Care, University of Michigan Health System, Ann Arbor, Michigan.

${ }^{9}$ Public Health Sciences, and Chief, Division of Acute Care Surgery and Outcomes Research, University of Virginia Health System, Charlottesville, Virginia.

${ }^{10}$ Pharmacy and Medicine, West Virginia University, Morgantown, West Virginia.

${ }^{11}$ Division of Infectious Diseases, Emory University, Atlanta, Georgia.

${ }^{12}$ Rush Medical College, Chicago, and Chairman, Department of Medicine, Cook County Health and Hospital System, Chicago, Illinois. Copyright (c) 2013, American Society of Health-System Pharmacists, Inc. All rights reserved. 1079-2082/13/0201-0195\$06.00.
} 
Gynecologists [ACOG]) and noted general guidelines (e.g., U.S. Centers for Disease Control and Prevention [CDC], Scottish Intercollegiate Guidelines Network, Medical Letter, SIS, SHEA/ IDSA) were also considered [2,3,5-11].

Recommendations for the use of antimicrobial prophylaxis are graded according to the strength of evidence available. The strength of evidence represents only support for or against prophylaxis and does not apply to the antimicrobial agent, dose, or dosage regimen. Studies supporting the recommendations for the use of antimicrobial therapy were classified as follows:

- Level I (evidence from large, well-conducted, randomized, controlled clinical trials or a meta-analysis),

- Level II (evidence from small, well-conducted, randomized, controlled clinical trials),

- Level III (evidence from well-conducted cohort studies),

- Level IV (evidence from well-conducted case-control studies),

- Level V (evidence from uncontrolled studies that were not well conducted),

- Level VI (conflicting evidence that tends to favor the recommendation), or

- Level VII (expert opinion or data extrapolated from evidence for general principles and other procedures).

This system has been used by the Agency for Healthcare Research and Quality, and ASHP, IDSA, SIS, and SHEA support it as an acceptable method for organizing strength of evidence for a variety of therapeutic or diagnostic recommendations [4]. Each recommendation was categorized according to the strength of evidence that supports the use or nonuse of antimicrobial prophylaxis as category A (levels I-III), category B (levels IV-VI), or category C (level VII).

When higher-level data are not available, a category $C$ recommendation represents a consensus of expert panel members based on their clinical experience, extrapolation from other procedures with similar microbial or other clinical features, and available published literature. In these cases, the expert panel also extrapolated general principles and evidence from other procedures. Some recommendations include alternative approaches in situations in which panel member opinions were divided.

A major limitation of the available literature on antimicrobial prophylaxis is the difficulty in establishing significant differences in efficacy between prophylactic antimicrobial agents and controls (including placebo, no treatment, or other antimicrobial agents) due to study design and low SSI rates for most procedures. A small sample size increases the likelihood of a Type II error; therefore, there may be no apparent difference between the antimicrobial agent and placebo when in fact the antimicrobial has a beneficial effect [12]. A valid study is placebo-controlled and randomized with a sufficient sample in each group to avoid a Type II error. Of note, prophylaxis is recommended in some cases due to the severity of complications of postoperative infection (e.g., an infected device that is not easily removable) necessitating precautionary measures despite the lack of statistical support.

\section{Summary of key updates}

These guidelines reflect substantial changes from the guidelines published in 1999 [1]. Highlights of those changes are outlined here.
Preoperative-dose timing. The optimal time for administration of preoperative doses is within $60 \mathrm{~min}$ before surgical incision. This is a more-specific time frame than the previously recommended time, which was "at induction of anesthesia." Some agents, such as fluoroquinolones and vancomycin, require administration over one to two hours; therefore, the administration of these agents should begin within $120 \mathrm{~min}$ before surgical incision.

Selection and dosing. Information is included regarding the approach to weight-based dosing in obese patients and the need for repeat doses during prolonged procedures [13-18]. Obesity has been linked to an increased risk for SSI. The pharmacokinetics of drugs may be altered in obese patients, so dosage adjustments based on body weight may be warranted in these patients. For all patients, intraoperative redosing is needed to ensure adequate serum and tissue concentrations of the antimicrobial if the duration of the procedure exceeds two half-lives of the drug or there is excessive blood loss during the procedure (Table 1). Recommendations for selection of antimicrobial agents for specific surgical procedures and alternative agents (e.g., for patients with allergies to $\beta$-lactam antimicrobials) are provided in Table 2.

Duration of prophylaxis. New recommendations for a shortened postoperative course of antimicrobials involving a single dose or continuation for less than 24 hours are provided. Further clarity on the lack of need for postoperative antimicrobial prophylaxis based on the presence of indwelling drains and intravascular catheters is included.

Common principles. A section addressing concepts that apply to all types of surgical procedures has been added. Expanded and new recommendations are provided for plastic, urology, cardiac, and thoracic procedures, as well as clarity on prophylaxis when implantable devices are inserted. The latest information on the use of mupirocin and on the role of vancomycin in surgical prophylaxis is summarized in these updated guidelines.

\section{Application of guidelines to clinical practice}

Recommendations are provided for adult (age 19 years or older) and pediatric (age 1-18 years) patients. These guidelines do not specifically address newborn (premature and fullterm) infants. While the guidelines do not address all concerns for patients with renal or hepatic dysfunction, antimicrobial prophylaxis often does not need to be modified for these patients when given as a single preoperative dose before surgical incision.

The recommendations herein may not be appropriate for use in all clinical situations. Decisions to follow these recommendations must be based on the judgment of the clinician and consideration of individual patient circumstances and available resources.

These guidelines reflect current knowledge of antimicrobial prophylaxis in surgery. Given the dynamic nature of scientific information and technology, periodic review, updating, and revisions are to be expected.

\section{Special patient populations}

Pediatric patients. Pediatric patients undergo a number of procedures similar to adults that may warrant 
Table 1. Recommended Doses and Redosing Intervals for Commonly Used Antimicrobials for Surgical Prophylaxis

\begin{tabular}{|c|c|c|c|c|}
\hline \multirow[b]{2}{*}{ Antimicrobial } & \multicolumn{2}{|c|}{ Recommended Dose } & \multirow{2}{*}{$\begin{array}{l}\text { Half-life in Adults } \\
\text { With Normal Renal } \\
\text { Function, } h \text { [19] }\end{array}$} & \multirow{2}{*}{$\begin{array}{l}\text { Recommended Redosing } \\
\text { Interval (From Initiation } \\
\text { of Preoperative Dose), } h^{\mathrm{c}}\end{array}$} \\
\hline & Adults ${ }^{\mathrm{a}}$ & Pediatrics $^{\mathrm{b}}$ & & \\
\hline $\begin{array}{l}\text { Ampicillin- } \\
\text { sulbactam }\end{array}$ & $\begin{array}{l}3 \mathrm{~g} \text { (ampicillin } \\
2 \mathrm{~g} / \text { sulbactam } 1 \mathrm{~g})\end{array}$ & $\begin{array}{l}50 \mathrm{mg} / \mathrm{kg} \text { of the } \\
\text { ampicillin } \\
\text { component }\end{array}$ & $0.8-1.3$ & 2 \\
\hline Ampicillin & $2 \mathrm{~g}$ & $50 \mathrm{mg} / \mathrm{kg}$ & $1-1.9$ & 2 \\
\hline Aztreonam & $2 \mathrm{~g}$ & $30 \mathrm{mg} / \mathrm{kg}$ & $1.3-2.4$ & 4 \\
\hline Cefazolin & $\begin{array}{l}2 \mathrm{~g}, 3 \mathrm{~g} \text { for pts } \\
\quad \text { weighing } \geq 120 \mathrm{~kg}\end{array}$ & $30 \mathrm{mg} / \mathrm{kg}$ & $1.2-2.2$ & 4 \\
\hline Cefuroxime & $1.5 \mathrm{~g}$ & $50 \mathrm{mg} / \mathrm{kg}$ & $1-2$ & 4 \\
\hline Cefotaxime & $1 \mathrm{gd}$ & $50 \mathrm{mg} / \mathrm{kg}$ & $0.9-1.7$ & 3 \\
\hline Cefoxitin & $2 \mathrm{~g}$ & $40 \mathrm{mg} / \mathrm{kg}$ & $0.7-1.1$ & 2 \\
\hline Cefotetan & $2 \mathrm{~g}$ & $40 \mathrm{mg} / \mathrm{kg}$ & $2.8-4.6$ & 6 \\
\hline Ceftriaxone & $2 \mathrm{~g}^{\mathrm{e}}$ & $50-75 \mathrm{mg} / \mathrm{kg}$ & $5.4-10.9$ & NA \\
\hline Ciprofloxacin ${ }^{f}$ & $400 \mathrm{mg}$ & $10 \mathrm{mg} / \mathrm{kg}$ & $3-7$ & NA \\
\hline Clindamycin & $900 \mathrm{mg}$ & $10 \mathrm{mg} / \mathrm{kg}$ & $2-4$ & 6 \\
\hline Ertapenem & $1 \mathrm{~g}$ & $15 \mathrm{mg} / \mathrm{kg}$ & $3-5$ & NA \\
\hline Fluconazole & $400 \mathrm{mg}$ & $6 \mathrm{mg} / \mathrm{kg}$ & 30 & NA \\
\hline Gentamicin $^{\mathrm{g}}$ & $\begin{array}{l}5 \mathrm{mg} / \mathrm{kg} \text { based on } \\
\text { dosing weight } \\
\text { (single dose) }\end{array}$ & $\begin{array}{l}2.5 \mathrm{mg} / \mathrm{kg} \text { based } \\
\text { on dosing weight }\end{array}$ & $2-3$ & NA \\
\hline Levofloxacin $^{\mathrm{f}}$ & $500 \mathrm{mg}$ & $10 \mathrm{mg} / \mathrm{kg}$ & $6-8$ & NA \\
\hline Metronidazole & $500 \mathrm{mg}$ & $\begin{array}{l}15 \mathrm{mg} / \mathrm{kg} \\
\text { Neonates weighing } \\
<1,200 \mathrm{~g} \text { should } \\
\text { receive a single } \\
7.5-\mathrm{mg} / \mathrm{kg} \text { dose }\end{array}$ & $6-8$ & NA \\
\hline Moxifloxacin ${ }^{\mathrm{f}}$ & $400 \mathrm{mg}$ & $10 \mathrm{mg} / \mathrm{kg}$ & 8-15 & NA \\
\hline $\begin{array}{l}\text { Piperacillin- } \\
\text { tazobactam }\end{array}$ & $3.375 \mathrm{~g}$ & $\begin{array}{l}\text { Infants } 2-9 \text { mo: } \\
80 \mathrm{mg} / \mathrm{kg} \text { of the } \\
\text { piperacillin } \\
\text { component } \\
\text { Children }>9 \mathrm{mo} \text { and } \\
\leq 40 \mathrm{~kg}: 100 \mathrm{mg} / \mathrm{kg} \\
\text { of the piperacillin } \\
\text { component }\end{array}$ & $0.7-1.2$ & 2 \\
\hline $\begin{array}{l}\text { Vancomycin } \\
\text { Oral antibiotics for } \\
\text { colorectal surgery } \\
\text { prophylaxis (used } \\
\text { in conjunction } \\
\text { with a mechanical } \\
\text { bowel preparation) }\end{array}$ & $15 \mathrm{mg} / \mathrm{kg}$ & $15 \mathrm{mg} / \mathrm{kg}$ & $4-8$ & NA \\
\hline Erythromycin base & $1 \mathrm{~g}$ & $20 \mathrm{mg} / \mathrm{kg}$ & $0.8-3$ & NA \\
\hline Metronidazole & $1 \mathrm{~g}$ & $15 \mathrm{mg} / \mathrm{kg}$ & $6-10$ & NA \\
\hline Neomycin & $1 \mathrm{~g}$ & $15 \mathrm{mg} / \mathrm{kg}$ & $\begin{array}{l}\text { 2-3 (3\% absorbed } \\
\text { under normal } \\
\text { gastrointestinal } \\
\text { conditions) }\end{array}$ & NA \\
\hline
\end{tabular}

\footnotetext{
${ }^{a}$ Adult doses are obtained from the studies cited in each section. When doses differed between studies, expert opinion used the most-often recommended dose.

${ }^{\mathrm{b}}$ The maximum pediatric dose should not exceed the usual adult dose.

${ }^{\text {c}}$ For antimicrobials with a short half-life (e.g., cefazolin, cefoxitin) used before long procedures, redosing in the operating room is recommended at an interval of approximately two times the half-life of the agent in patients with normal renal function. Recommended redosing intervals marked as "not applicable" (NA) are based on typical case length; for unusually long procedures, redosing may be needed.

${ }^{\mathrm{d}}$ Although FDA-approved package insert labeling indicates $1 \mathrm{~g},{ }^{14}$ experts recommend $2 \mathrm{~g}$ for obese patients.

eWhen used as a single dose in combination with metronidazole for colorectal procedures.

${ }^{\mathrm{f}}$ While fluoroquinolones have been associated with an increased risk of tendinitis/tendon rupture in all ages, use of these agents for singledose prophylaxis is generally safe.

${ }^{g}$ In general, gentamicin for surgical antibiotic prophylaxis should be limited to a single dose given preoperatively. Dosing is based on the patient's actual body weight. If the patient's actual weight is more than $20 \%$ above ideal body weight (IBW), the dosing weight (DW) can be determined as follows: DW=IBW +0.4(actual weight-IBW).
} 


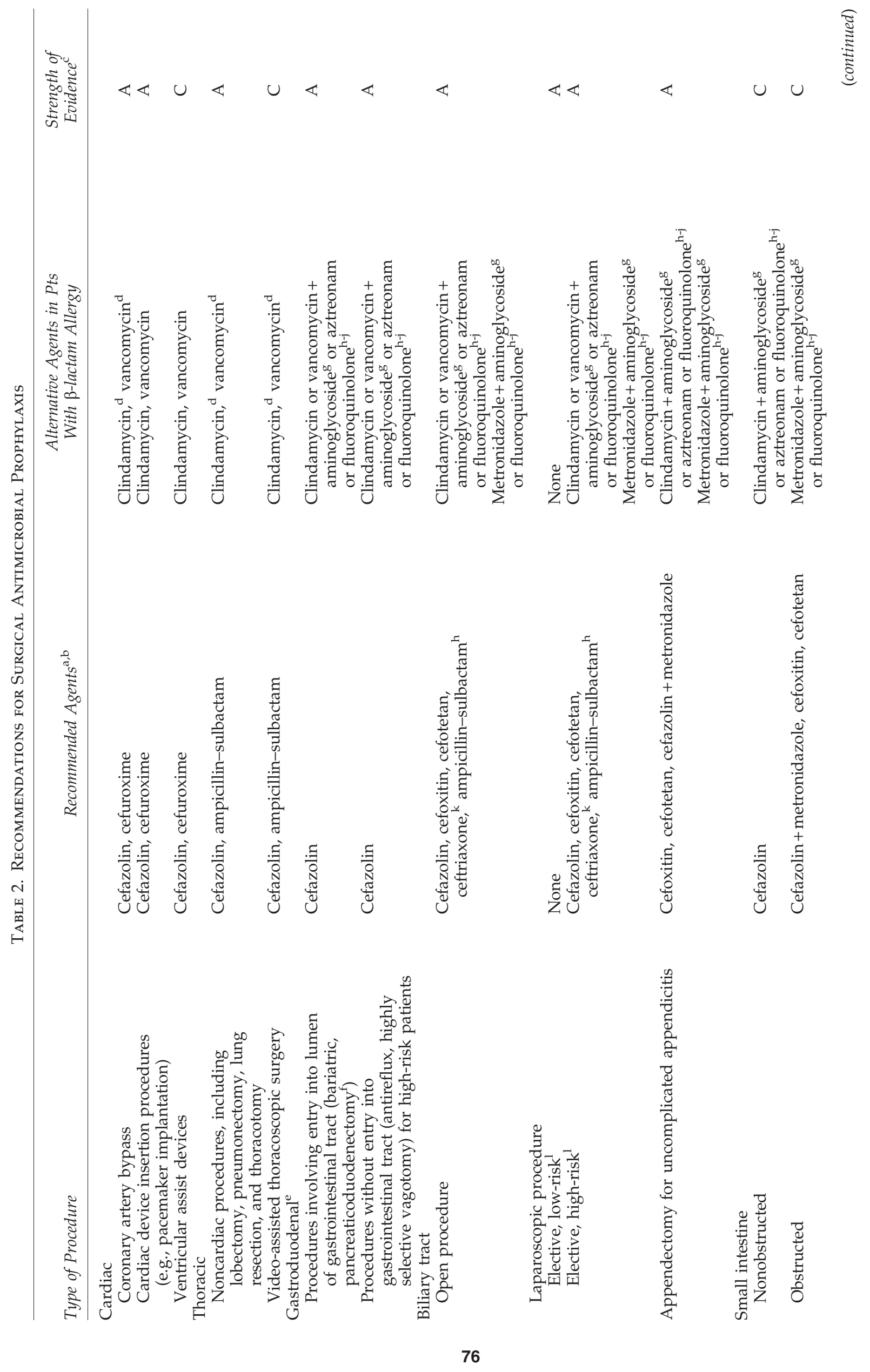




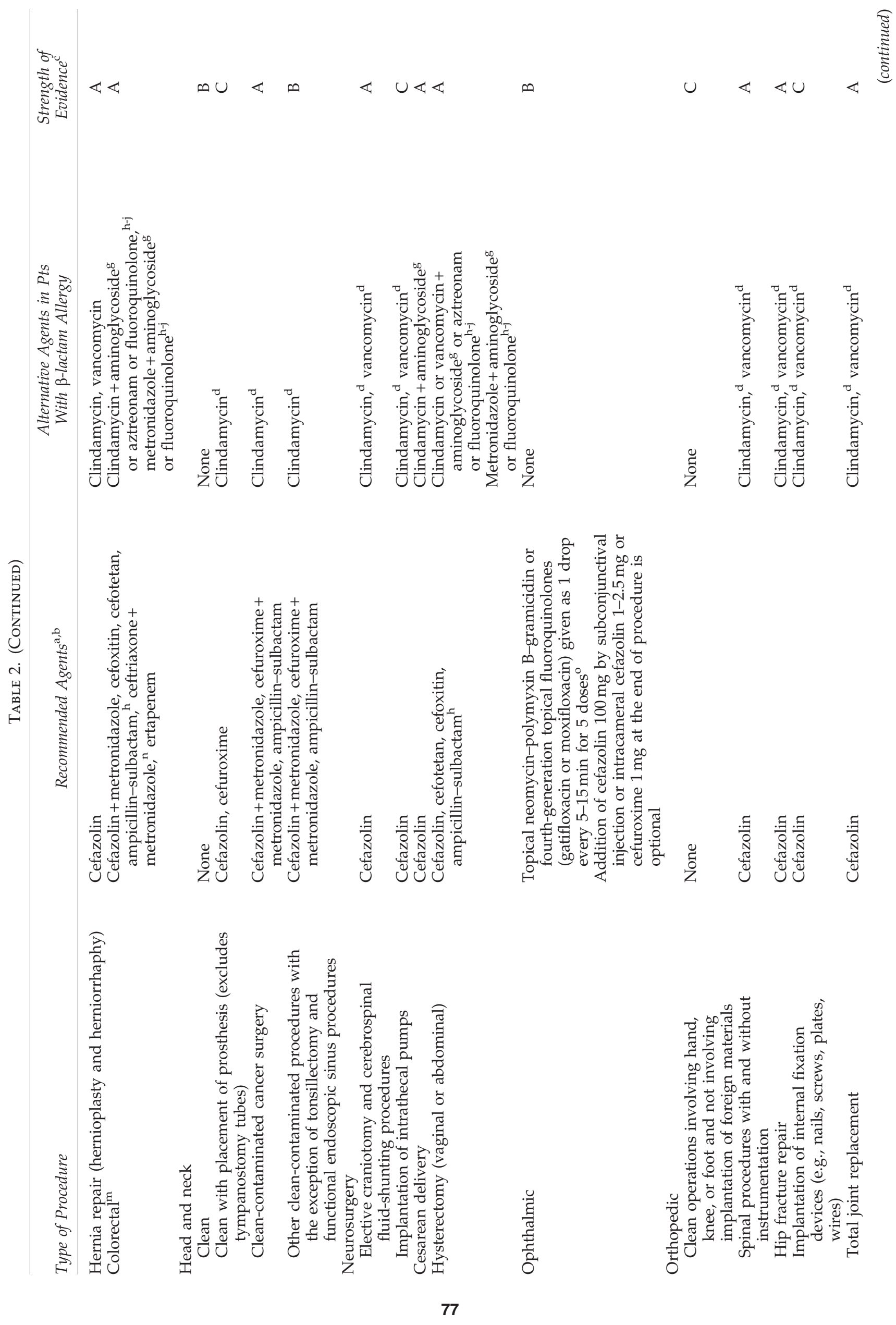




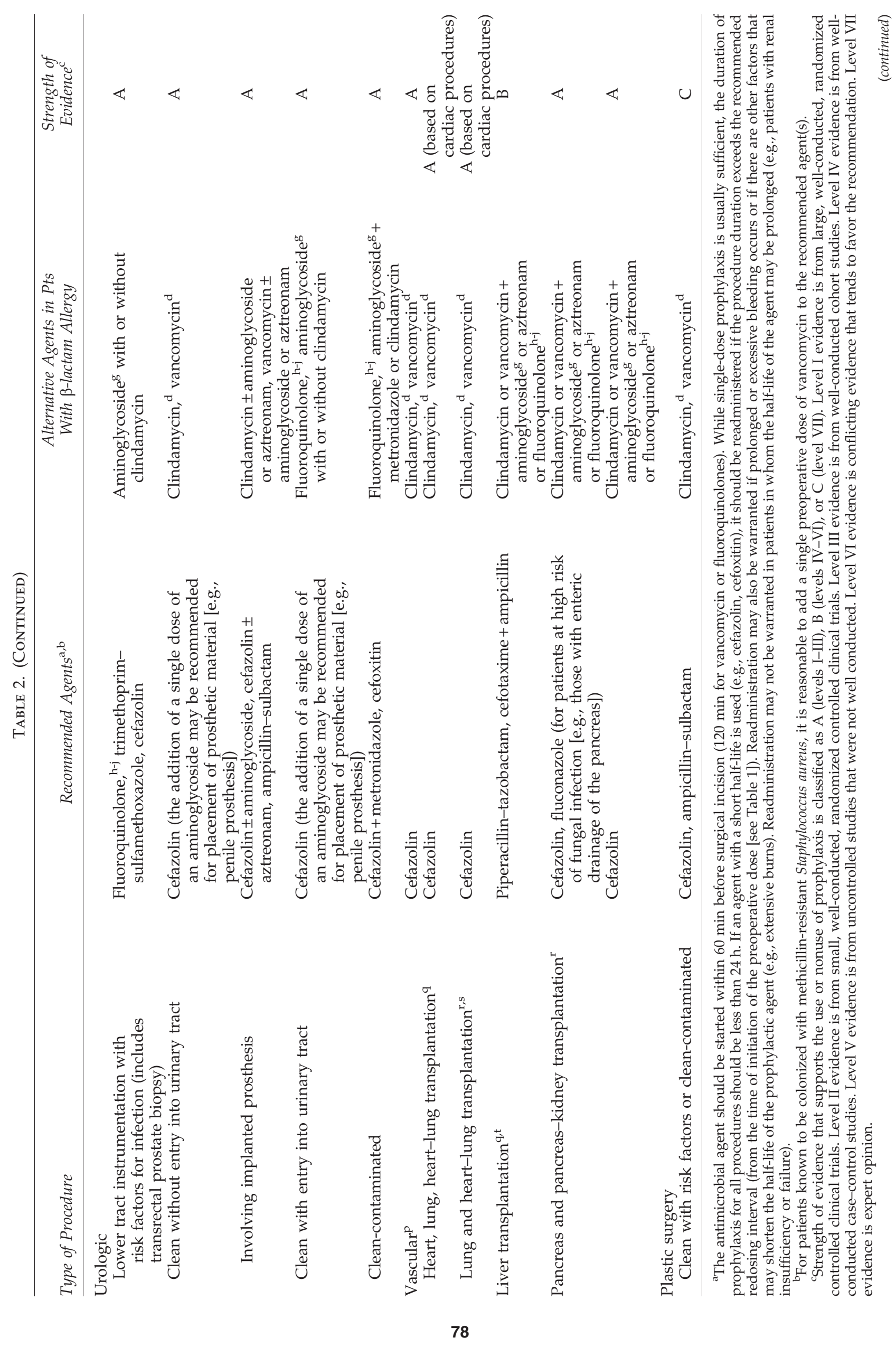




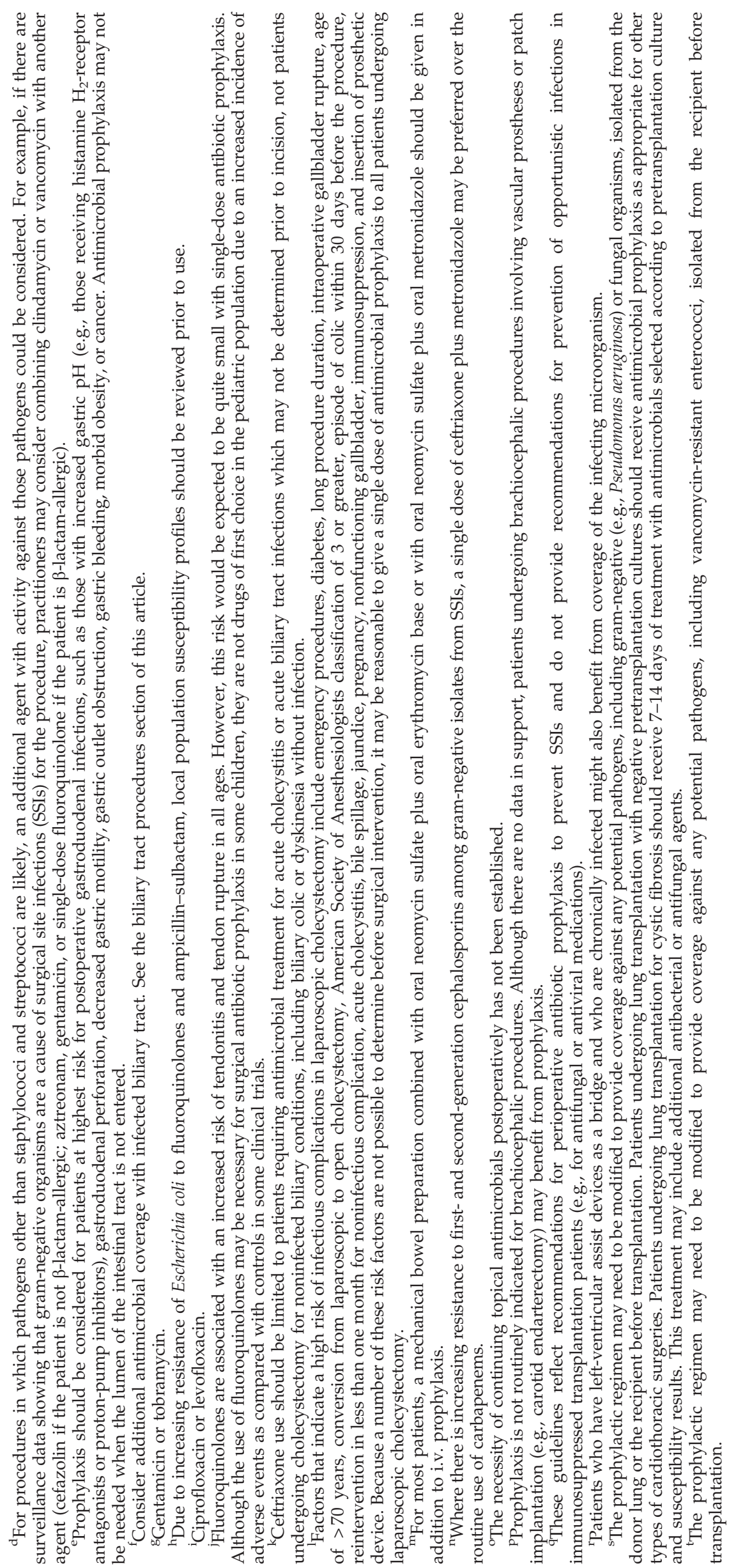


antimicrobial prophylaxis. Although pediatric-specific prophylaxis data are sparse, available data have been evaluated and are presented in some of the procedure-specific sections of these guidelines. Selection of antimicrobial prophylactic agents mirrors that in adult guidelines, with the agents of choice being first- and second-generation cephalosporins, reserving the use of vancomycin for patients with documented $\beta$-lactam allergies $[19,20]$. While the use of a penicillin with a $\beta$-lactamase inhibitor in combination with cefazolin or vancomycin and gentamicin has also been studied in pediatric patients, the number of patients included in these evaluations remains small [20-23]. As with adults, there is little evidence supporting the use of vancomycin, alone or in combination with other antimicrobials, for routine perioperative antimicrobial prophylaxis in institutions that have a high prevalence of methicillin-resistant Staphylococcus aureus (MRSA). Vancomycin may be considered in children known to be colonized with MRSA and, in one retrospective historical cohort study, was shown to decrease MRSA infections [21]. Mupirocin use has been studied in and is efficacious in children colonized with MRSA, but there are limited data supporting its use perioperatively [24-30]. However, there is little reason to think that the impact and effect would be any different in children, so its use may be justified. Additional studies in this setting are needed to establish firm guidelines.

Unless noted in specific sections, all recommendations for adults are the same for pediatric patients, except for dosing. In most cases, the data in pediatric patients are limited and have been extrapolated from adult data; therefore, nearly all pediatric recommendations are based on expert opinion. In some sections, pediatric efficacy data do not exist and thus are not addressed in these guidelines. Fluoroquinolones should not be routinely used for surgical prophylaxis in pediatric patients because of the potential for toxicity in this population. The same principle of preoperative dosing within $60 \mathrm{~min}$ before incision has been applied to pediatric patients [20-23]. Additional intraoperative dosing may be needed if the duration of the procedure exceeds two half-lives of the antimicrobial agent or there is excessive blood loss during the procedure $[19,21]$. As with adult patients, single-dose prophylaxis is usually sufficient. If antimicrobial prophylaxis is continued postoperatively, the duration should be less than $24 \mathrm{~h}$, regardless of the presence of intravascular catheters or indwelling drains $[19,22,23,31,32]$. There are sufficient pharmacokinetic studies of most agents to recommend pediatric dosages that provide adequate systemic exposure and, presumably, efficacy comparable to that demonstrated in adults. Therefore, the pediatric dosages provided in these guidelines are based largely on pharmacokinetic data and the extrapolation of adult efficacy data to pediatric patients. Because few clinical trials have been conducted in pediatric surgical patients, strength of evidence criteria have not been applied to these recommendations. With few exceptions (e.g., aminoglycoside dosages), pediatric dosages should not exceed the maximum adult recommended dosages. Generally, if dosages are calculated on a milligram-per-kilogram basis for children weighing more than $40 \mathrm{~kg}$, the calculated dosage will exceed the maximum recommended dosage for adults; adult dosages should therefore be used.

Patients with prosthetic implants. For patients with existing prosthetic implants who undergo an invasive proce- dure, there is no evidence that antimicrobial prophylaxis prevents infections of the implant. However, updated guidelines from the American Heart Association (AHA) suggest that prophylaxis may be justified in a limited subset of patients for the prevention of endocarditis [11].

\section{Common principles and procedure-specific guidelines}

The Common Principles section has been developed to provide information common to many surgical procedures. These principles are general recommendations based on currently available data at the time of publication that may change over time; therefore, these principles need to be applied with careful attention to each clinical situation. Detailed information pertinent to specific surgical procedures is included in the procedure-specific sections of these guidelines.

In addition to patient- and procedure-specific considerations, several institution-specific factors must be considered by practitioners before instituting these guidelines. The availability of antimicrobial agents at the institution may be restricted by local antimicrobial-use policy or lack of approval for use by regulatory authorities. Medications that are no longer available or not approved for use by the U.S. Food and Drug Administration (FDA) are so noted. Local resistance patterns should also be considered in selecting antimicrobial agents and are discussed in the colonization and resistance patterns section of the Common Principles section.

\section{Requirements for effective surgical prophylaxis}

Appendix A lists the wound classification criteria currently used by the CDC National Healthcare Safety Network (NHSN) and Healthcare Infection Control Practices Advisory Committee (HICPAC) [33-35]. Criteria for defining an SSI have also been established by NHSN (Appendix B) $[8,36]$. These definitions assist in evaluating the importance of providing antimicrobial prophylaxis and the potential consequences of infection, including the need for treatment. Some criteria vary slightly by procedure.

Although antimicrobial prophylaxis plays an important role in reducing the rate of SSIs, other factors such as attention to basic infection-control strategies [37], the surgeon's experience and technique, the duration of the procedure, hospital and operating-room environments, instrument-sterilization issues, preoperative preparation (e.g., surgical scrub, skin antisepsis, appropriate hair removal), perioperative management (temperature and glycemic control), and the underlying medical condition of the patient may have a strong impact on SSI rates $[5,8]$. These guidelines recognize the importance of these other factors but do not include a discussion of or any recommendations regarding these issues beyond the optimal use of prophylactic antimicrobial agents. Patient-related factors associated with an increased risk of SSI include extremes of age, nutritional status, obesity, diabetes mellitus, tobacco use, coexistent remote body-site infections, altered immune response, corticosteroid therapy, recent surgical procedure, length of preoperative hospitalization, and colonization with microorganisms. Antimicrobial prophylaxis may be justified for any procedure if the patient has an underlying medical condition associated with a high risk of SSI or if the patient is immunocompromised (e.g., malnourished, neutropenic, receiving immunosuppressive agents). 
Antimicrobial prophylaxis may be beneficial in surgical procedures associated with a high rate of infection (i.e., cleancontaminated or contaminated procedures) and in certain clean procedures where there are severe consequences of infection (e.g., prosthetic implants), even if infection is unlikely. While prophylactic antimicrobials are not indicated for some clean surgical procedures [8], available data suggest that the relative risk reduction of SSI from the use of antimicrobial prophylaxis is the same in clean and in higher-risk procedures [38]. The decision to use prophylaxis depends on the cost of treating and the morbidity associated with infection compared with the cost and morbidity associated with using prophylaxis. Antimicrobial prophylaxis is justified for most clean-contaminated procedures. The use of antimicrobial agents for dirty procedures (Appendix A) or established infections is classified as treatment of presumed infection, not prophylaxis. See the procedure-specific sections for detailed recommendations.

\section{Quality improvement efforts}

National, state, local, and institutional groups have developed and implemented collaborative efforts to improve the appropriateness of surgical antimicrobial prophylaxis. Various process and outcomes measures are employed, and results are disseminated. Institutional epidemiology and infection-control programs, state-based quality-improvement campaigns (e.g., the Michigan Surgical Quality Collaborative, the Washington State Surgical Clinical Outcomes Assessment Program [39,40]), CDC, NHSN, the National Surgical Quality Improvement Program, The Joint Commission, and the National Quality Forum have been instrumental in developing programs to prevent SSIs.

Over the past decade or more, several organizations, payers, and government agencies, including the Centers for Medicare and Medicaid Services (CMS), have established national quality-improvement initiatives to further improve the safety and outcomes of health care, including surgery [41-47]. One area of focus in these initiatives for patients undergoing surgical procedures is the prevention of SSIs. The performance measures used, data collection and reporting requirements, and financial implications vary among the initiatives. The Surgical Care Improvement Project (SCIP) began in 2002 as the Surgical Infection Prevention (SIP) project, focusing on the timing, selection, and duration of prophylactic antimicrobial agents [41,42]. The SIP project was expanded to SCIP to include additional process measures surrounding patient safety and care during surgical procedures, including glucose control, venous thromboembolism prophylaxis, hair removal, and temperature control. Similar measures have been adopted by The Joint Commission [43]. The Physicians Quality Reporting System was established in 2006 to provide financial incentives to physicians meeting performance standards for quality measures, including surgery-related measures similar to those reported for SCIP and the Joint Commission [44]. Data are required to be collected by institutions and reported to payers [42,44,46]. Data for CMS and the Physicians Quality Reporting System measures are displayed on public websites to allow consumers to compare performance among hospitals. Institutional data collection and reporting are required, with financial incentives tied to performance to varying degrees, including payment for reporting, payment increases for meeting or exceeding minimum levels of performance, payment reduction for poor performance, and lack of payment for the development of surgical complications, such as mediastinitis.

Quality improvement initiatives and mandated performance reporting are subject to change, so readers of these guidelines are advised to consult their local or institutional quality-improvement departments for new developments in requirements for measures and data reporting that apply to their practice.

\section{Cost containment}

Few pharmacoeconomic studies have addresed surgical antimicrobial prophylaxis; therefore, a cost-minimization approach was employed in developing these guidelines. The antimicrobial agent recommendations are based primarily on efficacy and safety. Individual institutions must consider their acquisition costs when implementing these guidelines.

Additional cost savings may be realized through collaborative management by pharmacists and surgeons to select the most cost-effective agent and minimize or eliminate postoperative dosing [48-50]. The use of standardized antimicrobial order sets, automatic stop-order programs, and educational initiatives has been shown to facilitate the adoption of guidelines for surgical antimicrobial prophylaxis [51-58].

\section{Common principles}

Ideally, an antimicrobial agent for surgical prophylaxis should (1) prevent SSI, (2) prevent SSI-related morbidity and mortality, (3) reduce the duration and cost of health care (when the costs associated with the management of SSI are considered, the cost-effectiveness of prophylaxis becomes evident) [51,52], (4) produce no adverse effects, and (5) have no adverse consequences for the microbial flora of the patient or the hospital [53]. To achieve these goals, an antimicrobial agent should be (1) active against the pathogens most likely to contaminate the surgical site, (2) given in an appropriate dosage and at a time that ensures adequate serum and tissue concentrations during the period of potential contamination, (3) safe, and (4) administered for the shortest effective period to minimize adverse effects, the development of resistance, and costs $[8,59,60]$.

The selection of an appropriate antimicrobial agent for a specific patient should take into account the characteristics of the ideal agent, the comparative efficacy of the antimicrobial agent for the procedure, the safety profile, and the patient's medication allergies. A full discussion of the safety profile, including adverse events, drug interactions, contraindications, and warnings, for each antimicrobial agent is beyond the scope of these guidelines. Readers of these guidelines should review the FDA-approved prescribing information and published data for specific antimicrobial agents before use. For most procedures, cefazolin is the drug of choice for prophylaxis because it is the most widely studied antimicrobial agent, with proven efficacy. It has a desirable duration of action, spectrum of activity against organisms commonly encountered in surgery, reasonable safety, and low cost. There is little evidence to suggest that broad-spectrum antimicrobial agents (i.e., agents with broad in vitro antibacterial activity) result in lower rates of postoperative SSI compared with older antimicrobial agents with a narrower spectrum of activity. However, comparative studies are limited by small sample sizes, resulting in difficulty detecting a significant difference between antimicrobial agents; therefore, antimicrobial selection is based 
on cost, safety profile, ease of administration, pharmacokinetic profile, and bactericidal activity.

\section{Common surgical pathogens}

The agent chosen should have activity against the most common surgical site pathogens. The predominant organisms causing SSIs after clean procedures are skin flora, including S. aureus and coagulase-negative staphylococci (e.g., S. epidermidis) [61]. In clean-contaminated procedures, including abdominal procedures and heart, kidney, and liver transplantations, the predominant organisms include gramnegative rods and enterococci in addition to skin flora. Additional details on common organisms can be found in procedure-specific sections of these guidelines.

Recommendations for the selection of prophylactic antimicrobials for various surgical procedures are provided in Table 2. Adult and pediatric dosages are included in Table 1. Agents that are FDA-approved for use in surgical antimicrobial prophylaxis include cefazolin, cefuroxime, cefoxitin, cefotetan, ertapenem, and vancomycin [62-67].

\section{Trends in microbiology}

The causative pathogens associated with SSIs in U.S. hospitals have changed over the past two decades. Analysis of National Nosocomial Infections Surveillance (NNIS) System data found that the percentage of SSIs caused by gramnegative bacilli decreased from $56.5 \%$ in 1986 to $33.8 \%$ in 2003 [68]. Staphylococcus aureus was the most common pathogen, causing $22.5 \%$ of SSIs during this time period. NHSN data from 2006 to 2007 revealed that the proportion of SSIs caused by $S$. aureus increased to $30 \%$, with MRSA comprising $49.2 \%$ of these isolates [61]. In a study of patients readmitted to U.S. hospitals between 2003 and 2007 with a culture-confirmed SSI, the proportion of infections caused by MRSA increased significantly from $16.1 \%$ to $20.6 \%(p<0.0001)$ [69]. The MRSA infections were associated with higher mortality rates, longer hospital stays, and higher hospital costs compared with other infections.

\section{Spectrum of activity}

Antimicrobial agents with the narrowest spectrum of activity required for efficacy in preventing infection are recommended in these guidelines. Alternative antimicrobial agents with documented efficacy are also listed herein. Individual health systems must consider local resistance patterns of organisms and overall SSI rates at their site when adopting these recommendations. Resistance patterns from organisms causing SSIs-in some cases procedure-specific resistance patterns-should take precedence over hospitalwide antibiograms.

\section{Vancomycin}

In 1999, HICPAC, an advisory committee to CDC and the Secretary of the Department of Health and Human Services, collaborated with other major organizations to develop recommendations for preventing and controlling vancomycin resistance [70]. The recommendations are echoed by these and other guidelines $[6,7,41,71]$. Routine use of vancomycin prophylaxis is not recommended for any procedure [8]. Vancomycin may be included in the regimen of choice when a cluster of MRSA cases (e.g., mediastinitis after cardiac procedures) or methicillin-resistant coagulase-negative staphylococci SSIs have been detected at an institution. Vancomycin prophylaxis should be considered for patients with known MRSA colonization or at high risk for MRSA colonization in the absence of surveillance data (e.g., patients with recent hospitalization, nursing-home residents, hemodialysis patients) [5,41,72]. In institutions with SSIs attributable to community-associated MRSA, antimicrobial agents with known in vitro activity against this pathogen may be considered as an alternative to vancomycin.

Each institution is encouraged to develop guidelines for the proper use of vancomycin. Although vancomycin is commonly used when the risk for MRSA is high, data suggest that vancomycin is less effective than cefazolin for preventing SSIs caused by methicillin-susceptible $S$. aureus (MSSA) [73,74]. For this reason, vancomycin is used in combination with cefazolin at some institutions with both MSSA and MRSA SSIs. For procedures in which pathogens other than staphylococci and streptococci are likely, an additional agent with activity against those pathogens should be considered. For example, if there are surveillance data showing that gram-negative organisms are a cause of SSIs for the procedure, practitioners may consider combining vancomycin with another agent (cefazolin if the patient does not have a $\beta$-lactam allergy; an aminoglycoside [gentamicin or tobramycin], aztreonam, or single-dose fluoroquinolone if the patient has a $\beta$-lactam allergy). The use of vancomycin for MRSA prophylaxis does not supplant the need for routine surgical prophylaxis appropriate for the type of procedure. When vancomycin is used, it can almost always be used as a single dose due to its long half-life.

\section{Colonization and resistance}

A national survey determined that $S$. aureus nasal colonization in the general population decreased from $32.4 \%$ in $2001-02$ to $28.6 \%$ in $2003-04$ ( $\mathrm{p}<0.01$ ), whereas the prevalence of colonization with MRSA increased from $0.8 \%$ to $1.5 \%$ $(p<0.05)$ during the same time periods [75]. Colonization with MRSA was independently associated with health care exposure among men, having been born in the United States, age of $>60$ years, diabetes, and poverty among women. Similarly, children are colonized with $S$. aureus and MRSA, but colonization varies by age. Children under five years of age have the highest rates, mirroring rates seen in patients over age 60 years [76]. The rates drop in children between five and 14 years of age and gradually increase to rates seen in the adult population. Lo et al. [77]. reported that in a large cohort of children, 28.1\% were colonized with S. aureus between 2004 and 2006. Between 2007 and 2009, 23.3\% of children were colonized with $S$. aureus, but the proportion of children colonized with MRSA had increased from $8.1 \%$ in 2004 to $15.1 \%$ in 2009.

Surgical antimicrobial prophylaxis can alter individual and institutional bacterial flora, leading to changes in colonization rates and increased bacterial resistance [78-84]. Surgical prophylaxis can also predispose patients to Clostridium difficile-associated colitis [81]. Risk factors for development of C. difficile-associated colitis include longer duration of prophylaxis or therapy and use of multiple antimicrobial agents [85]. Limiting the duration of antimicrobial prophylaxis to a single preoperative dose can reduce the risk of $C$. difficile disease. 
The question of what antimicrobial surgical prophylaxis to use for patients known to be colonized or recently infected with multidrug-resistant pathogens cannot be answered easily or in a manner that can be applied uniformly to all patient scenarios. Whether prophylaxis should be expanded to provide coverage for these pathogens depends on many factors, including the pathogen, its antimicrobial susceptibility profile, the host, the procedure to be performed, and the proximity of the likely reservoir of the pathogen to the incision and operative sites. While there is no evidence on the management of surgical antimicrobial prophylaxis in a patient with past infection or colonization with a resistant gram-negative pathogen, it is logical to provide prophylaxis with an agent active against MRSA for any patient known to be colonized with this gram-positive pathogen who will have a skin incision; specific prophylaxis for a resistant gram-negative pathogen in a patient with past infection or colonization with such a pathogen may not be necessary for a purely cutaneous procedure. Similarly, a patient colonized with vancomycin-resistant enterococci (VRE) should receive prophylaxis effective against VRE when undergoing liver transplantation but probably not when undergoing an umbilical hernia repair without mesh placement. Thus, patients must be treated on a case-by-case basis, taking into account multiple considerations.

Patients receiving therapeutic antimicrobials for a remote infection before surgery should also be given antimicrobial prophylaxis before surgery to ensure adequate serum and tissue levels of antimicrobials with activity against likely pathogens for the duration of the operation. If the agents used therapeutically are appropriate for surgical prophylaxis, administering an extra dose within 60 min before surgical incision is sufficient. Otherwise, the antimicrobial prophylaxis recommended for the planned procedure should be used. For patients with indwelling tubes or drains, consideration may be given to using prophylactic agents active against pathogens found in these devices before the procedure, even though therapeutic treatment for pathogens in drains is not indicated at other times. For patients with chronic renal failure receiving vancomycin, a preoperative dose of cefazolin should be considered instead of an extra dose of vancomycin, particularly if the probable pathogens associated with the procedure are gram-negative. In most circumstances, elective surgery should be postponed when the patient has an infection at a remote site.

\section{Allergy to $\beta$-lactam antimicrobials}

Allergy to $\beta$-lactam antimicrobials may be a consideration in the selection of surgical prophylaxis. The $\beta$-lactam antimicrobials, including cephalosporins, are the mainstay of surgical antimicrobial prophylaxis and are also the most commonly implicated drugs when allergic reactions occur. Because the predominant organisms in SSIs after clean procedures are gram-positive, the inclusion of vancomycin may be appropriate for a patient with a life-threatening allergy to $\beta$-lactam antimicrobials.

Although true Type 1 (immunoglobulin E [IgE]-mediated) cross-allergic reactions between penicillins, cephalosporins, and carbapenems are uncommon, cephalosporins and carbapenems should not be used for surgical prophylaxis in patients with documented or presumed IgE-mediated penicillin allergy. Confusion about the definition of true allergy among patients and practitioners leads to recommendations for alternative antimicrobial therapy with the potential for a lack of efficacy, increased cost, and adverse events [86,87]. Type 1 anaphylactic reactions to antimicrobials usually occur 30-60 min after administration. In patients receiving penicillins, this reaction is a life-threatening emergency that precludes subsequent use of penicillins [88]. Cephalosporins and carbapenems can safely be used in patients with an allergic reaction to penicillins that is not an IgE-mediated reaction (e.g., anaphylaxis, urticaria, bronchospasm) or exfoliative dermatitis (Stevens-Johnson syndrome, toxic epidermal necrolysis), a life-threatening hypersensitivity reaction that can be caused by $\beta$-lactam antimicrobials and other medications $[88,89]$. Patients should be carefully questioned about their history of antimicrobial allergies to determine whether a true allergy exists before selection of agents for prophylaxis. Patients with allergies to cephalosporins, penicillins, or both have been excluded from many clinical trials. Alternatives to $\beta$-lactam antimicrobials are provided in Table 2 based mainly on the antimicrobial activity profiles against predominant procedure-specific organisms and available clinical data.

\section{Drug administration}

The preferred route of administration varies with the type of procedure, but for a majority of procedures, i.v. administration is ideal because it produces rapid, reliable, and predictable serum and tissue concentrations.

\section{Timing of initial dose}

Successful prophylaxis requires the delivery of the antimicrobial to the operative site before contamination occurs. Thus, the antimicrobial agent should be administered at such a time to provide serum and tissue concentrations exceeding the minimum inhibitory concentration (MIC) for the probable organisms associated with the procedure, at the time of incision, and for the duration of the procedure [41,90]. In 1985, DiPiro et al [91]. demonstrated that higher serum and tissue cephalosporin concentrations at the time of surgical incision and at the end of the procedure were achieved when the drugs were given intravenously at the time of anesthesia induction compared with administration in the operating room. The average interval between antimicrobial administration and incision was 17-22 minutes [91] (Dellinger EP, personal communication, May, 2011).

A prospective evaluation of 1,708 surgical patients receiving antimicrobial prophylaxis found that preoperative administration of antimicrobials within $2 \mathrm{~h}$ before surgical incision decreased the risk of SSI to $0.59 \%$, compared with $3.8 \%$ for early administration (2-24 h before surgical incision) and $3.3 \%$ for any postoperative administration (any time after incision) [92]. In a study of 2,048 patients undergoing coronary bypass graft or valve replacement surgery receiving vancomycin prophylaxis, the rate of SSI was lowest in those patients in whom an infusion was started 16-60 min before surgical incision [93]. This time interval (16-60 min before incision) was compared with four others, and the rates of SSIs were significantly lower when compared with infusions given $0-15$ min before surgical incision $(\mathrm{p}<0.01)$ and $121-180 \mathrm{~min}-$ utes before incision $(\mathrm{p}=0.037)$. The risk of infection was higher in patients receiving infusions 61-120 min before incision (odds ratio [OR], 2.3; 95\% confidence interval [CI], 0.98-5.61) and for patients whose infusions were started more 
than $180 \mathrm{~min}$ before surgical incision (OR, 2.1; 95\% CI, $0.82-5.62)[93]$.

In a large, prospective, multicenter study from the Trial to Reduce Antimicrobial Prophylaxis Errors (TRAPE) study group, the timing, duration, and intraoperative redosing of antimicrobial prophylaxis and risk of SSI were evaluated in 4,472 patients undergoing cardiac surgery, hysterectomy, or hip or knee arthroplasty [94]. The majority of patients (90\%) received antimicrobial prophylaxis per the SCIP guidelines [41]. Patients were assigned to one of four groups for analysis. Group $1(n=1,844)$ received a cephalosporin (or other antimicrobial with a short infusion time) administered within 30 min before incision or vancomycin or a fluoroquinolone within one hour before incision. Group $2(n=1796)$ received a cephalosporin 31-60 min before incision or vancomycin 61$120 \mathrm{~min}$ before incision. Group $3(n=644)$ was given antimicrobials earlier than recommended, and group $4(n=188)$ received their initial antimicrobial doses after incision. The infection risk was lowest in group 1 (2.1\%), followed by group $2(2.4 \%)$ and group $3(2.8 \%)$. The risk of infection was highest in group $4(5.3 \%, p=0.02$ compared with group 1$)$. When cephalosporins and other antimicrobials with short infusion times were analyzed separately $(n=3,656)$, the infection rate with antimicrobials administered within $30 \mathrm{~min}$ before incision was $1.6 \%$ compared with $2.4 \%$ when antimicrobials were administered 31-60 $\mathrm{min}$ before incision $(\mathrm{p}=0.13)$.

In a multicenter Dutch study of 1,922 patients undergoing total hip arthroplasty, the lowest SSI rate was seen in patients who received the antimicrobial during the $30 \mathrm{~min}$ before incision [95]. The highest risk for infection was found in patients who received prophylaxis after the incision.

It seems intuitive that the entire antimicrobial dose should be infused before a tourniquet is inflated or before any other procedure that restricts blood flow to the surgical site is initiated; however, a study of total knee arthroplasties compared cefuroxime given 10-30 min before tourniquet inflation with cefuroxime given $10 \mathrm{~min}$ before tourniquet deflation and found no significant difference in SSI rates between the two groups [96].

Overall, administration of the first dose of antimicrobial beginning within $60 \mathrm{~min}$ before surgical incision is recommended $[41,94,97]$. Administration of vancomycin and fluoroquinolones should begin within 120 minutes before surgical incision because of the prolonged infusion times required for these drugs. Because these drugs have long halflives, this early administration should not compromise serum levels of these agents during most surgical procedures. Although the recent data summarized above suggest lower infection risk with antimicrobial administration beginning within $30 \mathrm{~min}$ before surgical incision, these data are not sufficiently robust to recommend narrowing the optimal window to begin infusion to 1-30 min before surgical incision. However, these data do suggest that antimicrobials can be administered too close to the time of incision. Although a few articles have suggested increased infection risk with administration too close to the time of incision $[93,96,97]$, the data presented are not convincing. In fact, all of these articles confirm the increased rate of SSI for antimicrobials given earlier than $60 \mathrm{~min}$ before incision. In one article, the infection rate for patients given an antimicrobial within $15 \mathrm{~min}$ of incision was lower than when antimicrobials were given 15-30 $\mathrm{min}$ before incision [97]. In another article, small numbers of patients were reported, and an assertion of high infection rates for infusion within $15 \mathrm{~min}$ of incision was made, but no numeric data or $p$ values were provided [98]. In a third article, only 15 of over 2,000 patients received antimicrobials within 15 minutes before incision [93]. Earlier studies found that giving antimicrobials within $20 \mathrm{~min}$ of incision and as close as $7 \mathrm{~min}$ before incision resulted in therapeutic levels in tissue at the time of incision [41,90,91,94,97,98].

\section{Dosing}

To ensure that adequate serum and tissue concentrations of antimicrobial agents for prophylaxis of SSIs are achieved, antimicrobial-specific pharmacokinetic and pharmacodynamic properties and patient factors must be considered when selecting a dose. One of the earliest controlled studies of antimicrobial prophylaxis in cardiac surgery found a lower rate of infection in patients with detectable concentrations of the drug in serum at the end of surgery compared with patients in whom the drug was undetectable [99]. In another study, higher levels of antimicrobial in atrial tissue at the time of starting the pump for open-heart surgery were associated with fewer infections than were lower antimicrobial concentrations [100]. In patients undergoing colectomy, infection levels were inversely related to the serum gentamicin concentration at the time of surgical closure [17]. In general, it seems advisable to administer prophylactic agents in a manner that will ensure adequate levels of drug in serum and tissue for the interval during which the surgical site is open.

Weight-based dosing. The dosing of most antimicrobials in pediatric patients is based on body weight, but the dosing of many antimicrobials in adults is not based on body weight, because it is safe, effective, and convenient to use standardized doses for most of the adult patient population. Such standardized doses avoid the need for calculations and reduce the risk for medication errors. However, in obese patients, especially those who are morbidly obese, serum and tissue concentrations of some drugs may differ from those in normal-weight patients because of pharmacokinetic alterations that depend on the lipophilicity of the drug and other factors [101]. Limited data are available on the optimal approach to dosing of antimicrobial agents for obese patients [102,103]. If weight-based dosing is warranted for obese patients, it has not been determined whether the patient's ideal body weight or total (i.e., actual) body weight should be used. In theory, using the ideal body weight as the basis for dosing a lipophilic drug (e.g., vancomycin) could result in subtherapeutic concentrations in serum and tissue, and the use of actual body weight for dosing a hydrophilic drug (e.g., an aminoglycoside) could result in excessive concentrations in serum and tissue. Pediatric patients weighing more than $40 \mathrm{~kg}$ should receive weight-based doses unless the dose or daily dose exceeds the recommended adult dose [104].

Conclusive recommendations for weight-based dosing for antimicrobial prophylaxis in obese patients cannot be made because data demonstrating clinically relevant decreases in SSI rates from the use of such dosing strategies instead of standard doses in obese patients are not available in the published literature.

In a small, nonrandomized, two-phase study of morbidly obese adults undergoing gastroplasty and normal-weight 
adults undergoing upper abdominal surgery, blood and tissue concentrations of cefazolin after the administration of a $1 \mathrm{~g}$ preoperative dose were consistently lower in morbidly obese patients than in the normal-weight patients [101]. The concentrations in morbidly obese patients also were lower than the MICs needed for prophylaxis against gram-positive cocci and gram-negative rods. In the second phase of the study, adequate blood and tissue cefazolin concentrations were achieved in morbidly obese patients receiving preoperative doses of cefazolin $2 \mathrm{~g}$, and the rate of SSIs was significantly lower in these patients compared with morbidly obese patients receiving $1 \mathrm{~g}$ doses during the first phase of the study.

While the optimal cefazolin dose has not been established in obese patients, a few pharmacokinetic studies have investigated the cefazolin concentrations in serum and tissue during surgical procedures $[13,105]$. Two small pharmacokinetic studies found that administering 1- or $2 \mathrm{~g}$ doses of cefazolin may not be sufficient to produce serum and tissue concentrations exceeding the MIC for the most common pathogens. In a small, single-center study, 38 adults undergoing Rouxen-Y gastric bypass surgery were classified by body mass index (BMI) in one of three groups [13]. All patients were given cefazolin $2 \mathrm{~g}$ i.v. 30-60 $\mathrm{min}$ before the incision, followed by a second 2-g i.v. dose $3 \mathrm{~h}$ later. The mean serum drug concentration before the second dose of cefazolin was lower than the resistance breakpoint in all three BMI groups. Serum drug concentrations were lower in patients with a high BMI than in patients with lower BMI values. Tissue drug concentrations were lower than a targeted concentration of $8 \mathrm{mg} / \mathrm{mL}$ at all measurement times, except the time of skin closure in the patients with the lowest BMIs. These results suggest that a 1-g dose of cefazolin may be inadequate for obese patients undergoing gastric bypass surgery. A weakness of the literature on drug dosing in morbidly obese patients is the practice of reporting results by BMI rather than weight.

Doubling the normal dose of cephalosporins or making fewer adjustments based on renal dysfunction may produce concentrations in obese patients similar to those achieved with standard doses in normal-weight patients [103]. Considering the low cost and favorable safety profile of cefazolin, increasing the dose to $2 \mathrm{~g}$ for patients weighing more than 80 $\mathrm{kg}$ and to $3 \mathrm{~g}$ for those weighing over $120 \mathrm{~kg}$ can easily be justified [41]. For simplification, some hospitals have standardized $2 \mathrm{~g}$ cefazolin doses for all adult patients.

Gentamicin doses have been compared for prophylaxis only in colorectal surgery, where a single dose of gentamicin $4.5 \mathrm{mg} / \mathrm{kg}$ in combination with metronidazole was more effective in SSI prevention than multiple doses of gentamicin $1.5 \mathrm{mg} / \mathrm{kg}$ every eight hours $[16,17]$. In obese patients who weigh $20 \%$ above their ideal body weight, the dose of gentamicin should be calculated using the ideal body weight plus $40 \%$ of the difference between the actual and ideal weights [106]. If gentamicin will be used in combination with a parenteral antimicrobial with activity against anaerobic agents for prophylaxis, it is probably advisable to use $4.5-5 \mathrm{mg} / \mathrm{kg}$ as a single dose [16]. This dose of gentamicin has been found safe and effective in a large body of literature examining the use of single daily doses of gentamicin for therapeutic indications [106-113]. When used as a single dose for prophylaxis, the risk of toxicity from gentamicin is very low.
Obese patients are often underrepresented in clinical trials and are not currently considered a special population for whom FDA requires separate pharmacokinetic studies during antimicrobial research and development by the drug manufacturer. Obesity has been recognized as a risk factor for SSI; therefore, optimal dosing of antimicrobial prophylaxis is needed in these patients [114]. While a BMI of $>30 \mathrm{~kg} / \mathrm{m}^{2}$ is commonly used to define obesity, the body fat percentage ( $>25 \%$ in men and $>31 \%$ in women) may better predict SSI risk, because the BMI may not reflect body composition. In a recent prospective cohort study of 590 patients undergoing elective surgery, there was no significant difference in SSI rates in nonobese and obese patients when the BMI was used to define obesity $(12.3 \%$ versus $11.6 \%$, respectively) [115]. However, when the body fat percentage (determined by bioelectrical impedance analysis) was used as the basis for identifying obesity ( $>25 \%$ in men and $>31 \%$ in women), obese patients had a five-fold-higher risk of SSI than did nonobese patients (OR, 5.3; 95\% CI, 1.2-23.1; $p=0.03)$. These findings suggest that body fat percentage is a more sensitive and precise measurement of SSI risk than is the BMI.

Redosing. Intraoperative redosing is needed to ensure adequate serum and tissue concentrations of the antimicrobial if the duration of the procedure exceeds two halflives of the antimicrobial or there is excessive blood loss (i.e., $>1,500 \mathrm{~mL}$ ) $[17,41,94,116-121]$. The redosing interval should be measured from the time of administration of the preoperative dose, not from the beginning of the procedure. Redosing may also be warranted if there are factors that shorten the half-life of the antimicrobial agent (e.g., extensive burns). Redosing may not be warranted in patients in whom the half-life of the antimicrobial agent is prolonged (e.g., patients with renal insufficiency or renal failure). See Table 1 for antimicrobial-specific redosing recommendations.

\section{Duration}

The shortest effective duration of antimicrobial administration for preventing SSI is not known; however, evidence is mounting that postoperative antimicrobial administration is not necessary for most procedures $[6,7,41,122-124]$. The duration of antimicrobial prophylaxis should be less than 24 hours for most procedures. Cardiothoracic procedures for which a prophylaxis duration of up to 48 hours has been accepted without evidence to support the practice is an area that remains controversial. The duration of cardiothoracic prophylaxis in these guidelines is based on expert panel consensus because the available data do not delineate the optimal duration of prophylaxis. In these procedures, prophylaxis for the duration of the procedure and certainly for less than 24 hours is appropriate.

A 1992 meta-analysis of studies comparing first-generation cephalosporins and antistaphylococcal antimicrobials (e.g., penicillins) with second-generation cephalosporins in patients undergoing cardiothoracic surgery found a reduction in the rate of SSI with second-generation cephalosporins but no benefit from continuing surgical prophylaxis beyond $48 \mathrm{~h}$ [125]. Reports published in 1980 [126], 1993 [127], 1997 [128], and 2000 [129] involving seven studies that compared singledose prophylaxis or prophylaxis only during the operation with durations of one to four days failed to show any reduction 
in SSIs with the longer durations of prophylaxis. In a morerecent observational four-year cohort study of 2,641 patients undergoing coronary artery bypass graft (CABG) surgery, the extended use of antimicrobial prophylaxis ( $>48 \mathrm{~h}$ ) instead of a shorter duration of prophylaxis $(<48 \mathrm{~h})$ failed to reduce the risk of SSI (OR, 1.2; 95\% CI, 0.8-1.6) [130]. Moreover, prolonged prophylaxis was associated with an increased risk of acquired antimicrobial resistance (cephalosporin-resistant Enterobacteriaceae and VRE) compared with short-term prophylaxis (OR, 1.6; 95\% CI, 1.1-2.6). There are no data to support the continuation of antimicrobial prophylaxis until all indwelling drains and intravascular catheters are removed [19,31,32,41,131-134].

\section{Topical administration of irrigations, pastes, and washes}

Intravenous and oral antimicrobial administration are the main focus of these guidelines, and these routes of administration are used for most surgical procedures addressed by these guidelines, with the exception of ophthalmic procedures, for which topical administration is the primary route of administration. Limited high-quality data are available regarding the use of antimicrobial irrigations, pastes, and washes that are administered topically. Studies published in the early 1980s demonstrated that prophylactic topical administration of antimicrobials in the surgical incision during various nonophthalmic procedures is superior to placebo but not superior to parenteral administration, and topical administration does not increase the efficacy of parenteral antimicrobials when used in combination for prophylaxis [135-138]. Additional highquality data on the safety and efficacy of topical antimicrobial administration as an adjunct to i.v. administration are needed to determine the role of topical antimicrobial prophylaxis.

One area of interest for topical administration of antimicrobials, mainly gentamicin and vancomycin, is application to the sternum during cardiac procedures in combination with i.v. agents to prevent mediastinitis. This strategy has been evaluated in cohort and randomized controlled studies [139-142]. While the studies found a significantly lower rate of SSI with topical antimicrobials compared with standard prophylaxis [140], placebo [142], and a historical control [139], a smaller, randomized, placebo-controlled study found no difference between groups [141].

More recently, implantable gentamicin collagen sponges failed to show any efficacy in reducing SSIs in a large prospective study of patients undergoing cardiac surgery and resulted in an increased infection rate in patients undergoing colectomy $[143,144]$. The safety and efficacy of topical antimicrobials have not been clearly established; therefore, routine use of this route cannot be recommended in cardiac or other procedures [145]

\section{Preoperative screening and decolonization}

Staphylococcus aureus is the most common pathogen causing SSIs, accounting for $30 \%$ of SSIs in the United States. Colonization with $S$. aureus, primarily in the nares, occurs in roughly one in four persons and increases the risk of SSI by 2- to 14-fold [146-152]. A national survey assessing nasal colonization with $S$. aureus in the general population conducted from 2001 through 2004 found that while the rate of colonization with $S$. aureus decreased from $32.4 \%$ in $2001-02$ to $28.6 \%$ in $2003-04$ $(p<0.01)$, the rate of colonization with MRSA increased from $0.8 \%$ to $1.5 \%(p<0.05)[75]$.
Preoperative screening for S. aureus carriage and decolonization strategies have been explored as means to reduce the rate of SSIs. Anterior nasal swab cultures are most commonly used for preoperative surveillance, but screening additional sites (pharynx, groin, wounds, rectum) can increase detection rates [153]. Such preoperative surveillance swabs that can be cultured on selective or nonselective media or sent for rapid polymerase chain reaction (PCR)-based screening can be used to identify colonized patients in the preoperative period. When properly used, all of these techniques can identify MSSA and MRSA. However, not all PCR-based systems will identify both MRSA and MSSA so verification with the laboratory is needed. While many studies have focused specifically on MRSA screening in high-risk hospitalized patients in an effort to prevent MRSA SSI and hospital-acquired infections, the risk of developing an SSI remains elevated for any $S$. aureus carrier. While some authors advocate screening for MRSA carriage in the general population, the data supporting universal screening in the surgical population are more controversial [154,155]. Screening has been advocated to both identify candidates for $S$. aureus decolonization and inform the selection of optimal prophylactic antimicrobials, such as the addition of vancomycin for those colonized with MRSA.

FDA has approved intranasal mupirocin to eradicate MRSA nasal colonization in adult patients and health care workers [156]. It is noted in the prescribing information that there are insufficient data to support use in prevention of autoinfection of high-risk patients from their own nasal colonization with $S$. aureus. However, additional data have demonstrated that the use of intranasal mupirocin in nasal carriers of $S$. aureus decreases the rate of $S$. aureus infections $[157,158]$. One metaanalysis of seven studies focused on surgical patients only [157]; the other meta-analysis of nine studies included highquality studies in dialysis patients [158].

Recent studies have confirmed that $S$. aureus decolonization of the anterior nares decreases SSI rates in many surgical patients [159]. The data are most compelling in cardiac and orthopedic surgery patients. There are fewer data in general surgery patients. A large, randomized controlled trial of general, cardiac, and neurosurgical patients $(n=3,864)$ revealed that prophylactic intranasal application of mupirocin did not significantly reduce the overall rate of S. aureus SSIs $(2.3 \%$ in the mupirocin group versus $2.4 \%$ in the control group) but did decrease the rate of $S$. aureus SSI among S. aureus carriers $(3.7 \%$ in the mupirocin group versus 5.9\% in the control group) [160].

Another randomized controlled trial found no significant difference in the rate of postoperative S. aureus SSIs among cardiac surgery patients receiving intranasal mupirocin and those receiving placebo, but the study was limited by the small numbers of patients $(n=257)$ and reported SSIs $(n=5)$ [161]. Among elective orthopedic patients undergoing implantation and other procedures, a randomized clinical trial demonstrated a nonsignificant reduction in the rate of postoperative S. aureus SSIs in patients receiving mupirocin $(n=315,3.8 \%)$ compared with those receiving placebo $(n=299,4.7 \%)$ [150].

A recent randomized, double-blind, placebo-controlled, multicenter study conducted in The Netherlands found that the use of mupirocin nasal ointment and chlorhexidine baths in identified $S$. aureus carriers reduced the risk of hospitalassociated $S$. aureus infections [162]. In the study, a real-time PCR assay was used to rapidly identify $S$. aureus nasal carriers; all of the $S$. aureus isolates were susceptible to methicillin. Deep 
SSIs occurred in $0.9 \%$ of the mupirocin-chlorhexidine-treated group (4 of 441 patients) versus $4.4 \%$ of the placebo group (16 of 367 patients) (relative risk, $0.21 ; 95 \%$ CI, 0.07-0.62). The reduction in superficial SSIs was less marked $(1.6 \%$ versus $3.5 \%$; relative risk, $0.45 ; 95 \% \mathrm{CI}, 0.18-1.11)$. It is plausible that this approach would be beneficial in a setting of MRSA, but it has not been proven.

Most studies conclude that the use of preoperative intranasal mupirocin in colonized patients is safe and potentially beneficial as an adjuvant to i.v. antimicrobial prophylaxis to decrease the occurrence of SSIs. However, the optimal timing and duration of administration are not standardized. In most studies, mupirocin was used for five days before the operation. While $S$. aureus resistance to mupirocin has been detected [148,162], raising concerns about the potential for widespread problems with resistance from routine use of this agent, resistance has only rarely been seen in the preoperative setting. Low-level resistance is associated with an increased rate of failure of decolonization and has been seen in institutions that use standardized mupirocin decolonization protocols [163]. Therefore, when decolonization therapy (e.g., mupirocin) is used as an adjunctive measure to prevent S. aureus SSI, surveillance of susceptibility of S. aureus isolated from SSIs to mupirocin is recommended [164]. While universal use of mupirocin is discouraged, specific recommendations for the drug's use can be found in the cardiac and orthopedic sections of these guidelines.

\section{Future research}

Additional research is needed in several areas related to surgical antimicrobial prophylaxis. The risks and benefits of continuing antimicrobial prophylaxis after the conclusion of the operative procedure, including dosing and duration, need to be further evaluated. Insight is needed to make specific recommendations for intraoperative repeat dosing, weightbased dosing in obese patients, and timing of presurgical antimicrobials that must be administered over a prolonged period (e.g., vancomycin, fluoroquinolones). Additional clarification is needed regarding targeted antimicrobial concentrations and intraoperative monitoring of antimicrobial serum and tissue concentrations to optimize efficacy. The role of topical administration of antimicrobial agents as a substitute for or an adjunct to i.v. antimicrobial prophylaxis needs to be further evaluated. Additional data are needed to guide the selection of antimicrobial agents for prophylaxis, particularly combination regimens, for patients with allergies to $\beta$-lactam antimicrobials. Data are also needed to devise strategies to optimize antimicrobial prophylaxis in patients and facilities with a high risk or high prevalence of resistant organisms implicated in SSIs (e.g., MRSA). Optimal strategies for screening for S. aureus and decolonization for certain procedures need to be identified. Finally, outcomes studies are needed to assess the impact of using quality measures and pay-for-performance incentives designed to reduce surgical morbidity and mortality.

\section{Cardiac procedures}

\section{Background}

Cardiac procedures include CABG procedures, valve repairs, and placement of temporary or permanent implantable cardiac devices, including ventricular assist devices (VADs).
SSIs, including mediastinitis and sternal SSI, are rare but serious complications after cardiac procedures. In patients undergoing $\mathrm{CABG}$, the mean frequency of SSIs depending on NHSN SSI risk index category ranges from 0.35 to 8.49 per 100 operations when donor sites are included [165]. The mean frequency of SSIs depending on NHSN SSI risk index category for patients undergoing CABG with only chest incisions ranges from 0.23 to 5.67 per 100 operations [165]. Most of these infections are superficial in depth. Patientrelated and procedure-related risk factors for SSIs after cardiac procedures have been identified from several singlecenter cohort and case-control studies [117,128,166-176]. These include diabetes [166,169,171-175], hyperglycemia [177-182], peripheral vascular disease [171,172,174], chronic obstructive pulmonary disease [166,174,175], obesity (BMI of $>30 \mathrm{~kg} / \mathrm{m}^{2}$ ) [166-168,171,173-176], heart failure [171,172], advanced age $[117,128,166,172]$, involvement of internal mammary artery [168-172], reoperation [169-171], increased number of grafts [171], long duration of surgery [117,166, $167,176]$, and S. aureus nasal colonization [146,160].

Patients requiring extracorporeal membrane oxygenation (ECMO) as a bridge to cardiac or lung transplantation should be treated with a similar approach. If there is no history of colonization or previous infection, the general recommendations for SSI antimicrobial prophylaxis for the specific procedure should be followed. For ECMO patients with a history of colonization or previous infection, changing the preoperative antimicrobial prophylaxis to cover these pathogens must be considered, weighing whether the pathogen is relevant to SSIs in the planned procedure.

\section{Organisms}

Almost two thirds of organisms isolated in both adult and pediatric patients undergoing cardiac procedures are grampositive, including $S$. aureus, coagulase-negative staphylococcus, and, rarely, Propionibacterium acnes. Gram-negative organisms are less commonly isolated in these patients and include Enterobacter species, Pseudomonas aeruginosa, Escherichia coli, Klebsiella pneumoniae, Proteus mirabilis, and Acinetobacter species [93,139,146,183-192].

\section{Efficacy}

The SSI rate in cardiac procedures is low, but there are potential consequences if infection occurs. Multiple studies have found that antimicrobial prophylaxis in cardiac procedures lowers the occurrence of postoperative SSI up to five-fold [125].

Choice of agent. Cephalosporins have been the most studied antimicrobials for the prevention of SSIs in cardiac procedures. Both first-generation (cefazolin) and secondgeneration (cefamandole and cefuroxime) cephalosporins have been shown to be effective in reducing SSI in cardiac surgery; however, the superiority of one class over another has not been proven [125,127,193-199].

A meta-analysis comparing cephalosporins with glycopeptides (e.g., vancomycin) as antimicrobial prophylaxis regimens for cardiac procedures found a higher frequency of postoperative chest and deep-chest SSIs and a trend toward an increased risk of gram-positive SSI in the glycopeptide group but a lower frequency of SSIs caused by resistant grampositive pathogens [72]. The routine use of vancomycin for the 
prevention of SSIs is not recommended, based on limited evidence of efficacy and concerns of increased glycopeptide resistance of microorganisms $[8,116]$. There is no clear evidence to support the use of vancomycin, alone or in combination with other antimicrobials, for routine antimicrobial prophylaxis in institutions that have a high prevalence of MRSA $[8,11,41,72,73,116,200]$. Vancomycin should be considered in patients who are colonized with MRSA [41,116,201]. The accepted alternative antimicrobial for $\beta$-lactam-allergic patients undergoing cardiac procedures is vancomycin or clindamycin for gram-positive coverage $[41,116,201,202]$. The addition of an aminoglycoside, aztreonam, or a fluoroquinolone may be prudent when gram-negative pathogens are a concern $[8,116]$.

Mupirocin. The proportion of infections related to S. aureus among patients undergoing cardiac surgery and the increase in MRSA as a cause of SSIs at some institutions have led to investigations of methods for preoperative eradication, particularly with intranasal mupirocin [203]. Readers are referred to the Common Principles section of these guidelines for discussion of the use of intranasal mupirocin. Of note, the data demonstrated a $45 \%$ reduction in S. aureus SSIs with the use of preoperative mupirocin among patients known to be colonized with S. aureus who undergo cardiac procedures [157,193]. Institutions should monitor for mupirocin resistance periodically.

Topical administration. Additional information on topical administration of antimicrobials can be found in the Common Principles section of these guidelines. Use of topical antimicrobials, mainly gentamicin or vancomycin, applied to the sternum during cardiac procedures in combination with i.v. agents to prevent mediastinitis has been evaluated in both cohort [139] and randomized controlled studies [140-142]. While the studies found a significantly lower rate of SSIs with topical antimicrobials compared with standard prophylaxis [140], placebo [142], and a historical control [139], a smaller randomized, placebocontrolled study found no difference between groups [141]. More recent studies of gentamicin collagen sponges failed to show any efficacy in a large prospective study of cardiac surgery [143]. The safety and efficacy of topical antimicrobials have not been clearly established and therefore cannot be recommended for routine use in cardiac procedures [139-142].

Cardiopulmonary bypass. Cardiopulmonary bypass $(\mathrm{CPB})$ is a common surgical technique in cardiac procedures that alters the volume of distribution and bioavailability of medications administered during the procedure $[116,204,205]$. Several small cohort or comparative studies [128,204-213] have evaluated the serum and tissue concentrations of several routinely used antimicrobial prophylactic agents (i.e., cefazolin, cefuroxime, gentamicin, and vancomycin) in patients undergoing CPB during cardiac procedures. Until further clinical outcomes data and well-designed studies become available to inform alternative dosing strategies, routinely used doses of common antimicrobial agents should be used in patients undergoing $\mathrm{CPB}$ during cardiac procedures.

Duration. The optimal duration of antimicrobial prophylaxis for cardiac procedures continues to be evaluated. Data support a duration ranging from a single dose up to $24 \mathrm{~h}$ postoperatively $[41,99,131,191,214-217]$. No significant differences were found in several small studies in patients un- dergoing cardiac procedures between these dosing strategies in patients primarily receiving first- or second-generation cephalosporins. Although a recent meta-analysis suggested the possibility of increased efficacy with cardiac surgical prophylaxis extending beyond $24 \mathrm{~h}$, the authors noted that the findings were limited by the heterogeneity of antimicrobial regimens used and the risk of bias in the published studies [218]. The comparisons of varying durations were performed with different antimicrobials with differing efficacy and do not support longer durations. Consequently, this meta-analysis does not provide evidence to support changing the currently accepted prophylaxis duration of less than 24 hours, particularly given the evidence from studies involving noncardiac operations. The currently accepted duration of prophylaxis for cardiac procedures is less than $24 \mathrm{~h}$, but prophylaxis should be continued for the duration of the procedure [41,59,126-129,131,201].

Two small studies did not support the continuation of antimicrobial prophylaxis until intravascular catheters or intraaortic balloon pumps were removed, due to a lack of influence on infections or catheter colonization compared with short-course (24 hours) cefazolin or cefuroxime $[219,220]$. The practice of continuing antimicrobial prophylaxis until all invasive lines, drains, and indwelling catheters are removed cannot be supported due to concerns regarding the development of drug-resistant organisms, superinfections, and drug toxicity $[41,131]$.

\section{Pediatric efficacy}

The rate of SSI in pediatric cardiac procedures is sometimes higher than in adult patients $[20,31,221]$. Significant risk factors in pediatric patients with a mediastinal SSI included the presence of other infections at the time of the procedure, young age (newborns and infants), small body size, the duration of the procedure (including CPB time), the need for an intraoperative blood transfusion, an open sternum postoperatively, the need for a reexploration procedure, the length of stay in the intensive care unit, an NNIS/NHSN risk score of 2, and the performance of emergency procedures [20,31,221].

The organisms of concern in pediatric patients are the same as those in adult patients $[20,21,31,221]$. However, MRSA is rarely a concern in this population as a risk factor for SSI [221]. Pediatric patients considered at high risk for MRSA infection are those with preoperative MRSA colonization or a history of MRSA infection, neonates younger than one month of age, and neonates under three months of age who have been in the hospital since birth or have a complex cardiac disorder [21]. Strategies such as intranasal mupirocin and changes in antimicrobial prophylactic agent to vancomycin led to decreased rates of MRSA carriage and the absence of MRSA infections in one time-series evaluation; however, the overall clinical impact of these efforts is still unclear [21,221].

No well-controlled studies have evaluated the efficacy of antimicrobial prophylaxis in pediatric patients undergoing cardiac procedures. Therefore, the efficacy of antimicrobial prophylaxis is extrapolated from adult studies and should be considered the standard of care for pediatric cardiac surgery patients [19].

No well-designed studies or consensus has established the appropriate doses for common antimicrobial prophylactic agents for use in pediatric cardiac patients. Antibiotic doses 
have been extrapolated from guidelines for the prevention of bacterial endocarditis [11]. In recent evaluations, doses of cefazolin have ranged from 25 to $50 \mathrm{mg} / \mathrm{kg}$ [19-21,31], and vancomycin doses have ranged from 10 to $20 \mathrm{mg} / \mathrm{kg}$ [1921,31,222-226]. Gentamicin doses used in studies have included 2.5 [20] and $5 \mathrm{mg} / \mathrm{kg}$ [22]; however, the study authors [22] felt that the higher dose was excessive. The expert panel recognizes that the usual total daily dose for pediatric patients older than six months can be $6.5-7.5 \mathrm{mg} / \mathrm{kg}$ and that dosing schedules for younger patients may be complicated.

\section{Recommendations}

For patients undergoing cardiac procedures, the recommended regimen is a single preincision dose of cefazolin or cefuroxime with appropriate intraoperative redosing (Table 2). Currently, there is no evidence to support continuing prophylaxis until all drains and indwelling catheters are removed. Clindamycin or vancomycin is an acceptable alternative in patients with a documented $\beta$-lactam allergy. Vancomycin should be used for prophylaxis in patients known to be colonized with MRSA. If organizational SSI surveillance shows that gramnegative organisms cause infections for patients undergoing these operations, practitioners should combine clindamycin or vancomycin with another agent (cefazolin if the patient is not $\beta$-lactam-allergic; aztreonam, aminoglycoside, or single-dose fluoroquinolone if the patient is $\beta$-lactam-allergic). Mupirocin should be given intranasally to all patients with documented $S$. aureus colonization. (Strength of evidence for prophylaxis =A.)

\section{Cardiac device insertion procedures}

\section{Background}

Antimicrobial prophylaxis is the standard of care for patients undergoing cardiac implantable device insertion (e.g., pacemaker implantation) [227]. Based on available data and perceived infection risk, antimicrobial prophylaxis is not routinely recommended for cardiac catheterization or transesophageal echocardiogram [228].

The NHSN has reported a mean SSI rate after pacemaker placement of 0.44 per 100 procedures [165]. This rate may underestimate the risk of late SSI and complications [229]. Risk factors for device-related infection after implantation of cardioverter-defibrillator systems or pacemakers identified in two large, prospective, multicenter cohort studies [230,231] and a large case-control study [232] included fever within $24 \mathrm{~h}$ before implantation, temporary pacing before implantation, and early reintervention for hematoma or lead replacement [230]; corticosteroid use for more than one month during the preceding year and more than two leads in place compared with two leads [232]; and development of pocket hematoma [231]. In all of the evaluations, antimicrobial prophylaxis was found to be protective against device-related infection [230232]. Limited data are available on the efficacy and optimal dose and duration of antimicrobial prophylaxis in patients undergoing implantation of a new pacemaker, pacing system, or other cardiac device.

A meta-analysis of 15 prospective, randomized, controlled, mainly open-label studies evaluated the effectiveness of systemic antimicrobial prophylaxis compared with controls (no antimicrobials) on infection rates after pacemaker implantation [227]. Antibiotics included penicillins or cepha- losporins with a duration ranging from a single preoperative dose to four days postoperatively. A consistent and significant protective effect of antimicrobial prophylaxis was found and encouraged the routine use of antimicrobial prophylaxis in patients undergoing permanent pacemaker implantation. A prospective, single-center cohort study found a low rate $(1.7 \%)$ of SSI complications with a single $2 \mathrm{~g}$ dose of cefazolin in patients undergoing implantation of a new pacemaker, pulse-generator replacement, or upgrading of a preexisting pacing system [233]. A notable limitation of the study was the exclusion of patients with temporary percutanous cardiac stimulators who are at high risk of infection.

A large, randomized, double-blind, placebo-controlled study found a significantly lower rate of SSI with a single $1 \mathrm{~g}$ dose of cefazolin $(0.64 \%)$ compared with placebo $(3.28 \%)$ $(\mathrm{p}=0.016)$ given immediately before device implantation or generator replacement in a permanent pacemaker, implantable cardioverter defibrillator, or cardiac resynchronization device in a surgical operating room [231]. The expert panel noted that the cefazolin dose was not adjusted for patient weight. Recently, AHA produced evidence-based guidelines that recommend the use of a single dose of a preoperative antimicrobial [229].

Ventricular assist devices are increasingly used to bridge patients to transplantation or to support individuals who do not respond to medical therapy for congestive heart failure. Very limited data exist on infection rates, and there are no published studies that demonstrate the effectiveness of preoperative antimicrobial therapy. Using 2006-08 data from the Interagency Registry for Mechanically Assisted Circulatory Support, Holman and colleagues [234] reported that most infections related to mechanical cardiac support devices were bacterial $(87 \%)$, with the remainder associated with fungal $(9 \%)$, viral $(1 \%)$, protozoal $(0.3 \%)$, or unknown $(2 \%)$ causes. Driveline infections are primarily caused by staphylococcal species from the skin. Fungal organisms also play an important role in VAD infections, most notably Candida species, and carry a high risk of mortality. A recent survey of antimicrobial surgical prophylaxis with VADs illustrates the variability and lack of consensus with regimens, using anywhere from one to four drugs for a duration of $24-72 \mathrm{~h}$ [235]. Immediate postoperative infections are caused by gram-positive organisms. Complications from long-term infections should not be confused with immediate postprocedure SSIs [236]. Based on the consensus of the expert panel, antimicrobial prophylaxis for replacement of a VAD due to ongoing or recent infection should incorporate coverage directed at the offending organism or organisms. While many centers use vancomycin plus ciprofloxacin plus fluconazole, this practice is not based on the published evidence.

\section{Recommendation}

A single dose of cefazolin or cefuroxime is recommended for device implantation or generator replacement in a permanent pacemaker, implantable cardioverter defibrillator, or cardiac resynchronization device. (Strength of evidence for prophylaxis $=$ A.) There is limited evidence to make specific recommendations for VADs, and each practice should tailor protocols based on pathogen prevalence and local susceptibility profiles. Clindamycin or vancomycin is an acceptable alternative in patients with a documented $\beta$-lactam allergy. 
Vancomycin should be considered for prophylaxis in patients known to be colonized with MRSA.

\section{Thoracic procedures}

\section{Background}

Noncardiac thoracic procedures include lobectomy, pneumonectomy, thoracoscopy, lung resection, and thoracotomy. In addition to SSIs, postoperative nosocomial pneumonia and empyema are of concern after thoracic procedures [237].

NHSN has reported that the rate of infection associated with thoracic surgery ranges from $0.76 \%$ to $2.04 \%$ [165]. Studies have found that the reported rate of SSIs after thoracic procedures in patients receiving antimicrobial prophylaxis ranged from $0.42 \%$ to $4 \%$ [238-241]. One study found an SSI rate of $14 \%$ when prophylaxis was not used [239]. The reported rates of pneumonia and empyema with antimicrobial prophylaxis are $3-24 \%$ and $0-7 \%$, respectively $[237,239-244]$.

Video-assisted thoracoscopic surgery (VATS) is commonly used for thoracic procedures. In some settings, VATS constitutes one third or more of all thoracic surgical procedures [245]. Since VATS uses small incisions, the rate of SSIs is lower compared with the rate associated with open thoracic surgical procedures [246]. A prospective cohort study $(n=346)$ confirmed a low rate of SSIs (1.7\%) after minimally invasive VATS procedures [240]. An additional prospective study of 988 lung resection patients confirmed that the SSI rate was significantly lower $(5.5 \%)$ in VATS patients than in open thoracotomy patients $(14.3 \%)$ [247]. Furthermore, SSI correlated with the duration of surgery, serum albumin, concurrent comorbidity, age, and forced expiratory volume in one second. Antimicrobial prophylaxis recommendations in this section refer to both open thoracotomy and VATS procedures. Based on available data and perceived infection risk, antimicrobial prophylaxis is not routinely recommended for chest tube insertion.

Results of a prospective cohort and case-control study revealed the following independent risk factors for pneumonia after thoracic procedures: extent of lung resection, intraoperative bronchial colonization, chronic obstructive pulmonary disease, BMI of $>25 \mathrm{~kg} / \mathrm{m}^{2}$, induction therapy (chemotherapy, radiotherapy, or chemoradiotherapy), advanced age ( $\geq 75$ years old), and stage III or IV cancer [243,244].

\section{Organisms}

The organisms reported from SSIs in patients undergoing thoracic procedures were S. aureus and S. epidermidis [237]. Organisms isolated in patients with postoperative pneumonia included gram-positive (Streptococcus and Staphylococcus species), gram-negative (Haemophilus influenzae, Enterobacter cloacae, K. pneumoniae, Acinetobacter species, P. aeruginosa, and Moraxella catarrhalis), and fungal (Candida species) pathogens [237,239-243].

\section{Efficacy}

Antimicrobial prophylaxis is the standard of care for patients undergoing noncardiac thoracic surgery, including pulmonary resection $[11,201,237]$. One randomized, doubleblind, placebo-controlled, single-center study of patients in Spain undergoing pulmonary resection, persistent pneumothorax without thoracotomy tube before surgery, and nonpulmonary thoracic surgical procedures, excluding those involving the esophagus and exploratory thoracotomies, compared a single dose of cefazolin $1 \mathrm{~g}$ i.v. and placebo given 30 minutes before the procedure [239]. The study was stopped early due to the significant difference in SSI rates between groups $(1.5 \%$ with cefazolin versus $14 \%$ with placebo, $\mathrm{p}<0.01)$. No differences in the rates of pneumonia and empyema were seen between groups, but these were not endpoints of the study.

Choice of agent. There is no clear optimal choice for antimicrobial prophylaxis in thoracic procedures. The need to consider pneumonia and empyema as well as SSIs after thoracic procedures has been raised in the literature $[237,241-$ 244]. There are a limited number of small, single-center, randomized controlled or cohort studies that evaluated several antimicrobial agents. One small, randomized controlled study and one cohort study found that ampicillin-sulbactam was significantly better than cephalosporins (cefazolin and cefamandole) for preventing pneumonia [242,243]. No statistically significant difference was found between cefuroxime and cefepime in the rate of postoperative SSI, pneumonia, or empyema in a small, randomized controlled study in patients undergoing elective thoracotomy [241]. Lower rates of infections and susceptibility of all organisms were noted with cefuroxime compared with cefepime. Therefore, the study authors concluded that cefuroxime was marginally more effective and was more cost-effective than cefepime.

Duration. No clear consensus on the duration of antimicrobial prophylaxis has been established. Studies have evaluated different dosing strategies for cephalosporins or penicillins, with most studies using single doses given preoperatively within 60 min before surgical incision [237,239,240,242,244]. Studies found differing results when comparing agents given for $24 \mathrm{~h}$ (cefepime, ampicillin-sulbactam) and $48 \mathrm{~h}$ (cefuroxime, cefamandole); however, these findings may be attributable to the different antimicrobials tested $[241,243]$. Additional discussion on dosing is provided in the Common Principles section of these guidelines.

\section{Recommendations}

In patients undergoing thoracic procedures, a single dose of cefazolin or ampicillin- sulbactam is recommended (Appendix B). Clindamycin or vancomycin is an acceptable alternative in patients with a documented $\beta$-lactam allergy. Vancomycin should be used for prophylaxis in patients known to be colonized with MRSA. If organizational SSI surveillance shows that gram-negative organisms are associated with infections during these operations or if there is risk of gram-negative contamination of the surgical site, practitioners should combine clindamycin or vancomycin with another agent (cefazolin if the patient is not $\beta$-lactam-allergic; aztreonam, aminoglycoside, or single-dose fluoroquinolone if the patient is $\beta$-lactam-allergic). (Strength of evidence for prophylaxis for VATS $=\mathrm{C}$; strength of evidence for prophylaxis for other thoracic procedures $=\mathrm{A}$.)

\section{Gastroduodenal procedures}

\section{Background}

The gastroduodenal procedures considered in these guidelines include resection with or without vagotomy for 
gastric or duodenal ulcers, resection for gastric carcinoma, revision required to repair strictures of the gastric outlet, percutaneous endoscopic gastrostomy (PEG) insertion, perforated ulcer procedures (i.e., Graham patch repair), pancreaticoduodenectomy (Whipple procedure), and bariatric surgical procedures (gastric bypass, gastric banding, gastroplasty, other restrictive procedures, biliopancreatic diversion). Studies specifically addressing antimicrobial prophylaxis for gastroesophageal reflux disease procedures (Nissen fundoplication) or highly selective vagotomy for ulcers (usually done laparoscopically) could not be identified. Antireflux procedures and highly selective vagotomy are clean procedures in contrast to essentially all other gastroduodenal procedures that are clean-contaminated. Other procedures that are generally performed using laparoscopic or endoscopic techniques (e.g., endoscopic retrograde cholangiopancreatography) are not specifically discussed in this document. Natural orifice transluminal endoscopic surgery (NOTES) is a developing operative technique using natural orifices (e.g., vagina, anus, mouth, stomach) for entry into the abdomen that leaves no visible scar [248]. No studies on antimicrobial prophylaxis using NOTES have been published. SSI rates reported in patients not receiving antimicrobial prophylaxis were $6 \%$ after vagotomy and drainage, $13 \%$ after gastric ulcer procedures, $6.8-17 \%$ after procedures for gastric cancer [249-253], 8\% for pancreaticoduodenectomy [254], and $23.9-26 \%$ after PEG insertion [255,256].

The stomach is an effective barrier to bacterial colonization; this is at least partially related to its acidity. The stomach and the duodenum typically contain small numbers of organisms $\left(<10^{4}\right]$ colony-forming units $\left.[\mathrm{CFU}] / \mathrm{mL}\right)$, the most common of which are streptococci, lactobacilli, diphtheroids, and fungi $[257,258]$. Treatment with agents that increase gastric $\mathrm{pH}$ increases the concentration of gastric organisms [259-261]. Alterations in gastric and duodenal bacterial flora as a result of increases in gastric $\mathrm{pH}$ have the potential to increase the postoperative infection rate [262,263].

The risk of postoperative infection in gastroduodenal procedures depends on a number of factors, including the gastroduodenal procedure performed. Patients who are at highest risk include those with achlorhydria, including those receiving pharmacotherapy with histamine $\mathrm{H}_{2}$-receptor antagonists or proton-pump inhibitors [264], gastroduodenal perforation, decreased gastric motility, gastric outlet obstruction, morbid obesity, gastric bleeding, or cancer [265]. Similar to other types of surgical procedures, risk factors for SSIs related to gastroduodenal procedures include long procedure duration [252,266,267], performance of emergency procedures [250,261], greater than normal blood loss [251,252], American Society of Anesthesiologists (ASA) classification of $\geq 3$, and late administration of antimicrobials [268].

\section{Organisms}

The most common organisms cultured from SSIs after gastroduodenal procedures are coliforms (E. coli, Proteus species, Klebsiella species), staphylococci, streptococci, enterococci, and occasionally Bacteroides species [101,269-276].

\section{Efficacy}

Randomized controlled trials have shown that prophylactic antimicrobials are effective in decreasing postoperative infection rates in high-risk patients after gastroduodenal procedures. The majority of available studies were conducted in single centers outside of the United States. Relative to other types of gastrointestinal tract procedures, the number of clinical trials evaluating antimicrobial prophylaxis for gastroduodenal procedures is limited. In placebo-controlled trials, infection rates ranged from $0 \%$ to $22 \%$ for patients receiving cephalosporins or penicillins and from $1.7 \%$ to $66 \%$ for patients receiving placebo $[270,271,273-275,277-284]$. The difference was significant in most studies.

Data support antimicrobial prophylaxis for patients undergoing PEG insertion [264,285-287]. A Cochrane review of systemic antimicrobial prophylaxis for PEG procedures that included 11 randomized controlled trials and 1,196 patients found a statistically significant reduction in peristomal infections with antimicrobial prophylaxis (OR, 0.35; 95\% CI, 0.23-0.48) [288]. Two meta-analyses found statistically significant decreases in SSIs with antimicrobial prophylaxis compared with placebo or controls, from $23.9-26 \%$ to $6.4-8 \%$, respectively $[255,256]$. Most well-designed, randomized controlled studies found a significant decrease in postoperative SSIs or peristomal infections with single i.v. doses of a cephalosporin or penicillin, ranging from $11 \%$ to $17 \%$, compared with from $18 \%$ to $66 \%$ with placebo or no antimicrobials [ 279 282,288 ]. Conflicting results have been seen in studies evaluating the use of preoperative patient MRSA screening, decontamination washes and shampoos, five-day preoperative treatment with intranasal mupirocin, and single-dose teicoplanin preoperative prophylaxis to decrease postoperative MRSA infections during PEG insertion [289,290].

While there have been no well-designed clinical trials of antimicrobial prophylaxis for patients undergoing bariatric surgical procedures, treatment guidelines support its use based on morbid obesity and additional comorbidities as risk factors for postoperative infections [264,291]. There is no consensus on the appropriate antimicrobial regimen; however, higher doses of antimicrobials may be needed for adequate serum and tissue concentrations in morbidly obese patients $[13,268,291]$.

A notable risk factor for SSIs after esophageal and gastroduodenal procedures is decreased gastric acidity and motility resulting from malignancy or acid-suppression therapy [264,276]. Therefore, antimicrobial prophylaxis is indicated for patients undergoing gastric cancer procedures (including gastrectomy) and gastroduodenal procedures related to gastric and duodenal ulcer disease or bariatric surgery or pancreaticoduodenectomy. Evaluations of practice for pancreaticoduodenectomy show that antimicrobials are typically given due to concerns of bile contamination. Prophylaxis for gastroduodenal procedures that do not enter the gastrointestinal tract, such as antireflux procedures, should be limited to high-risk patients due to lack of data supporting general use in all patients. Furthermore, laparoscopic antireflux procedures are associated with very low SSI rates $(0.3 \%)$ compared with open antireflux procedures $(1.4 \%)$, just as laparoscopic gastric bypass procedures are associated with lower rates than in open procedures (0.4\% versus $1.2 \%$ ) [292].

Choice of agent. The most frequently used agents for gastroduodenal procedures were first-generation [271,273, $277,278,284,293-297]$ and second-generation [269,270,274,275, $280,293,294,298$ ] cephalosporins. No differences in efficacy between first- and second-generation cephalosporins were 
found. Amoxicillin-clavulanate $[279,282,283,299]$ and ciprofloxacin $[269,300]$ were also evaluated with similar results. Relatively few studies have compared the efficacy of different agents in reducing postoperative infection rates.

One meta-analysis recommended using a single dose of an i.v. broad-spectrum antimicrobial for SSI prophylaxis in these patients [256], while another found no differences between penicillin- or cephalosporin-based regimens and three-dose or single-dose regimens [255]. In a comparative study, oral or i.v. ciprofloxacin and i.v. cefuroxime were similarly effective in upper gastrointestinal procedures, including gastrectomy, vagotomy, and fundoplication [300]. No differences in efficacy were seen between ceftriaxone and combination ceftriaxone and metronidazole for PEG insertion in pediatric patients [301]. An open-label study found a significant decrease in local peristomal and systemic infection (i.e., pneumonia) after PEG insertion after a single $1 \mathrm{~g}$ i.v. dose of ceftriaxone was given $30 \mathrm{~min}$ before surgery when compared with placebo $(13.3 \%$ and $36.3 \%$, respectively; $p<0.05)$ [281]. No differences were noted between cefotaxime and piperacillin-tazobactam for PEG SSIs [288]. Ampicillin-sulbactam and cefazolin had equal efficacy in gastrectomy [253]. One study found that piperacillin-tazobactam in combination with ciprofloxacin or gentamicin was the most active regimen against bacteria recovered from bile in pancreatoduodenectomy patients [302].

Duration. The majority of studies evaluated a single dose of cephalosporin or penicillin [256,279-284,288,290,297]. The available data indicate that single-dose and multiple-dose regimens are similarly effective. Three studies compared single- and multiple-dose regimens of cefamandole [294], amoxicillin-cluvulanate [299], and ampicillin-sulbactam and cefazolin [253]. There were no significant differences in SSI rates. Multiple-dose regimens of first-generation (cefazolin) or second-generation (cefotiam) cephalosporins of four days, operative day only, and three days in duration did not differ in overall SSI rates [295].

\section{Recommendations}

Antimicrobial prophylaxis in gastroduodenal procedures should be considered for patients at highest risk for postoperative infections, including risk factors such as increased gastric $\mathrm{pH}$ (e.g., patients receiving acid-suppression therapy), gastroduodenal perforation, decreased gastric motility, gastric outlet obstruction, gastric bleeding, morbid obesity, ASA classification of $\geq 3$, and cancer.

A single dose of cefazolin is recommended in procedures during which the lumen of the intestinal tract is entered (Table 2). (Strength of evidence for prophylaxis = A.) A single dose of cefazolin is recommended in clean procedures, such as highly selective vagotomy, and antireflux procedures only in patients at high risk of postoperative infection due to the presence of the above risk factors. (Strength of evidence for prophylaxis $=\mathrm{C}$.) Alternative regimens for patients with $\beta$-lactam allergy include clindamycin or vancomycin plus gentamicin, aztreonam, or a fluoroquinolone. Higher doses of antimicrobials are uniformly recommended in morbidly obese patients undergoing bariatric procedures. Higher doses of antimicrobials should be considered in significantly overweight patients undergoing gastroduodenal and endoscopic procedures.

\section{Biliary tract procedures}

\section{Background}

Biliary tract procedures include cholecystectomy, exploration of the common bile duct, and choledochoenterostomy. These guidelines pertain only to patients undergoing biliary tract procedures with no evidence of acute biliary tract infection and to patients with community-acquired acute cholecystitis of mild-to-moderate severity. As noted in the Common Principles section, patients receiving therapeutic antimicrobials for an infection before surgery should be given additional antimicrobial prophylaxis before surgery.

These guidelines do not address patients requiring biliary tract procedures for more-severe infections, including community-acquired acute cholecystitis with severe physiological disturbance, advanced age, or immunocompromised state; acute cholangitis; and health-care-associated or nosocomial biliary infections. These biliary tract infections are treated as complicated intraabdominal infections [303]. All patients with a suspected biliary tract infection who undergo biliary tract surgery should receive preoperative i.v. antimicrobials.

The majority of published literature regarding SSIs in biliary tract procedures focuses on cholecystectomy. The overall reported rate of postoperative infection in open biliary tract procedures with antimicrobial prophylaxis is 1-19\% [292,304311]. Infection rates after laparoscopic cholecystectomy range from $0 \%$ to approximately $4 \%$ in patients without antimicrobial prophylaxis $[308,312-320]$ and from $0 \%$ to $7 \%$ with prophylaxis [292,304-323]. Several studies found that laparoscopic cholecystectomy SSI rates were significantly lower than those associated with open cholecystectomy [292,306-311].

Risk factors associated with postoperative SSIs after biliary procedures include performance of emergency procedures [305], diabetes [305,306,311,315,317], longer procedure duration (over 120 minutes) [305,317,324], intraoperative gallbladder rupture [305], age of $>70$ years [6,311,315,317,325], open cholecystectomy $[7,311]$, conversion of laparoscopic to open cholecystectomy [7], higher ASA classification $(\geq 3)$ [306,310,317], episode of biliary colic within 30 days before the procedure $[315,316]$, reintervention in less than a month for noninfectious complications [310], acute cholecystitis $[6,7,306]$, bile spillage [7], jaundice [6,7,306], pregnancy [7], nonfunctioning gallbladder [6], and immunosuppression [7].

The biliary tract is usually sterile. Patients with bacteria in the bile at the time of surgery may be at higher risk of postoperative infection [305,326,327]; however, some studies have found no association between the presence of bacteria in the bile and infection $[305,315,316,319,321]$. Obesity (a BMI of $>30 \mathrm{~kg} / \mathrm{m}^{2}$ ) was found to be a risk factor in some studies [306] but not in others $[315,319]$. Laparoscopic cholecystectomy was associated with a significantly decreased risk for SSI [292,310,324,325].

\section{Organisms}

The organisms most commonly associated with infection after biliary tract procedures include E. coli, Klebsiella species, and enterococci; less frequently, other gram-negative organisms, streptococci, and staphylococci are isolated [305,306,312,315, $316,318,319,321,326,328-338]$. Anaerobes are occasionally reported, most commonly Clostridium species.

Recent studies have documented increasing antimicrobial resistance in the causative pathogens in biliary tract infections 
and other intra-abdominal infections, with up to $40 \%$ of $E$. coli isolates resistant to ampicillin-sulbactam and fluoroquinolones [339-341]. Due to this increasing resistance of E. coli to fluoroquinolones and ampicillin-sulbactam, local population susceptibility profiles should be reviewed to determine the optimal antimicrobials for SSI prevention in biliary tract procedures.

\section{Efficacy}

Numerous studies have evaluated the use of prophylactic antimicrobials during biliary tract procedures, with a focus on laparoscopic cholecystectomy. Laparoscopic cholecystectomy has replaced open cholecystectomy as the standard of practice because of the reduction in recovery time and shorter hospital stay. The majority of studies of antimicrobial prophylaxis for laparoscopic cholecystectomy were underpowered and varied in control groups used (placebo, active, or no treatment), follow-up (from 30 to 60 days, while some studies did not clearly define length of time), and how SSIs were detected and reported [308,312-316,318,319,321,322]. Some studies included patients who were converted from laparoscopic to open cholecystectomy and others did not.

A large, multicenter, quality assurance study in Germany assessed the effectiveness of antimicrobial prophylaxis in laparoscopic and open cholecystectomies [308]. This study included 4,477 patients whose antimicrobial choice and dosage regimens were at the discretion of the medical center and surgeon. Antimicrobials used included first-, second-, and third-generation cephalosporins or penicillins alone or in combination with metronidazole, gentamicin, or both metronidazole and gentamicin. The most common cephalosporin used was ceftriaxone, allowing its data to be separated from data for other antimicrobials. Antimicrobial prophylaxis was administered to 2,217 patients (ceftriaxone $[n=787$ laparoscopic and $n=188$ open] and other antimicrobials [ $n=229$ laparoscopic and $n=229$ open]); none was given to 1,328 laparoscopic and 932 open cholecystectomy patients. Significantly lower overall infectious complications occurred in patients receiving antimicrobial prophylaxis $(0.8 \%$ ceftriaxone and $1.2 \%$ other antimicrobials), compared with $5 \%$ of those who received no prophylaxis $(\mathrm{p}<0.05)$. The overall rates of infectious complications were $0.6 \%, 0.8 \%$, and $3.3 \%$ in patients undergoing laparoscopic cholecystectomy receiving ceftriaxone, other antimicrobials, and no prophylaxis, respectively, and $1.6 \%, 3.9 \%$, and $7.4 \%$, respectively, for patients undergoing open cholecystectomy. Significantly lower rates of SSIs and postoperative pneumonia were noted in patients receiving antimicrobials compared with those who did not receive prophylaxis $(p<0.05)$. Rates of SSI were significantly decreased in laparoscopic cholecystectomy patients who received ceftriaxone $(0.1 \%)$ or other antimicrobials $(0.2 \%)$ compared with those who received no antimicrobial prophylaxis $(1.6 \%)$. SSI rates were significantly decreased in open cholecystectomy patients who received ceftriaxone $(1.0 \%)$ or other antimicrobials (2.6\%) compared with those who received no antimicrobial prophylaxis (4.4\%). The study authors concluded that antimicrobial prophylaxis should be administered to all patients undergoing cholecystectomy, regardless of approach. The study had several limitations, including lack of randomization, lack of adequate controls, and lack of clear definition of patient selection for the antimicrobial regimens. The statistical analysis was not clearly defined.
The study appears to have compared only the use and lack of use of antimicrobials (with ceftriaxone and other antimicrobials combined for analysis) and did not specifically compare the laparoscopic and open approaches.

The findings of this study contrast with those of several other published studies. A meta-analysis of 15 randomized controlled studies evaluated the need for antimicrobial prophylaxis in elective laparoscopic cholecystectomy for patients at low risk of infection [313]. Low risk was defined as not having any of the following: Acute cholecystitis, a history of acute cholecystitis, common bile duct calculi, jaundice, immune suppression, and prosthetic implants. A total of 2,961 patients were enrolled in the studies, including 1,494 who received antimicrobial prophylaxis, primarily with cephalosporins, vancomycin, fluoroquinolones, metronidazole, and amoxicillin-clavulanic acid, and 1,467 controls receiving placebo or no treatment. No significant difference was found in the rates of infectious complications $(2.07 \%$ in patients receiving antimicrobial prophylaxis versus $2.45 \%$ in controls) or SSIs $(1.47 \%$ in patients receiving antimicrobial prophylaxis versus $1.77 \%$ in controls). The authors of the meta-analysis concluded that antimicrobial prophylaxis was not necessary for low-risk patients undergoing elective laparoscopic cholecystectomy. An additional meta-analysis of nine randomized controlled trials $(n=1,437)$ also concluded that prophylactic antimicrobials do not prevent infections in low-risk patients undergoing laparoscopic cholecystectomy [342].

A small, prospective, nonrandomized study compared the use of cefotaxime $1 \mathrm{~g}$ i.v. during surgery with an additional two i.v. doses given eight hours apart after surgery $(n=80)$ with no antimicrobial prophylaxis $(n=86)$ in patients undergoing elective laparoscopic cholecystectomy with accidental or incidental gallbladder rupture and spillage of bile [317]. Patients who had spillage of gallstone calculi or whose operations were converted to open operations were excluded from the study. The rate of SSIs did not significantly differ between treatment groups $(2.5 \%$ with antimicrobials versus $3.4 \%$ without antimicrobial prophylaxis). Based on results of multivariable analysis, routine antimicrobial prophylaxis was not recommended for these patients unless they were diabetic, were older than 60 years, or had an ASA classification of $\geq 3$ or the duration of the procedure exceeded $70 \mathrm{~min}$.

Current data do not support antimicrobial prophylaxis for low-risk patients undergoing elective laparoscopic cholecystectomies or those with incidental or accidental gallbladder rupture. Antimicrobial prophylaxis should be considered for patients at high risk of infection, including those undergoing open cholecystectomy, as described above, or who are considered to be at high risk for conversion to an open procedure.

Choice of agent. The data do not indicate a significant difference among first-, second-, and third-generation cephalosporins. First-generation $[307,308,312,315,319,323,330,336$, $338,343,344]$, second-generation [308,314,315,318,323,327-329, $331,332,335,344-352]$, and third-generation [308,309,315-317, $321,322,332,333,338,349,353,354]$ cephalosporins have been studied more extensively than other antimicrobials. Limited data are available for ampicillin with gentamicin [355], piperacillin [356], amoxicillin-clavulanate [305,338,351,354], ciprofloxacin $[320,333,352,357]$, and cephalosporins or penicillins alone or in combination with metronidazole, gentamicin, or both metronidazole and gentamicin [308]. 
Several studies have compared first-generation cephalosporins with second- or third-generation agents [315,336,338,344$347,353,358]$. With one exception [347], there was no significant difference in efficacy among agents. Other studies found no significant differences in efficacy between ampicillin and cefamandole [335], ciprofloxacin and ceftriaxone [333], amoxicillinclavulanic acid and cefotaxime [354], amoxicillin-clavulanic acid and cefamandole [351], ceftriaxone and ceftazidime [321], and oral and i.v. ciprofloxacin and i.v. cefuroxime [352,357]. One study found that i.v. ampicillin-sulbactam was associated with significantly lower rates of infection compared with cefuroxime [306] and that patients treated with oral ceftibuten had significantly lower infection rates than those who received amoxicillin-clavulanate [338].

Duration. The effect of duration of prophylaxis on outcome has been evaluated. A single dose of a cephalosporin was compared with multiple doses in several studies; no significant differences in efficacy were found [327,329,330, $348,349,353,359]$. The largest study compared one dose of cefuroxime with three doses in 1,004 patients with risk factors for infection who were undergoing biliary tract surgery [327]. There was no significant difference in the rates of minor or major SSIs between the single- and multiple-dose groups. In the majority of studies, one dose of an antimicrobial was administered at induction of anesthesia [306,312,338,352,354], within $30 \mathrm{~min}$ before incision [338], or $1[315,316,320,321]$ or 2 [338] h before incision. Additional doses were given as follows: one dose $12 \mathrm{~h}$ after administration of the initial dose [352], two doses 12 and $24 \mathrm{~h}$ after administration of the initial dose [338], two doses every $6[338]$ or $8[317,319] \mathrm{h}$ after surgery, and one dose $24 \mathrm{~h}$ after surgery [315] and five days after surgery [352]. In one study, a second dose of amoxicillin-clavulanic acid or cefotaxime was administered for procedures lasting longer than $4 \mathrm{~h}$ [354].

\section{Recommendations}

A single dose of cefazolin should be administered in patients undergoing open biliary tract procedures (Table 2). (Strength of evidence for prophylaxis=A.) Alternatives include ampicillin-sulbactam and other cephalosporins (cefotetan, cefoxitin, and ceftriaxone). Alternative regimens for patients with $\beta$-lactam allergy include clindamycin or vancomycin plus gentamicin, aztreonam, or a fluoroquinolone; or metronidazole plus gentamicin or a fluoroquinolone.

Antimicrobial prophylaxis is not necessary in low-risk patients undergoing elective laparoscopic cholecystectomies. (Strength of evidence against prophylaxis for low-risk patients $=$ A.) Antimicrobial prophylaxis is recommended in patients undergoing laparoscopic cholecystectomy who have an increased risk of infectious complications. Risk factors include performance of emergency procedures, diabetes, anticipated procedure duration exceeding $120 \mathrm{~min}$, risk of intraoperative gallbladder rupture, age of $>70$ years, open cholecystectomy, risk of conversion of laparoscopic to open cholecystectomy, ASA classification of $\geq 3$, episode of biliary colic within 30 days before the procedure, reintervention in less than a month for noninfectious complications of prior biliary operation, acute cholecystitis, anticipated bile spillage, jaundice, pregnancy, nonfunctioning gallbladder, and immunosuppression. Because some of these risk factors cannot be determined before the surgical intervention, it may be reasonable to give a single dose of antimicrobial prophylaxis to all patients undergoing laparoscopic cholecystectomy. (Strength of evidence for prophylaxis for high-risk patients =A.)

\section{Appendectomy procedures}

\section{Background}

Cases of appendicitis can be described as complicated or uncomplicated on the basis of the pathology. Patients with uncomplicated appendicitis have an acutely inflamed appendix. Complicated appendicitis includes perforated or gangrenous appendicitis, including peritonitis or abscess formation. Because complicated appendicitis is treated as a complicated intra-abdominal infection [303], it has not been addressed separately in these guidelines. All patients with a suspected clinical diagnosis of appendicitis, even those with an uncomplicated case, should receive appropriate preoperative i.v. antimicrobials for SSI prevention, which, due to the common microbiology encountered, requires similar antimicrobial choices to those used to treat complicated appendicitis.

Approximately $80 \%$ of patients with appendicitis have uncomplicated disease [59]. SSI has been reported in $9-30 \%$ of patients with uncomplicated appendicitis who do not receive prophylactic antimicrobials, though some reports suggest lower complication rates in children with uncomplicated appendicitis [165,360-365]. Mean SSI rates for appendectomy reported in the most recent NHSN report (2006-08) were $1.15 \%$ (60 of 5211) for NHSN risk index categories 0 and 1 versus $3.47 \%$ (23 of 663) for NHSN risk index categories 2 and 3 [165]. Laparoscopic appendectomy has been reported to produce lower rates of incisional (superficial and deep) SSIs than open appendectomy in adults and children in multiple meta-analyses and several randomized clinical trials [292,310,366-371]. However, the rate of organ/space SSIs (i.e., intra-abdominal abscesses) was significantly increased with laparoscopic appendectomy.

\section{Organisms}

The most common microorganisms isolated from SSIs after appendectomy are anaerobic and aerobic gram-negative enteric organisms. Bacteroides fragilis is the most commonly cultured anaerobe, and E. coli is the most frequent aerobe, indicating that the bowel flora constitute a major source for pathogens $[59,372,373]$. Aerobic and anaerobic streptococci, Staphylococcus species, and Enterococcus species also have been reported. Pseudomonas aeruginosa has been reported infrequently.

\section{Efficacy}

Antibiotic prophylaxis is generally recognized as effective in the prevention of postoperative SSIs in patients undergoing appendectomy when compared with placebo [374].

Choice of agent. Randomized controlled trials have failed to identify an agent that is clearly superior to other agents in the prophylaxis of postappendectomy infectious complications. An appropriate choice for SSI prophylaxis in uncomplicated appendicitis would be any single agent or combination of agents that provides adequate gram-negative and anaerobic coverage. The second-generation cephalosporins with anaerobic activity and a first-generation cephalosporin plus metronidazole are the recommended agents on 
the basis of cost and tolerability. Given the relatively equivalent efficacy between agents, a cost-minimization approach is reasonable; the choice of agents should be based on local drug acquisition costs and antimicrobial sensitivity patterns.

A wide range of antimicrobials have been evaluated for prophylaxis in uncomplicated appendicitis. The most commonly used agents were cephalosporins. In general, a secondgeneration cephalosporin with anaerobic activity (cefoxitin or cefotetan) or third-generation cephalosporins with partial anaerobic activity (cefotaxime) were effective, with postoperative SSI rates of $<5 \%$ in most studies [364,375-381].

Piperacillin $2 \mathrm{~g}$ was comparable to cefoxitin $2 \mathrm{~g}$ in a wellcontrolled study [381]. Metronidazole used alone was less effective than cefotaxime, with infection rates above $10 \%$ [376]. However, when metronidazole was combined with cefazolin, ampicillin [382], or gentamicin [378,383], the postoperative SSI rates were $3-6 \%$.

A double-blind, randomized, controlled trial was conducted at two hospitals to evaluate the effect of metronidazole, which is effective against most anaerobes, and cefazolin, which is effective against many aerobic organisms, singly and in combination, on the rate of sepsis after appendectomy [384]. Patients were randomized into one of four groups: metronidazole and placebo, cefazolin and placebo, metronidazole and cefazolin, or double placebo. Patients with generalized peritonitis were excluded for ethical reasons. Treatment was started before the procedure and continued every $8 \mathrm{~h}$ for $24 \mathrm{~h}$. All patients in the trial were followed for about two weeks after discharge from the hospital, and their surgical sites were inspected. A total of 271 patients were assessed. Sepsis rates at the two hospitals were similar. Patients who received both cefazolin and metronidazole had a significantly lower infection rate compared with the other groups [384]. Consistent with the antibacterial spectrum of the agents, a prospective study of antimicrobial prophylaxis for colorectal procedures found that the combination of metronidazole with aztreonam did not show adequate coverage of gram-positive organisms [385]. The Common Principles section of these guidelines provides additional considerations for weight-based dosing.

Duration. In most of the studies of second- or thirdgeneration cephalosporins or metronidazole combinations, a single dose [376-378,380,383] or two or three doses $[364,379,382]$ were given. Although direct comparisons were not made, there was no discernible difference in postoperative SSI rates between single-dose and multidose administration in most studies. A randomized trial specifically comparing different durations of regimens found no statistical difference between a single preoperative dose, three doses (preoperative dose plus two additional doses), or a five-day regimen [386]. A large cohort study found that single doses of metronidazole and gentamicin in patients undergoing open appendectomy were effective and sufficient in decreasing the SSI rate [387].

\section{Pediatric efficacy}

In pediatric patients, as with adults, preoperative determination of complicated versus uncomplicated appendicitis is difficult. A comprehensive review is not provided here, but this topic has been addressed by SIS [388].

Two pediatric studies demonstrated no difference in SSI rates between placebo and several antimicrobials. The first study compared metronidazole, penicillin plus tobramycin, and piperacillin [389]. The second study compared singledose metronidazole and single-dose metronidazole plus cefuroxime [390]. A meta-analysis including both adult and pediatric studies found that for pediatric patients, antimicrobial prophylaxis trended toward being beneficial, but the results were not statistically significant [374]. A retrospective chart review questioned the routine need for antimicrobial prophylaxis in children with simple appendicitis, due to relatively low infection rates in children not receiving prophylaxis [365]. However, these and other study authors have suggested antimicrobial prophylaxis may be considered due to the morbidity associated with infectious complications (e.g., prolonged hospitalization, readmission, reoperation) and due to the inability to preoperatively identify appendicitis.

As a single agent, metronidazole was no more effective than placebo in two double-blind studies that included children 10 years of age or older [360] and 15 years of age or older [363]. In a randomized study that included pediatric patients, ceftizoxime and cefamandole were associated with significantly lower infection rates and duration of hospitalization than placebo [391]. Both cefoxitin and a combination of gentamicin and metronidazole were associated with a lower rate of postoperative infection in a randomized study that included pediatric patients younger than 16 years [378]. Second-generation cephalosporins with anaerobic activity (cefoxitin or cefotetan) and third-generation cephalosporins with anaerobic activity (cefotaxime) were effective, with postoperative infection rates of $<5 \%$ in two studies that included pediatric patients younger than 12 years [364,378,379]. A single dose of gentamicin with clindamycin was found to be safe and effective in children with simple appendicitis [392].

\section{Recommendations}

For uncomplicated appendicitis, the recommended regimen is a single dose of a cephalosporin with anaerobic activity (cefoxitin or cefotetan) or a single dose of a first-generation cephalosporin (cefazolin) plus metronidazole (Table 2). For $\beta$-lactam-allergic patients, alternative regimens include (1) clindamycin plus gentamicin, aztreonam, or a fluoroquinolone and (2) metronidazole plus gentamicin or a fluoroquinolone (ciprofloxacin or levofloxacin). (Strength of evidence for prophylaxis $=$ A.)

\section{Small intestine procedures}

\section{Background}

Small intestine procedures, or small bowel surgery as defined by NHSN, include incision or resection of the small intestine, including enterectomy with or without intestinal anastomosis or enterostomy, intestinal bypass, and strictureoplasty; it does not include small-to-large bowel anastomosis.

The risk of SSI in small bowel surgery is variable. The Surgical Site Infection Surveillance Service in England (data collected by 168 hospitals in 13 categories of surgical procedures between 1997 and 2002) reported an SSI rate of $8.9 \%$ (94 of 1,056) [393]. Mean SSI rates for small bowel procedures reported in the most recent NHSN report (2006-08) were $3.44 \%$ for NHSN risk index category 0 versus $6.75 \%$ for NHSN risk index categories 1, 2, and 3. A study of 1,472 patients undergoing bowel surgery (small bowel and colon) at 31 U.S. 
academic medical centers between September and December 2002 found an SSI rate of $8.7 \%$ for all wound categories. For patients with clean-contaminated wounds, the SSI rate was $7.9 \%$; for those with contaminated or dirty-infected wounds, the SSI rates were $12.0 \%$ and $20.4 \%$, respectively [394].

In a study of 178 penetrating stomach and small bowel injuries, $94 \%$ of which were operated on within six hours of presentation, SSIs occurred in nearly $20 \%$ of cases. When associated colon injuries were excluded, SSIs occurred in $16 \%$ of gastric injuries and 13\% of small bowel injuries. Although $74 \%$ of patients received antimicrobials, the specific timing of antimicrobial administration was not provided [395]. Other studies of small bowel injury confirm similar SSI rates [396-400].

Antimicrobial prophylaxis is recommended for small bowel surgery, based on inferring effectiveness from other clean-contaminated procedures. No specific prospective randomized studies could be identified that addressed antimicrobial prophylaxis for small bowel surgery. Antimicrobial prophylaxis for small bowel surgical procedures related to a diagnosis of complicated intra-abdominal infection is not addressed separately in these guidelines, as antimicrobial therapy for established intraabdominal infection should be initiated preoperatively.

\section{Organisms}

The most common microorganisms isolated from SSIs after small bowel surgery are aerobic gram-negative enteric organisms. Among the species isolated from patients with SSI after small intestine surgery are gram-negative bacilli of gastrointestinal enteric origin (aerobic and anaerobic) and grampositive species, such as streptococci, staphylococci, and enterococci, which is consistent with similar studies [401]. Escherichia coli is the most frequently identified aerobe, indicating that the bowel flora constitute a major source of pathogens. Aerobic and anaerobic streptococci, Staphylococcus species, and Enterococcus species also have been reported.

The microbiology of 2,280 SSIs after upper or lower abdominal surgery conducted from 1999 to 2006 was described in the Prevalence of Infections in Spanish Hospitals (EPINE) study [402]. The most frequent microorganisms isolated were E. coli $(28 \%)$, Enterococcus species $(15 \%)$, Streptococcus species $(8 \%)$, P. aeruginosa (7\%), and S. aureus (5\%; resistant to methicillin, $2 \%$ ). The microbiology of SSIs after upper abdominal tract surgery did not show any significant differences compared with SSIs of the lower tract, though there were relatively more staphylococci, K. pneumoniae, Enterobacter species, Acinetobacter species, and Candida albicans isolates and fewer E. coli, B. fragilis, and Clostridium species in the upper abdominal surgery group [402].

\section{Efficacy}

Antibiotic prophylaxis is generally recognized as effective in the prevention of postoperative SSIs in patients undergoing small bowel surgery when compared with placebo. However, there are no prospective placebo-controlled trials to definitively establish the efficacy of prophylactic antimicrobials in this patient population.

Choice of agent. The antimicrobials selected for prophylaxis must cover the expected pathogens for the small intestine. The microbial ecology of the proximal small intestine (i.e., jejunum) is similar to that of the duodenum, whereas the microbial flora of the ileum are similar to those of the colon. In patients with small intestine obstruction, the microbial flora are similar to those of the colon.

No randomized controlled trials have confirmed that one antimicrobial agent is superior to other agents for SSI prophylaxis in small bowel surgery. An appropriate antimicrobial choice for SSI prophylaxis in small bowel surgery is any single agent or combination of agents that provides adequate coverage for the small intestinal microbes. In patients with small bowel obstruction, additional coverage of anaerobic bacteria is also desirable.

For small intestine procedures with no evidence of obstruction, a first-generation cephalosporin (cefazolin) is recommended. For patients with small intestine obstruction, a first-generation cephalosporin with metronidazole or a second-generation cephalosporin with anaerobic activity (cefoxitin or cefotetan) is the recommended agent. The choice of agents should be based on local drug acquisition costs and antimicrobial sensitivity patterns. The Common Principles section of these guidelines provides additional considerations for weight-based dosing.

Duration. Preoperative dosing of antimicrobials for SSI prevention, with additional intraoperative antimicrobial dosing dependent on the duration of the operation and no postoperative dosing, is recommended for patients undergoing small bowel surgery.

\section{Pediatric efficacy}

In pediatric patients, as with adults, antimicrobial prophylaxis for SSI prevention in small bowel surgery is recommended.

\section{Recommendations}

For small bowel surgery without obstruction, the recommended regimen is a first-generation cephalosporin (cefazolin) (Table 2). For small bowel surgery with intestinal obstruction, the recommended regimen is a cephalosporin with anaerobic activity (cefoxitin or cefotetan) or the combination of a first-generation cephalosporin (cefazolin) plus metronidazole. For $\beta$-lactam-allergic patients, alternative regimens include (1) clindamycin plus gentamicin, aztreonam, or a fluoroquinolone and (2) metronidazole plus gentamicin or a fluoroquinolone (ciprofloxacin or levofloxacin). (Strength of evidence for prophylaxis $=\mathrm{C}$.)

\section{Hernia repair procedures (hernioplasty and herniorrhaphy)}

\section{Background}

All patients who undergo hernioplasty (prosthetic mesh repair of hernia) or herniorrhaphy (suture repair of hernia) should receive appropriate preoperative i.v. antimicrobials for SSI prevention. The risk of SSIs is higher in hernioplasty compared with herniorrhaphy [403]. There is a significant risk of requiring prosthetic mesh removal in hernioplasty patients who develop an SSI, and determination of whether mesh placement will be required for hernia repair is not always possible in the preoperative period.

Mean SSI rates for herniorrhaphy reported in the most recent NHSN report (2006-08) were $0.74 \%$ (21 of 2,852) for 
NHSN risk index category $0,2.42 \%$ (81 of 3,348$)$ for NHSN risk index category 1 , and $5.25 \%(67$ of 1,277$)$ for NHSN risk index categories 2 and 3 [165].

A Cochrane meta-analysis of 17 randomized trials ( $n=7,843 ; 11$ hernioplasty trials, 6 herniorrhaphy trials) in elective open inguinal hernia repair reported SSI rates of 3.1\% versus $4.5 \%$ in the antimicrobial prophylaxis and control groups, respectively (OR, 0.64; 95\% CI, 0.50-0.82) [404]. The subgroup of patients with herniorrhaphy had SSI rates of $3.5 \%$ and $4.9 \%$ in the prophylaxis and control groups, respectively (OR, $0.71 ; 95 \% \mathrm{CI}, 0.51-1.00)$. The subgroup of patients with hernioplasty had SSI rates of $2.4 \%$ and $4.2 \%$ in the prophylaxis and control groups, respectively (OR, 0.56; 95\% CI, 0.38-0.81).

A meta-analysis of nine randomized trials of open hernioplasty for inguinal hernia documented SSI rates of $2.4 \%$ (39 of $1,642)$ in the antimicrobial group and $4.2 \%(70$ of 1,676$)$ in the control group. Antibiotics showed a protective effect in preventing SSI after mesh inguinal hernia repair (OR, $0.61 ; 95 \%$ CI, 0.40-0.92). Antibiotic prophylaxis did reduce the rate of SSI in hernia patients undergoing mesh hernioplasty [405].

Based on the results of these two systematic reviews, preoperative antimicrobial prophylaxis for SSI prevention is recommended for both herniorrhaphy and hernioplasty. Compared with open hernia repair, laparoscopic hernia repair has been reported to produce lower rates of incisional (superficial and deep) SSIs in randomized clinical trials [406408]. In a recent multicenter randomized trial of laparoscopic versus open ventral incisional hernia repair $(n=162)$, SSI was significantly less common in the laparoscopic group than in the open repair group $(2.8 \%$ versus $21.9 \%$; OR, $10.5 ; 95 \% \mathrm{CI}$, 2.3-48.2; $p=0.003$ ) [409]. A meta-analysis of eight randomized trials comparing laparoscopic and open incisional or ventral hernia repair with mesh revealed that laparoscopic hernia repair was associated with decreased SSI rates (relative risk, 0.22 ; 95\% CI, 0.09-0.54) and a trend toward fewer infections requiring mesh removal [410].

\section{Organisms}

The most common microorganisms isolated from SSIs after herniorrhaphy and hernioplasty are aerobic gram-positive organisms. Aerobic streptococci, Staphylococcus species, and Enterococcus species are common, and MRSA is commonly found in prosthetic mesh infections [411].

\section{Efficacy}

Antibiotic prophylaxis is generally recognized as effective when compared with placebo in the prevention of postoperative SSIs in patients undergoing herniorrhaphy and hernioplasty.

Choice of agent. Randomized controlled trials have failed to identify an agent that is clearly superior to other agents for SSI prophylaxis in hernia repair. A first-generation cephalosporin is the recommended agent on the basis of cost and tolerability. The Common Principles section of these guidelines provides additional considerations for weight-based dosing.

Duration. Based on the evidence to date, a single preoperative dose of antimicrobial is recommended in hernioplasty and herniorrhaphy, with redosing as recommended in the
Common Principles section of these guidelines (if the procedure duration exceeds the recommended redosing interval from the time of initiation of the preoperative dose or if there is prolonged or excessive bleeding).

\section{Recommendations}

For hernioplasty and herniorrhaphy, the recommended regimen is a single dose of a first-generation cephalosporin (cefazolin) (Table 2). For patients known to be colonized with MRSA, it is reasonable to add a single preoperative dose of vancomycin to the recommended agent. For $\beta$-lactam-allergic patients, alternative regimens include clindamycin and vancomycin. (Strength of evidence for prophylaxis = A.)

\section{Colorectal procedures}

\section{Background}

Surgical site infections have been reported to occur in approximately $4-10 \%$ of patients undergoing colon procedures, $3-7 \%$ in small bowel procedures, and $3-27 \%$ in patients after rectal procedures, based on the risk index [165]. However, when patients are followed carefully in clinical trials, rates tend to be considerably higher (17-26\%) [412]. Other septic complications, such as fecal fistula, intra-abdominal abscesses, peritonitis, and septicemia, are serious concerns but are much less common [413]. Infectious complication rates range from $30 \%$ to $60 \%$ without antimicrobial prophylaxis $[59,414]$ and are $<10 \%$ with appropriate antimicrobial prophylaxis. A pooled analysis of clinical trials of antimicrobial prophylaxis in colon procedures demonstrated that antimicrobial use significantly reduced mortality rates $(11.2 \%$ for control versus $4.5 \%$ for treatment) and SSI rates [415].

The type and duration of the procedure can affect the risk of infection. Rectal resection is associated with a higher risk of infection than is intraperitoneal colon resection [416-418]. Other risk factors include extended procedure duration (e.g., $>3.5$ hours) [59,412,418,419], impaired host defenses [418], age of $>60$ years [418], hypoalbuminemia [419,420], bacterial or fecal contamination of the surgical site $[418,420]$, inadvertent perforation or spillage [412,421], corticosteroid therapy [419], perioperative transfusion of packed red blood cells [394,418], hypothermia [422], hyperglycemia [423,424], and obesity $[412,418]$.

\section{Organisms}

The infecting organisms in colorectal procedures are derived from the bowel lumen, where there are high concentrations of organisms. Bacteroides fragilis and other obligate anaerobes are the most frequently isolated organisms from the bowel, with concentrations 1,000-10,000 times higher than those of aerobes [425]. Escherichia coli is the most common aerobe. Bacteriodes fragilis and Escherichia coli comprise approximately $20-30 \%$ of the fecal mass. They are the most frequently isolated pathogens from infected surgical sites after colon procedures.

\section{Efficacy}

Results from randomized controlled trials and a Cochrane review of 182 studies of over 30,000 patients support the routine use of prophylactic antimicrobials in all patients undergoing colorectal procedures [426]. 
Choice of agent. The agent chosen for antimicrobial prophylaxis in colorectal procedures should have activity against the anaerobic and aerobic floras of the bowel. The most appropriate regimen for antimicrobial prophylaxis for colorectal procedure (e.g., oral, i.v, oral-i.v. combination) and the optimal choice of antimicrobial agent have not been fully resolved.

Oral regimens. The efficacy of oral prophylactic antimicrobial agents has been established in studies only when used with mechanical bowel preparation (MBP). A variety of oral agents administered after MBP have been evaluated for prophylaxis for colorectal procedures. The most common combinations include an aminoglycoside (neomycin and, less often, kanamycin, which is only available in injectable form in the United States) plus a medication with anaerobic activity, usually erythromycin [427-434] or metronidazole [432,433,435-439]. In placebo-controlled studies, the oral combination was significantly more effective than placebo in reducing SSIs [427,433,434,439,440]. Postoperative SSI rates were $0-11 \%$ with neomycin plus erythromycin [427-432] and $2-13 \%$ with neomycin and metronidazole [436-438]. Combinations of neomycin and tetracycline [440], neomycin and clindamycin [436], and neomycin and tinidazole [441] have also been used successfully, with postoperative SSI rates of $<10 \%$. The use of metronidazole as a single agent appears to be less effective, with reported SSI rates of 12-15\% [442-444].

Oral antimicrobials have been compared with i.v. agents in a few studies. Oral neomycin plus oral erythromycin was similarly effective as i.v. cefoxitin in one study [429] but inferior in another [445] and was similarly effective as i.v. ceftriaxone plus i.v. metronidazole in patients undergoing elective colorectal procedures [431]. The addition of i.v. cefamandole to oral neomycin plus oral erythromycin did not improve efficacy [430]. In one of these studies, oral neomycin and erythromycin were more effective than i.v. cefoxitin for

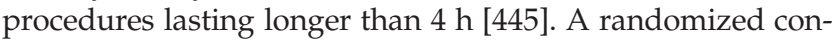
trolled study was stopped early due to the significantly higher rate of infection in the oral neomycin and erythromycin group $(41 \%)$ compared with the single-dose i.v. metronidazole and ceftriaxone group $(9.6 \%)(p<0.01)$ [446]. Similarly, a study of oral metronidazole and kanamycin compared with the same medications given intravenously found an increased rate of postoperative sepsis ( $36 \%$ versus $6.5 \%$, respectively) $(\mathrm{p}<0.001)$, greater numbers of E. coli resistant to kanamycin, more bacterial overgrowth, and antimicrobial-associated pseudomembranous colitis in the oral group [447]. However, the oral antimicrobials were not given on a schedule expected to be effective, as they were discontinued $36 \mathrm{~h}$ before the procedure. The fact that oral antibiotics were given for three days rather than less than one day, as is the current practice, was suggested as a possible reason for the resistance and colitis observed.

I.V. regimens. A wide range of i.v. antimicrobials have been evaluated for prophylaxis in colorectal procedures. Cephalosporins are the most common agents, usually administered as a single agent. The majority of studies found that single-agent firstgeneration cephalosporins (cefazolin and cephalothin) [445,448451] were ineffective, with postoperative SSI rates ranging from $12 \%$ to $39 \%$ [448,449]. The lack of efficacy is likely due to their lack of $B$. fragilis activity. The combination of cefazolin and metronidazole provides adequate coverage of pathogens and may be a cost-effective prophylaxis strategy [6,41].
Second-generation cephalosporins with anaerobic activity, such as cefoxitin and cefotetan, have been widely evaluated. In single-agent therapy, SSI rates ranged from $0 \%$ to $17 \%$ $[91,417,445,452-459]$; however, more than half of the studies found SSI rates of $>10 \%$.

Third-generation agents, cefotaxime and ceftriaxone, have been evaluated in a few trials; postoperative SSI rates were $8-19 \%$ with single-agent use $[456,460,461]$. In some studies, second- or third-generation cephalosporins were combined with other i.v. agents, most commonly metronidazole [452,459462]. However, in all but one of these studies, a combination of a second- or third-generation cephalosporin plus metronidazole was no more effective than the cephalosporin alone. The use of third- or fourth-generation cephalosporins for routine antimicrobial prophylaxis is not recommended as use may lead to development of resistant organisms [6,41,444,463]. However, in institutions where there is increasing gram-negative resistance from isolates to first- and second-generation cephalosporins, a single dose of ceftriaxone plus metronidazole may be preferred over routine use of a carbapenem.

Three small studies, with under 200 patients each, found i.v. ampicillin-sulbactam or amoxicillin-clavulanic acid to be as effective as i.v. combinations of gentamicin and metronidazole [464], gentamicin and clindamycin [465], and cefotaxime and metronidazole for preventing SSIs in elective colorectal procedures.

A randomized controlled study of adult patients undergoing elective colon or rectal procedures evaluated the use of a single high dose of gentamicin $4.5 \mathrm{mg} / \mathrm{kg}$ i.v. plus metronidazole $500 \mathrm{mg}$ i.v. in sequential order over $30 \mathrm{~min}$ compared with multiple standard doses of gentamicin $1.5 \mathrm{mg} / \mathrm{kg}$ plus metronidazole given preoperatively and every $8 \mathrm{~h}$ for $24 \mathrm{~h}$ postoperatively [16]. All patients underwent MBP before surgery. Patients with a serum creatinine concentration exceeding $1.7 \mathrm{mg} / \mathrm{dL}$ were excluded from the study. No statistically significant differences were seen in deep and superficial incisional SSI rates between groups. Significantly fewer superficial SSIs were seen in the single-dose group compared with the multidose group in procedures lasting longer than $3.5 \mathrm{~h}(22.2 \%$ versus $55 \%, p=0.021$ ). A pharmacodynamic study of these patients found the gentamicin concentration at the time of surgical site closure as the strongest independent factor for infection [17]. Of note, the infection rate was $80 \%$ in 10 patients with gentamicin concentrations of $<0.5 \mathrm{mg} / \mathrm{L}$.

Other i.v. agents that have been evaluated either alone or in combination include aminoglycosides [464,466-469], clindamycin [466], ampicillin [467,469-471], penicillins plus $\beta$-lactamase inhibitors [464,465,468,472,473], doxycycline [470,474-476], piperacillin [91,473], imipenem-cilastatin [462], and ciprofloxacin [300].

Ertapenem, a broad-spectrum carbapenem, is approved by FDA for the prophylaxis of SSIs after elective colorectal procedures [67]. Cefotetan is also FDA-approved for surgical prophylaxis in clean-contaminated procedures (e.g., gastrointestinal procedures) in adult patients undergoing elective colon or rectal procedures [62]. A large, multicenter, randomized controlled study compared a single $1 \mathrm{~g}$ i.v. dose of ertapenem with cefotetan $2 \mathrm{~g}$ i.v. infused within $60 \mathrm{~min}$ before surgical incision [412]. All patients received MBP preoperatively. Rates of SSI were significantly lower in the ertapenem group versus cefotetan in the per-protocol (18.1\% and $31.1 \%$, respectively) and the modified intent-to-treat (17.1\% and 50.9\%) populations. Ertapenem was found to be superior to cefotetan for SSI 
prevention. Although not statistically significant, higher rates of skin-related events (i.e., pruritis and rash), gastrointestinal events, and $C$. difficile infection were seen in the ertapenem group. The study authors concluded that ertapenem is an acceptable alternative to cefotetan and cefoxitin. Routine use of ertapenem for surgical prophylaxis remains controversial due to theoretical concerns regarding increases in resistant organisms and a potential increase in adverse events [477].

Alternative agents for patients with a high likelihood of past serious adverse event or allergy to $\beta$-lactams include (1) clindamycin plus an aminoglycoside, aztreonam, or a fluoroquinolone and (2) metronidazole plus an aminoglycoside or a fluoroquinolone [41].

Combination oral and i.v. regimens. Combinations of oral and i.v. antimicrobials have been used in an attempt to further reduce postoperative infection rates. Regimens include oral neomycin and erythromycin plus i.v. administration of a cephalosporin [416,417,429,445,449,478,479], metronidazole [480,481], and gentamicin plus clindamycin [466]. Postoperative SSI rates in these studies ranged from $0 \%$ to $7 \%$. With one exception [416], there was no significant difference between oral neomycin-erythromycin plus an i.v. antimicrobial and oral neomycin-erythromycin alone $[429,449,466,478]$. When combination oral and i.v. agents were compared with i.v. agents alone, combination therapy was favored in five of six studies $[417,429,445,449,480,482]$; the difference was significant in three $[417,449,482]$. The most recent Cochrane review found that the infection rate was significantly lower with the combination of oral plus i.v. prophylaxis when compared with i.v. alone (relative risk, $0.55 ; \mathrm{p}=0.000084$ ) or with oral prophylaxis alone (relative risk, $0.34 ; \mathrm{p}=0.024$ ) [426]. A recent report of over 2,000 patients recorded prospectively in the Michigan Surgical Quality Collaborative-Colectomy Best Practices Project and analyzed retrospectively revealed a significantly lower rate of postoperative infections when 370 colectomy patients received MBP and oral antimicrobial prophylaxis compared with propensity-matched patients receiving i.v. prophylaxis alone [483].

A multicenter, randomized, controlled study of 491 patients who received MBP plus oral antimicrobials (kanamycin and erythromycin) with i.v. cefmetazole (not available in the United States but noted by the expert panel to have a similar spectrum of activity as cefotetan) or i.v. cefmetazole alone found no difference in SSI between groups for colon procedures [484]. However, the combination of oral and i.v. antimicrobials was significantly better than i.v. alone for rectal procedures, particularly abdominoperineal excision. Another study found the postoperative SSI rates after rectal resection were $23 \%$ and $11 \%$, respectively, for patients receiving i.v. cefoxitin and cefoxitin plus oral neomycin and erythromycin [417].

The safety and tolerability of oral antimicrobials have been investigated in two studies. One case-control study found an increased incidence of $C$. difficile colitis among patients with oral plus i.v. antimicrobials and MBP compared with i.v. antimicrobials and MBP alone [485]. However, another casecontrol study found a lower rate (not statistically significant) of $C$. difficile infection in patients who had received oral antimicrobials compared with those who had not $(1.6 \%$ versus $2.9 \%, p=0.09$ ) [486]. A randomized controlled study of 300 patients undergoing elective colorectal procedures found significantly higher rates of nausea and vomiting among patients receiving three doses of oral antimicrobials (neomycin and metronidazole, $44 \%$ and $31 \%$, respectively) in combination with i.v. cefoxitin and MBP compared with regimens including one dose of oral antimicrobials (18\% and $11 \%$, respectively) and no oral antimicrobials (13\% and $9 \%$, respectively) [487]. No difference was noted between groups for rates of abdominal pain, SSIs, or intraabdominal abscesses. An increased number of gastrointestinal adverse events was also reported in another comparative study in the combination oral and i.v. group $(2.9 \%)$ compared with the i.v.-only group (2.1\%), although the results were not statistically significant [484]. Overall, the evidence suggests that the combination of oral antimicrobials with MBP in addition to i.v. prophylactic antimicrobials reduces the rate of postoperative infections compared with i.v. antimicrobials alone without MBP, although the addition of oral antimicrobials increases gastrointestinal symptoms.

Duration. Single and multiple doses were compared in several studies $[454-456,461,471,475]$. However, only two of these studies compared single doses with multiple doses of the same antimicrobial $[471,475]$. There was no significant difference in infection rates between single-dose and multidose administration. One study found a single dose of cefotaxime plus metronidazole was significantly more effective than three doses of cefotaxime alone [461]. The most recent Cochrane review found no benefit to extending the duration of prophylaxis $(p=0.58)$ [426]. Generally, antimicrobial prophylaxis should be continued for no more than $24 \mathrm{~h}$ and can typically be stopped when the procedure is completed and the surgical site is closed $[6,41,444]$. No evidence supports greater efficacy for doses given after the completion of the procedure. Additional discussion on this topic is found in the Common Principles section of these guidelines.

Consideration should be given to an additional dose of the i.v. antimicrobial if an agent with a short half-life is used and the procedure duration exceeds the recommended redosing interval (starting from the time of initiation of the preoperative dose) and if intraoperative blood loss occurs [6,41,120,418, $444,445]$. No significant difference was seen in SSI rates with single-dose cefazolin, single-dose cefotetan, and cefazolin given as one preoperative dose and a second dose three hours later for procedures with a duration of less than three hours [118]. Rates of SSI were significantly higher with a single dose of cefazolin for procedures with a duration of greater than three hours. Using an agent with a longer half-life can decrease the necessity to redose the antimicrobial during long procedures.

\section{Pediatric efficacy}

No well-controlled studies have evaluated the efficacy of antimicrobial prophylaxis in pediatric patients undergoing colorectal procedures. However, there is no reason to suspect that prophylaxis efficacy would be different. The safety, efficacy, tolerability, and cost-effectiveness of intestinal lavage have been demonstrated in two studies of 20 and 21 pediatric patients $[488,489]$.

\section{Recommendations}

A single dose of second-generation cephalosporin with both aerobic and anaerobic activities (cefoxitin or cefotetan) or cefazolin plus metronidazole is recommended for colon procedures (Table 2). In institutions where there is increasing resistance to first- and second-generation cephalosporins 
among gram-negative isolates from SSIs, the expert panel recommends a single dose of ceftriaxone plus metronidazole over routine use of a carbapenem. An alternative regimen is ampicillin-sulbactam. In most patients, MBP combined with a combination of oral neomycin sulfate plus oral erythromycin base or oral neomycin sulfate plus oral metronidazole should be given in addition to i.v. prophylaxis. The oral antimicrobial should be given as three doses over approximately 10 hours the afternoon and evening before the operation and after the MBP. Alternative regimens for patients with $\beta$-lactam allergies include (1) clindamycin plus an aminoglycoside, aztreonam, or a fluoroquinolone and (2) metronidazole plus an aminoglycoside or a fluoroquinolone. Metronidazole plus aztreonam is not recommended as an alternative because this combination has no aerobic gram-positive activity [385]. (Strength of evidence for prophylaxis $=$ A.)

\section{Head and neck procedures}

\section{Background}

Elective procedures of the head and neck are predominantly clean or clean-contaminated [490]. Clean procedures include thyroidectomy and lymph node excisions. Cleancontaminated procedures include all procedures involving an incision through the oral or pharyngeal mucosa, ranging from parotidectomy, submandibular gland excision, tonsillectomy, adenoidectomy, and rhinoplasty to complicated tumordebulking and mandibular fracture repair procedures requiring reconstruction. The frequency of SSIs reported for clean procedures without antimicrobial prophylaxis is $<1 \%$ [491,492]. In contrast, infection rates in patients undergoing complicated head and neck cancer surgery are quite high, with infection occurring in $24-87 \%$ of patients without antimicrobial prophylaxis [493-497]. While many of these head and neck cancer procedures are clean-contaminated, these procedures can fall into different wound classifications. Head and neck cancer patients often have many of the risk factors for infection mentioned below [498].

Postoperative SSI rates are affected by age, nutritional status, and the presence of concomitant medical conditions such as diabetes mellitus, anemia, and peripheral vascular disease [496,499-504]. Use of tobacco [498,505], alcohol [505,506], or drugs of abuse [507] has also been associated with a higher risk of postoperative infection, particularly in patients with mandibular fracture. The hospital course, including length of hospitalization before operation, duration of antimicrobial use before operation, length of operation, presence of implants, and previous tracheotomy can also affect postoperative SSI rates [496,497,501-504,508]. In patients with cancer, preoperative radiation and chemotherapy as well as the stage of the malignancy may also affect infection risk [497,498,502504]. Procedure-related risk factors for infection include radical or bilateral neck dissections [501,508] and reconstruction with myocutaneous flaps or microvascular-free flaps [497-499,508].

\section{Organisms}

The normal floras of the mouth and the oropharynx are responsible for most infections that follow clean-contaminated head and neck procedures [6,8,496,498,499,506,509-519]. Anaerobic and aerobic bacteria are abundant in the oropharynx. As a result, postoperative SSIs are usually polymicrobial and involve both aerobic and anaerobic bacteria. The predominant oropharyngeal organisms include various streptococci (aerobic and anaerobic species), other oral anaerobes including Bacteroides species (but not B. fragilis), Peptostreptococcus species, Prevotella species, Fusobacterium species, Veillonella species, Enterobacteriaceae, and staphylococci. Nasal flora includes Staphylococcus species and Streptococcus species.

\section{Efficacy}

Clean procedures. Systemic administration of prophylactic antimicrobials has not been proven effective in reducing SSI rates in patients undergoing clean procedures of the head and neck and are not recommended for routine use [6-8, $497,520]$. One randomized, double-blind, multicenter study of 500 patients undergoing thyroid procedures for goiter or carcinoma found no difference in postoperative SSI rates in those who received antimicrobial prophylaxis $(0.8 \%)$ and those who did not $(0.4 \%)$ [491].

Clean-contaminated procedures. Based on the best available evidence, current guidelines and review articles recommend the use of antimicrobial prophylaxis for the majority of clean-contaminated procedures [6-8,497,520,521]. However, antimicrobial prophylaxis did not lower infection risk in randomized controlled trials of patients undergoing adenoidectomy, tonsillectomy [522,523], and septoplasty [524], and systematic reviews have not recommended prophylaxis for these procedures $[7,525,526]$.

The efficacy of antimicrobial prophylaxis is best established for head and neck cancer surgery. Several small randomized, controlled trials found high infection rates in placebo groups (24-78\%) and markedly lower infection rates in the prophylaxis groups (5.8-38\%) using a variety of regimens, including cefazolin, third-generation cephalosporins, and ampicillin plus cloxacillin. Although these studies were small, the results are concordant, and the high infection rates allowed the studies to reach statistical significance despite the small sample sizes. Similar results were reported in several additional small, uncontrolled studies [500,527-529].

Choice of agent. Several randomized, single-center studies have compared antimicrobial regimens for cleancontaminated procedures. In one study, 189 patients undergoing head and neck cancer procedures were randomized to receive cefazolin $1 \mathrm{~g}(n=92)$ or amoxicillin-clavulanic acid ( $n=97)$, both given within one hour of incision and every eight hours postoperatively for three doses [511]. The postoperative SSI rates were $24 \%$ with cefazolin and $21 \%$ with amoxicillinclavulanic acid; there was no statistically significant difference in infection rates in this underpowered study. Two studies have compared ampicillin-sulbactam to clindamycin and yielded discordant results. One study of 242 patients (169 evaluable) undergoing head and neck cancer procedures compared ampicillin-sulbactam $1.5 \mathrm{~g}(n=119)$ and clindamycin $600 \mathrm{mg}$ $(n=123)$ given within one to two hours of incision and every six hours postoperatively for a total of four doses [510]. No difference in SSIs was found, with 15 infections reported in each group (13\% for the ampicillin-sulbactam group and $12 \%$ for the clindamycin group). There was no significant difference in adverse events between groups. There was a higher rate of $C$. difficile-positive patients in the clindamycin group 
$(n=7)$ than in the ampicillin-sulbactam group $(n=1)$, with no reported statistical analysis. Another study of 212 patients undergoing clean-contaminated head and neck oncology surgery found significantly fewer infections in the ampicillin-sulbactam group $(13.3 \%)$ compared with the clindamycin group (27.1\%) $(p=0.02)$ [530]. A greater number of gram-negative pathogens were recovered from patients randomized to the clindamycin group. The combination of gentamicin and clindamycin was superior to cefazolin in one older clinical trial [531].

Duration. Studies of clean-contaminated head and neck procedures found no difference in efficacy between regimens of 24 hours and longer regimens of three, five, or seven days [499-501,505,507,512,524,531-534]. Limited data exist on single-dose prophylaxis in these procedures.

One study of patients undergoing free-flap reconstruction after head and neck procedures found a significantly lower rate of acquisition and infection with MRSA in patients receiving short-term cefuroxime and metronidazole (one dose during induction of anesthesia and one dose eight hours postoperatively) compared with long-term therapy (same antimicrobials with additional doses every eight hours for up to five days) ( $p=0.005$ and $p=0.01$, respectively, for acquisition and infection) [535].

\section{Recommendations}

Clean procedures. Antimicrobial prophylaxis is not required in patients undergoing clean surgical procedures of the head and neck. If there is placement of prosthetic material, a preoperative dose of cefazolin or cefuroxime is reasonable, though there are few data supporting the efficacy of prophylaxis in this setting (Table 2). A reasonable alternative for patients with $\beta$-lactam allergies is clindamycin. (Strength of evidence against prophylaxis without prosthesis placement $=\mathrm{B}$; strength of evidence for prophylaxis with prosthesis placement $=\mathrm{C}$.)

Clean-contaminated procedures. Antimicrobial prophylaxis has not been shown to benefit patients undergoing tonsillectomy or functional endoscopic sinus procedures. The preferred regimens for patients undergoing other cleancontaminated head and neck procedures are (1) cefazolin or cefuroxime plus metronidazole and (2) ampicillin-sulbactam. Clindamycin is a reasonable alternative in patients with a documented $\beta$-lactam allergy. The addition of an aminoglycoside to clindamycin may be appropriate when there is an increased likelihood of gram-negative contamination of the surgical site. (Strength of evidence for prophylaxis in cancer surgery patients $=\mathrm{A}$; strength of evidence for prophylaxis for other clean-contaminated procedures except tonsillectomy and functional endoscopic sinus procedures $=\mathrm{B}$.)

\section{Neurosurgery procedures}

\section{Background}

Nosocomial central nervous system (CNS) infections do not often occur but have potentially serious consequences and poor outcomes, including death [536]. One of the greatest risks for these infections in children and adults is undergoing a neurosurgical procedure. A classification system for neurosurgery, validated by Narotam et al. [537], divides procedures into five categories: clean, clean with foreign body, clean-contaminated, contaminated, and dirty. Risk factors for postoperative infections after neurological procedures include an ASA classification of $\geq 2$ [538], postoperative monitoring of intracranial pressure $[538,539]$ or ventricular drains $[536,538]$ for five or more days, cerebrospinal fluid (CSF) leak [539-541], procedure duration of more than two to four hours [540,542-544], diabetes [544], placement of foreign body [536], repeat or additional neurosurgical procedures [538,541-543], concurrent (remote, incision, or shunt) or previous shunt infection $[536,539,545,546]$, and emergency procedures $[542,545]$.

\section{Organisms}

Data from most published clinical trials indicate that SSIs are primarily associated with gram-positive bacteria, S. aureus, and coagulase-negative staphylococci $[6,8,537-545,547-554]$. Several cohort studies revealed high rates (up to $75-80 \%$ of isolates) of MRSA [540-543,548-552] and coagulase-negative staphylococci among patients undergoing a variety of neurosurgical procedures $[539,540,543,549]$. Other skin organisms such as $P$. acnes may be seen after CSF shunt placement, craniotomy, and other procedures $[536,555,556]$. Gram-negative bacteria have also been isolated as the sole cause of postoperative neurosurgical SSIs in approximately $5-8 \%$ of cases and have been isolated in polymicrobial infections [537-539,541-545,547-550,552,553].

\section{Efficacy}

Clean procedures. Antimicrobial prophylaxis is recommended for adult and pediatric patients undergoing craniotomy and spinal procedures $[7,520]$. One meta-analysis of six studies found decreased odds of meningitis in patients undergoing craniotomy who received antimicrobial prophylaxis $(1.1 \%)$ versus no prophylaxis $(2.7 \%)(p=0.03)$ [557]. Two cohort studies [540,543] in patients undergoing craniotomy at the same institution found that antimicrobial prophylaxis with cloxacillin or amoxicillin-clavulanic acid, clindamycin for $\beta$-lactam-allergic patients, and other antimicrobials (not detailed) had a significantly lower infection rate $(5.8 \%)$ than no prophylaxis $(9.7 \%)(p<0.0001)$ [543]. A significantly lower infection rate of $4.6 \%$ was seen in low-risk patients (clean craniotomy, no implant) with antimicrobial prophylaxis compared with those without prophylaxis $(4.6 \%$ versus $10 \%$, $\mathrm{p}<0.0001)$. A significantly lower rate of scalp infections, bone flap osteitis, and abscess or empyema was seen with antimicrobial prophylaxis compared with no prophylaxis. Antimicrobial prophylaxis demonstrated no difference in postoperative meningitis $[540,543]$ and infection rates in highrisk patients (those undergoing emergency, clean-contaminated, and dirty procedures or reoperation or with operative times exceeding four hours) [543].

Prospective studies involving large numbers of patients have also demonstrated lower neurosurgical postoperative infection rates when antimicrobial prophylaxis is used [558561]. One such study of patients undergoing craniotomy, spinal, or shunting procedures was stopped early because of an excessive number of SSIs in the placebo group [562].

Choice of agent. Studies of clean neurosurgical procedures reported antimicrobial regimens including clindamycin [540,543,557], vancomycin [542,557], cefotiam (not marketed in the United States) [557], piperacillin [557], cloxacillin [540,543,557], oxacillin [542,557], cefuroxime [547], 
cefotaxime [548], sulfamethoxazole-trimethoprim [548], cefazolin [542,544], penicillin G [542], and amoxicillin-clavulanic acid [540,542,543]. A meta-analysis found no significant difference in the rates of postcraniotomy meningitis with various antimicrobial regimens (single-dose regimens of clindamycin, vancomycin, or cefotiam; three doses of piperacillin; four doses of cloxacillin; and six doses of oxacillin) [557].

A randomized, open-label, multicenter study of 613 adult patients undergoing elective craniotomy, shunt, or stereotactic procedures found no difference in single doses of cefotaxime and trimethoprim-sulfamethoxazole in postoperative abscess formation, SSIs, and shunt infections [548].

Duration. The majority of studies included single doses of antimicrobials; therefore, the use of single-dose antimicrobial prophylaxis given within $60 \mathrm{~min}$ before surgical incision in patients undergoing neurosurgery is generally recommened $[6,7,520,540,543,547,548,557,563]$.

\section{Efficacy for CSF shunting procedures}

Antimicrobial prophylaxis is recommended for adults undergoing placement of a CSF shunt [7]. Prophylaxis in patients undergoing ventriculostomy or intraventricular prophylaxis at the time of ventriculoperitoneal shunt insertion has shown some benefit in reducing infection but remains controversial due to limited evidence [6,7].

Because CNS infections after shunting procedures are responsible for substantial mortality and morbidity, especially in children, the possible role of prophylactic antimicrobials in such procedures has been studied in numerous small, wellconducted, randomized controlled trials [564-571]. Meticulous surgical and aseptic techniques and short procedure times were determined to be important factors in lowering infection rates after shunt placement. Although the number of patients studied in each trial was small, two meta-analyses of these data demonstrated that antimicrobial prophylaxis use in CSF-shunting procedures reduced the risk of infection by approximately $50 \%$ [572,573].

Intrathecal pump placement involves the implantation of a permanent intrathecal catheter to allow instillation of medication. Central nervous system infections may occur after these procedures, which are performed in both pediatric and adult populations. Several retrospective series have reported infection rates of $4.5-9 \%$ after intrathecal baclofen pump placement [574-576]. There are minimal published trial data regarding appropriate prophylaxis for intrathecal pump procedures. It has been suggested that prophylaxis for intrathecal pump procedures be managed similarly to prophylaxis for CSF shunting procedures [577].

There is no consensus on the use of antimicrobial prophylaxis in patients with extraventricular drains (EVDs) or intracranial pressure monitors [134]. An international survey of neurosurgeons and critical care medicine and infectious diseases specialists illustrates the difference in practices. The majority of neurosurgeons used or recommended the use of antimicrobial prophylaxis with EVDs $(73.5 \%)$ and other monitoring devices (59\%), compared with rates of $46-59 \%$ for critical care medicine specialists and $35 \%$ for infectious diseases specialists. The majority of specialists did not recommend or use antimicrobial-coated EVD catheters.

Two randomized controlled studies comparing antimicrobialimpregnated shunts to standard, non-antimicrobial- impregnated shunts along with antimicrobial prophylaxis with i.v. cephalosporin found a decrease in rates of shunt infections [549] and a significant decrease in CSF infection with antimicrobial-impregnated shunts [545]. At this time, routine use of antimicrobial-impregnated devices is not recommended; additional well-designed studies are needed to establish their place in therapy $[7,578]$.

Choice of agent. In CSF-shunting procedures, no single antimicrobial agent has been demonstrated to have greater efficacy than others $[546,548,551-554,579]$. There is a lack of data on the necessity of antimicrobials with CNS penetration relating to prevention of infection in CNS shunting procedures.

Duration. The majority of studies support the use of single-dose prophylaxis regimens or regimens with a duration of 24-48 hours postoperatively [6-8,520,539,546,549$552,579]$. There is a lack of data evaluating the continuation of EVDs with and without antimicrobial prophylaxis. The international survey mentioned above asked respondents to indicate their recommended duration for antimicrobial prophylaxis with EVDs as either periprocedural, for 24 hours, for the first three days, for the entire time the device is in place, or other [135]. The respondents from the specialties of neurosurgery, neurocritical care, and critical care had similar results, with $28-31 \%$ using or recommending periprocedural antimicrobials, $4-10 \%$ for 24 hours, $2-4 \%$ for the first three days, $43-64 \%$ for the entire time the device is in place, and 0$14 \%$ for other. The infectious diseases specialists reported rates of $62 \%, 19 \%, 4 \%, 12 \%$, and $4 \%$, respectively.

One retrospective single-center cohort study of 308 patients with EVDs placed for three days or more received antimicrobial prophylaxis for the duration of EVD use $(n=209)$ compared with patients receiving cefuroxime $1.5 \mathrm{~g}$ i.v. every eight hours for three doses or less frequently periprocedurally (timing not clearly defined in article) $(n=99)$ [580]. The overall rate of bacterial ventriculitis was $3.9 \%$, with 8 patients $(3.8 \%)$ in the extended-use group and 4 patients (4\%) in the short-term prophylaxis group, the difference of which was not significant. The study authors concluded that there was no benefit to the use of a prolonged duration of antimicrobial prophylaxis.

\section{Pediatric efficacy for CSF shunting procedures}

Antimicrobial prophylaxis is recommended for children undergoing a CSF-shunting procedure [7]. The efficacy of antimicrobial prophylaxis is extrapolated from adult studies.

A retrospective pediatric study of 384 CSF shunting procedures found a lower infection rate in patients who received antimicrobials (2.1\%) compared with those who did not $(5.6 \%)$, but this difference failed to reach statistical significance [581]. Two randomized, prospective studies that included pediatric patients did not demonstrate a significant difference in infection rates between the control group and the groups that received cefotiam [571] (not available in the United States) or methicillin [568]. A randomized, double-blind, placebo-controlled study that included pediatric patients undergoing ventriculoperitoneal shunt surgeries failed to demonstrate that the use of perioperative sulfamethoxazole-trimethoprim reduced the frequency of shunt infection [564].

Other studies have demonstrated efficacy for prophylactic antimicrobials [566,582]. A single-center, randomized, double- 
blind, placebo-controlled trial of perioperative rifampin plus trimethoprim was performed in pediatric patients [582]. Among patients receiving rifampin plus trimethoprim, the infection rate was $12 \%$, compared with $19 \%$ in patients receiving placebo. The study was ended because of the high infection rates before significance could be achieved. Infection rates at the study institution had been $7.5 \%$ in the years before the study. An open-label randomized study, including pediatric patients, demonstrated a lower infection rate in a group receiving oxacillin (3.3\%) than in a control group (20\%) [566].

\section{Recommendations}

A single dose of cefazolin is recommended for patients undergoing clean neurosurgical procedures, CSF-shunting procedures, or intrathecal pump placement (Table 2). Clindamycin or vancomycin should be reserved as an alternative agent for patients with a documented $\beta$-lactam allergy (vancomycin for MRSA-colonized patients). (Strength of evidence for prophylaxis $=$ A.)

\section{Cesarean delivery procedures}

\section{Background}

Approximately 1.2 million infants are born by cesarean delivery in the United States annually [583]. The infection rate after cesarean delivery has been reported to be 4-15\% [583], though recent NHSN data showed an infection rate of 2-4\% [165].

Postpartum infectious complications are common after cesarean delivery. Endometritis (infection of the uterine lining) is usually identified by fever, malaise, tachycardia, abdominal pain, uterine tenderness, and sometimes abnormal or foul-smelling lochia [584]. Fever may also be the only symptom of endometritis.

Endometritis has been reported to occur in up to $24 \%$ of patients in elective cesarean delivery and up to approximately $60 \%$ of patients undergoing nonelective or emergency section [584,585]. Risk factors for endometritis include cesarean delivery, prolonged rupture of membranes, prolonged labor with multiple vaginal examinations, intrapartum fever, and low socioeconomic status $[585,586]$. Patients with low socioeconomic status may have received inadequate prenatal care.

The factor most frequently associated with infectious morbidity in postcesarean delivery is prolonged labor in the presence of ruptured membranes. Intact chorioamniotic membranes serve as a protective barrier against bacterial infection. Rupture of the membrane exposes the uterine surface to bacteria from the birth canal. The vaginal fluid with bacterial flora is drawn into the uterus when it relaxes between contractions during labor. Women undergoing labor for more than six to eight hours in the presence of ruptured membranes should be considered at high risk for developing endometritis [587]. Other risk factors for SSIs after cesarean delivery include systemic illness, poor hygiene, obesity, and anemia $[587,588]$.

\section{Organisms}

The normal flora of the vagina include staphylococci, streptococci, enterococci, lactobacilli, diphtheroids, E. coli, anaerobic streptococci (Peptococcus species and Peptostreptococcus species), Bacteroides species (e.g., Bacteroides bivius, B. fragilis), and Fusobacterium species [584,587,589-592]. Endometritis infections are often polymicrobial and include aerobic streptococcus (particularly group B $\beta$-hemolytic streptococcus and enterococci), gram-negative aerobes (particularly E. coli), gram-negative anaerobic rods (particularly B. bivius), and anaerobic cocci (Peptococcus species and Peptostreptococcus species). Ureaplasma urealyticum has been commonly isolated from endometrial and surgical-site cultures. Additional commonly isolated organisms from SSIs include Staphylococcus species and enterococci.

\section{Efficacy}

While the use of antimicrobial prophylaxis in low-risk procedures (i.e., those with no active labor and no rupture of membranes) has been brought into question by the results of several randomized, placebo-controlled studies that found no reduction in infectious complications (fever, SSI, urinary tract infection, or endometritis) with the use of prophylaxis, the majority of these evaluations were underpowered and included administration of antimicrobial prophylaxis at cord clamping [593-599]. However, the efficacy of antimicrobial prophylaxis in cesarean delivery has been shown in several studies and two meta-analyses for both elective and nonelective procedures. Therefore, prophylaxis is recommended for all patients undergoing cesarean delivery [584,592].

One meta-analysis that reviewed seven placebo-controlled randomized trials in low-risk elective cesarean delivery found that prophylaxis was associated with a significant decrease in endometritis and fever [592]. A larger meta-analysis of 81 randomized trials with 11,937 women undergoing both elective and nonelective cesarean delivery found that antimicrobial prophylaxis was associated with a significant reduction in risk of fever, endometritis, SSI, urinary tract infection, and serious infection [585]. The relative risk for endometritis in elective cesarean section was 0.38 (95\% CI, $0.22-0.64)$ in those receiving antimicrobial prophylaxis compared to those receiving no prophylaxis.

Choice of agent. Although several different antimicrobials used alone or in combination for antimicrobial prophylaxis during cesarean delivery have been evaluated, the use of firstgeneration cephalosporins (specifically cefazolin) has been advocated by the American College of Obstetrics and Gynecology (ACOG) and the American Academy of Pediatrics (AAP), based on their efficacy, narrow spectrum of activity, and low cost [584]. This recommendation is supported by a meta-analysis of 51 randomized controlled trials comparing at least two antimicrobial regimens that concluded that ampicillin and first-generation cephalosporins have similar efficacy [600].

Newer prospective randomized controlled and cohort studies have evaluated the addition of metronidazole, azithromycin [601-603], or doxycycline [601] to a first- or second-generation cephalosporin to extend the spectrum of activity against common organisms isolated from endometrial and surgical-site cultures, specifically $U$. urealyticum and Mycoplasma species. These studies found significantly lower rates of postoperative infections (including endometritis and SSI) and a shorter duration of hospital stay compared with prophylaxis with a first- or secondgeneration cephalosporin alone [601-604]. Antibiotic administration occurred either postoperatively or after cord clamping in these studies. Further study, particularly with preoperative antimicrobial administration, is needed to confirm these preliminary findings and establish a place in therapy for this practice. 
Timing. Historically, administration of antimicrobials in cesarean delivery was delayed until after cord clamping $[600,605,606]$. The principal reasons were to avoid suppression of the neonate's normal bacterial flora that could promote the selection of resistant organisms and concern that the antimicrobials could potentially mask neonatal infection, complicating evaluation of neonatal sepsis. However, more contemporary data support the administration of antimicrobial prophylaxis before surgical incision to protect against bacterial contamination of the surgical site and decrease the risk of infection. The practice of antimicrobial prophylaxis administration before surgical incision is endorsed by ACOG and AAP [584,607]. See the Common Principles section of these guidelines for additional discussion on antimicrobial timing.

A meta-analysis of three randomized controlled trials and two nonrandomized controlled studies provided evidence that preoperative antimicrobial administration significantly decreased the rate of endometritis compared with administration after cord clamping $(3.9 \%$ and $8.9 \%$, respectively; $\mathrm{p}=0.012$ ) [605]. A lower SSI rate was also seen with preoperative antimicrobial administration $(3.2 \%$ versus $5.4 \%)$, though this difference was not significant. The overall rate of infection-related morbidity was also significantly lower. No differences between the groups were seen in neonatal outcomes, including sepsis, sepsis workups, and neonatal intensive care unit admissions. The largest study included in this meta-analysis was a prospective, randomized, controlled, double-blind, single-center, double-dummy study of 357 patients comparing cefazolin $1 \mathrm{~g}$ i.v. given preoperatively and after cord clamping, which had results consistent with the overall meta-analysis [606].

In a recent randomized trial of more than 1,100 women undergoing cesarean section between 2004 and 2010, Witt and colleagues [608] found no difference in SSI rates for patients having antimicrobial administration before surgical incision compared with those who received antimicrobial prophylaxis at the time of cord clamping. All patients received a single dose of cefazolin $2 \mathrm{~g}$.

Duration. A meta-analysis of 51 studies found that multidose regimens provided no apparent benefit over singledose regimens [600]. The use of single-dose prophylaxis is supported by ACOG and AAP for procedures lasting less than two hours [584]. Additional intraoperative doses may be warranted for patients with excessive blood loss or for whom the duration of the procedure is extended. For additional discussion of dosing, see the Common Principles section of these guidelines.

\section{Recommendation}

The recommended regimen for all women undergoing cesarean delivery is a single dose of cefazolin administered before surgical incision (Table 2). (Strength of evidence for prophylaxis $=$ A.) For patients with $\beta$-lactam allergies, an alternative regimen is clindamycin plus gentamicin.

\section{Hysterectomy procedures}

\section{Background}

Hysterectomy is second only to cesarean delivery as the most frequently performed major gynecologic procedure in the United States, with over 600,000 hysterectomies performed annually [609]. Uterine fibroid tumors account for $40 \%$ of all presurgical diagnoses leading to hysterectomy [609]. Other common diagnoses are dysfunctional uterine bleeding, genital prolapse, endometriosis, chronic pelvic pain, pelvic inflammatory disease, endometrial hyperplasia, and cancer.

Hysterectomy involves the removal of the uterus and, occasionally, one or two fallopian tubes, the ovaries, or a combination of ovaries and fallopian tubes [610]. Radical hysterectomy entails removal of the uterus, fallopian tubes, and ovaries and extensive stripping of the pelvic lymph nodes in patients with extension of their cancer. Hysterectomies are performed by a vaginal or abdominal approach using a laparoscopic- or robot-assisted method. During a vaginal hysterectomy, the procedure is completed through the vagina with no abdominal incision. Abdominal hysterectomy involves an abdominal incision. Laparoscopic and robotic methods involve small incisions and require additional equipment, increased operator experience, and increased length of procedures $[611,612]$. In the United States, between 2000 and 2004, the abdominal approach for hysterectomy was used in $67.9 \%$ of surgical procedures and the vaginal approach in $32.1 \%$. Of hysterectomies performed via the vaginal approach, 32.4\% also used laparoscopy [609]. The ACOG Committee on Gynecologic Practice recommends vaginal hysterectomy as the approach of choice for benign disease, based on evidence of better outcomes and fewer complications [613]. Laparoscopic abdominal hysterectomy is an alternative when the vaginal route is not indicated or feasible [613,614]. Of note, ACOG has stated that the supracervical approachremoval of the uterus with preservation of the cervix-should not be recommended as a superior technique for hysterectomy due to the lack of advantage in postoperative complications, urinary symptoms, or sexual function and the increased risk of future trachelectomy to remove the cervical stump [615].

Infections after hysterectomy include superficial and organ/space (vaginal cuff infection, pelvic cellulitis, and pelvic abscess) SSIs [589]. The reported SSI rates between January 2006 and December 2008 in the United States, based on NNIS risk index category, were $0.73-1.16$ per 100 procedures for vaginal hysterectomy and 1.10-4.05 per 100 procedures for abdominal hysterectomy [165]. A multicenter surveillance study found a mean infection rate of $2.53 \%$ associated with all types of hysterectomy and a significantly lower mean rate of infection with laparoscopic versus abdominal hysterectomies (1.15\% versus $3.44 \%$, respectively) [325].

Risk factors for infection after vaginal or abdominal hysterectomy include longer duration of surgery, young age, diabetes, obesity, peripheral vascular disease, collagen disease, anemia, transfusion, poor nutritional status, and previous history of postsurgical infection [590,616-622]. The depth of subcutaneous tissue is also a significant risk factor for infection after abdominal hysterectomy [623]. Additional risk factors for infection after radical hysterectomy for cervical cancer include the presence of malignancy, prior radiation therapy, and the presence of indwelling drainage catheters $[619,620]$.

\section{Organisms}

The vagina is normally colonized with a wide variety of bacteria, including gram-positive and gram-negative aerobes and anaerobes. The normal flora of the vagina includes 
staphylococci, streptococci, enterococci, lactobacilli, diphtheroids, E. coli, anaerobic streptococci, Bacteroides species, and Fusobacterium species $[589,624]$. Postoperative vaginal flora differs from preoperative flora; the amount of enterococci, gram-negative bacilli, and Bacteroides species increases postoperatively. Postoperative changes in flora may occur independently of prophylactic antimicrobial administration and are not by themselves predictive of postoperative infection $[589,625,626]$. Postoperative infections associated with vaginal hysterectomy are frequently polymicrobial, with enterococci, aerobic gram-negative bacilli, and Bacteroides species isolated most frequently. Postoperative SSIs after abdominal and radical hysterectomies are also polymicrobial; gram-positive cocci and enteric gram-negative bacilli predominate, and anaerobes are frequently isolated [626,627].

\section{Efficacy}

A meta-analysis of 25 randomized controlled trials demonstrated the efficacy of antimicrobial prophylaxis, including first- and second-generation cephalosporins and metronidazole, in the prevention of infections after abdominal hysterectomy [628]. The infection rates were $21.1 \%$ with placebo or no prophylaxis and $9.0 \%$ with any antimicrobial. Another meta-analysis found that the rate of postoperative infection (surgical and pelvic sites) in women undergoing vaginal hysterectomy who received placebo or no prophylactic antimicrobial ranged from $14 \%$ to $57 \%$, which was significantly higher than the $10 \%$ rate reported with antimicrobials [629].

Malignant disease as the reason for hysterectomy is a common exclusion from studies of antimicrobial prophylaxis. Older, prospective, placebo-controlled studies found a lower rate of SSIs with antimicrobial prophylaxis after radical hysterectomy [619,630-633]. The applicability of these results is limited by small sample size and the inclusion of antimicrobials not available in the United States. Radical hysterectomy is primarily completed through an abdominal approach but can also be performed by a vaginal approach and using laparoscopic or robotic methods [634]. Therefore, antimicrobial prophylaxis would be warranted, regardless of approach. No placebo-controlled studies have been conducted to evaluate the efficacy of antimicrobial prophylaxis when used for laparoscopic hysterectomy.

Choice of agent. Cephalosporins are the most frequently used and studied antimicrobials for prophylaxis in vaginal and abdominal hysterectomies. Studies directly comparing different cephalosporins have found no significant differences in rates of infection in vaginal hysterectomy and have indicated that first-generation cephalosporins (primarily cefazolin) are equivalent to second- and third-generation agents [635644]. In abdominal hysterectomy, no significant differences in the rates of serious infections were noted between second-and third-generation cephalosporin regimens [641,645-649]. Few comparisons have been made between second-generation cephalosporins and cefazolin. Cefazolin has been at least as effective in preventing infectious complications as secondand third-generation cephalosporins [636,650-652]. However, one double-blind controlled study of 511 women undergoing abdominal hysterectomy found that the risk of major SSIs requiring antimicrobial therapy was significantly higher in the group receiving preoperative cefazolin $1 \mathrm{~g}(11.6 \%$; relative risk, $1.84 ; 95 \%$ CI, 1.03-3.29) than in those treated with cefo- tetan $1 \mathrm{~g}(6.3 \%)$ [617]. A multicenter, randomized, doubleblind, active- and placebo-controlled study compared single doses of ampicillin, cefazolin, and placebo administered to women undergoing elective total abdominal hysterectomy at two centers in Thailand [653]. The study found a significantly lower rate of infection, including superficial and deep SSIs, urinary tract infections, vaginal cuff infection, and pneumonia, with cefazolin $(10.3 \%)$ compared with placebo $(26.9 \%)$ and ampicillin $(22.6 \%)$. No difference was seen between ampicillin and placebo. The study authors concluded that cefazolin was more effective than ampicillin for elective total abdominal hysterectomy.

A randomized controlled study of 511 patients undergoing laparoscopic gynecological procedures at one center in Italy compared single doses of amoxicillin-clavulanate $2.2 \mathrm{~g}$ and cefazolin $2 \mathrm{~g}$ i.v. administered 20-30 min before the procedure [654]. A second dose was given if the surgery lasted over three hours or there was extensive blood loss $(>1,500 \mathrm{~mL})$. No significant differences in the rates of any postoperative infection, including SSIs, were found between groups. The statistical power of the study was not stated.

In light of the organisms encountered in the vaginal canal and comparative studies conducted among different classes of cephalosporins, cefazolin, cefotetan, cefoxitin, cefuroxime, and ampicillin-sulbactam have been supported as appropriate first-line choices for prophylaxis during vaginal or abdominal hysterectomy $[6,9,41]$. Alternative agents for patients with a history of immediate hypersensitivity to penicillin include either clindamycin or metronidazole plus an aminoglycoside or a fluoroquinolone (ciprofloxacin, levofloxacin, or moxifloxacin) or aztreonam (with clindamycin only).

Duration. Studies comparing single doses of one antimicrobial with multidose regimens of a different antimicrobial have shown the two regimens to be equally effective in reducing the postoperative infection rate in women undergoing vaginal and abdominal hysterectomies [635-643,645-650, 655-663]. The limited comparative trials involving singledose cefazolin $[637,654,655,664]$ or ampicillin-sulbactam $[654,663]$ indicate that a single dose of antimicrobial is sufficient prophylaxis for SSIs for vaginal hysterectomy. Single doses of cefotetan, ceftizoxime, or cefotaxime appear to be as effective as multiple doses of cefoxitin [644-649,665]. A second dose of antimicrobial is warranted when the procedure lasts three hours or longer or if blood loss exceeds $1,500 \mathrm{~mL}[9,654]$.

\section{Recommendation}

The recommended regimen for women undergoing vaginal or abdominal hysterectomy, using an open or laparoscopic approach, is a single dose of cefazolin (Table 2). Cefoxitin, cefotetan, or ampicillin-sulbactam may also be used. Alternative agents for patients with a $\beta$-lactam allergy include (1) either clindamycin or vancomycin plus an aminoglycoside, aztreonam, or a fluoroquinolone and (2) metronidazole plus an aminoglycoside or a fluoroquinolone. (Strength of evidence for prophylaxis $=$ A.)

\section{Ophthalmic procedures}

\section{Background}

Ophthalmic procedures include cataract extractions, vitrectomies, keratoplasties, intraocular lens implantation, 
glaucoma procedures, strabotomies, retinal detachment repair, laser in situ keratomileusis, and laser-assisted subepithelial keratectomy. Most of the available data regarding antimicrobial prophylaxis involve cataract procedures. The goal of prophylaxis is primarily to reduce acute postoperative endophthalmitis, defined as severe intraocular inflammation due to infection, which can lead to loss of vision if untreated [666]. Since 2000, the reported frequency of endophthalmitis after ophthalmic procedures is low worldwide, ranging from zero to $0.63 \%$ [667-680]. The reported time from procedure to diagnosis of endophthalmitis ranges from one day to six weeks, with the majority of infections identified within one week [666,669,671,673,674,681-683].

Potential risk factors for postoperative ophthalmic infections include preoperative factors such as diabetes [666], active ocular infection or colonization [666,684], lacrimal drainage system infection or obstruction, age of $>85$ years [685], and immunodeficiency [684]. Procedure-related risk factors include clear corneal incisions (as opposed to scleral tunnel incisions) [680,686], any surgical complication, vitreous loss [684], posterior capsule tear [681,684,685], silicone intraocular lens implantation $[677,680]$, and the nonuse of facemasks in the operating theater [681].

\section{Organisms}

Among organisms isolated from patients developing postoperative endophthalmitis after cataract procedure, approximately 25-60\% were coagulase-negative Staphylococcus species, primarily S. epidermidis $[668,670,671,673,674,678,683$, $684,686]$. Other gram-positive organisms identified included S. aureus, Streptococcus species, Enterococcus species, P. acnes, and Corynebacterium species. Gram-negative organisms isolated included Serratia species, Klebsiella species, P. mirabilis, and $P$. aeruginosa. These organisms represent the normal flora isolated preoperatively in a number of studies [675,687-693].

\section{Efficacy}

Data on antimicrobial prophylaxis efficacy in ophthalmic procedures to prevent endophthalmitis are limited; however, prophylaxis is common [684]. The low rate of postoperative endophthalmitis makes it difficult to complete an adequately powered study to show efficacy of antimicrobial prophylaxis in ophthalmic procedures; therefore, surrogate markers of eradication of normal flora bacteria and reduction of bacterial count on the conjunctiva, lower and upper eyelids, eyelashes, and inner canthus (corner of the eye) preoperatively and postoperatively are used. Many of the available studies are flawed with retrospective or uncontrolled design, inadequate follow-up, variations in surgical techniques (including disinfection, antimicrobial prophylaxis strategies, and methods for performing procedures), and limited reporting of clinical outcomes.

The large, randomized, partially-masked, placebocontrolled, multinational, multicenter study conducted by the European Society of Cataract and Refractive Surgeons (ESCRS) compared the rate of postoperative endophthalmitis in over 16,600 patients undergoing routine cataract procedures at 24 centers in Europe randomized to one of four perioperative prophylaxis groups $[679,680,694]$. Patients received no antimicrobial prophylaxis, intracameral cefuroxime at the end of the procedure alone, perioperative levofloxacin $0.5 \%$ ophthalmic solution given within the hour before the procedure, or both intracameral cefuroxime and perioperative levofloxacin. All patients had the eye area disinfected with povidone-iodine $5 \%$ preoperatively and received topical levofloxacin postoperatively. The study was stopped after an interim analysis due to results of a multivariable analysis indicating that patients not receiving intracameral cefuroxime were approximately five times more likely to develop endophthalmitis. The study has been questioned for its high rate of endophthalmitis, selection of cefuroxime due to gaps in gram-negative coverage, unknown drug concentrations in the aqueous humor, risks of hypersensitivity, the lack of a commercially available preparation, the lack of a subconjunctival cefuroxime treatment group, selection of topical levofloxacin, and methods for statistical analysis [695-697].

Two single-center, historical-controlled studies in hospitals in Spain reported decreases in acute postoperative endophthalmitis among patients undergoing a cataract procedure with intracameral cefazolin added to the previous routine prophylaxis of preoperative eyelid cleansing with soap for three days [670] and povidone-iodine eye area preparation [670,674], topical antimicrobial, and corticosteroid preparations given at the end of the procedure and postoperatively. One study found a significant decrease and a relative risk reduction of $88.7 \%$ in postoperative endophthalmitis with intracameral cefazolin [670]. The other found a decrease from $0.63 \%$ to $0.055 \%$ in postoperative endophthalmitis with intracameral cefazolin [674]. No statistical analysis was performed in this study.

A retrospective cohort study of patients undergoing cataract procedure at one center in Canada between 1994 and 1998 found no significant difference in the rate of postoperative endophthalmitis with preoperative topical antimicrobials compared with none [668]. A significant decrease in endophthalmitis was seen with subconjunctival administration of antimicrobials at the end of the procedure compared with no antimicrobials.

Several prospective studies have shown decreases in ocular flora, measured by bacterial isolate and CFU counts, with preoperative antimicrobial irrigation [675], topical antimicrobials [687,688,691,692,698-700], and intracameral antimicrobials [682]. These studies did not report rates of endophthalmitis, limiting the application of the results.

Choice of agent. Along with careful site preparation and disinfection, the ideal antimicrobial prophylaxis agent should be bactericidal against common pathogens of postoperative endophthalmitis and be used safely in the eye $[6,8,684]$. There is no consensus on the agent of choice for antimicrobial prophylaxis in ophthalmic procedures, and no agent is FDAapproved for this indication. There are limited studies evaluating the efficacy of a particular choice of antimicrobial prophylaxis for ophthalmic surgeries. The most efficacious antimicrobial cannot be determined from the available data due to study flaws and a lack of direct comparisons. Local ocular flora resistance patterns should be monitored to aid in the selection of appropriate agents for prophylaxis [683,689,701].

Based on the available literature, use of povidone-iodine as a preoperative antiseptic agent is recommended to decrease ocular microbes and thereby prevent endophthalmitis $[6,684$, 702]. Povidone-iodine $5 \%$ or $10 \%$ is instilled in the conjunctival sac and applied topically to the ocular skin surface [703]. The most effective protocol has not been established, as 
povidone-iodine is frequently used in combination with other antimicrobials [670,674,675,678,687,704]. Chlorhexidine has been used as an effective alternative to povidone-iodine, particularly in patients who are iodine-allergic [682,703].

Ophthalmic surgeons surveyed in the United Kingdom reported that commonly used antimicrobial prophylactic agents included cephalosporins, aminoglycosides, vancomycin, chloramphenicol, neomycin alone or in combination with polymyxin, and fluoroquinolones [695,703]. A similar survey of members of the American Society of Cataract and Refractive Surgery found that over $90 \%$ of respondents used fluoroquinolones (mainly fourth-generation agents), vancomycin, and cephalosporins [697]. These antimicrobials have been recommended in practice guidelines [6].

Cephalosporins, specifically cefazolin, cefuroxime, and ceftazidime, have been shown to be safe and effective in decreasing postoperative endophthalmitis when added to regimens of povidone-iodine and topical antimicrobials $[670,674,677,679,680,699]$. Vancomycin has been shown to decrease cultures and reach adequate concentrations to prevent and treat most corneal pathogens [675,705]. Aminoglycosides alone [687] or in combination with an antiseptic agent (chlorhexidine) [682] showed no significant difference in the reduction of culture results compared with an antiseptic alone (povidone-iodine or chlorhexidine) [682,690] and no antimicrobial prophylaxis.

A randomized controlled study compared the antimicrobial activity and safety of trimethoprim $0.1 \%$-polymyxin B sulfate 10,000 units/mL ophthalmic solution and tobramycin $0.3 \%$ ophthalmic solution in patients undergoing cataract procedures [692]. All patients received one drop and a subconjunctival injection of corticosteroids and gentamicin postoperatively followed by one drop of study medication four times daily for five to seven days. No significant differences were seen between groups for positive culture results from conjunctiva at baseline, at procedure, or at postoperative days 5-7 or in lid margin culture at baseline and postoperative days 5-7. A higher rate of positive cultures at procedure was seen in the trimethoprim-polymyxin group (37 of 59 cultures, $63 \%$ ) compared with 13 (41\%) of 32 cultures in the tobramycin group ( $p=0.043$ ). Both medications eradicated the majority of bacteria on the day of procedure and postoperative days 5-7. Aqueous humor concentrations did not achieve the minimum inhibitory concentrations (MICs) of S. aureus or S. epidermidis and were undetectable for polymyxin B sulfate. The adverse events of irritation and allergic reaction were experienced by three patients in the trimethoprim-polymyxin group. The study authors concluded that there was no difference between trimethoprim and tobramycin in ocular flora reduction.

A randomized controlled study compared conjunctiva and contact lens culture results after treatment with tobramycin $0.3 \%$ versus ofloxacin $0.3 \%$ ophthalmic solutions in patients undergoing photorefractive keratectomy [693]. No differences were seen among preoperative, postoperative, or contact lens cultures between treatment groups. Although not statistically significant, logistic regression found that cultures from patients treated with tobramycin were two times more likely to be positive than those treated with ofloxacin. The study had low power and did not compare baseline and posttreatment culture results for any treatment group.

Fluoroquinolones have been found in studies to significantly decrease the ocular culture results from baseline
[667,673,691,698,700,706]; achieve aqueous humor, vitreal, and corneal tissue concentrations adequate to prevent and treat common ocular pathogens [705,707-710]; and result in improved ocular measurements (i.e., visual acuity, epithelial cell counts, and epithelial healing) [711-716]. A retrospective multicenter case series of 20,013 patients who underwent uncomplicated cataract surgeries and received fourth-generation fluoroquinolones preoperatively and postoperatively reported the rates of postoperative endophthalmitis [673]. Endophthalmitis occurred in $9(0.06 \%)$ of 16,209 surgeries in patients treated with gatifloxacin $0.3 \%$ ophthalmic solution (95\% CI, $0.03-0.1 \%)$ and in $5(0.1 \%)$ of 3,804 surgeries in patients treated with moxifloxacin $0.5 \%$ ophthalmic solution (95\% CI, 0.05-0.3\%). There were no significant differences in efficacy between agents. In a retrospective cross-sectional study conducted over a 10-year period with third- and fourthgeneration fluoroquinolones, significantly lower rates of endophthalmitis were reported for the fourth-generation agents moxifloxacin and gatifloxacin (0.56 per 1000 cataract surgeries) than for the third-generation agents ciprofloxacin and ofloxacin (1.97 per 1000 surgeries) $(p=0.0011)$ [671].

Route. There is no consensus on the most effective route of antimicrobial administration for the prevention of endophthalmitis. The routes of antimicrobial administration used in ophthalmic procedures include preoperative topical antimicrobial ophthalmic drops, addition of antimicrobials to the irrigation solution, instillation of antimicrobials intracamerally at the end of surgery, subconjunctival injection of antimicrobials, and postoperative topical application of antimicrobials [6,684,702,717].

The ESCRS randomized controlled study mentioned above found that patients not receiving intracameral cefuroxime were approximately six times more likely to develop postoperative endophthalmitis $[679,680,694]$. Surveys of the impact of the ESCRS study findings found that there was an increase in the use of intracameral over subconjunctival cefuroxime based on preliminary study results [703]. For respondents who had not adopted this practice, the reported reasons for not using intracameral cefuroxime included the need for further study, concerns about risk and cost of therapy, the lack of a subconjunctival comparator group, the high rate of endophthalmitis in the control groups, concerns about statistical analysis, and questions regarding the selection of cefuroxime due to gaps in ophthalmic pathogen coverage $[695,697]$. There is no commercially available cefuroxime formulation for intracameral administration, which was reported as one of the main barriers to use of this route. Concerns regarding compounded intracameral antimicrobials expressed by survey respondents included inflammation, dilution errors, corneal endothelial injury, and the risk for bacterial contamination and infection.

A retrospective cohort study compared the efficacy of intracameral cefuroxime versus subconjunctival cefuroxime in reducing the rate of endophthalmitis after cataract procedures at one center in northeast England [718]. A total of 19,425 patients received antimicrobial prophylaxis with preoperative povidone-iodine $5 \%$ in the conjunctival sac and subconjunctival injection of cefuroxime $50 \mathrm{mg}$ at the end of the procedure, and 17,318 patients received intracameral cefuroxime $1 \mathrm{mg}$ at the end of the procedure. There were two 
groups of patients excluded from the analysis: Protocol violators who received no prophylaxis and patients who were enrolled in the ESCRS study. The overall rate of endophthalmitis in analyzed patients was 35 cases in 36,743 procedures ( 0.95 per 1,000 cases). Of these, 27 occurred in the subconjunctival cefuroxime group ( 1.39 per 1,000 cases), and 8 occurred in the intracameral group ( 0.46 per 1,000 cases) $(\mathrm{OR}$, $3.01 ; 95 \% \mathrm{CI}, 1.37-6.63 ; \mathrm{p}=0.0068)$.

Several studies found a lower rate of endophthalmitis with the addition of intracameral cephalosporins (cefazolin and cefuroxime) at the end of the surgical procedure after routine perioperative and postoperative topical antimicrobial prophylaxis regimens $[670,674]$. A case-control study revealed a 5.7-times increased likelihood of developing postoperative endophthalmitis with topical antimicrobial prophylaxis only (including gentamicin $0.3 \%$ and chlorhexidine $0.05 \%$ ) compared with the addition of intracameral cefuroxime $1 \mathrm{mg}$ to the regimen in cataract procedure [677]. Both intracameral cephalosporins and moxifloxacin have been shown as safe, with no adverse events and no effects on visual acuity and endothelial cell counts $[670,674,699,715,716]$.

One study involving healthy adult volunteers found that orally administered levofloxacin and moxifloxacin achieved adequate aqueous humor concentrations to provide activity against gram-positive and most gram-negative ocular pathogens without adverse events [707]. The addition of subconjunctival antimicrobials to existing topical antimicrobial prophylaxis regimens has also been shown to reduce the rate and risk of endophthalmitis in intraocular procedures compared with topical antimicrobials alone $[668,681,686]$. Topical antimicrobials have been shown to be safe and effective in lowering rates of endophthalmitis [671,673], decreasing bacterial organisms and CFUs in conjunctiva [667,675,691, $692,698,700]$, and achieving adequate concentrations to be effective against most ocular pathogens [705,706,708-710,719], with no notable adverse events [711-714].

Duration and timing. There are a lack of clear evidence and no consensus on the appropriate duration and timing of antimicrobial prophylaxis in ophthalmic procedures $[6,684]$. Commonly reported times of antimicrobial prophylaxis include preoperatively, intraoperatively, at the end of the procedure, and postoperatively [684]. Few studies have investigated the differences between the timing and duration of antimicrobial prophylaxis regimens. Many of the regimens are used in combination, making it difficult to determine the optimal timing and duration. Preoperative antimicrobial timing reported in the literature has ranged from one to multiple drops within an hour preoperatively on the day of the procedure $[671,673,679,680,692-694,698,703,709,710,716]$ or one to three days before the procedure $[667,698,700$, $703,708,710,712,714]$.

Two topical moxifloxacin regimens were compared for conjunctival bacterial flora and aqueous humor concentrations in a randomized controlled study of patients undergoing cataract procedures $[691,719]$. In one regimen, patients were administered moxifloxacin $0.5 \%$ four times a day beginning one day before the procedure plus one drop two hours before the procedure (total of five drops before the procedure); the other group received moxifloxacin $0.5 \%$ two hours before surgery and every 15 minutes for the first hour of the procedure (total of five drops). There were no cases of postoperative endophthalmitis up to six months after the procedure in any patient. Administration of moxifloxacin on the day of the procedure was found to result in a significant decrease in median CFU compared with baseline and was found (based on change in $\log$ CFU) to be more effective than antimicrobial administration on the day before the procedure. Mean aqueous humor concentrations of moxifloxacin at the beginning of the procedure were significantly higher in the group who received the drug on the day of the procedure.

A small, randomized controlled study compared aqueous humor concentrations of levofloxacin and ciprofloxacin in patients undergoing a cataract procedure with routine phacoemulsification given as (1) one or two drops four times daily for two days before the procedure, with the last dose given immediately before bedtime on the night before the procedure, (2) five doses (one or two drops) delivered every $10 \mathrm{~min}$ in the hour before the procedure, or (3) a combination of both dosing strategies [706]. Aqueous humor concentrations of levofloxacin were significantly higher than those of ciprofloxacin. Significantly higher doses of drug were delivered to the aqueous humor in the group receiving same-day prophylaxis than in patients receiving levofloxacin or ciprofloxacin two days before surgery. No cases of endophthalmitis or ocular or systemic toxicities were reported.

A randomized controlled study compared the effectiveness of topical ofloxacin in the reduction or elimination of conjunctival bacterial flora when given as one drop every five minutes for three applications one hour before the procedure alone (control group) or combined with ofloxacin one drop four times daily for three days (study group) before cataract procedures [688]. No differences in positive conjunctival cultures were seen between groups five days before topical antimicrobials or before the administration of ofloxacin on the day of the procedure. Significantly higher positive culture rates were seen in the control group than in the study group one hour after the administration of the preoperative antimicrobial and before povidone-iodine, immediately before the procedure, and at the conclusion of the procedure. Mean CFU counts did not significantly differ five days preoperatively and immediately before the procedure but were significantly higher in the control group at all other time points. Neither outcomes of endophthalmitis nor patient compliance with antimicrobial use was reported. The study's authors concluded that three days of topical ofloxacin was more effective than administration just one hour before the procedure in reducing the number of positive bacterial cultures at several time points perioperatively.

Numerous studies have evaluated the efficacy of intracameral and subconjunctival injections of antimicrobials given at the end of surgery $[6,674,677,679-682,697,699,703$, $716,718]$. The most commonly reported dose of intracameral cefuroxime was $1 \mathrm{mg}[677,679,680,682,699,718]$, and the most commonly reported subconjunctival dose was $50 \mathrm{mg}$ [718]. Doses of 2.5 or $1 \mathrm{mg}$ of intracameral cefazolin were studied $[670,674]$, as were 250- and $500 \mathrm{mg}$ doses of intracameral moxifloxacin [715,716]. Postoperative dosing strategies reported in the literature include four times daily for 3-7 days $[667,670,671,673-675,679,680,692,711,712,715]$ and for up to 15 days $[713,714]$ or until the bottle was empty [716].

Despite the lack of well-controlled trials, the consequences of bacterial endophthalmitis support the use of prophylactic antimicrobials. No definitive studies have clearly delineated superiority of antimicrobial route, timing, or duration. 


\section{Recommendation}

Due to the lack of robust data from trials, specific recommendations cannot be made regarding choice, route, or duration of prophylaxis. As a general principle, the antimicrobial prophylaxis regimens used in ophthalmic procedures should provide coverage against common ocular pathogens, including Staphylococcus species and gram-negative organisms, particularly Pseudomonas species.

Preoperative antisepsis with povidone-iodine is recommended, based on available evidence. Appropriate topical antimicrobials include commercially available neomycin-polymyxin B-gramicidin solution or fluoroquinolones (particularly fourth-generation agents) given as one drop every 5-15 min for five doses within the hour before the start of the procedure (Table 2). The addition of subconjunctival cefazolin $100 \mathrm{mg}$ or intracameral cefazolin $1-2.5 \mathrm{mg}$ or cefuroxime $1 \mathrm{mg}$ at the end of the procedure is optional. While some data have shown that intracameral antimicrobials may be more effective than subconjunctival antimicrobials, there are no commercially available antimicrobials approved for these routes of administration. (Strength of evidence for prophylaxis $=$ B.)

\section{Orthopedic procedures}

\section{Background}

Orthopedic procedures considered in these guidelines include clean orthopedic procedures (not involving replacement or implantations), spinal procedures with or without instrumentation, repair of hip fractures, implantation of internal fixation devices (screws, nails, plates, and pins), and total-joint-replacement procedures. Grade III open fractures (extensive soft tissue damage and crushing) are often associated with extensive surgical site contamination and are routinely managed with empirical antimicrobial treatment and surgical debridement, for which guidelines have been published separately [720]. Available guidelines recommend that antimicrobial prophylaxis in grade I (clean wound with $\leq 1 \mathrm{~cm}$ laceration) and grade II (clean wound with $>1 \mathrm{~cm}$ laceration without extensive soft tissue damage) open fractures be handled similarly to other clean orthopedic procedures [721-724].

Between 2006 and 2008, SSIs were reported nationally, based on risk category, in approximately $0.70-4.15$ per 100 procedures for patients undergoing spinal fusion, $0.72-2.30$ per 100 procedures in patients undergoing laminectomy, $0.67-2.40$ per 100 procedures in patients undergoing hip prosthesis, and $0.58-1.60$ per 100 procedures in patients undergoing knee prosthesis [165]. Postoperative SSI is one of the most costly complications of orthopedic procedures due to hospital readmissions, extended hospital length of stay, the need for additional procedures (often removal and reimplantation of implanted hardware), convalescent or nursing home care between procedures, and significant increases in direct hospital costs (e.g., prolonged antimicrobial therapy) $[725,726]$. Studies have found that the estimated economic impact of one deep SSI was $\$ 100,000$ in hospital cost alone after hip arthroplasty and \$60,000 after knee arthroplasty [727-731].

In light of the serious consequences, antimicrobial prophylaxis is well accepted in procedures involving the implantation of foreign materials [8,732]. Prophylaxis is also indicated in spinal procedures without instrumentation, where an SSI would pose catastrophic risks [726,733-738].

\section{Organisms}

Skin flora are the most frequent organisms involved in SSIs after orthopedic procedures. The most common pathogens in orthopedic procedures are $S$. aureus, gram-negative bacilli, coagulase-negative staphylococci (including S. epidermidis), and $\beta$-hemolytic streptococci [739-743]. Spinal procedures may be complicated by polymicrobial infection that includes gram-negative bacteria [740].

A contributing factor to SSIs in arthroplasty is the formation of bacterial biofilm, particularly with $S$. aureus and $S$. epidermidis, on inert surfaces of orthopedic devices. Bacterial biofilm confers antimicrobial resistance and makes antimicrobial penetration difficult [744-748].

There is increasing concern regarding the emergence of SSIs due to resistant microorganisms, specifically VRE and MRSA in surgical patients. Several studies have investigated MRSA colonization and SSIs and evaluated the effect of decolonization, including the use of topical mupirocin, in orthopedic procedures [150,157,741,749-753]. Mupirocin decolonization protocols as an adjunct to i.v. cephalosporin prophylaxis in orthopedic patients resulted in significant decreases in nasal MRSA carriage [150,751] and overall SSIs [157,750-752]. Preoperative decolonization with intranasal mupirocin may have utility in patients undergoing elective orthopedic procedures who are known to be colonized or infected with either MRSA or MSSA [150,151,157,741,749-755]. Readers are referred to additional discussion in the Common Principles section of these guidelines.

\section{Clean orthopedic procedures not involving implantation of foreign materials}

\section{Background}

In clean orthopedic procedures, such as knee, hand, and foot procedures, and those not involving the implantation of foreign materials, the need for antimicrobial prophylaxis is not well established [738,749,756]. Antimicrobial prophylaxis in patients undergoing diagnostic and operative arthroscopic procedures is controversial [6,757-760]. The risks of SSI and long-term sequelae are low for procedures not involving implantation.

\section{Efficacy}

The efficacy of antimicrobial prophylaxis in clean orthopedic procedures was first investigated in the middle part of the 20th century. A number of these studies and reviews have since been found to be flawed, as patients were not randomized to treatment groups and the timing and duration of antimicrobial prophylaxis were not studied [761,762]. Further, patients were administered prophylactic antimicrobials after the surgical procedure, which may have led to invalid results. The low rate of infection and absence of serious morbidity failed to justify the expense or potential for toxicity and resistance associated with routine use of antimicrobial prophylaxis in the setting of clean orthopedic procedures.

\section{Recommendations}

Antimicrobial prophylaxis is not recommended for patients undergoing clean orthopedic procedures, including knee, 
hand, and foot procedures, arthroscopy, and other procedures without instrumentation or implantation of foreign materials. (Strength of evidence against prophylaxis $=\mathrm{C}$.) If the potential for implantation of foreign materials is unknown, the procedure should be treated as with implantation.

\section{Spinal procedures with and without instrumentation}

\section{Background}

Data support the use of antimicrobial prophylaxis for orthopedic spinal procedures with and without instrumentation, including fusions, laminectomies, and minimally invasive disk procedures, to decrease the rate of postoperative spinal infection $[8,543,563,732,733,739,763-766]$. Surgical site infections after orthopedic spinal procedures, including minimally invasive disk procedures, are associated with high morbidity. Invasion of the epidural space in organ/space SSIs is of particular concern after spinal procedures [8,145,767].

Surgical site infection rates vary with the complexity of the procedure. One retrospective, multicenter study of 1274 adult patients found an overall SSI rate of $0.22 \%$ with antimicrobial prophylaxis after minimally invasive spinal procedures (i.e., any spinal procedures performed through a tubular retractortype system) [768]. Procedures included simple decompressive procedures (such as microscopic or endoscopic discectomy or foraminotomy or decompression of stenosis), minimally invasive arthrodeses with percutaneous instrumentation, and minimally invasive intradural procedures. The SSI rate in patients receiving antimicrobial prophylaxis undergoing spinal procedures with instrumentation has ranged from $2.8 \%$ to $9.7 \%$ [165,764,765,769,770]. Monosegmental instrumentation has a reported SSI rate of $<2 \%$, compared with $6.7 \%$ for instrumentation at multiple levels [771].

Several case-control studies of adults undergoing spinal procedures with and without instrumentation have found the following notable patient-related risk factors for SSI: prolonged preoperative hospitalization [771], diabetes [767,772775], elevated serum glucose concentration $(>125 \mathrm{mg} / \mathrm{dL}$ preoperatively [within 30 days] or $>200 \mathrm{mg} / \mathrm{dL}$ postoperatively) [773], older age $[767,776]$, smoking and alcohol abuse [776], previous procedure complicated by infection [774-776], and obesity [770-775,777]. Procedure-related risk factors include extended duration of procedure (defined in studies as two to five hours or greater than five hours [775], greater than three hours [771], and greater than five hours [776]), excessive blood loss (>1 L) [771,775], staged procedure [776], multilevel fusions [777], foreign-body placement (e.g., screw, rod, plate) [767], combined anterior and posterior fusion [776], and suboptimal antimicrobial timing (>60 min before or after incision) [773]. A significant decrease in SSIs was seen with procedures at the cervical spine level $[772,773]$ or with an anterior surgical approach [775].

\section{Efficacy}

Despite the lack of comparative studies evaluating prophylaxis for spinal procedures with and without instrumentation (implantation of internal fixation devices), antimicrobial prophylaxis is recommended due to the associated morbidity and assumed costs of SSIs [771]. A meta-analysis of six studies with 843 patients undergoing spinal procedures (types of procedures were not differentiated in the analysis) demonstrated an overall effectiveness of antimicrobial prophylaxis [732]. Antimicrobials studied included singledose or multidose regimens of $<24 \mathrm{~h}$ duration of cephaloridine (a first-generation cephalosporin no longer available in the United States), vancomycin and gentamicin, cefazolin with and without gentamicin, piperacillin, and oxacillin. The pooled SSI rate with antimicrobial prophylaxis was $2.2 \%$, compared with $5.9 \%$ in controls (OR, 0.37 ; 95\% CI, 0.17-0.78; $\mathrm{p}<0.01$ ). One randomized controlled study of 1237 adult patients undergoing spinal procedures to repair a herniated disk (hemilaminectomy, laminectomy, flavectomy, spondylosyndesis) found no significant difference in the rate of SSIs between single-dose cefuroxime $1.5 \mathrm{~g}$ i.v. (1.3\%) and placebo $(2.9 \%)$ given within 60 min before surgical incision. No significant difference was seen between treatment groups for incisional SSIs $(0.98 \%$ and $1.12 \%$, respectively) or deep SSIs $(0.33 \%$ and $0.32 \%$, respectively), but the difference in organ/ space infections was significant between groups $(0 \%$ and $1.44 \%$, respectively; $\mathrm{p}<0.01$ ) [778].

Choice of agent. There is no clearly superior antimicrobial agent or regimen for spinal procedures $[563,769]$. The antimicrobials most often studied for prophylaxis in orthopedic procedures are first-generation cephalosporins, particularly cefazolin. Cefazolin has been noted as a suitable agent for spinal procedures with its spectrum of activity (e.g., against Staphylococcus species and gram-negative bacilli such as E. coli) and adequate tissue [121] and disk concentrations [779,780].

Second- and third-generation cephalosporins offer no major advantages over first-generation agents. Their routine use is not recommended due to their higher cost and potential to promote resistance, particularly among health-careassociated gram-negative bacilli [8]. Broader coverage may be considered for instrumented fusion due to the risk of polymicrobial infections, including those caused by gram-negative bacteria $[563,769]$.

Clindamycin and vancomycin have adequate activity against the most common pathogens involved in orthopedic procedures and would be acceptable alternatives under certain circumstances, such as prophylaxis for patients with a $\beta$-lactam allergy. Vancomycin should be included with cefazolin or used as an alternative agent for routine antimicrobial prophylaxis for patients who are known to be colonized with MRSA [6,8,41,733,781].

Duration. The majority of available studies of antimicrobial prophylaxis in spinal procedures have used single doses or regimens of $<24 \mathrm{~h}$ duration [732]. There is no high-quality evidence supporting a duration of $>24 \mathrm{~h}$ [782], and some sources recommend only a single preoperative dose [8, $769,778]$.

\section{Pediatric efficacy}

While no studies have evaluated the efficacy of antimicrobial prophylaxis in pediatric patients undergoing spinal procedures with or without instrumentation, the incidence and risk factors for SSIs in this population have been reported. The frequencies of SSIs in pediatric patients undergoing spinal fusion were $3.5 \%$ ( $<18$ years old) [783], 3.8\% ( <19 years old) [784], 4.4\% (ages 1-22 years old), and 5.2\% (<17 years old) [764] for varying conditions, including Scheuermann kyphosis [784], 
myelodysplasia [764], idiopathic scoliosis [783,785], neuromuscular scoliosis [785], kyphosis [783], and spondylolisthesis [783]. The majority of patients in studies reporting antimicrobial prophylaxis received cefazolin, vancomycin, or clindamycin $[764,783,785]$.

Risk factors for SSIs after spinal procedures with instrumentation in a pediatric population include myelodysplasia [764], procedure at the sacral spine, obesity [785], ASA classification of $>2$, a complex medical condition (including spinal bifida, cerebral palsy, Marfan syndrome, achondroplasia, osteogenesis imperfecta, other unspecified genetic disease, muscular dystrophy, spinal muscular atrophy, or other debilitating myopathies) [783], and previous spinal procedures. One study found a decreased risk of infection with hypothermia (core body temperature of $<35.5^{\circ} \mathrm{C}$ for the duration of the procedure) [785].

Two studies found suboptimal antimicrobial prophylaxis as a risk factor for SSIs in spinal procedures [764,783]. Optimal antimicrobial prophylaxis was defined as cefazolin $20 \mathrm{mg} / \mathrm{kg}$ (up to $2 \mathrm{~g}$ ) given within $30 \mathrm{~min}$ [764] or $60 \mathrm{~min}$ [783] before surgical incision, vancomycin $10 \mathrm{mg} / \mathrm{kg}$ (up to $1 \mathrm{~g}$ ) given within $60 \mathrm{~min}$ [783] or $150 \mathrm{~min}$ [764] before surgical incision, or clindamycin $10 \mathrm{mg} / \mathrm{kg}$ (up to $600 \mathrm{mg}$ ) given within $60 \mathrm{~min}$ before surgical incision [783]. Intraoperative redosing was defined as appropriate for cefazolin if administered for procedures lasting more than four hours and for vancomycin or clindamycin for procedures lasting more than six hours in one study [783] and for cefazolin administered every eight hours in the other study [764]. A third study found that use of clindamycin as the perioperative antimicrobial increased the risk of SSI [785].

\section{Recommendations}

Antimicrobial prophylaxis is recommended for orthopedic spinal procedures with and without instrumentation. The recommended regimen is cefazolin (Table 2). (Strength of evidence for prophylaxis in orthopedic spinal procedures $=$ A.) Clindamycin and vancomycin should be reserved as alternative agents as described in the Common Principles section. If there are surveillance data showing that gram-negative organisms are a cause of SSIs for the procedure, practitioners may consider combining clindamycin or vancomycin with another agent (cefazolin if the patient is not $\beta$-lactam allergic; aztreonam, gentamicin, or single-dose fluoroquinolone if the patient is $\beta$-lactam allergic). Mupirocin should be given intranasally to all patients known to be colonized with S. aureus.

\section{Hip fracture repair}

\section{Background}

Data support the use of antimicrobial prophylaxis for hip fracture repair to reduce the rate of SSIs, particularly in procedures that involve internal fixation (e.g., nails, screws, plates, wires). Surgical site infections after hip fracture repair can result in extensive morbidity, including prolonged and repeated hospitalization, sepsis, persistent pain, device replacement, and possible death $[726,739,786-790]$.

\section{Efficacy}

The efficacy of antimicrobial prophylaxis in hip fracture repair has been illustrated in two meta-analyses $[787,788]$. One meta-analysis of 15 hip fracture procedure trials (the majority of procedures involved closed, proximal femoral, or trochanteric fractures with internal fixation) demonstrated that any dose and duration of prophylaxis are superior to no prophylaxis with respect to preventing SSIs (deep and superficial SSIs were analyzed together) [787]. The rate of SSIs was $10.4 \%$ in controls versus $5.39 \%$ in treatment groups. A second meta-analysis of 22 studies reiterated the efficacy of antimicrobial prophylaxis in fracture procedures [788]. The analysis included the same hip fracture studies examined in the first meta-analysis, with additional studies of long-bone fracture repair (i.e., closed ankle fracture and other closed fractures, some noted with internal fixation). This second meta-analysis reviewed 10 studies of 1,896 patients receiving a preoperative and two or more postoperative doses of a parenteral antimicrobial compared with a placebo or with no treatment. The authors found a relative risk of deep SSIs of $0.36(95 \% \mathrm{CI}, 0.21-0.65)$ and a relative risk of superficial SSIs of 0.48 (95\% CI, 0.28-0.81) associated with antimicrobial use.

Choice of agent. The antimicrobials most often studied for prophylaxis in orthopedic procedures are first-generation cephalosporins due to their ease of administration, low cost, and safety profile $[787,788,791]$. Second- and third-generation cephalosporins have not been shown to offer clear advantages over first-generation agents. These agents are not recommended for routine use due to their higher cost, potential to promote resistance, and association with adverse events (e.g., C. difficile-associated diarrhea) [8,790,792].

Alternative regimens may be needed for institutions with highly resistant organisms, such as MRSA or C. difficile. Success in decreasing rates of $C$. difficile-associated disease and mortality was seen in a single-center study with the antimicrobial prophylaxis regimen change from three doses of cefuroxime $[790,792]$ to a single preoperative dose of cefuroxime plus gentamicin [792]. In another study, C. difficile-associated disease decreased after the prophylaxis regimen was changed from cefuroxime to amoxicillin-clavulanate [790].

Clindamycin and vancomycin have adequate activity against the most common pathogens involved in orthopedic procedures and would be acceptable alternatives under certain circumstances, such as prophylaxis for patients with a $\beta$-lactam allergy. Vancomycin should be included with cefazolin or used as an alternative agent for routine antimicrobial prophylaxis for patients who are known to be colonized with MRSA [6,8,41,733,781].

Duration. For effective prophylaxis, the MIC of the antimicrobial needs to be exceeded at the target site from the moment of incision until surgical-site closure [788]. Two metaanalyses demonstrating the efficacy of antimicrobial prophylaxis in long-bone and hip fracture procedures also showed that multiple perioperative doses did not offer an advantage over a single preoperative dose $[787,788]$. These studies support a duration of antimicrobial prophylaxis of $\leq 24 \mathrm{~h}$.

\section{Recommendations}

The recommended regimen in hip fracture repair or other orthopedic procedures involving internal fixation is cefazolin. Clindamycin and vancomycin should be reserved as alternative agents, as described in the Common Principles section. 
If there are surveillance data showing that gram-negative organisms are a cause of SSIs for the procedure, practitioners may consider combining clindamycin or vancomycin with another agent (cefazolin if the patient is not $\beta$-lactam-allergic; aztreonam, gentamicin, or single-dose fluoroquinolone if the patient is $\beta$-lactam-allergic). Mupirocin should be given intranasally to all patients with documented colonization with S. aureus. (Strength of evidence for prophylaxis $=$ A.)

\section{Total joint replacement}

\section{Background}

In 2005, more than 750,000 hip or knee replacements were performed in the United States [793]. The reported frequency of SSIs complicating hip, knee, elbow, ankle, or shoulder replacement ranges from $0.6 \%$ to $12 \%$ [743,786,794-797]. Rates of SSI as high as $11 \%$ after hip replacement and $12 \%$ after elbow replacement have been reported [786,797]. However, for hip and knee replacements, the most common joint arthroplasties, infection rates are typically less than $2 \%$ [165].

The introduction of antimicrobial prophylaxis, stringent infection-control protocols, and the use of ultraclean operating rooms has led to a substantial reduction in SSI rates (to $\leq 1 \%$ ) $[734,786,796,798,799]$. Postoperative prosthetic joint infection is an organ/space SSI that occurs early (within 3 mos postoperatively), is delayed (3-12 mos postoperatively), or occurs late (>12 mos after surgery) [748]. These infections frequently require removal of the prosthesis, a prolonged course of antimicrobials, and one- or two-stage reimplantation of the prosthesis and may result in permanent disability $[796,800]$. Studies have shown an estimated economic impact of one deep SSI of $\$ 100,000$ in hospital cost alone after hip arthroplasty and $\$ 60,000$ after knee arthroplasty [727-731].

Common risk factors for prosthetic joint infection [748] include advanced age; obesity; diabetes mellitus; corticosteroid use; malignancy; rheumatoid arthritis; previous arthroplasty on the same joint; arthroplasty undertaken to treat a fracture; type of joint replaced (e.g., risk is greater for the knee than the hip); perioperative surgical-site complications, including superficial SSI; hematoma; and persistent surgicalsite drainage. Operative risk factors include ASA classification of $\geq 3$, duration of procedure exceeding the 75 th percentile for the procedure or exceeding three hours, surgical site classified as contaminated or dirty, and no systemic antimicrobial prophylaxis. Excluding the presence of a systemic antimicrobial, patients with these operative risk factors are at the greatest risk of developing an SSI. A contributing factor to SSIs in arthroplasty is the formation of bacterial biofilm, particularly with S. aureus and S. epidermidis, on inert surfaces of orthopedic devices to confer antimicrobial resistance and difficulty in antimicrobial penetration [744-748].

\section{Efficacy}

The majority of studies that have evaluated antimicrobial prophylaxis in joint replacements have been conducted in patients undergoing total hip or total knee arthroplasty [801]. There is a lack of efficacy data involving elbow, shoulder, and ankle arthroplasty; however, the same antimicrobial prophylaxis principles can be applied. In light of the serious potential consequences, antimicrobial prophylaxis is well accepted in procedures involving the implantation of foreign materials [8,543,732,733].

A meta-analysis supports the use of antimicrobial prophylaxis for SSI reduction in patients undergoing total joint replacement [801]. Of the 26 randomized controlled studies examined, 24 included patients undergoing total hip or total knee arthroplasty. The meta-analysis noted that the studies did not clearly state if the arthroplasties were primary or revision. The SSIs were defined as visible purulent exudates at the surgical site (deep or superficial) in the included studies. Seven studies ( $n=3,065$ patients) pooled to compare antimicrobial prophylaxis with placebo found a relative risk reduction of SSIs of $81 \%$.

Choice of agent. There are no data supporting superiority of one class of antimicrobials over another for antimicrobial prophylaxis in total joint replacement. A meta-analysis of studies, mainly in total hip or total knee replacement, found no difference in SSIs between cephalosporins with teicoplanin (not available in the United States) in five studies with 2,625 patients, cephalosporins and penicillin derivatives in three studies of 386 patients, and first- and second-generation cephalosporins in eight studies of 2,879 patients [801]. Selection should be based on cost, availability, and local resistance patterns. First-generation cephalosporins are the agents most commonly studied and used for antimicrobial prophylaxis in joint replacement procedures.

Clindamycin and vancomycin have adequate activity against the most common pathogens involved in orthopedic procedures and would be acceptable alternatives under certain circumstances, such as prophylaxis for patients with a $\beta$-lactam allergy. Vancomycin should be included with cefazolin or used as an alternative agent for routine antimicrobial prophylaxis in institutions that have a high prevalence of MRSA SSIs and for patients who are known to be colonized with MRSA [6,8,41,733,781]. Readers are referred to the section on implantation of internal fixation devices for further discussion of antimicrobial prophylaxis choice.

Antimicrobial-laden bone cement. The use of antimicrobial-laden bone cement in conjunction with i.v. antimicrobial prophylaxis is common worldwide, particularly for the prevention of infection in primary hip and knee arthroplasties [802-806]. The FDA has approved premixed aminoglycoside (i.e., gentamicin and tobramycin) in bone cement products for use in hip, knee, or other joints in second-stage revision of total joint arthroplasty [807]. The products are not approved for prophylaxis in primary joint replacement procedures. While antimicrobial bone cement has not been shown to be superior to i.v. antimicrobials $[808,809]$, there is evidence that supports the combination of using antimicrobial-laden bone cement together with systemic antimicrobial prophylaxis.

Although the evidence for the prophylactic use of antimicrobial-laden bone cement in primary joint arthroplasty looks favorable, a recent multicenter evaluation of risk factors for SSI in patients undergoing total hip arthroplasty did not find that use of antimicrobial-laden bone cement reduced the risk for infection [95]. In addition, questions remain regarding the risk for antimicrobial resistance and allergy, as well as the increased cost $[41,802-807,810-813]$. Readers are referred to reviews of this topic for additional information about tissue penetration, clinical application, and safety [805,810-815]. 
Duration. The duration of prophylaxis in joint replacement procedures has been controversial. More recent data and clinical practice guidelines do not support prophylaxis beyond $24 \mathrm{~h}[6,41,133,723]$. Studies involving total hip replacement have used antimicrobials for $12 \mathrm{~h}$ to 14 days postoperatively [726,734-737,816]. A duration of $24 \mathrm{~h}$ was supported in a randomized trial of 358 patients undergoing total hip arthroplasty, total knee arthroplasty, or hip fracture repair that compared prophylaxis that lasted $24 \mathrm{~h}$ versus $7 \mathrm{~d}$ of either nafcillin or cefazolin started $20 \mathrm{~min}$ before the procedure [816]. The difference in SSI rates between groups was not significant. There is no evidence of benefit of antimicrobial administration until all drains or catheters are removed [32,41,133].

\section{Recommendations}

The recommended regimen for patients undergoing total hip, elbow, knee, ankle, or shoulder replacement is cefazolin. Clindamycin and vancomycin should be reserved as alternative agents, as described in the Common Principles section. If there are any surveillance data showing that gram-negative organisms are a cause of SSIs for the procedure, practitioners may consider combining clindamycin or vancomycin with another agent (cefazolin if the patient is not $\beta$-lactam allergic; aztreonam, gentamicin, or a single-dose fluoroquinolone if the patient is $\beta$-lactam allergic). Mupirocin should be given intranasally to all patients with documented colonization with S. aureus. (Strength of evidence for prophylaxis $=$ A.)

\section{Urologic procedures}

\section{Background}

The goals of antimicrobial prophylaxis in urologic procedures are the prevention of bacteremia and SSIs and the prevention of postoperative bacteriuria [59]. Postoperative urinary tract infections (UTIs) are the main concern for morbidity in patients after urologic procedures $[817,818]$. Bacteriuria, defined as $>10^{3}$ or $>10^{4} \mathrm{CFU} / \mathrm{mL}$ in symptomatic UTI and $>10^{5} \mathrm{CFU} / \mathrm{mL}$ in asymptomatic bacteriuria, within $30 \mathrm{~d}$ postoperatively is a frequent primary outcome in urologic procedure studies [819-825]. The benefits of preventing postoperative bacteriuria are not clearly known [825].

In addition to general risk factors discussed in the Common Principles section of these guidelines, urologic-specific risk factors include anatomic anomalies of the urinary tract, [818] urinary obstruction, [826] urinary stone, [817,825,826] and indwelling or externalized catheters [817,818,822,826]. Preoperative UTI, particularly if recurrent, is recognized as a highrisk factor for postoperative infection, which is typically treated before procedures and is a common exclusion criterion from studies of efficacy of antimicrobial prophylaxis in urologic procedures [817,826-828]. Additional urologic operationspecific risk factors include length of postoperative catheterization, [829] mode of irrigation (closed versus open), and postoperative pyuria [821].

\section{Organisms}

Escherichia coli is the organism most commonly isolated in patients with postoperative bacteriuria; however, other gramnegative bacilli and enterococci may also cause infection [818,821,827,830-839]. Organisms such as S. aureus, coagulasenegative Staphylococcus species, and group A Streptococcus species are also a concern in procedures entering the skin with or without entering the urinary tract $[818,827,830-832,838$, 840,841]. There is also some concern with biofilm-forming bacteria (S. epidermidis and $P$. aeruginosa) in patients with prosthesis implantation [842].

\section{Efficacy}

The efficacy of antimicrobial prophylaxis in select urologic procedures has been investigated in several clinical trials. Of note, many of these placebo-controlled studies have excluded patients with risk factors for infection, those requiring antimicrobial prophylaxis for another indication (e.g., infective endocarditis), and those with preoperative UTI or bacteriuria.

The efficacy of antimicrobial prophylaxis in clean procedures among patients at low risk of complications has been variable. One randomized, placebo-controlled study of oral antimicrobials in 2,083 patients undergoing flexible cystoscopy found a positive urine culture (bacteriuria with $>10^{5}$ $\mathrm{CFU} / \mathrm{mL}$ ) in $9.1 \%$ of patients receiving placebo, $4.6 \%$ of patients receiving trimethoprim, and $2.8 \%$ of patients receiving ciprofloxacin [839]. The rates of bacteriuria compared with baseline were significantly higher with placebo and significantly lower with use of antimicrobials compared with placebo. A randomized, placebo-controlled study of 517 patients undergoing prostate brachytherapy found no significant difference in postimplantation epididymitis with or without antimicrobial prophylaxis ( $0.4 \%$ and $1.5 \%$, respectively) [843]. A meta-analysis of eight randomized, placebo-controlled or no-treatment-controlled studies with 995 patients undergoing urodynamic studies found a decrease in bacteriuria with antimicrobial prophylaxis (OR, 0.39; 95\% CI, 0.24-0.61) [820]. The number needed to treat was 13 to prevent one episode of asymptomatic bacteriuria using a pooled rate of $13.7 \%$ for bacteriuria. One study found that not using antimicrobial prophylaxis was a significant risk factor for bacteriuria caused by urinary dynamic studies [821].

Antimicrobial prophylaxis has been studied in urologic procedures involving entry into the gastrointestinal tract, with the majority of the literature on transurethral resection of the prostate (TURP) and prostate biopsy. Two large metaanalyses have suggested prophylactic antimicrobials may be effective in all patients undergoing TURP, including low-risk patients and those with preoperatively sterile urine [844,845]. One meta-analysis of 32 trials with 4,260 patients found that prophylactic antimicrobials decreased the combined bacteriuria ( $>10^{5} \mathrm{CFU} / \mathrm{mL}$ ) event rate from $26 \%$ to $9.1 \%$, for a relative risk reduction of $65 \%(95 \% \mathrm{CI},-55$ to -72$)$, and the combined clinical septicemia episode rate from $4.4 \%$ to $0.7 \%$ in TURP patients, including low-risk patients [846]. Another metaanalysis of 28 trials that included a total of 4,694 patients found prophylactic antimicrobials decreased the post-TURP rate of bacteriuria, fever, and bacteremia, as well as the need for additional postoperative antimicrobials [847]. An additional multicenter, open-label, randomized, active- and placebo-controlled trial in patients with sterile urine undergoing TURP found a decreased rate of bacteriuria $(\geq 5 \mathrm{CFU} / \mathrm{mL})$ with antimicrobial prophylaxis (21\% with levofloxacin and $20 \%$ with sulfamethoxazole- trimethoprim) compared with placebo $(30 \%)(p=0.009)$ [822].

Three randomized, placebo-controlled studies of patients undergoing transrectal needle biopsy of the prostate found 
significant differences in infectious complications (including bacteriuria, positive urine cultures, and UTI) in patients treated with single doses of oral antimicrobial prophylaxis compared with placebo $[819,837,838]$. These three studies support the routine use of antimicrobial prophylaxis in all patients undergoing transrectal needle biopsy of the prostate. Of note, all patients undergoing transrectal needle biopsy of the prostate received a cleansing enema before the procedure $[819,837,838]$. Use of MBP has been reported in urologic procedures that involve entering the gastrointestinal tract (e.g., urinary diversion) $[844,846]$.

The use of antimicrobial prophylaxis in patients undergoing extracorporeal shock wave lithotripsy (ESWL) and ureterorenoscopy is supported by the results of a meta-analysis [847] and a small randomized controlled trial [848]. The metaanalysis included eight randomized controlled trials with 885 patients and six clinical case series involving 597 patients undergoing ESWL [845]. The overall rate of UTI in the randomized controlled trials ranged from $0 \%$ to $7.7 \%$ with antimicrobial prophylaxis and from $0 \%$ to $28 \%$ in the control groups (relative risk, $0.45 ; 95 \% \mathrm{CI}, 0.22-0.93$ ). A randomized, placebo-controlled study of 113 patients undergoing ureterorenoscopy found a rate of postoperative bacteriuria of $1.8 \%$ with antimicrobial prophylaxis and $12.5 \%$ without $(p=0.0026)$ [848]. No patients had symptomatic UTI or inflammation complications of the urogenital tract postoperatively. There are no studies of antimicrobial prophylaxis in major open or laparoscopic procedures (cystectomy, radical prostatectomy, and nephrectomy); therefore, data have been extrapolated from other major intraabdominal procedures.

Choice of agent. No single antimicrobial regimen appears superior for urologic procedures. A wide range of antimicrobial regimens, including cephalosporins $[658,835,836,843,849$ 855], aminoglycosides [856,857], piperacillin-tazobactam $[849,858,859]$, trimethoprim-sulfamethoxazole [822,838,860], trimethoprim [839], nitrofurantoin [861], and fluoroquinolones $[819,821,822,824,831,835-837,839,840,843,848,851,853-855,862$, $863]$, have been evaluated in urologic procedures. The efficacy of fluoroquinolones for antimicrobial prophylaxis in urologic surgical procedures has been well established. One study found better reduction of bacteriuria with either ciprofloxacin or trimethoprim compared with placebo [839], while other studies found no difference in efficacy between a fluoroquinolone and sulfamethoxazole-trimethoprim, both of which were better than placebo $[822,838]$. No differences were found in studies between oral or i.v. fluoroquinolones (ciprofloxacin or ofloxacin) compared with i.v. or intramuscular cephalosporins (ceftriaxone, cefotaxime, or cefazolin) and intramuscular penicillin (piperacillin-tazobactam) in various urologic procedures $[835,836,851,854,855,858]$. In several studies, fluoroquinolones were administered orally, which appears to be feasible in patients undergoing procedures not involving opening the urinary or gastrointestinal tract, when the i.v. route would be preferred $[822,836,838,851,855,858]$. Recently, resistance to fluoroquinolones has been emerging; the fact that most of the literature was published before resistance became prevalent should be considered, since resistance may decrease the relevance of these studies [836,846,847, 858,864]. Local resistance patterns to fluoroquinolones, particularly with $E$. coli, should be evaluated to help guide antimicrobial selection.
Broad-spectrum antimicrobials, such as third-generation cephalosporins and carbapenems, are no more effective than first- or second-generation cephalosporins, aminoglycosides, or oral agents (trimethoprim-sulfamethoxazole, nitrofurantoin, or fluoroquinolones) and should be reserved for patients with active infection or who require additional coverage for intestinal organisms. [6,826,827] Their routine use is not recommended due to their higher cost and potential to promote resistance, particularly among health-care-associated gram-negative bacilli [8].

Duration. While longer durations of postoperative prophylaxis (up to three weeks) have been studied [856,858, $860,861]$, more-recent data support the use of shorter durations (i.e., a single dose or less than $24 \mathrm{~h}$ duration) in urologic procedures $[658,817,818,823,824,826,831,832,834,836,846,853$, $857,859,862,865,866]$. Based on bioavailability, oral antimicrobial prophylaxis should be administered $1-2 \mathrm{~h}$ before surgical incision or start of the procedure $[817,819-822,824,826$, $836,838,840,848,851,855]$.

\section{Pediatric efficacy}

Limited data on antimicrobial prophylaxis are available for pediatric patients undergoing urologic procedures. One prospective, open-label, nonrandomized study of boys undergoing hypospadias repair with tabularized incision plate urethroplasty allocated patients to receive cefonicid (no longer available in the United States) with one i.v. dose before the procedure only or the addition of oral cephalexin three times daily starting on postoperative day 1 until two days after catheter removal (median, 8.3 days) [833]. More patients in the single-dose group had bacteriuria and complications (urethrocutaneous fistula and meatal stenosis); however, the rate of infection and infection-related complications did not significantly differ between groups.

\section{Recommendations}

No antimicrobial prophylaxis is recommended for clean urologic procedures in patients without risk factors for postoperative infections. Patients with preoperative bacteriuria or UTI should be treated before the procedure, when possible, to reduce the risk of postoperative infection. For patients undergoing lower urinary tract instrumentation with risk factors for infection, the use of a fluoroquinolone or trimethoprimsulfamethoxazole (oral or i.v.) or cefazolin (i.v. or intramuscular) is recommended (Table 2). For patients undergoing clean urologic procedures without entry into the urinary tract, cefazolin is recommended, with vancomycin or clindamycin as an alternative for those patients allergic to $\beta$-lactam antimicrobials. For patients undergoing clean urologic procedures with entry into the urinary tract, cefazolin is recommended, with alternative antimicrobials to include a fluoroquinolone, the combination of an aminoglycoside plus metronidazole, or an aminoglycoside plus clindamycin. For clean-contaminated procedures of the urinary tract (often entering the gastrointestinal tract), antimicrobials as recommended for elective colorectal surgery are recommended. This would generally include the combination of cefazolin with or without metronidazole, cefoxitin, or, for patients with $\beta$-lactam allergy, a combination of either a fluoroquinolone or aminoglycoside given with either metronidazole or 
clindamycin. The medical literature does not support continuing antimicrobial prophylaxis until urinary catheters have been removed. See the colorectal procedures section of these guidelines for recommendations pertaining to procedures entering the gastrointestinal tract. (Strength of evidence for prophylaxis =A.)

\section{Vascular procedures}

\section{Background}

Infection after vascular procedures occurs with low frequency but can be associated with extensive morbidity and mortality $[867,868]$. Postoperative infections involving vascular graft material can result in limb loss and life-threatening conditions [868]. As a result, antimicrobial prophylaxis is widely used in procedures that involve implantation of prosthetic material and procedures for which there is greater risk of infection, such as aneurysm repair, thromboendarterectomy, and vein bypass $[6,41,867,869]$. Patients undergoing brachiocephalic procedures (e.g., carotid endarterectomy, brachial artery repair) without implantation of prosthetic graft material do not appear to benefit from routine antimicrobial prophylaxis $[6,41,867,870]$.

Risk factors for postoperative SSI in patients undergoing vascular procedures include lower-extremity sites, delayed procedures after hospitalization, diabetes mellitus, and a history of vascular or aortocoronary bypass procedures [871,872]. Currently, prospective data from well-designed studies on prophylaxis for endovascular stenting do not exist. However, if prophylaxis is desired, the same antimicrobials and short duration of therapy used for open vascular procedures should be given. Risk factors that warrant consideration of prophylaxis in patients undergoing endovascular stenting include prolonged procedures (more than two hours), reintervention at the surgical site within seven days, vascular stent placement in the groin through a hematoma or sheath, procedures in immunosuppressed patients, and the presence of another intravascular prosthesis [873-877].

\section{Organisms}

The predominant organisms involved include S. aureus, S. epidermidis, and enteric gram-negative bacilli. MRSA is an emerging organism of concern. Several studies evaluated the rate of colonization, carriage, and infection with MRSA in patients undergoing various vascular procedures [878-884]. Independent risk factors for MRSA infection included MRSA colonization, open abdominal aortic aneurysm, tissue loss, and lower-limb bypass [878]. Patients who have or develop MRSA infections before vascular procedures have increased risk of inhospital death, intensive care unit admission, repeat surgeries, increased length of stay, and delayed wound healing, compared with patients without infections [880-883].

\section{Efficacy}

Prophylactic antimicrobials decrease the rate of infection after procedures involving the lower abdominal vasculature and procedures required to establish dialysis access. The follow-up time for patients with late surgical site complications was at least once after hospital discharge (not further defined) for most studies [829,865,871,885-887], at one month $[869,871,888,889]$, at six months [872], and at three years [138].
A meta-analysis of 10 randomized controlled trials in patients undergoing peripheral arterial reconstruction with biological or prosthetic graft procedures found an overall consistent reduction in SSIs with systemic antimicrobial prophylaxis compared with placebo (relative risk, 0.25 ; $95 \% \mathrm{CI}$, 0.17-0.38; $\mathrm{p}<0.00001$ ) [890]. An overall reduction was found among 5 studies evaluating early graft infection (relative risk, $0.31 ; 95 \% \mathrm{CI}, 0.11-0.85 ; \mathrm{p}=0.02)$, though no individual study found a significant reduction in SSIs.

The largest study included in the meta-analysis above was a randomized, prospective, double-blind, placebo-controlled study of patients undergoing peripheral vascular procedures $(n=462)$. The infection rate was significantly lower with cefazolin than with placebo $(0.9 \%$ and $6.8 \%$, respectively) [885]. Four deep graft infections were observed in the placebo group; none occurred in the patients who received cefazolin. No infections were observed in patients who underwent brachiocephalic $(n=103)$, femoral artery $(n=56)$, or popliteal $(n=14)$ procedures.

Patients undergoing vascular access procedures for hemodialysis may benefit from the administration of antistaphylococcal antimicrobials. A placebo-controlled study of 408 patients undergoing permanent vascular access placement demonstrated an upper-extremity prosthetic polytetrafluoroethylene graft infection rate of $6 \%$ with placebo compared with $1 \%$ with vancomycin $(p=0.006)$. [869]

Choice of agent. Cefazolin remains the preferred and most cost-effective prophylactic agent for use in vascular procedures $[6,8,41,872,886,887]$. There was no significant difference in infection rates between cefazolin and cefuroxime in patients undergoing abdominal aortic and lower-extremity peripheral vascular procedures, [886] between cefazolin and cefamandole (no longer available in the United States) in patients undergoing aortic or infrainguinal arterial procedures, [887] or between cefazolin and ceftriaxone in patients undergoing arterial reconstruction involving infraclavicular sites [872].

A multicenter, randomized, double-blind, prospective trial of 580 patients undergoing arterial procedures involving the groin who received either two doses of ciprofloxacin $750 \mathrm{mg}$ orally or three doses of cefuroxime $1.5 \mathrm{~g}$ i.v. on the day of the procedure found an SSI rate of 9.2\% (27 patients) and 9.1\% (26 patients), respectively, within $30 \mathrm{~d}$ of the procedure [889] Although oral ciprofloxacin was shown to be as effective as i.v. cefuroxime, this study did not address concerns about resistance with routine use of fluoroquinolones [891]. Therefore, i.v. cefazolin remains the first-line agent for this indication. The efficacy of oral agents for prophylaxis needs to be further evaluated.

There are limited data regarding the choice of an antimicrobial for $\beta$-lactam-allergic patients undergoing vascular procedures. The main alternative agents are vancomycin and clindamycin, since prophylaxis is largely directed against gram-positive cocci. Vancomycin can also be used for prophylaxis in institutions with MRSA or methicillin-resistant S. epidermidis (MRSE) clusters or in patients with $\beta$-lactam allergy $[6,8,41]$. Clindamycin may be an acceptable alternative to vancomycin, though local antimicrobial resistance patterns should be taken into account.

An aminoglycoside may be added to vancomycin for the addition of aerobic gram-negative bacilli coverage if the 
procedure involves the abdominal aorta or a groin incision, due to the potential for gastrointestinal flora. See the Common Principles section of these guidelines for further discussion of the use of vancomycin. Alternative antimicrobials for $\beta$-lactam-allergic patients receiving vancomycin may include a fluoroquinolone or aztreonam [6].

Duration. A meta-analysis of three randomized controlled studies involving vascular procedures, including lower-limb reconstruction and open arterial procedures, found no additional benefit of continuing prophylactic antimicrobials for over $24 \mathrm{~h}$ postoperatively compared with no more than $24 \mathrm{~h}$ (relative risk, 1.28; 95\% CI, 0.82-1.98) [890].

A randomized, double-blind study compared infection rates of a one-day and a three-day course of cefuroxime with placebo in 187 patients undergoing peripheral vascular procedures [888]. The infection rates were $16.7 \%, 3.8 \%$, and $4.3 \%$ in the placebo, one-day, and three-day groups, respectively. The difference in the infection rates between the oneand three-day groups was not significant.

A randomized controlled study compared one day and five days of amoxicillin-clavulanate $1.2 \mathrm{~g}$ in 100 patients undergoing 108 lower-limb reconstruction procedures [892]. No difference was seen in the postoperative SSI rate between groups (9 patients [16\%] and 12 patients [23\%], respectively). The study authors selected the agent based on extended spectrum of activity and good tissue penetration. However, they concluded that due to the high rate of infection observed, the use of antimicrobial prophylaxis might not be as effective as once thought.

A randomized controlled study compared ticarcillinclavulanate $3.1 \mathrm{~g}$ given as a single dose at induction of anesthesia with multiple doses given at induction and every $6 \mathrm{~h}$ postoperatively until venous access lines were removed or a maximum of 20 doses (total of five days) in patients undergoing open arterial procedures. [893] Significantly more SSIs occurred in the single-dose group (28 [18\%] of 153 patients) compared with the multidose group (15 [10\%] of 149 patients) (relative risk, 2.00; 95\% CI, -1.02 to $3.92 ; \mathrm{p}=0.041$ ). Ticarcillin-clavulanate has a short duration of action and is not recommended as a routine agent for antimicrobial prophylaxis. Practice guidelines recommend single-dose prophylaxis in vascular procedures or a maximum duration of therapy of $24 \mathrm{~h}$ postoperatively, regardless of the presence of invasive drains $[6,41]$.

\section{Recommendations}

The recommended regimen for patients undergoing vascular procedures associated with a higher risk of infection, including implantation of prosthetic material, is cefazolin (Table 2). (Strength of evidence for prophylaxis=A.) Clindamycin and vancomycin should be reserved as alternative agents as described in the Common Principles section of these guidelines. If there are surveillance data showing that gramnegative organisms are a cause of SSIs for the procedure, practitioners may consider combining clindamycin or vancomycin with another agent (cefazolin if the patient is not $\beta$-lactam-allergic; aztreonam, gentamicin, or single-dose fluoroquinolone if the patient is $\beta$-lactam-allergic), due to the potential for gastrointestinal flora exposure.

\section{Heart, lung, and heart-lung transplantation}

\section{Background}

Solid-organ transplant recipients are at high risk for infections due to the complexity of the surgical procedures, donor- or recipient-derived infections, reactivation of recipientassociated latent infections, preoperative recipient colonization, exposure to community pathogens, and opportunistic infections due to immunosuppression [894-897]. Infections occur more frequently in the first year after transplantation, due to aggressive immunosuppression. Transplant recipients with infections are commonly asymptomatic or have nonspecific symptoms or sequelae of infection, which makes detection and diagnosis of infections difficult [855,857,894]. Postoperative infections caused by bacterial, viral, and fungal pathogens, including SSIs, UTIs, blood stream infections, and pneumonia, are of greater concern within the first month after transplantation [895-897]. Opportunistic infections that result from immunosuppression typically occur after the first month of transplantation. It is routine for transplant recipients to receive antimicrobial prophylaxis to prevent opportunistic infections [894-897]. A discussion of the prophylactic strategies for prevention of cytomegalovirus (CMV) infection, herpes simplex virus infection, pneumocystis, UTI in kidney transplant recipients, Aspergillus infection in lung transplant recipients, and other opportunistic infections outside of the immediate posttransplantation period is beyond the scope of these guidelines.

Few well-designed, prospective, comparative studies of antimicrobial prophylaxis have been conducted with patients undergoing solid-organ transplantation, and no formal recommendations are available from expert consensus panels or professional organizations; however, there are reviews that provide guidance $[8,41,894]$.

The recommendations given for each of the solid-organ transplant procedures are intended to provide guidelines for safe and effective surgical prophylaxis based on the best available literature. Antimicrobial surgical prophylaxis practice will vary considerably among transplantation centers throughout the United States, based on the organ involved, preexisting recipient and donor infections, and local antimicrobial susceptibilities [894-897].

\section{Heart transplantation}

Background. Heart transplantation is an option for selected patients with end-stage cardiac disease. In 2007, the United Network for Organ Sharing (UNOS) reported that 2,209 heart transplants were performed in the United States, including 327 in children ( $<18$ years of age) [898]. The mean graft survival rate 10 years after heart transplantation is approximately $49 \%$. Infection continues to be an important cause of morbidity and mortality after heart transplantation and is a primary cause of death in approximately $14 \%$ of patients within the first year after transplantation [899].

Despite the large number of heart transplantation procedures performed, few studies have specifically examined postoperative SSI rates in this population. General cardiothoracic procedures have been associated with SSI rates ranging from $9 \%$ to $55 \%$ in the absence of antimicrobial prophylaxis [214,900,901]. Studies of general cardiothoracic procedures, including heart transplantation, found SSIs, particularly mediastinitis, in $3-6 \%$ of patients who received 
antimicrobial prophylaxis [170,902]. The frequency was highest in heart transplant recipients. The SSI rates reported in patients undergoing heart transplantation who received antimicrobial prophylaxis ranged from $5.8 \%$ to $8.8 \%$, including mediastinitis in $3 \%-7 \%$ of patients $[903,904]$.

Several independent risk factors for SSIs after cardiac and thoracic procedures have been identified (see the cardiac and thoracic sections of this article). Heart transplantation has been identified as an independent risk factor for SSIs [170]. Other independent risk factors for SSIs in heart transplantation include age [905], receipt of ciprofloxacin alone for prophylaxis [906], positive wire cultures [907], a BMI of $>30 \mathrm{~kg} / \mathrm{m}^{2}$, female sex [908], previous cardiac procedures, previous left VAD placement, and hemodynamic instability requiring inotropic support $[903,904]$. Unfavorable functional outcomes were seen in patients who developed infections within the first year after heart transplantation associated with lung, bloodstream, and CMV infections [909]. Independent predictors of mortality in heart transplant recipients included serum creatinine levels, amyloid etiology, history of hypertension, pulmonary infection, and CNS infection. Additional predisposing factors for infection in heart transplantation include exposure to pathogens from the donor or transplant recipient, the time from organ recovery to reperfusion, and the immunosuppressive regimens used $[897,904,910]$. Similar risk factors for infection are noted in pediatric transplant recipients, with the addition of a naive immune system to several pathogens, most notably viruses, as well as incomplete primary immunization series [897].

Patients with an indwelling VAD at the time of heart transplantation have additional prophylaxis concerns. Recipients who do not have a driveline infection and have no history of either colonization or infection should receive prophylaxis as described for recipients without a VAD in place. Patients with a history of colonization or previous infection should have the antimicrobial sensitivities of that organism considered when choosing the SSI prophylactic regimen administered, though the duration should still be less than $24 \mathrm{~h}$. Heart transplant recipients with an active VAD driveline infection at the time of heart transplantation should be given appropriate antimicrobials specifically for the treatment of that infection. This intervention will usually determine the actual perioperative prophylaxis regimen as well as the duration of therapy beyond the period of prophylaxis.

Patients requiring ECMO as a bridge to heart transplantation should be treated with a similar approach. If there is no history of colonization or previous infection, then the general recommendations for SSI antimicrobial prophylaxis for the specific procedure should be followed. In ECMO patients with a history of colonization or previous infection, changing the preoperative antimicrobial prophylaxis to cover these pathogens must be considered, weighing whether the pathogen is relevant to SSIs in the planned procedure.

Because heart transplantation is similar to other cardiac and thoracic procedures, similar considerations regarding the need for antimicrobial prophylaxis apply (see the cardiac and thoracic sections) [911]. These guidelines do not address antimicrobial prophylaxis for infective endocarditis. Readers are referred to the current guidelines for prevention of infective endocarditis from AHA $[11,228]$.

Organisms. As with other types of cardiothoracic procedures, gram-positive organisms, mainly Staphylococcus species, are the primary pathogens that cause SSI after heart transplantation [902,905-907,912,913]. Methicillinresistant S. aureus was reported in $12 \%-21 \%$ of SSIs in several cohort studies $[903,905,906]$. Vancomycin-resistant E. faecalis was noted in $15 \%$ of infections in one cohort study [903]. Other gram-positive pathogens (e.g., coagulase-negative staphylococci, Enterococcus species) [903,905-907,913] and gramnegative organisms (e.g., Enterobacteriaceae, P. aeruginosa, Stenotrophomonas maltophilia) are also a concern for SSIs in heart transplant recipients, as are Candida species [903,906].

Efficacy. Despite the paucity of literature on antimicrobial prophylaxis for the prevention of SSIs in heart transplantation, the efficacy noted in other cardiac surgical procedures has made it the standard of practice during transplantation [896].

No randomized controlled trials have specifically addressed the use of antimicrobial prophylaxis in heart transplantation. In an open-label noncomparative study, the SSI rate was $4.5 \%$ among 96 patients administered cefotaxime plus floxacillin preoperatively and for $72 \mathrm{~h}$ after cardiac procedures [912]. This rate of infection was similar to that seen in other cardiothoracic, nonheart transplantation procedures in which antimicrobial prophylaxis was used.

Choice of agent. Antimicrobial prophylaxis for heart transplantation should be similar to that used for other types of cardiothoracic procedures [911]. First- and secondgeneration cephalosporins are considered to be equally efficacious and are the preferred agents. There appear to be no significant differences in efficacy among prophylactic regimens using agents such as cefazolin and cefuroxime [914]. The use of antistaphylococcal penicillins, either alone or in combination with aminoglycosides or cephalosporins, failed to demonstrate superior efficacy to that of cephalosporin monotherapy (see the cardiac and thoracic sections) in other cardiothoracic procedures.

Several cohort studies examined antimicrobial prophylactic agents used for patients undergoing heart transplantation but did not evaluate efficacy $[902,903,905,906]$. Ciprofloxacin alone was found to be an independent risk factor for incisional SSI [906].

Duration. There is no consensus on the optimal duration of antimicrobial prophylaxis in cardiothoracic procedures, including heart transplantation. Cohort evaluations of patients undergoing heart transplantation reported durations of antimicrobial prophylaxis with cefazolin or vancomycin of 24 or $48 \mathrm{~h}$ postoperatively $[902,903,905]$. Data from cardiothoracic procedures also support a range of prophylaxis durations, from a single dose to 24 or $48 \mathrm{~h}$ postoperatively $[41,131]$. The currently accepted duration for these procedures, which do not include transplantation, is $24-48 \mathrm{~h}$ postoperatively $[41,59,131,201]$. The duration of antimicrobial prophylaxis for patients who do not have their chest primarily closed is unclear; most centers continue prophylaxis until the chest is closed, but there is no evidence to support this practice.

Pediatric efficacy. No randomized controlled studies have specifically addressed antimicrobial prophylaxis for heart transplantation in pediatric patients. Infants are at risk 
for mediastinitis caused by gram-negative as well as grampositive organisms. Pediatric patients undergoing heart transplantation should be treated according to recommendations for other types of cardiothoracic procedures. The recommended regimen for pediatric patients undergoing cardiothoracic procedures is cefazolin $25-50 \mathrm{mg} / \mathrm{kg}$ i.v. within $60 \mathrm{~min}$ before surgical incision and every $8 \mathrm{~h}$ for up to $48 \mathrm{~h}$. Cefuroxime $50 \mathrm{mg} / \mathrm{kg}$ i.v. within 60 minutes before surgical incision and every $8 \mathrm{~h}$ for up to 48 hours is an acceptable alternative. Vancomycin $10-20 \mathrm{mg} / \mathrm{kg}$ i.v. over 60 $120 \mathrm{~min}$, with or without gentamicin $2 \mathrm{mg} / \mathrm{kg}$ i.v., should be reserved as an alternative on the basis of guidelines from HICPAC for routine antimicrobial prophylaxis in institutions that have a high prevalence of MRSA, for patients who are colonized with MRSA, or for patients with a true $\beta$-lactam allergy [8]. Additional doses may be needed intraoperatively for procedures $>4 \mathrm{~h}$ in duration, for patients with major blood loss, or for extended use of CPB depending on the halflife of the prophylactic antimicrobial. Fluoroquinolones are not routinely recommended in pediatric patients.

Recommendations. Based on data for other types of cardiothoracic procedures, antimicrobial prophylaxis is indicated for all patients undergoing heart transplantation (see cardiac and thoracic sections). The recommended regimen is a single dose of cefazolin (Table 2). There is no evidence to support continuing prophylaxis until chest and mediastinal drainage tubes are removed. Alternatives include vancomycin or clindamycin with or without gentamicin, aztreonam, or a single fluoroquinolone dose. (Strength of evidence for prophylaxis=A.) The optimal duration of antimicrobial prophylaxis for patients who do not have their chest primarily closed is unclear. No recommendation is made for these patients. Patients who have left VADs as a bridge and who are chronically infected might also benefit from coverage of the infecting microorganism.

\section{Lung and heart-lung transplantation}

Background. Lung transplantation is an accepted option for a variety of end-stage, irreversible lung diseases. The most common diseases for which lung transplantation is performed are idiopathic pulmonary fibrosis, chronic obstructive pulmonary disease, emphysema, cystic fibrosis, $\alpha$-1-antitrypsin deficiency, and idiopathic pulmonary arterial hypertension $[915,916]$. The UNOS reported that in the United States in 2007, 1,468 lung transplantations and three heart-lung transplantations were conducted in adults, and 52 lung transplantations and three heart-lung transplantations were performed in children [898,917]. Ten-year survival rates were reported as $29.7 \%$ of double-lung, $17.5 \%$ of single-lung, and $25.8 \%$ of heart-lung transplant recipients [899]. The reported three-year survival rate for pediatric lung transplant recipients was 57\% [897].

Infections are the most common complications after lung and heart-lung transplantations [899,915,918,919]. In an analysis of UNOS data over an 18-year period, infection was the number one cause of death within the first year of transplantation, occurring in $24.8 \%$ of lung and $18.3 \%$ of heartlung transplant recipients [899]. Among the top 10 primary causes of death within the first year after lung and heart-lung transplantations were sepsis, pneumonia, fungal infection (lung only), and CMV infection [899]. A study of two cohorts of patients undergoing heart, lung, and heart-lung transplantations who received antimicrobial prophylaxis evaluated the rate of SSIs and mediastinitis [904,908]. The rate of SSI among all transplant recipients was $12.98 \%$, with the majority of infections ( $72 \%$ ) being organ/space infections, followed by deep incisional infections (17\%) and superficial incisional infections (10\%) [908]. The overall rate of mediastinitis in a similar cohort was $2.7 \%$, with rates of $5.2 \%$ in heart-lung transplant recipients and $3.2 \%$ in bilateral lung transplant recipients [904]. Pneumonia was reported in $26.4 \%$ of transplantation patients overall, with rates of $20.7 \%$ in lung transplant recipients and $40 \%$ in heart-lung transplant recipients [908]. A cohort of lung transplant recipients reported a rate of 2.2 episodes of pneumonia per patient during a median followup period of 412 days (range, 1-1,328 days) [920].

Bronchial anastomotic infections, especially fungal infections, can be serious and are potentially fatal in lung transplant recipients [921,922]. The lung allocation score (LAS) is a rating system adopted by the Organ Procurement and Transplant Network and UNOS in 2005 to improve organ allocation and transplantation outcomes. The LAS is based on the risk of death while on the waiting list for transplantation and the expected one-year survival after transplantation. Patients with a low LAS are unlikely to undergo transplantation. A study of lung transplant recipients age 12 years or older revealed a higher rate of infection and other morbidities and a lower one-year survival rate in patients with a high LAS at the time of transplantation than in patients with a low LAS at the time of transplantation [923]. Thus, the potential for bronchial anastomotic infection and a poor posttransplantation outcome needs to be considered in patients undergoing lung transplantation. Among lung transplantation patients, risk factors for nosocomial infections included $\alpha$-1-antitrypsin deficiency and repeat transplantation. Risk factors for pneumonia included colonized or infected donor bronchus and perfusate and preoperative colonization with gram-negative rods. Risk factors for mortality among the transplant recipients were cystic fibrosis, nosocomial infection, and ventilation before transplantation [908]. Risk factors for mediastinitis after heart, lung, and heart-lung transplantation were degree of immunosuppression, impaired renal function, previous sternotomy, and reexploration due to bleeding [904]. There was a positive association between pretransplantation colonizing microorganisms from suppurative lung disease patients and pneumonia after transplantation [920]. Transplantation alters the physiological function of lungs, including the impairment of mucociliary clearance and interruption of the cough reflex, leading to a higher risk of pulmonary infections [896].

In patients requiring ECMO as a bridge to lung transplantation who have no history of colonization or previous infection, the general recommendations for SSI antimicrobial prophylaxis for the procedure should be followed. In ECMO patients with a history of colonization or previous infection, changing the preoperative antimicrobial prophylaxis to cover these pathogens must be considered, weighing whether the pathogen is relevant to SSIs in the planned procedure.

Organisms. While gram-positive and gram-negative organisms are of concern in heart transplantation, there is increased concern regarding gram-negative and fungal pathogens in mediastinitis and pneumonia in patients 
undergoing lung transplantation [894,904,908]. The most frequent organisms found in SSIs or mediastinitis in two cohort studies were P. aeruginosa [904,908], Candida species, S. aureus (including MRSA) [908], enterococci, coagulasenegative staphylococci (e.g., S. epidermidis), Burkholderia cepacia [904], E. coli, and Klebsiella species.

Patients undergoing lung transplantation are also at risk for bacterial or fungal pneumonia due to colonization or infection of the lower and upper airways of the donor, recipient, or both [915]. Organisms reported to cause pneumonia in lung transplantation patients include $P$. aeruginosa [894,896,904, 908,920], S. aureus (including MRSA) [894,896,904,908], B. cepacia [896,904,908], Enterobacter species [908], S. maltophilia, Klebsiella species [904,908], S. epidermidis [904], E. coli, Aspergillus species [920], and VRE [894]. Similarly, organisms frequently seen in pediatric lung infections are nonfermenting gram-negative bacteria, such as Pseudomonas species, Stenotrophomonas species, Alcaligenes species, and fungi, including Aspergillus species [897].

The donor lung appears to be a major route of transmission of pathogens; $75 \%-90 \%$ of bronchial washings from donor organs are positive for at least one bacterial organism $[920,924,925]$. Organ recipients may also be the source of infection of the transplanted organ. This is particularly true in patients with cystic fibrosis because of the frequent presence of $P$. aeruginosa in the upper airways and sinuses before transplantation $[896,919]$. These pathogens are often multidrug-resistant, likely due, in large part, to frequent administration of broad-spectrum antimicrobials during the course of the disease. Multidrug-resistant strains of B. cepacia and S. maltophilia may be a problem in cystic fibrosis patients in some transplantation centers $[919,926]$.

Efficacy. Although much has been published about general infectious complications associated with lung transplantation, no randomized controlled trials regarding antimicrobial prophylaxis for lung or heart-lung transplantation have been published; however, antimicrobial prophylaxis is considered standard practice in these patients [896]. Antimicrobial prophylaxis is routinely administered to patients undergoing lung or heart-lung transplantation, with the aim of preventing pneumonia as well as SSIs. The rate of pneumonia within the first two weeks postoperatively has reportedly been decreased from $35 \%$ to approximately $10 \%$ by routine antimicrobial prophylaxis [927-929]. Improvements in surgical technique and postoperative patient care are also important factors in the apparently lower rates of pneumonia after lung transplantation.

Choice of agent. No formal studies have addressed optimal prophylaxis for patients undergoing lung transplantation. Antimicrobial prophylaxis for lung and heart-lung transplantation should generally be similar to that used for other cardiothoracic procedures (see the cardiac and thoracic sections). First- and second-generation cephalosporins are considered equally efficacious and are the preferred agents for these procedures. However, prophylactic regimens should be modified to include coverage for any potential bacterial pathogens, including gram-negative and fungal organisms, that have been isolated from the recipient's airways or the donor lung through preoperative cultures [894,896,904,908, $915,920]$. Patients with end-stage cystic fibrosis should receive antimicrobials on the basis of the known susceptibilities of pretransplant isolates, particularly $P$. aeruginosa, B. cepacia complex, and Aspergillus species.

Antimicrobial prophylaxis regimens reported in cohort evaluations of thoracic transplantation, including lungs, have varied $[904,908,920]$. One study used ceftazidime, floxacillin, tobramycin, and itraconazole in these patients [908]. In addition, all patients received nebulized amphotericin B and oral itraconazole as antifungal prophylaxis. Another cohort study used cefepime for lung transplant recipients without known colonization; for those with known colonization, the selection of agents was based on organism susceptibility [920]. A third cohort reported use of metronidazole and aztreonam as prophylaxis for patients with a septic lung (positive sputum culture) [904].

Antifungal prophylaxis should be considered, especially when pretransplantation cultures reveal fungi in the donor lung [915] or the recipient's airway. There is no consensus on the appropriate antifungal agent for lung transplant recipients $[894,896,930]$. Selection is recommended based on patient risk factors for infection (e.g., cystic fibrosis) and colonization, pretransplantation and posttransplantation cultures, and local fungus epidemiology $[894,896,897,930]$. Because of the serious nature of fungal infections in the early posttransplantation period and the availability of antifungal agents, prophylaxis should be considered when Candida or Aspergillus species are isolated from the donor lung [915] or recipient's airway.

Duration. No well-conducted studies have addressed the optimal duration of antimicrobial prophylaxis for lung or heart-lung transplantation. In the absence of positive cultures from the donor or the recipient, prophylactic regimens of $48-72 \mathrm{~h}$ and no longer than $7 \mathrm{~d}$ have been reported [896,904, $905,931]$. In patients with positive pretransplantation cultures from donor or recipient organs or patients with positive cultures after transplantation, postoperative antimicrobial treatment for 7-14 d or longer has been reported, particularly for patients with cystic fibrosis and previous $P$. aeruginosa and multidrug-resistant infections $[896,915,919]$. Such antimicrobial administration is viewed as treatment and not as surgical prophylaxis. Treatment may include additional antibacterial agents or antifungal agents.

Recommendations. Based on data from other types of cardiothoracic procedures, all adult patients undergoing lung transplantation should receive antimicrobial prophylaxis, because of the high risk of infection. Patients with negative pretransplantation cultures should receive antimicrobial prophylaxis as appropriate for other types of cardiothoracic procedures.

The recommended regimen is a single dose of cefazolin (Table 2). There is no evidence to support continuing prophylaxis until chest and mediastinal drainage tubes are removed. Alternatives include vancomycin with or without gentamicin, aztreonam, and a single fluoroquinolone dose. (Strength of evidence for prophylaxis $=\mathrm{A}$.) The optimal duration of antimicrobial prophylaxis for patients who do not have their chest primarily closed is unclear. No recommendation is made for these patients.

The prophylactic regimen should be modified to provide coverage against any potential pathogens, including gramnegative (e.g., $P$. aeruginosa) and fungal organisms, isolated from the donor lung or the recipient pretransplantation. The 
prophylactic regimen may also include antifungal agents for Candida and Aspergillus species based on patient risk factors for infection (e.g., cystic fibrosis) and colonization, pretransplantation and posttransplantation cultures, and local fungus epidemiology. Patients undergoing lung transplantation for cystic fibrosis should receive treatment for at least seven days with antimicrobials selected according to pretransplantation culture and susceptibility results. (Strength of evidence for prophylaxis $=$ B.)

\section{Liver transplantation}

\section{Background}

Liver transplantation is a lifesaving procedure for many patients with end-stage hepatic disease for whom there are no other medical or surgical options [932,933]. In 2007, UNOS reported that 6,494 liver transplantations were performed in the United States, $96 \%$ of which had a cadaveric donor and $4 \%$ had a living-related donor source. [934] These liver transplantations were performed in 5,889 adults and 605 pediatric ( $<18$ years old) patients. Reported one-year patient survival rates for adults ranged from $76.9 \%$ to $95 \%$ [932,935-938] and from $80 \%$ to $91.7 \%$ for pediatric patients [934,939-942]. Survival at three and five years ranged from $68.5 \%$ to $80.9 \%$ [934] and from $61.6 \%$ to $76.5 \%[932,933]$ in adult patients, respectively. In pediatric patients, three- and five-year survival ranged from $73.2 \%$ to $86 \%$ [897,934,941] and from $69.2 \%$ to $80.1 \%$ [934], respectively. One-year graft survival rates ranged from $74.2 \%$ to $94 \%$ in adults $[934-936,938]$ and from $72.1 \%$ to $86.1 \%$ in pediatric patients [934,941,942]. Graft survival at three and five years ranged from $58.9 \%$ to $75.5 \%$ and from $51.6 \%$ to $70.5 \%$, respectively, in adults and from $62.5 \%$ to $77.6 \%$ and from $68.4 \%$ to $71.4 \%$, respectively, in pediatric patients $[934,941]$. No significant differences were noted in graft or patient survival between cadaveric and living-related donors in adult and pediatric liver transplant recipients [934]. Infection remains a major cause of morbidity and mortality in liver transplant recipients. Infections may occur in $31-83 \%$ of patients within three months of transplantation and are the cause of death in $4-53 \%$ of patients [934,936,940,943-950]. These rates are highly variable and do not seem to have changed despite advances in surgical technique and medical management. SSIs within 30 days after transplantation ranged from $4 \%$ to $48 \%$ with antimicrobial prophylaxis in several cohort and controlled studies [935-938,941,942,948,949,951964]. Superficial SSIs are seen most often within the first two to three weeks postoperatively, whereas organ/space infections and deep infections are seen after three to four weeks.

Liver transplantation is often considered to be the most technically difficult of the solid-organ transplantation procedures. Surgical procedures lasting longer than 8-12 hours have been consistently identified as one of the most important risk factors for early infectious complications, including SSIs, intraabdominal infections, and biliary tract infections $[896,938,939,945,947,957]$. Other important risk factors for infectious complications related to liver transplantation surgery include previous hepatobiliary surgery [896,939,945, $947,952,963]$, previous liver or kidney transplantation $[937,951,952,965]$, and surgical complications such as anastomotic leakage [896,938,939,945,947,951,952]. Patient-related risk factors for infection after liver transplantation include antimicrobial use within three to four months before trans- plantation $[935,954]$, low pretransplantation serum albumin concentration [938,958,963], high pretransplantation serum bilirubin concentration [939,945,947], ascites [938], obesity [963], diabetes, and hemochromatosis [966]. Procedure-related risk factors for infection include transfusion of $>4$ units of red blood cells $[896,951]$, bacterial contamination due to entry into the gastrointestinal tract [963], surgical incision method [963], and use of muromonab-CD3 within the first week after transplantation [938].

\section{Organisms}

The pathogens most commonly associated with early SSIs and intraabdominal infections are those derived from the normal flora of the intestinal lumen and the skin. Aerobic gram-negative bacilli, including E. coli [935,937,939,940, 942,945,947-949,951,967,968], Klebsiella species [933,936,937, 939,940,945,947-949,967-969], Enterobacter species [936,939, 940,942,945,947,952,959,964,967,968], A. baumannii [935-937, 942,951], and Citrobacter species [939,940,945,947,952,959, $967,968]$, are common causes of SSIs and intraabdominal infections and account for up to $65 \%$ of all bacterial pathogens. Infections due to $P$. aeruginosa may also occur but are much less common in the early postoperative period [936,937,939, $940,942,945,947,948,952,959,969]$. Enterococci are particularly common pathogens and may be responsible for $20-46 \%$ of SSIs and intraabdominal infections [894,933,935,937,938, 940,943,945-947,951,952,955,964,965,969]. Staphylococcus aureus (frequently MRSA) and coagulase-negative staphylococci are also common causes of postoperative SSIs [936-938, 940,942,943,945-949,955,957-961,964,965,970,971]. Candida species commonly cause both early and late postoperative infections [933,936,937,940,942,943,945-947,949,951,969].

Several studies have noted increasing concern about antimicrobial resistance based on detection of resistant organisms, including E. coli [935,937], Enterococcus species [933,937,964,965], Enterobacter species [964], Klebsiella species $[933,937]$, coagulase-negative staphylococci $[937,964]$, and $S$. aureus $[937,948,957-961,970]$. General information on antimicrobial resistance is provided in the Common Principles section of these guidelines. Of specific concern to the transplantation community is the emergence of multidrugresistant A. baumannii [972], carbapenem-resistant Enterobacteriaceae $[973,974], K$. pneumoniae carbapenemaseproducing organisms [975], and C. difficile [976-978].

\section{Efficacy}

Although there remains a high rate of infection directly related to the liver transplantation procedure, there are few well-controlled studies concerning optimal antimicrobial prophylaxis. In evaluating the efficacy of prophylactic regimens, it is important to differentiate between early infections (occurring within 14-30 days after surgery) and late infections (occurring more than 30 days after surgery). Infections occurring in the early postoperative period are most commonly associated with biliary, vascular, and abdominal surgeries involved in the transplantation procedure itself and are thus most preventable with prophylactic antimicrobial regimens $[939,940,943,945]$. The frequency of these infections varies from $10 \%$ to $55 \%$ despite antimicrobial prophylaxis $[939,940,943,945,979]$. It is difficult to assess the efficacy of prophylactic regimens in reducing the rate of infection, 
because prophylaxis has been routinely used in light of the complexity of the surgical procedure; therefore, reliable rates of infection in the absence of prophylaxis are not available. No controlled studies have compared prophylaxis with no prophylaxis.

Choice of agent. Antimicrobial prophylaxis should be directed against the pathogens most commonly isolated from early infections (i.e., gram-negative aerobic bacilli, staphylococci, and enterococci). Traditional prophylactic regimens have therefore consisted of a third-generation cephalosporin (usually cefotaxime, because of its antistaphylococcal activity) plus ampicillin $[936,937,943,944,946-948,951,952,954,962$, $965,967,979]$. The use of cefoxitin and ampicillin-sulbactam, cefotaxime and ampicillin-sulbactam and gentamicin [957959], cefuroxime and metronidazole [971], ceftriaxone and metronidazole [980], cefotaxime and metronidazole [953], ceftriaxone and ampicillin [949], ceftizoxime alone [955], cefotaxime and tobramycin [956], cefoxitin alone [960,961], cefazolin alone [951], amoxicillin-clavulanate and gentamicin [970], amoxicillin-clavulanate alone [951], glycopeptides and antipseudomonal penicillin [951], fluoroquinolone and amoxicillin-clavulanate or glycopeptide [951], vancomycin and aztreonam [951,981], and piperacillin-tazobactam [964,970] has also been reported. Alternative prophylaxis regimens for $\beta$-lactam-allergic patients have included cefuroxime and metronidazole [970], clindamycin and gentamicin or aztreonam [948,960-962], ciprofloxacin and metronidazole [970], and vancomycin or ciprofloxacin [936]. Imipenem-cilastatin alone was used in one study for patients with renal failure [956]. The efficacy of these regimens compared with cefotaxime plus ampicillin is difficult to assess due to different definitions of infection used in the available studies and variability of study design (many single-center cohort studies) in different countries. One prospective nonrandomized study found no difference in the frequency of SSIs in orthotopic liver transplant recipients with cefazolin alone and amoxicillin-clavulanic acid alone, both given one hour before surgical incision, with a second dose given in cases of significant bleeding or surgery lasting over six hours, as antimicrobial prophylaxis [935]. The study did find a significantly higher rate of $A$. baumannii in the cefazolin group than the amoxicillin-clavulanic acid group. The routine use of vancomycin as antimicrobial prophylaxis is not recommended because of the risk of developing vancomycin-resistant organisms $[8,950]$, but vancomycin may be reserved for centers with an MRSA or MRSE cluster [8,950,957-959]. No randomized controlled studies have been conducted to compare the efficacy of other antimicrobial prophylactic regimens in the prevention of early postoperative infections. For patients known to be colonized with MRSA, VRE, or resistant gram-negative pathogens, it is reasonable to consider prophylaxis specifically targeted at these organisms. See the Common Principles section for further discussion.

Postoperative infections with Candida species after liver transplantation are common, particularly in the abdomen, and are frequently considered organ/space SSIs. For this reason, the use of antifungal prophylaxis in the perioperative period has become common. Efficacy has been demonstrated for fluconazole [964-984], lipid complex amphotericin B [985-987], and caspofungin [988]. Finally, one meta-analysis found a decreased risk of fungal infection and death associated with fungal infection, though not overall mortality, among patients given antifungal prophylaxis [989]. Universal antifungal prophylaxis is probably not necessary, since the risk of invasive candidiasis is low in uncomplicated cases. Instead, prophylaxis is generally reserved for patients with two or more of the following risk factors: need for reoperation, retransplantation, renal failure, choledochojejunostomy, and known colonization with Candida species [15]. Risk is also increased with prolonged initial procedure or transfusion of $>40$ units of cellular blood products, but this cannot be predicted before the procedure.

Selective bowel decontamination to eliminate aerobic gramnegative bacilli and yeast from the bowel before the transplantation procedure has been evaluated in several studies and a meta-analysis [936,943,949,955,956,967,968,980,990,991]. These studies used combinations of nonabsorbable antibacterials (aminoglycosides, polymyxin B or E), antifungals (nystatin, amphotericin B), and other antimicrobials (cefuroxime in suspension) administered orally and applied to the oropharyngeal cavity in combination with systemically administered antimicrobials. Results are conflicting, with no differences in patient outcomes (e.g., infection rates, mortality) or cost and concerns of increasing gram-positive infections with potential resistance in several studies $[939,955,956,980,991]$ and others with positive results $[936,949]$. Two randomized controlled studies found significantly fewer bacterial infections with early enteral nutrition plus lactobacillus and fibers [971,980]. Based on currently available data, the routine use of selective bowel decontamination or lactic acid bacteria and fibers in patients undergoing liver transplantation is not recommended.

Duration. No studies have assessed the optimal duration of antimicrobial prophylaxis in liver transplantation. Although antimicrobials have been administered in studies for five days [937,944,946,949,957-959] and seven days [964], the majority of recent studies have limited the duration of prophylaxis to $72 \mathrm{~h}[981], 48 \mathrm{~h}[936,943,945,952,955,956,960,961$, $967,970,979,980,991], 36$ h [981], 24 h [935,948,962,970], and a single dose [963], with no apparent differences in early infection rates. A prospective, nonrandomized, controlled study found no difference in bacterial infections within the first three months after liver transplantation in patients receiving cefotaxime and ampicillin as short-term antimicrobial prophylaxis for two to three days, compared with long-term prophylaxis for five to seven days [954]. Of note, five of the 11 patients in the long-term prophylaxis group had detectable C. difficile toxin B in the feces and developed enteritis. No patients in the short-term group had detectable C. difficile. Two recent review articles noted that antimicrobial prophylaxis duration should be less than three days $[896,950]$.

\section{Pediatric efficacy}

There are few data specifically concerning antimicrobial prophylaxis in liver transplantation in pediatric patients. The combination of cefotaxime plus ampicillin has been reportedly used in children undergoing living-related donor liver transplantation; the efficacy of this regimen appeared to be favorable [946]. A small, retrospective, single-center cohort study reported outcomes of children undergoing liver, heart, small bowel, or lung transplantation receiving piperacillin-tazobactam 120-150 mg/kg/day beginning before surgical incision and continuing for $48 \mathrm{~h}$ postoperatively and found favorable results, with a superficial SSI rate of $8 \%$ and no deep SSIs [992]. 


\section{Recommendations}

The recommended agents for patients undergoing liver transplantation are (1) piperacillin-tazobactam and (2) cefotaxime plus ampicillin (Table 2). (Strength of evidence for prophylaxis $=$ B.) For patients who are allergic to $\beta$-lactam antimicrobials, clindamycin or vancomycin given in combination with gentamicin, aztreonam, or a fluoroquinolone is a reasonable alternative. The duration of prophylaxis should be restricted to $24 \mathrm{~h}$ or less. For patients at high risk of Candida infection, fluconazole adjusted for renal function may be considered. (Strength of evidence for prophylaxis = B.)

\section{Pancreas and pancreas-kidney transplantation}

\section{Background}

Pancreas transplantation is an accepted therapeutic intervention for type 1 diabetes mellitus; it is the only therapy that consistently achieves euglycemia without dependence on exogenous insulin [993-997]. Simultaneous pancreas-kidney (SPK) transplantation is an accepted procedure for patients with type 1 diabetes and severe diabetic nephropathy. In 2007, UNOS reported that 469 pancreas transplantations and 862 SPK transplantations were performed in the United States, of which 60 and four patients, respectively, were under age 18 years [998]. Pancreas graft one-year survival rates ranged from $70.2 \%$ to $89 \%$, and the three-year rates ranged from $48 \%$ to $85.8 \%$ [998-1002]. Patient survival with pancreas transplantation has been reported between $75 \%$ and $97 \%$ at one year and between $54 \%$ and $92.5 \%$ at three years [998]. Allograft survival is higher in recipients of SPK transplantations, with allograft survival rates of $86.1-95.1 \%$ at one year and $54.2-92.5 \%$ at three years. Reported patient survival rates in SPK are $91.7-97.6 \%$ at one year and $84.4-94.1 \%$ at three years. During pancreas transplantation, surgical complications with portal-hepatic drainage significantly decreased the one-year and three-year survival rates to $48 \%$ and $44 \%$, respectively, in one cohort study [999].

Infectious complications are a major source of morbidity and mortality in patients undergoing pancreas or SPK transplantation; the frequency of SSI is $7 \%-50 \%$ with antimicrobial prophylaxis [993-997,1000-1009]. The majority of SSIs occurred within the first $30 \mathrm{~d}$ to three months after transplantation [1000-1002,1005,1008,1009]. Urinary tract infections are also a significant concern during the same time frame, with rates ranging from $10.6 \%$ to $49 \%$ in pancreas transplant recipients who received antimicrobial prophylaxis, and are much more common in recipients with bladder drainage compared with enteric drainage [1000-1008].

Pancreas and SPK transplantation patients may be at increased risk of SSIs and other infections because of the combined immunosuppressive effects of diabetes mellitus and the immunosuppressive drugs used to prevent graft rejection $[995,1000]$. Other factors associated with increased SSI rates include prolonged operating and ischemic times $(>4 \mathrm{~h}$ ), organ donor age of $>55$ years, and enteric rather than bladder drainage of pancreatic duct secretions [895,995,1000]. Prolonged organ preservation time $(>20 \mathrm{~h})$ was shown to increase the risk of complications, including duodenal leaks and decreased graft survival in cadaveric pancreas transplant recipients [1003]. Risk factors for UTI are reviewed in the kidney transplant section.

\section{Organisms}

A majority of superficial SSIs after pancreas or SPK transplantation are caused by Staphylococcus species (both coagulase-positive and coagulase-negative) and gram-negative bacilli (particularly E. coli and Klebsiella species) [993997,1000-1002,1004-1006,1009-1011]. Deep incisional SSIs also are frequently associated with gram-positive (Enterococcus species, Streptococcus species, and Peptostreptococcus species) and gram-negative organisms (Enterobacter species, Morganella species, and B. fragilis), as well as Candida species [993-997,10001002,1004-1006,1009-1011]. Although anaerobes are occasionally isolated, the necessity for specific treatment of anaerobes in SSIs after pancreas transplantation remains unclear.

\section{Efficacy}

Although no placebo-controlled studies have been conducted, several open-label, noncomparative, single-center studies have suggested that antimicrobial prophylaxis substantially decreases the rate of superficial and deep SSIs after pancreas or SPK transplantation. SSI rates were 7-33\% with various prophylactic regimens [995,1000-1002,1004,1005], compared with $7-50 \%$ for historical controls in the absence of prophylaxis $[1009,1010]$. The reason for the wide disparity in infection rates observed with prophylaxis is not readily apparent but may include variations in SSI definitions, variations in antimicrobial prophylaxis, immunosuppression protocols, and variations in surgical techniques [999-1002,1005,1007,1008].

Choice of agent. Because of the broad range of potential pathogens, several studies have used multidrug prophylactic regimens, including imipenem-cilastatin plus vancomycin [995]; tobramycin, vancomycin, and fluconazole [1010]; cefotaxime, metronidazole, and vancomycin [1012]; cefotaxime, vancomycin, and fluconazole [1008]; ampicillin and cefotaxime [1007]; and piperacillin-tazobactam and fluconazole [1006].

The HICPAC recommendations for SSI prevention include limiting the use of vancomycin unless there is an MRSA or MRSE cluster or as an alternative for $\beta$-lactam-allergic patients, though transplantation procedures were not specifically covered in the guidelines [8]. Limited data are available on the use of vancomycin as antimicrobial prophylaxis in kidney or pancreas transplantation, or both. A small, randomized, active-controlled, single-center study evaluated the impact of vancomycin-containing antimicrobial prophylaxis regimens in kidney and pancreas (alone or SPK) transplant recipients on the frequency of gram-positive infections [1004]. Renal transplantation patients received either vancomycin and ceftriaxone or cefazolin, and pancreas transplantation patients received either vancomycin and gentamicin or cefazolin and gentamicin. There was no statistically significant difference in the risk of developing gram-positive infections between antimicrobial prophylaxis regimens with and without vancomycin. The study was not powered to detect a difference in efficacy between the antimicrobial regimens. For patients known to be colonized with MRSA, VRE, or resistant gram-negative pathogens, it is reasonable to consider prophylaxis targeted specifically for these organisms. See the Common Principles section for further discussion.

An evaluation of the surgical complications of pancreas transplant recipients with portal-enteric drainage found an intraabdominal infection rate of $12 \%$ in the 65 patients 
undergoing SPK transplantation and no cases in those undergoing pancreas transplantation alone [999]. All patients received either cefazolin $1 \mathrm{~g}$ i.v. every eight hours for one to three days, or vancomycin if the patient had a $\beta$-lactam allergy.

One study evaluated SSI rates in SPK transplantation after single-agent, single-dose prophylaxis with cefazolin $1 \mathrm{~g}$ i.v. to donors and recipients, as well as cefazolin $1 \mathrm{~g} / \mathrm{L}$ bladder and intraabdominal irrigation in the recipient [1009]. Superficial SSIs developed in two patients (5\%), and deep SSIs associated with bladder anastomotic leaks or transplant pancreatitis occurred in four additional patients $(11 \%)$. This study reported similar SSI rates as with multidrug, multidose regimens.

Based on the regularity of isolation of Candida species from SSIs after pancreas transplantation and the frequent colonization of the duodenum with yeast, fluconazole is commonly added to prophylactic regimens. Although never studied in a randomized trial, a lower fungal infection rate was found in one large case series with the use of fluconazole $(6 \%)$ compared with no prophylaxis (10\%) [1013]. Although enteric drainage of the pancreas has been identified as a risk factor for postoperative fungal infections, many institutions use fluconazole for prophylaxis with bladder-drained organs as well. In settings with a high prevalence of non-albicans Candida species, a lipid-based formulation of amphotericin B has been recommended in infectious diseases guidelines from the American Society of Transplantation and the American Society of Transplant Surgeons [15].

Duration. Studies evaluating the use of antimicrobial prophylaxis regimens in pancreas and SPK transplantation, summarized above, ranged from a single preoperative dose of cefazolin to multidrug regimens of $2-5 \mathrm{~d}$ duration [995,1005,1009,1010,1012]. More recent studies reported monotherapy regimens with cefazolin or vancomycin [999], amoxicillin-clavulanic acid [1001,1002], and piperacillintazobactam [1000-1002] 1-7 d in duration, with the majority using the regimen $48-72 \mathrm{~h}$ after transplantation. The duration of fluconazole ranged from 7 to $28 \mathrm{~d}$ [1002].

\section{Recommendations}

The recommended regimen for patients undergoing pancreas or SPK transplantation is cefazolin (Table 2). (Strength of evidence for prophylaxis $=$ A.) For patients who are allergic to $\beta$-lactam antimicrobials, clindamycin or vancomycin given in combination with gentamicin, aztreonam, or a fluoroquinolone is a reasonable alternative. The duration of prophylaxis should be restricted to 24 hours or less. The use of aminoglycosides in combination with other nephrotoxic drugs may result in renal dysfunction and should be avoided unless alternatives are contraindicated. (Strength of evidence for prophylaxis $=\mathrm{C}$.) For patients at high risk of Candida infection, fluconazole adjusted for renal function may be considered.

\section{Kidney transplantation}

\section{Background}

In 2007, UNOS reported that 16,628 kidney transplantations were performed in the United States; of these, 796 patients were younger than 18 years [998]. The rate of postoperative infection after this procedure has been reported to range from $10 \%$ to $56 \%$, with the two most common in- fections being UTIs and SSIs [1004,1014-1024]. Graft loss due to infection occurs in up to $33 \%$ of cases [1017,1023]. One study of adult and pediatric kidney transplant recipients (both living-related and cadaveric donor sources) found patient survival rates at seven years after transplantation of $88.9 \%$ and $75.5 \%$, respectively, and graft survival of $75 \%$ and 55.5\%, respectively [1025]. No patients developed an SSI. Mortality associated with postoperative infections is substantial and ranges from approximately $5 \%$ to $30 \%$ $[1015,1017,1019,1022,1026,1027]$.

The frequency of SSIs in kidney transplant recipients has ranged from zero to $11 \%$ with antimicrobial prophylaxis [1023-1025,1028,1029] to $2 \%$ to $7.5 \%$ without systemic prophylaxis [1030,1031]. The majority of these infections were superficial in nature and were detected within 30 days after transplantation [1023,1028-1030]. Risk factors for SSI after kidney transplantation include contamination of organ perfusate [1027]; pretransplantation patient-specific factors, such as diabetes [1029,1030], chronic glomerulonephritis [1030], and obesity [1027,1030,1032]; procedure-related factors, such as ureteral leakage and hematoma formation [1027]; immunosuppressive therapy $[1024,1027,1029]$; and postoperative complications, such as acute graft rejection, reoperation, and delayed graft function [1030]. In one study, the frequency of SSI was $12 \%$ in patients receiving immunosuppression with azathioprine plus prednisone but only $1.7 \%$ in patients receiving cyclosporine plus prednisone [1033]. A significant difference in SSI rates was noted after kidney transplantation between immunosuppression regimens including mycophenolate mofetil (45 [3.9\%] of 1150 patients) versus sirolimus (11 [7.4\%] of 144 patients) [1029]. Sirolimus-containing immunosuppression was found to be an independent risk factor for SSIs. These recommendations refer to kidney transplant recipients; recommendations for living kidney donors can be found in the discussion of nephrectomy in the urologic section.

\section{Organisms}

Postoperative SSIs in kidney transplant recipients are caused by gram-positive organisms, particularly Staphylococcus species (including S. aureus and S. epidermidis) and Enterococcus species, gram-negative organisms, E. coli, Enterobacter species, Klebsiella species, P. aeruginosa, and yeast with Candida species [1004,1014-1021,1023,1024,1026,1028, 1030,1034]. One study site in Brazil reported a high level of antimicrobial resistance [1030]. Organisms recovered from infections included MRSA (77\%), methicillin-resistant coagulase-negative Staphylococcus (53.5\%), extended-spectrum $\beta$-lactamase-producing K. pneumoniae (80\%), and carbapenem-resistant $P$. aeruginosa (33.3\%). Another center in Brazil reported a significant difference in resistance to broadspectrum antimicrobials in pathogens isolated in UTIs from cadaveric kidney transplant recipients $(n=21,19.1 \%)$ compared with living-related donor kidney transplant recipients $(n=2,3.7 \%)(p=0.008)$ [1024]. One center in the United States reported $94 \%$ susceptibility to vancomycin of Enterococcus species within the first month after transplantation, while $E$. coli, cultured most commonly more than six months after transplantation, was $63 \%$ resistant to sulfamethoxazole-trimethoprim [1023]. This resistance may be related to the routine use of sulfamethoxazole-trimethoprim in prophylaxis of Pneumocystis jerovici pneumonia and UTI. 


\section{Efficacy}

A number of studies have clearly demonstrated that antimicrobial prophylaxis significantly decreases postoperative infection rates in patients undergoing kidney transplantation. These have included at least one randomized controlled trial [1014] and many prospective and retrospective studies comparing infection rates with prophylaxis and historical infection rates at specific transplantation centers [1015-1018,1021,10331035]. Based on the available literature, the routine use of systemic antimicrobial prophylaxis is justified in patients undergoing kidney transplantation.

Two studies that evaluated a triple-drug regimen consisting of an aminoglycoside, an antistaphylococcal penicillin, and ampicillin found infection rates of $<2 \%$, compared with $10-25 \%$ with no antimicrobial prophylaxis [1018,1019]. More specifically, infection rates in patients without antimicrobial prophylaxis (45 cadaveric and 44 living-related donors) were $10.1 \%$ in total $(8.9 \%$ and $11.4 \%$, respectively), compared with $1.5 \%$ in total (1.5\% and zero, respectively) with antimicrobial prophylaxis [1018]. Infection rates were as high as 33\% in living-related patients with no antimicrobial prophylaxis and zero to $1 \%$ in both cadaveric and living-related transplant recipients with antimicrobial prophylaxis [1021]. Piperacillin plus cefuroxime was also shown to be efficacious; infection rates were $3.7 \%$, compared with $19 \%$ in cadaveric transplant recipients not receiving prophylaxis [1018]. Several studies have shown that single-agent prophylaxis with an antistaphylococcal penicillin $[1029,1034]$, a first-generation cephalosporin [1016,1017,1023,1024,1029], a second-generation cephalosporin $[1028,1035,1036]$, or a third-generation cephalosporin (e.g., cefoperazone, cefotaxime, ceftriaxone) [1024,1029, $1033,1037]$ can reduce postoperative infection rates to zero to $8.4 \%$. All studies included cadaveric transplant recipients, whereas living-related transplant recipients were also included in select studies $[1017,1024,1028,1036]$. Where compared directly, infection rates between cadaveric and living-related transplant recipients receiving antimicrobial prophylaxis were not statistically different [1024].

Choice of agent. The available data do not indicate a significant difference between single-drug and multidrug antimicrobial regimens [1014,1018,1021]. In addition, there appears to be no significant differences between single-agent regimens employing antistaphylococcal penicillins and first-, second-, or third-generation cephalosporins [1016,1017,1033$1035,1037]$. Studies have directly compared antimicrobial regimens in a prospective, controlled fashion. Single-agent prophylaxis with both cefazolin and ceftriaxone has been reported to result in SSI rates of zero [1016,1024,1037].

A survey of 101 kidney transplant centers in 39 countries reported that $65 \%$ of the centers used single antimicrobial prophylaxis regimens, $20.8 \%$ used two-drug regimens, and $3 \%$ used three drugs; no prophylaxis was used in $11 \%$ of centers [1036]. Cephalosporins were used in 68 centers (55 alone, 7 in combination with penicillin, and 6 with other antimicrobials). Penicillins were used by 28 centers (13 alone, 7 with cephalosporin, and 8 with other antimicrobials). Other antimicrobials (specifics were not reported) were used in two centers as the single agent.

As noted above, HICPAC recommendations for SSI prevention include limiting the use of vancomycin to situations in which there is an MRSA or MRSE cluster or as an alternative for $\beta$-lactam-allergic patients [8]. Transplantation procedures were not specifically covered in the guidelines.

Duration. Studies have used various prophylactic regimens, ranging from a single-drug cephalosporin regimen, administered as a single preoperative dose or for up to $24 \mathrm{~h}$ postoperatively, to multidrug regimens of two to five days' duration [981,1004,1014-1018,1021,1023,1024,1028,1029,1033, $1036,1038]$. Cefazolin for $24 \mathrm{~h}$ was equivalent to seven days of surgical prophylaxis in living-related kidney transplant donors. [1039] There appear to be no significant differences in SSI rates between single-dose, 24-h, and multidose regimens; therefore, the duration of antimicrobial should be restricted to $24 \mathrm{~h}$.

\section{Pediatric efficacy}

Although pediatric patients were included in studies demonstrating the efficacy of antimicrobial prophylaxis, there are few data specific to pediatric patients. One cohort of 96 pediatric patients who underwent 104 renal transplants $(63 \%$ cadaveric and $37 \%$ living-related donors) ranged in age from six months to 18 years (mean age, $8.2 \pm 5.5$ years) [1040]. Antimicrobial prophylaxis included one dose of cefotaxime $30-\mathrm{mg} /$ $\mathrm{kg}$ i.v. bolus at the start of the procedure and cefotaxime $90 \mathrm{mg} /$ $\mathrm{kg}$ /day in three divided doses during the intensive care unit stay, which averaged one to two days. No SSIs were reported.

\section{Recommendations}

The recommended agent for patients undergoing kidney transplantation is cefazolin (Table 2). (Strength of evidence for prophylaxis $=$ A.) For patients who are allergic to $\beta$-lactam antimicrobials, clindamycin or vancomycin given in combination with gentamicin, aztreonam, or a fluoroquinolone is a reasonable alternative. The duration of prophylaxis should be restricted to $24 \mathrm{~h}$ or less. The use of aminoglycosides in combination with other nephrotoxic drugs may result in renal dysfunction and should be avoided unless alternatives are contraindicated. (Strength of evidence for prophylaxis $=$ C.) For patients at high risk of Candida infection, fluconazole adjusted for renal function may be considered.

\section{Plastic surgery and breast procedures}

\section{Background}

Plastic surgery encompasses a broad range of procedures focused on reconstructive, dermatological, and cosmetic procedures [1041]. The primary goal of these procedures is to restore function to the affected area, with a secondary goal of improving appearance. The scope of procedures ranges from simple primary surgical site closure, skin grafts, and skin flaps to composite tissue transplantations. Composite tissue transplantation for tissue reconstruction of the knee joint, larynx, uterus, abdominal wall, hand, face, and penis has been performed in a small number of patients [1042,1043].

Most dermatological, breast (reduction and reconstructive), clean head and neck, and facial procedures have an associated SSI rate of <5\% [1044-1053]. Oral procedures, such as wedge excision of lip or ear, flaps on the nose [1046,1054], and head and neck flaps, have SSI rates of approximately 5-10\% [1053,1055-1060]. In addition to general risk factors as described in the Common Principles section, factors that increase the risk of postoperative infectious complications 
for plastic surgery procedures include implants [1061], skin irradiation before the procedure, and procedures below the waist [1062,1063].

\section{Organisms}

The most common organisms in SSIs after plastic surgery procedures are S. aureus $[1045,1049,1050,1053,1054,1056$, 1063-1068], other staphylococci, and streptococci [1045, $1054,1064,1066,1067]$. Procedures involving macerated, moist environments (e.g., under a panus or axilla of an obese individual), below the waist, or in patients with diabetes are associated with a higher rate of infection with gram-negative organisms such as P. aeruginosa, [1068] Serratia marcescens, or Enterobacteriaceae, including E. coli [1065,1068], Klebsiella species [1068], and P. mirabilis [1065].

\section{Efficacy}

The efficacy of antimicrobial prophylaxis in select plastic surgery procedures has been investigated in several clinical trials and cohort studies. Most placebo-controlled and retrospective studies for many clean plastic surgery procedures have found that antimicrobial prophylaxis does not significantly decrease the risk of infection. These studies have evaluated head and neck procedures (facial bone fracture, tumor excision and reconstruction, radical neck dissection, rhinoplasty) [1049], flexor tendon injury repairs [1051], augmentation mammoplasty using periareolar submuscular technique [1052], carpal tunnel [1069], and breast procedures (reduction mammoplasty, lumpectomy, mastectomy, axillary node dissection) $[1056,1058,1070,1071]$.

However, a Cochrane review of seven randomized, placebo-controlled trials of 1984 patients undergoing breast cancer procedures (axillary lymph node dissection and primary nonreconstructive surgery) evaluated the effectiveness of preoperative or perioperative antimicrobial prophylaxis $(n=995)$ compared with placebo or no treatment $(n=989)$ in reducing the rate of postoperative infections [1072]. Pooled study results revealed a significant difference in SSI rates with antimicrobial prophylaxis (80 [8\%] of 995), compared with $10.5 \%$ (104 of 989$)$ for no antimicrobial prophylaxis (relative risk, 0.72; 95\% CI, 0.53-0.97). Review authors concluded that antimicrobial prophylaxis is warranted to decrease the risk of SSIs in nonreconstructive breast cancer procedures.

Guidelines also support no antimicrobial prophylaxis in patients undergoing clean facial or nasal procedures without an implant [7]. For patients undergoing facial or nasal procedures with an implant, antimicrobial prophylaxis should be considered [7].

A randomized, double-blind, controlled trial of 207 patients evaluated the use of three antimicrobial prophylaxis regimens in patients undergoing abdominoplasty procedures [1066]. The reported SSI rates were 13\% for patients receiving no antimicrobial prophylaxis, $4.3 \%$ for those receiving preoperative antimicrobials only, and $8.7 \%$ for those receiving one preoperative dose and three days of postoperative antimicrobials. There was a significantly lower infection rate in the group receiving preoperative antimicrobials only compared with the placebo group $(\mathrm{p}<0.05)$. The infection rate was slightly but not significantly higher in patients who received postoperative antimicrobials.
Choice of agent. There is no consensus on the appropriate antimicrobial agent to use for prophylaxis in plastic surgery procedures $[1055,1073]$. Agents with good gram-positive coverage and, depending on the site of surgery, activity against common gram-negative organisms are recommended for patients undergoing clean plastic surgery procedures with risk factors (listed in the Common Principles section and the background discussion of this section) or clean-contaminated procedures. Cefazolin or ampicillin-sulbactam is sufficient in most cases, with clindamycin and vancomycin as alternatives for patients with $\beta$-lactam allergy. There are no studies assessing the impact of MRSA on patients undergoing plastic surgery procedures or regarding the need to alter prophylaxis regimens in patients without known colonization with MRSA. When vancomycin or clindamycin is used and if a gram-negative organism is highly suspected, practitioners should consider adding cefazolin if the patient is not $\beta$-lactam allergic; if the patient is $\beta$-lactam allergic, the addition of aztreonam, gentamicin, or single-dose fluoroquinolone should be considered. If the surgical site involves the ear, an antipseudomonal fluoroquinolone may be considered to cover Pseudomonas species [1045].

Although oral agents such as cephalexin, amoxicillin, clindamycin, and azithromycin have been recommended in reviews of antimicrobial prophylaxis in clean dermatological surgery, there is no evidence that supports their use $[13,1045,1046,1054]$.

Duration. Antimicrobial prophylaxis should be limited to the shortest duration possible to prevent SSIs (even if a drain or a catheter is left in place or an implant is inserted), limit adverse events, and prevent antimicrobial resistance $[8,512,1047,1048,1054,1056]$. Multiple studies have found no significant differences in SSI rates after breast surgery with single-dose preoperative cephalosporin compared with extended-duration regimens that last from one to five days postoperatively [1048,1054,1056].

A randomized, single-blind, controlled trial of 74 patients undergoing surgical ablation of head and neck malignancies with immediate free-flap reconstruction found no significant differences in SSI rate between clindamycin $900 \mathrm{mg}$ i.v. every eight hours for three doses compared with 15 doses [1057]. Both groups were given clindamycin $900 \mathrm{mg}$ i.v. immediately preoperatively, in addition to the postoperative regimens.

In a controlled study, 200 patients undergoing septorhinoplasty were randomized to a single preoperative dose of amoxicillin-clavulanate $2.2 \mathrm{~g}$ i.v. administered $30 \mathrm{~min}$ before surgical incision only $(n=100)$ or in combination with postoperative oral amoxicillin-clavulanic acid $1000 \mathrm{mg}$ twice daily for seven days [533]. There was no significant difference in infection rates between the group receiving only a preoperative dose (zero) and the combination group (3\%). There was a higher rate of adverse events (nausea, diarrhea, skin rash, and pruritus) among the combination group compared with the group receiving only a preoperative dose $(p=0.03)$. The study authors recommended the use of a single preoperative i.v. dose of amoxicillin-clavulanate for endonasal septorhinoplasty.

\section{Pediatric efficacy}

Limited data on antimicrobial prophylaxis are available for pediatric patients undergoing plastic surgery procedures. 
There is no consensus among surgeons regarding the use of antimicrobial prophylaxis in the repair of cleft lip and palate [1074]. The occurrence of postoperative infections after these procedures is $1.3 \%$ [1075]. No controlled trials have evaluated the use of antimicrobial prophylaxis in these procedures.

\section{Recommendations}

Antimicrobial prophylaxis is not recommended for most clean procedures in patients without additional postoperative infection risk factors as listed in the Common Principles section of these guidelines and the background discussion of this section. Although no studies have demonstrated antimicrobial efficacy in these procedures, expert opinion recommends that patients with risk factors undergoing clean plastic procedures receive antimicrobial prophylaxis. The recommendation for clean-contaminated procedures, breast cancer procedures, and clean procedures with other risk factors is a single dose of cefazolin or ampicillin-sulbactam (Table 2). (Strength of evidence for prophylaxis $=\mathrm{C}$.) Alternative agents for patients with $\beta$-lactam allergy include clindamycin and vancomycin. If there are surveillance data showing that gramnegative organisms cause SSIs for the procedure, the practitioner may consider combining clindamycin or vancomycin with another agent (cefazolin if the patient is not $\beta$-lactamallergic; aztreonam, gentamicin, or single-dose fluoroquinolone if the patient is $\beta$-lactam-allergic). Postoperative duration of antimicrobial prophylaxis should be limited to less than $24 \mathrm{~h}$, regardless of the presence of indwelling catheters or drains.

\section{Acknowledgments}

The following individuals are acknowledged for their significant contributions to this manuscript: Sandra I. Berríos-Torres, M.D.; Rachel Bongiorno-Karcher, Pharm.D.; Colleen M. Culley, Pharm.D., BCPS; Susan R. Dombrowski, M.S., B.S.Pharm.; and Susan J. Skledar, B.S.Pharm., M.P.H., FASHP.

Financial support provided by Emory University, Johns Hopkins University, Northwestern University, Rush University, University of Colorado, University of Michigan, University of Oklahoma, University of Nebraska, University of Virginia, University of Washington, and West Virginia University.

Dr. Bratzler is a consultant for Telligen; Dr. Dellinger has received honoraria for participation on advisory boards and consultation for Merck, Baxter, Ortho-McNeil, Targanta, Schering-Plough, WebEx, Astellas, Durata, Pfizer, Applied Medical, Rib-X, 3M, the American Hospital Association, Premier Inc., Oklahoma Foundation for Medical Quality, and the Hospital Association of New York State; Dr. Perl serves on the advisory boards of Hospira and Pfizer and has received a grant from Merck; Dr. Auwaerter serves on the advisory panel of Genentech; Dr. Fish serves on the advisory board and speakers' bureau of Merck; and Dr. Sawyer serves as a consultant for Pfizer, Merck, Wyeth, 3M, and Ethicon and has received an R01 grant from the National Institute of General Medical Sciences and a T32 grant from the National Institute of Allergy and Infectious Diseases. Drs. Bolon, Napolitano, Olsen, Steinberg, Slain, and Weinstein have declared no potential conflicts of interest.

\section{References}

1. American Society of Health-System Pharmacists. ASHP therapeutic guidelines on antimicrobial prophylaxis in surgery. Am J Health-Syst Pharm 1999; 56:1839-1888.

2. Dellinger EP, Gross PA, Barrett TL et al. Quality standard for antimicrobial prophylaxis in surgical procedures. $\underline{\text { Clin }}$ Infect Dis 1994; 18:422-427.

3. Page CP, Bohnen JM, Fletcher JR et al. Antimicrobial prophylaxis for surgical wounds: guidelines for clinical care. Arch Surg 1993; 128:79-88.

4. Dotson LR, Witmer DR. Development of ASHP therapeutic guidelines. Am J Health-Syst Pharm 1995; 52:254255.

5. Anderson DJ, Kaye KS, Classen D et al. Strategies to prevent surgical site infections in acute care hospitals. Infect Control Hosp Epidemiol 2008; 29(suppl 1):S51-S61.

6. Antimicrobial prophylaxis for surgery. Treat Guidel Med Lett 2009; 7:47-52.

7. Scottish Intercollegiate Guidelines Network. Antibiotic prophylaxis in surgery. www.sign.ac.uk/pdf/sign104.pdf (accessed 2009 Jul 30).

8. Mangram AJ, Horan TC, Pearson ML et al. Guideline for prevention of surgical site infection. Infect Control Hosp Epidemiol 1999; 20:250-278.

9. American College of Obstetricians and Gynecologists. Antibiotic prophylaxis for gynecologic procedures. ACOG practice bulletin no. 104. Obstet Gynecol 2009; 113:11801189.

10. AAP Comittee on Fetus and Newborn, ACOG Committee on Obstetric Practice, eds. Guidelines for perinatal care. 6th ed. Elk Grove Village, IL: American Academy of Pediatrics and American College of Obstetricians and Gynecologists; 2008.

11. Wilson W, Taubert KA, Gewitz M et al. Prevention of infective endocarditis: guidelines from the American Heart Association: a guideline from the American Heart Association Rheumatic Fever, Endocarditis, and Kawasaki Disease Committee, Council on Cardiovascular Disease in the Young, and the Council on Clinical Cardiology, Council on Cardiovascular Surgery and Anesthesia, and the Quality of Care and Outcomes Research Interdisciplinary Working Group. Circulation 2007; 116:1736-1754.

12. Freiman JA, Chalmers TC, Smith $\mathrm{H}$ et al. The importance of beta, the type II error and sample size in the design and interpretation of the randomized control trial. N Engl J Med 1978; 299:690-694.

13. Edmiston CE, Krepel C, Kelly H et al. Perioperative antibiotic prophylaxis in the gastric bypass patient: do we achieve therapeutic levels? Surgery 2004; 136:738-747.

14. Claforan (cefotaxime sodium) for injection package insert. Bridgewater, NJ: Sanofi Aventis; 2009 Jul.

15. Pappas PG, Silveira FP. Candida in solid organ transplant recipients. Am J Transplant 2009; 9(suppl 4):S173-179.

16. Zelenitsky SA, Silverman RE, Duckworth $\mathrm{H}$ et al. A prospective, randomized, double-blind study of single high dose versus multiple standard dose gentamicin both in combination with metronidazole for colorectal surgical prophylaxis. J Hosp Infect 2000; 46:135-140.

17. Zelenitsky SA, Ariano RE, Harding GK et al. Antibiotic pharmacodynamics in surgical prophylaxis: an association between intraoperative antibiotic concentrations and efficacy. Antimicrob Agents Chemother 2002; 46:3026-3030.

18. Rybak MJ, Lomaestro BM, Rotschafer JC et al. Vancomycin therapeutic guidelines: a summary of consensus recommendations from the Infectious Diseases Society of 
America, the American Society of Health-System Pharmacists, and the Society of Infectious Diseases Pharmacists. Clin Infect Dis 2009; 49:325-327.

19. Alphonso N, Anagnostopoulos PV, Scarpace $S$ et al. Perioperative antibiotic prophylaxis in paediatric cardiac surgery. Cardiol Young 2007; 17:12-25.

20. Maher $\mathrm{KO}$, VanDerElzen $\mathrm{K}$, Bove EL et al. A retrospective review of three antibiotic prophylaxis regimens for pediatric cardiac surgical patients. Ann Thorac Surg 2002; 74:1195-1200.

21. Kato Y, Shime N, Hashimoto S et al. Effects of controlled perioperative antimicrobial prophylaxis on infectious outcomes in pediatric cardiac surgery. Crit Care Med 2007; 35:1763-1768.

22. Haessler D, Reverdy ME, Neidecker J et al. Antibiotic prophylaxis with cefazolin and gentamicin in cardiac surgery for children less than ten kilograms. I Cardiothorac Vasc Anesth 2003; 17:221-225.

23. Vargas MR, Danton MH, Javaid SM et al. Pharmacokinetics of intravenous flucloxacillin and amoxicillin in neonatal and infant cardiopulmonary bypass surgery. Eur J Cardiothorac Surg 2004; 25:256-260.

24. Milstone AM, Budd A, Shepard JW et al. Role of decolonization in a comprehensive strategy to reduce methicillin-resistant Staphylococcus aureus infections in the neonatal intensive care unit: an observational cohort study. Infect Control Hosp Epidemiol 2010; 31: 558-560.

25. Chua AN, Goldstein SL, Bell D et al. Topical mupirocin/ sodium hypochlorite reduces peritonitis and exit-site infection rates in children. Clin J Am Soc Nephrol 2009; 4:1939-1943.

26. Paglialonga F, Esposito S, Edefonti A et al. Catheterrelated infections in children treated with hemodialysis. Pediatr Nephrol 2004; 19:1324-1333.

27. Shiojima T, Ohki Y, Nako Y et al. Immediate control of a methicillin-resistant Staphylococcus aureus outbreak in a neonatal intensive care unit. J Infect Chemother 2003; 9:243-247.

28. Romance L, Nicolle L, Ross J et al. An outbreak of methicillin-resistant Staphylococcus aureus in a pediatric hospital-how it got away and how we caught it. Can J Infect Control 1991; 6:11-13.

29. Hayakawa T, Hayashidera T, Katsura S et al. Nasal mupirocin treatment of pharynx-colonized methicillin resistant Staphylococcus aureus: preliminary study with 10 carrier infants. Pediatr Int 2000; 42:67-70.

30. Liu CC, Hor LI, Wu YH et al. Investigation and elimination of epidemic methicillin-resistant Staphylococcus aureus in a neonatal intensive care unit. Min Kuo Hsiao Erh Ko I Hsueh Hui Tsa Chih. (Chinese Medical Journal) 1993; 34:285-293.

31. Nateghian A, Taylor G, Robinson JL. Risk factors for surgical site infections following open-heart surgery in a Canadian pediatric population. Am J Infect Control 2004; 32:397-401.

32. Bratzler DW, Houck PM, Richards C et al. Use of antimicrobial prophylaxis for major surgery: baseline results from the National Surgical Infection Prevention Project. Arch Surg 2005; 140:174-182.

33. National Healthcare Safety Network. Patient safety component manual: surgical site infection (SSI) event. www.cdc.gov/nhsn/PDFs/pscManual/9pscSSIcurrent .pdf (accessed 2011 Apr 5).
34. Horan TC, Gayness RP, Martone WJ et al. CDC definitions of nosocomial surgical site infections, 1992: a modification of CDC definitions of surgical wound infections. Infect Control Hosp Epidemiol 1992; 13:606-608.

35. National Healthcare Safety Network. Patient safety component manual: key terms. www.cdc.gov/nhsn/PDFs/pscManual/ 16pscKeyTerms_current.pdf (accessed 2012 Oct 23).

36. Horan TC, Andrus M, Dudeck MA. CDC/NHSN surveillance definition of health care-associated infection and criteria for specific types of infections in the acute care setting. Am J Infect Control 2008; 36:309-332.

37. Ehrenkranz NJ, Pfaff SJ. Mediastinitis complicating cardiac operations: evidence of postoperative causation. $\underline{\mathrm{Rev}}$ Infect Dis 1991; 13:803-814.

38. Bowater RJ, Stirling SA, Lilford RJ. Is antibiotic prophylaxis in surgery a generally effective intervention? Testing a generic hypothesis over a set of meta-analyses. Ann Surg 2009; 249:551-556.

39. Englesbe MJ, Dimick JB, Sonnenday CJ et al. The Michigan Surgical Quality Collaborative: will a statewide quality improvement initiative pay for itself? Ann Surg 2007; 246:1100-1103.

40. Flum DR, Fisher N, Thompson J et al. Washington state's approach to variability in surgical processes/outcomes: Surgical Clinical Outcomes Assessment Program (SCOAP). Surgery 2005; 138:821-828.

41. Bratzler DW, Houck PM, for the Surgical Infection Prevention Guidelines Writers Workgroup. Antimicrobial prophylaxis for surgery: an advisory statement from the national surgical infection prevention project. $\underline{\text { Clin Infect }}$ Dis 2004; 38:1706-1715.

42. Fry DE. Surgical site infections and the Surgical Care Improvement Project (SCIP): evolution of national quality measures. Surg Infect 2008; 9:579-584.

43. Watson DS. National patient safety goals and implementation. AORN J 2009; 90:123-127.

44. Myles JL, Shamanski F, Witte D. The Physicians Quality Reporting Initiative: measure, development, implementation and current procedural terminology coding. Adv Anat Pathol 2010; 17:49-52.

45. Callcut RA, Breslin TM. Shaping the future of surgery: the role of private regulation in determining quality standards. Ann Surg 2006; 243:304-312.

46. Bratzler DW. The Surgical Infection Prevention and Surgical Care Improvement Projects: promises and pitfalls. Am Surg 2006; 72:1010-1016.

47. DePalma RG. Surgical quality programs in the Veterans Health Administration. Am Surg 2006; 72:999-1004.

48. Gómez MI, Acosta-Gnass SI, Mosqueda-Barboza L et al. Reduction in surgical antibiotic prophylaxis expenditure and the rate of surgical site infection by means of a protocol that controls the use of prophylaxis. Infect Control Hosp Epidemiol 2006; 27:1358-1365.

49. Bond CA, Raehl CL. Clinical and economic outcomes of pharmacist-managed antimicrobial prophylaxis in surgical patients. Am J Health-Syst Pharm 2007; 64:19351942.

50. Hermsen ED, Smith Shull S, Puumala SE et al. Improvement in prescribing habits and economic outcomes associated with the introduction of a standardized approach for surgical antimicrobial prophylaxis. Infect Control Hosp Epidemiol 2008; 29:457-461.

51. Alerany C, Campany D, Monterde J et al. Impact of local guidelines and an integrated dispensing system on 
antibiotic prophylaxis quality in a surgical centre. J Hosp Infect 2005; 60:111-117.

52. Allerberger F, Gareis R, Jindrák V et al. Antibiotic stewardship implementation in the EU: the way forward. Expert Rev Anti Infect Ther 2009; 7:1175-1183.

53. Voit SB, Todd JK, Nelson B et al. Electronic surveillance system for monitoring surgical antimicrobial prophylaxis. Pediatrics 2005; 116:1317-1322.

54. Kritchevsky SB, Braun BI, Bush AJ et al. The effect of a quality improvement collaborative to improve antimicrobial prophylaxis in surgical patients: a randomized trial. Ann Intern Med 2008; 149:472-480.

55. Potenza B, Deligencia M, Estigoy B et al. Lessons learned from the institution of the Surgical Care Improvement Project at a teaching medical center. Am J Surg 2009; 198:881-888.

56. Parker BM, Henderson JM, Vitagliano $S$ et al. Six sigma methodology can be used to improve adherence for antibiotic prophylaxis in patients undergoing noncardiac surgery. Anesth Analg 2007; 104:140-146.

57. Rosenberg AD, Wambold D, Kraemer L et al. Ensuring appropriate timing of antimicrobial prophylaxis. I Bone Joint Surg Am 2008; 90:226-232.

58. Manniën J, Van Kasteren ME, Nagelkerke NJ et al. Effect of optimized antibiotic prophylaxis on the incidence of surgical site infection. Infect Control Hosp Epidemiol 2006; 27:13401346.

59. Gorbach SL, Condon RE, Conte JE Jr et al. Evaluation of new anti-infective drugs for surgical prophylaxis. Clin Infect Dis 1992; 15(suppl 1):S313-S338.

60. Källman J, Friberg Ö. Antibiotic prophylaxis in cardiac surgery-general principles. APMIS 2007; 115:1012-1015.

61. Hidron AI, Edwards JR, Patel J et al., for the National Healthcare Safety Network Team and participating National Healthcare Safety Network facilities. Antimicrobialresistant pathogens associated with healthcare-associated infections: annual summary of data reported to the National Healthcare Safety Network at the Centers for Disease Control and Prevention, 2006-2007. Infect Control Hosp Epidemiol 2008; 29:996-1011.

62. Cefotetan package insert. Shaumburg, IL: Abraxis Pharmaceutical Products; 2007 Jul.

63. Cefazolin package insert. Shaumburg, IL: Abraxis Pharmaceutical Products; 2006 Jul.

64. Cefoxitin package insert. Shaumburg, IL: APP Pharmaceuticals, LLC; 2008 Feb.

65. Sterile vancomycin package insert. Shaumburg, IL: APP Pharmaceuticals, LLC; 2008 Apr.

66. Cefuroxime package insert. Shaumburg, IL: APP Pharmaceuticals; 2008 Nov.

67. Invanz (ertapenem injection) package insert. Whitehouse Station, NJ: Merck; 2010 Mar.

68. Gaynes R, Edwards JR. National Nosocomial Infections Surveillance System: overview of nosocomial infections caused by gram-negative bacilli. Clin Infect Dis 2005; 41:848-854

69. Weigelt JA, Lipsky BA, Tabak UP et al. Surgical site infections: causative pathogens and associated outcomes. Am J Infect Control 2010; 38:112-120.

70. Centers for Disease Control and Prevention. Recommendations for preventing the spread of vancomycin resistance. Recommendations of the Hospital Infection Control Practices Advisory Committee (HICPAC). www .cdc.gov/mmwr/PDF/RR/RR4412.PDF (accessed 2012 Dec 9).
71. Gould FK, Brindle R, Chadwick PR et al. Guidelines (2008) for the prophylaxis and treatment of methicillinresistant Staphylococcus aureus (MRSA) infections in the United Kingdom. J Antimicrob Chemother 2009; 63: 849-861.

72. Bolon MK, Morlote M, Weber SG et al. Glycopeptides are no more effective than $\beta$-lactam agents for prevention of surgical site infection after cardiac surgery: a metaanalysis. Clin Infect Dis 2004; 38:1357-1363.

73. Finkelstein R, Rabino $G$, Mashiah $T$ et al. Vancomycin versus cefazolin prophylaxis for cardiac surgery in the setting of a high prevalence of methicillin-resistant staphylococcal infections. J Thorac Cardiovasc Surg 2002; 123:326332.

74. Bull AL, Worth LJ, Richards MJ. Impact of vancomycin surgical prophylaxis on the development of methicillinsensitive Staphylococcus aureus surgical site infections: report from Australian surveillance data (VICNISS). Ann Surg Epub ahead of print. 2012 Jul 20 (DOI 10.1097/ SLA.0b013e31825fa398).

75. Gorwitz RJ, Kruszon-Moran D, McAllister SK et al. Changes in the prevalence of nasal colonization with Staphylococcus aureus in the United States, 2001-2004. J Infect Dis 2008; 197:1226-1234.

76. Milstone AM, Carroll KC, Ross T et al. Community-associated methicillin-resistant Staphylococcus aureus strains in pediatric intensive care unit. Emerg Infect Dis 2010; 16:647-655.

77. Lo WT, Wang CC, Lin WJ et al. Changes in the nasal colonization with methicillin-resistant Staphylococcus aureus in children: 2004-2009. PLoS One 2010; 5:e15791.

78. Roberts NJ, Douglas RG. Gentamicin use and Pseudomonas and Serratia resistance: effect of a surgical prophylaxis regimen. Antimicrob Agents Chemother 1978; 13:214-220.

79. Kriesel D, Savel TG, Silver AL et al. Surgical antibiotic prophylaxis and Clostridium difficile toxin positivity. Arch Surg 1995; 130:989-993.

80. Privitera G, Scarpellini P, Ortisi G et al. Prospective study of Clostridium difficile intestinal colonization and disease following single-dose antibiotic prophylaxis surgery. Antimicrob Agents Chemother 1991; 35:208-210.

81. Jobe BA, Grasley A, Deveney KE et al. Clostridium difficile colitis: an increasing hospital-acquired illness. Am J Surg 1995; 169:480-483.

82. Morris JG, Shay DK, Hebden JN. Enterococci resistant to multiple antimicrobial agents, including vancomycin: establishment of endemicity in a university medical center. Ann Intern Med 1995; 123:250-259.

83. Sastry V, Brennan PJ, Levy MM. Vancomycin-resistant enterococci: an emerging problem in immunosuppressed transplant recipients. Transplant Proc 1995; 27:954-955.

84. Rhinehart E, Smith NE, Wennersten C. Rapid dissemination of beta-lactamase producing aminoglycoside resistant Enterococcus faecium among patients and staff on an infanttoddler surgical ward. N Engl J Med 1990; 323:1814-1818.

85. Cohen SH, Gerding DN, Johnson S et al. Clinical practice guidelines for Clostridium difficile infection in adults: 2010 update by the Society for Healthcare Epidemiology of America (SHEA) and the Infectious Diseases Society of America (IDSA). Infect Control Hosp Epidemiol 2010; 31:431-455.

86. Salkind AR, Cuddy PG, Foxworth JW. Is this patient allergic to penicillin? An evidence-based analysis of the likelihood of penicillin allergy. JAMA 2001; 285:24982505. 
87. Frumin J, Gallagher JC. Allergic cross-sensitivity between penicillin, carbapenem, and monobactam antibiotics: what are the chances? Ann Pharmacother 2009; 43:304315.

88. Cunha BA. Antibiotic selection in the penicillin-allergic patient. Med Clin North Am 2006; 90:1257-1264.

89. Pichichero ME. Use of selected cephalosporins in penicillinallergic patients: a paradigm shift. Diagn Microbiol Infect Dis 2007; 57:13s-18s.

90. Galandiuk S, Polk HC Jr, Jagelman DG et al. Re-emphasis of priorities in surgical antibiotic prophylaxis. Surg Gynecol Obstet 1989; 169:218-222.

91. DiPiro JT, Vallner JJ, Bowden TA et al. Intraoperative serum and tissue activity of cefazolin and cefoxitin. Arch Surg 1985; 120:829-832.

92. Classen DC, Evans RS, Pestotnik SL et al. The timing of prophylactic administration of antibiotics and the risk of surgical-wound infection. N Engl J Med 1992; 326:281286.

93. Garey KW, Dao T, Chen $\mathrm{H}$ et al. Timing of vancomycin prophylaxis for cardiac surgery patients and the risk of surgical site infections. J Antimicrob Chemother 2006; 58:645650.

94. Steinberg JP, Braun BI, Hellinger WC et al. Timing of antimicrobial prophylaxis and the risk of surgical site infection: results from the Trial to Reduce Antimicrobial Prophylaxis Errors. Ann Surg 2009; 250:10-16.

95. Van Kasteren ME, Mannien J, Ott A et al. Antibiotic prophylaxis and the risk of surgical site infections following total hip arthroplasty: timely administration is the most important factor. Clin Infect Dis 2007; 44:921-927.

96. Soriano A, Bori G, Garcia-Ramiro $S$ et al. Timing of antibiotic prophylaxis for primary total knee arthroplasty performed during ischemia. Clin Infect Dis 2008; 46: 1009-1014.

97. Weber WP, Marti WR, Zwahlen $M$ et al. The timing of surgical antimicrobial prophylaxis. Ann Surg 2008; 247: 918-926.

98. Dellinger EP. What is the ideal time for administration of antimicrobial prophylaxis for a surgical procedure? Ann Surg 2008; 247:927-928.

99. Goldman DA, Hopkins CC, Karchmer AW et al. Cephalothin prophylaxis in cardiac valve surgery. A prospective, double-blind comparison of two-day and six-day regimens. J Thorac Cardiovasc Surg 1977; 73:470-479.

100. Platt R, Munoz A, Stella J et al. Antibiotic prophylaxis for cardiovascular surgery. Efficacy with coronary artery bypass. Ann Intern Med 1984; 101:770-774.

101. Forse RA, Karam B, MacLean LD et al. Antibiotic prophylaxis for surgery in morbidly obese patients. Surgery 1989; 106:750-756.

102. Falagas ME, Karageorgopoulos DE. Adjustment of dosing of antimicrobial agents for bodyweight in adults. Lancet 2010; 375:248-251.

103. Pai MP, Bearden DT. Antimicrobial dosing considerations in obese adult patients. Insights from the Society of Infectious Diseases Pharmacists. Pharmacotherapy 2007; 27:1081-1091.

104. Johnson PN, Miller JL, Boucher EA, for the Pediatric Pharmacy Advisory Group Advocacy Committee. Medication dosing in overweight and obese children. www .ppag.org/obesedose (accessed 2010 Nov 22).

105. Koopman E, Nix DE, Erstad BL et al. End-of-procedure cefazolin concentrations after administration for preven- tion of surgical-site infection. Am J Health-Syst Pharm 2007; 64:1927-1934.

106. Bauer LA, Edwards WA, Dellinger EP et al. Influence of weight on aminoglycoside pharmacokinetics in normal weight and morbidly obese patients. Eur J Clin Pharmacol 1983; 24:643-647.

107. Bailey TC, Little JR, Littenberg B et al. A meta-analysis of extended-interval dosing versus multiple daily dosing of aminoglycosides. Clin Infect Dis 1997; 24:786-795.

108. Barza M, Ioannidis JP, Cappelleri JC et al. Single or multiple daily doses of aminoglycosides: a meta-analysis. $\underline{\mathrm{BMJ}}$ 1996; 312:338-345.

109. Blaser J, Konig C. Once-daily dosing of aminoglycosides. Eur J Clin Microbiol Infect Dis 1995; 14:1029-1038.

110. Murry KR, McKinnon PS, Mitrzyk B et al. Pharmacodynamic characterization of nephrotoxicity associated with once-daily aminoglycoside. Pharmacotherapy 1999; 19:1252-1260.

111. Nicolau DP, Freeman CD, Belliveau PP et al. Experience with a once-daily aminoglycoside program administered to 2,184 adult patients. Antimicrob Agents Chemother 1995; 39:650-655.

112. Rao SC, Ahmed M, Hagan R. One dose per day compared to multiple doses per day of gentamicin for treatment of suspected or proven sepsis in neonates. Cochrane Database Syst Rev 2006; 1:CD005091.

113. Zhanel GG, Ariano RE. Once daily aminoglycoside dosing: maintained efficacy with reduced nephrotoxicity? Ren Fail 1992; 14:1-9.

114. Anaya DA, Dellinger EP. The obese surgical patient: a susceptible host for infection. Surg Infect 2006; 7:473-480.

115. Waisbren E, Rosen H, Bader AM et al. Percent body fat and prediction of surgical site infection. I Am Coll Surg 2010; 210:381-389.

116. Engelman R, Shahian D, Shemin R et al. The Society of Thoracic Surgeons practice guideline series: antibiotic prophylaxis in cardiac surgery, part II: antibiotic choice. Ann Thorac Surg 2007; 83:1569-1576.

117. Zanetti G, Giardina R, Platt R. Intraoperative redosing of cefazolin and risk for surgical site infection in cardiac surgery. Emerg Infect Dis 2001; 7:828-831.

118. Scher KS. Studies on the duration of antibiotic administration for surgical prophylaxis. Am Surg 1997; 63:59-62.

119. Markantonis SL, Kostopanagiotou G, Panidis D et al. Effects of blood loss and fluid volume replacement on serum and tissue gentamicin concentrations during colorectal surgery. Clin Ther 2004; 26:271-281.

120. Morita S, Nishisho I, Nomura T et al. The significance of the intraoperative repeated dosing of antimicrobials for preventing surgical wound infection in colorectal surgery. Surg Today 2005; 35:732-738.

121. Swoboda SM, Merz C, Kostuik J et al. Does intraoperative blood loss affect antibiotic serum and tissue concentrations? Arch Surg 1996; 131:1165-1672.

122. DiPiro JT, Cheung RP, Bowden TA et al. Single-dose systemic antibiotic prophylaxis of surgical wound infections. Am J Surg 1986; 152:552-559.

123. Fonseca SN, Kunzle SR, Junqueira MJ et al. Implementing 1-dose antibiotic prophylaxis for prevention of surgical site infection. Arch Surg 2006; 141:1109-1113.

124. McDonald M, Grabsch E, Marshall C et al. Single-versus multiple-dose antimicrobial prophylaxis for major surgery: a systematic review. Aust N Z J Surg 1998; 68: 388-396. 
125. Kreter B, Woods M. Antibiotic prophylaxis for cardiothoracic operations. Meta-analysis of thirty years of clinical trials. J Thorac Cardiovasc Surg 1992; 104:590-599.

126. Austin TW, Coles JC, Burnett R et al. Aortocoronary bypass procedures and sternotomy infections: a study of antistaphylococcal prophylaxis. Can J Surg 1980; 23:483485.

127. Galbraith U, Schilling J, Von Segesser LK et al. Antibiotic prophylaxis in cardiovascular surgery: a prospective, randomized, comparative trial of one-day cefazolin vs single-dose cefuroxime. Drugs Exp Clin Res 1993; 19:229234.

128. Kriaras I, Michalopoulos A, Michalis A et al. Antibiotic prophylaxis in cardiac surgery. J Cardiovasc Surg 1997; 38:605-610.

129. Kriaras I, Michalopoulos A, Turina M et al. Evolution of antimicrobial prophylaxis in cardiovascular surgery. Eur J Cardiothorac Surg 2000; 18:440-446.

130. Harbarth S, Samore MH, Lichtenberg D et al. Prolonged antibiotic prophylaxis after cardiovascular surgery and its effect on surgical site infections and antimicrobial resistance. Circulation 2000; 101:2916-2921.

131. Edwards FH, Engelman R, Houck P et al. The Society of Thoracic Surgeons practice guideline series: antibiotic prophylaxis in cardiac surgery, part I: duration. Ann Thorac Surg 2006; 81:397-404.

132. Lee KR, Ring JC, Leggiadro RJ. Prophylactic antibiotic use in pediatric cardiovascular surgery: a surgery of current practice. Pediatr Infect Dis J 1995; 14:267-269.

133. American Academy of Orthopaedic Surgeons. Information statement: recommendations for the use of intravenous antibiotic prophylaxis in primary total joint arthroplasty. www.aaos.org/about/papers/advistmt/1027 .asp (accessed 2008 May 13).

134. McCarthy PJ, Patil S, Conrad SA et al. International and specialty trends in the use of prophylactic antibiotics to prevent infectious complications after insertion of external ventricular drainage devices. Neurocrit Care 2010; 12: 220-224.

135. Hares MM, Hegarty MA, Warlow J et al. A controlled trial to compare systemic and intra-incisional cefuroxime prophylaxis in high risk gastric surgery. Br J Surg 1981; 68: 276-280.

136. Moesgaard F, Lykkegaard Nielsen M. Failure of topically applied antibiotics, added to systemic prophylaxis, to reduce perineal wound infection in abdominoperineal excision of the rectum. Acta Chir Scand 1988; 154:589-592.

137. Pitt HA, Postier RG, Gadacz TR et al. The role of topical antibiotics in "high-risk" biliary surgery. Surgery 1982; 91:518-524.

138. Pitt HA, Postier RG, Mcgowan WA et al. Prophylactic antibiotics in vascular surgery. Topical, systemic, or both. Ann Surg 1980; 192:356-364.

139. Schersten H. Modified prophylaxis for preventing deep sternal wound infection after cardiac surgery. APMIS 2007; 115:1023-1026.

140. Friberg Ö, Svedjeholm R, Söderquist B et al. Local gentamicin reduces sternal wound infections after cardiac surgery: a randomized controlled trial. Ann Thorac Surg 2005; 79:153-162.

141. Eklund AM, Valtonen M, Werkkala KA. Prophylaxis of sternal wound infections with gentamicin-collagen implant: randomized controlled study in cardiac surgery. I Hosp Infect 2005; 59:108-112.
142. Vander Salm TJ, Okike ON, Pasque MK et al. Reduction of sternal infection by application of topical vancomycin. J Thorac Cardiovasc Surg 1989; 98:618-622.

143. Bennett-Guerrero E, Ferguson TB Jr, Lin M et al. Effect of an implantable gentamicin-collagen sponge on sternal wound infections following cardiac surgery: a randomized trial. JAMA 2010; 304:755-762.

144. Bennett-Guerrero E, Pappas TN, Koltun WA et al. Gentamicin-collagen sponge for infection prophylaxis in colorectal surgery. N Engl J Med 2010; 363:1038-1049.

145. McHugh SM, Collins CJ, Corrigan MA et al. The role of topical antibiotics used as prophylaxis in surgical site infection prevention. J Antimicrob Chemother 2011; 66:693701.

146. Goodman J, Schaffner W, Collins H et al. Infection after cardiovascular surgery. N Engl J Med 1968; 278:117123.

147. Perl TM. Prevention of Staphylococcus aureus infections among surgical patients: beyond traditional perioperative prophylaxis. Surgery 2003; 134:S10-S17.

148. Kluytmans JA, Mouton JW, Ijzerman EP et al. Nasal carriage of Staphylococcus aureus as a major risk factor for wound infections after cardiac surgery. I Infect Dis 1995; 171:216-219.

149. Kluytmans JA, Mouton JW, Vanden-Bergh MF et al. Reduction of surgical-site infections in cardiothoracic surgery by elimination of nasal carriage of Staphylococcus aureus. Infect Control Hosp Epidemiol 1996; 17:780-785.

150. Kalmeijer MD, Coertjens H, Van Nieuwland-Bollen PM et al. Surgical site infections in orthopedic surgery: the effect of mupirocin nasal ointment in a double-blind, randomized, placebo-controlled study. Clin Infect Dis 2002; 35:353-358.

151. Hacek DM, Robb WJ, Paule SM et al. Staphylococcus aureus nasal decolonization in joint replacement surgery reduces infection. Clin Orthop Relat Res 2008; 466:1349-1355.

152. White A, Smith J. Nasal reservoir as the source of extranasal staphylococci. Antimicrob Agents Chemother 1963; 161:679-683.

153. Lauderdale TL, Wang JT, Lee WS et al. Carriage rates of methicillin-resistant Staphylococcus aureus (MRSA) depend on anatomic location, the number of sites cultured, culture methods, and the distribution of clonotypes. Eur J Clin Microbiol Infect Dis 2010; 29:1553-1559.

154. Jain R, Kralovic SM, Evans ME. Veterans Affairs initiative to prevent Staphylococcus aureus infections. N Engl J Med 2011; 364:1419-1430.

155. Harbarth S, Fankhauser C, Schrenzel J et al. Univeral screening for methicillin-resistant Staphylococcus aureus at hospital admission and nosocomial infection in surgical patients. IAMA 2008; 299:1149-1157.

156. Bactroban (mupirocin calcium ointment, $2 \%$ ) nasal package insert. Research Triangle Park, NC: GlaxoSmithKline; 2009 Apr.

157. Kallen AJ, Wilson CT, Larson RJ. Perioperative intranasal mupirocin for the prevention of surgical-site infections: systematic review of the literature and meta-analysis. Infect Control Hosp Epidemiol 2005; 26:916-922.

158. Van Rijen M, Bonten M, Wenzel R et al. Mupirocin ointment for preventing Staphylococcus aureus infections in nasal carriers. Cochrane Database Syst Rev 2008; 4:CD006216.

159. Hebert C, Robicsek A. Decolonization therapy in infection control. Curr Opin Infect Dis 2010; 23:340-345. 
160. Perl TM, Cullen JJ, Wenzel RP et al. Intranasal mupirocin to prevent postoperative Staphylococcus aureus infections. N Engl J Med 2002; 346:1871-1877.

161. Konvalinka A, Errett L, Fong IW. Impact of treating Staphylococcus aureus nasal carriers on wound infections in cardiac surgery. J Hosp Infect 2006; 64:162-168.

162. Bode LG, Kluytmans JA, Wertheim HF et al. Preventing surgical-site infections in nasal carriers of Staphylococcus aureus. N Engl J Med 2010; 362:9-17.

163. Lee AS, Macedo-Vinas M, Francois $\mathrm{P}$ et al. Impact of combined low-level mupirocin and genotypic chlorhexidine resistance on persistent methicillin-resistant Staphylococcus aureus carriage after decolonization therapy: a case-control study. Clin Infect Dis 2011; 52:1422-1430.

164. Muto CA, Jernigan JA, Ostrowsky BE et al. SHEA guideline for preventing nosocomial transmission of multidrug-resistant strains of Staphylococcus aureus and Enterococcus. Infect Control Hosp Epidemiol 2003; 24:362386.

165. Edwards JR, Peterson KD, Mu Y et al. National Healthcare Safety Network (NHSN) report: data summary for 2006 through 2008. Am J Infect Control 2009; 37:783-805.

166. Kutsal A, Ibrisim E, Catav Z et al. Mediastinitis after open heart surgery. Analysis of risk factors and management. J Cardiovasc Surg 1991; 32:38-41.

167. Abboud CS, Way SB, Baltar VT. Risk factors for mediastinitis after cardiac surgery. Ann Thorac Surg 2004; 77:676683.

168. Crabtree TD, Codd JE, Fraser VJ et al. Multivariate analysis of risk factors for deep and superficial sternal infection after coronary artery bypass grafting at a tertiary care medical center. Semin Thorac Cardiovasc Surg 2004; 16: 53-61.

169. Kohli M, Yuan L, Escobar M et al. A risk index for sternal surgical wound infection after cardiovascular surgery. Infect Control Hosp Epidemiol 2003; 24:17-25.

170. Lepelletier D, Perron S, Bizouarn P et al. Surgical-site infection after cardiac surgery: incidence, microbiology and risk factors. Infect Control Hosp Epidemiol 2005; 26: 466-472.

171. Lu JC, Grayson AD, Jha P et al. Risk factors for sternal wound infection and mid-term survival following coronary artery bypass surgery. Eur J Cardiothorac Surg 2003; 23:943-949.

172. Tang GH, Maganti M, Weisel RD et al. Prevention and management of deep sternal wound infection. Semin Thorac Cardiovasc Surg 2004; 16:62-69.

173. Jakob HG, Borneff-Lipp M, Bach A et al. The endogenous pathway is a major route for deep sternal wound infection. Eur J Cardiothorac Surg 2000; 17:154-160.

174. Rahmanian PB, Adams DH, Castillo JG et al. Impact of body mass index on early outcome and late survival in patients undergoing coronary artery bypass grafting or valve surgery or both. Am J Cardiol 2007; 100:17021708.

175. Vuorisalo S, Haukipuro K, Pokela R et al. Risk features for surgical-site infections in coronary artery bypass surgery. Infect Control Hosp Epidemiol 1998; 19:240-247.

176. Segers P, De Jong AP, Kloek JJ et al. Risk control of surgical site infection after cardiothoracic surgery. J Hosp Infect 2006; 62:437-445.

177. Zerr KJ, Furnary AP, Grunkemeier GL et al. Glucose control lowers the risk of wound infection in diabetics after open heart operations. Ann Thorac Surg 1997; 63:356-361.
178. Furnary AP, Zerr KJ, Grunkemeier GL et al. Continuous intravenous insulin infusion reduces the incidence of deep sternal wound infection in diabetic patients after cardiac surgical procedures. Ann Thorac Surg 1999; 67:352-362.

179. Dellinger EP. Preventing surgical-site infections: the importance of timing and glucose control. Infect Control Hosp Epidemiol 2001; 22:604-606.

180. Latham R, Lancaster AD, Covington JF et al. The association of diabetes and glucose control with surgical-site infections among cardiothoracic surgery patients. Infect Control Hosp Epidemiol 2001; 22:607-612.

181. Furnary AP, Gao G, Grunkemeier GL et al. Continuous insulin infusion reduces mortality in patients with diabetes undergoing coronary artery bypass grafting. I Thorac Cardiovasc Surg 2003; 125:1007-1021.

182. Gandhi GY, Nuttall GA, Abel MD et al. Intraoperative hyperglycemia and perioperative outcomes in cardiac surgery patients. Mayo Clin Proc 2005; 80:862-866.

183. Dohmen PM. Influence of skin flora and preventive measures on surgical site infection during cardiac surgery. Surg Infect 2006; 7:S13-S17.

184. Kittle C, Reed W. Antibiotics and extracorporeal circulation. I Thorac Cardiovasc Surg 1961; 41:34-48.

185. Slonim R, Litwak R, Gadboys $\mathrm{H}$ et al. Antibiotic prophylaxis of infection complicating open-heart operations. Antimicrob Agents Chemother 1963; 3:731-735.

186. Garey KW, Amrutkar P, Dao-Tran TK et al. Economic benefit of appropriate timing of vancomycin prophylaxis in patients undergoing cardiovascular surgery. Pharmacotherapy 2008; 28:699-706.

187. Garey KW, Lai D, Dao-Tran TK et al. Interrupted time series analysis of vancomycin compared to cefuroxime for surgical prophylaxis in patients undergoing cardiac surgery. Antimicrob Agents Chemother 2008;52: 446-451.

188. Cimochowski GE, Harostock MD, Brown R et al. Intranasal mupirocin reduces sternal wound infection after open heart surgery in diabetics and nondiabetics. Ann Thorac Surg 2001; 71:1572-1579.

189. Zangrillo A, Landoni G, Fumagalli L et al. Methicillinresistant Staphylococcus species in a cardiac surgical intensive care unit: a 5-year experience. I Cardiothorac Vasc Anesth 2006; 20:31-37.

190. Fekety F, Cluff L, Sabiston D et al. A study of antibiotic prophylaxis in cardiac surgery. I Thorac Cardiovasc Surg 1969; 57:757-763.

191. Conte J, Cohen S, Roe B et al. Antibiotic prophylaxis and cardiac surgery: a prospective double-blind comparison of single-dose versus multi-dose regimens. Ann Intern Med 1972; 76:943-949.

192. Firor W. Infection following open-heart surgery, with special reference to the role of prophylactic antibiotics. I Thorac Cardiovasc Surg 1967; 53:371-378.

193. Slama T, Sklar S, Misinski J et al. Randomized comparison of cefamandole, cefazolin and cefuroxime prophylaxis in open-heart surgery. Antimicrob Agents Chemother 1986; 29:744-747.

194. Kaiser A, Petracek M, Lea J et al. Efficacy of cefazolin, cefamandole, and gentamicin as prophylactic agents in cardiac surgery. Ann Surg 1987; 206:791-797.

195. Conklin C, Gray R, Neilson D et al. Determinants of wound infection incidence after isolated coronary artery bypass surgery in patients randomized to receive 
prophylactic cefuroxime or cefazolin. Ann Thorac Surg 1988; 46:172-177.

196. Wellens F, Pirlet M, Larbuisson R et al. Prophylaxis in cardiac surgery: a controlled, randomized comparison between cefazolin and cefuroxime. Eur J Cardiothorac Surg 1995; 9:325-329.

197. Townsend TR, Reitz BA, Bilker WB et al. Clinical trial of cefamandole, cefazolin, and cefuroxime for antibiotic prophylaxis in cardiac operations. I Thorac Cardiovasc Surg 1993; 106:664-670.

198. Curtis JJ, Boley TM, Walls JT et al. Randomized, prospective comparison of first- and second-generation cephalosporins as infection prophylaxis for cardiac surgery. Am J Surg 1993; 166:734-737.

199. Doebbeling BN, Pfaller MA, Kuhns KR et al. Cardiovascular surgery prophylaxis: a randomized, controlled comparison of cefazolin and cefuroxime. I Thorac Cardiovasc Surg 1990; 99:981-989.

200. Vuorisalo S, Pokela R, Syrjälä H. Comparison of vancomycin and cefuroxime for infection prophylaxis in coronary artery bypass surgery. Infect Control Hosp Epidemiol 1998; 19:234-239.

201. Baddour LM, Bettmann MA, Bolger AF et al. Nonvalvular cardiovascular device-related infections. Circulation 2003; 108:2015-2031.

202. Chamber CE, Eisenhauer MD, McNicol LB et al. Infection control guidelines for the cardiac catheterization laboratory: society guidelines revisited. Catheter Cardiovasc Interv 2006; 67:78-86.

203. Van Rijen M, Bonten M, Wenzel R et al. Mupirocin ointment for preventing Staphylococcus aureus infections in nasal carriers. Cochrane Database Syst Rev 2008; 4:CD006216.

204. Ortega GM, Martí-Bonmatí E, Guevara SJ et al. Alteration of vancomycin pharmacokinetics during cardiopulmonary bypass in patients undergoing cardiac surgery. Am J Health-Syst Pharm 2003; 60:260-265.

205. Nascimento JW, Carmona MJ, Strabelli TM et al. Systemic availability of prophylactic cefuroxime in patients submitted to coronary artery bypass grafting with cardiopulmonary bypass. J Hosp Infect 2005; 59:299-303.

206. Vuorisalo S, Pokela R, Syrjälä H. Is single-dose antibiotic prophylaxis sufficient for coronary artery bypass surgery? An analysis of peri- and postoperative serum cefuroxime and vancomycin levels. J Hosp Infect 1997; 37:237-247.

207. Fellinger EK, Leavitt BJ, Hebert JC. Serum levels of prophylactic cefazolin during cardiopulmonary bypass surgery. Ann Thorac Surg 2002; 74:1187-1190.

208. Caffarelli AD, Holden JP, Baron EJ et al. Plasma cefazolin levels during cardiovascular surgery: effects of cardiopulmonary bypass and profound hypothermic circulatory arrest. I Thorac Cardiovasc Surg 2006; 131:13381343.

209. Hutschala D, Skhirtladze $K$, Kinstner $C$ et al. In vivo microdialysis to measure antibiotic penetration into soft tissue during cardiac surgery. Ann Thorac Surg 2007; 84:16051610.

210. Waltrip T, Lewis R, Young V et al. A pilot study to determine the feasibility of continuous cefazolin infusion. Surg Infect 2002; 3:5-9.

211. Nascimento JW, Carmona MJ, Strabelli TM et al. Perioperative cefuroxime pharmacokinetics in cardiac surgery. Clinics 2007; 62:257-260.

212. Lewis DR, Longman TJ, Wisheart JD et al. The pharmacokinetics of a single dose of gentamicin $(4 \mathrm{mg} / \mathrm{kg})$ as prophylaxis in cardiac surgery requiring cardiopulmonary bypass. Cardiovasc Surg 1999; 7:398-401.

213. Kitzes-Cohen R, Farin D, Piva G et al. Pharmacokinetics of vancomycin administered as prophylaxis before cardiac surgery. Ther Drug Monit 2000; 22:661-667.

214. Austin T, Coles J, McKenzie P et al. Cephalothin prophylaxis and valve replacement. Ann Thorac Surg 1977; 23:333-336.

215. Sisto T, Laurikka J, Tarkka MR. Ceftriaxone vs cefuroxime for infection prophylaxis in coronary bypass surgery. Scand J Thorac Cardiovasc Surg 1994; 28:143-148.

216. Nooyen SM, Overbeek BP, Brutel De La Riviere A et al. Prospective randomised comparison of single-dose versus multiple-dose cefuroxime for prophylaxis in coronary artery bypass grafting. Eur J Clin Microbiol Infect Dis 1994; 13:1033-1037.

217. Tamayo E, Gualis J, Flórez S et al. Comparative study of single-dose and 24-hour multiple-dose antibiotic prophylaxis for cardiac surgery. J Thorac Cardiovasc Surg 2008; 136:1522-1527.

218. Mertz D, Johnstone J, Loeb M. Does duration of perioperative antibiotic prophylaxis matter in cardiac surgery? A systematic review and meta-analysis. Ann Surg 2011; 254:48-54.

219. Sandoe JA, Kumar B, Stoddart B et al. Effect of extended perioperative antibiotic prophylaxis on intravascular catheter colonization and infection in cardiothoracic surgery patients. J Antimicrob Chemother 2003; 52:877-879.

220. Niederhäuser U, Vogt M, Vogt P et al. Cardiac surgery in a high-risk group of patients: is prolonged postoperative antibiotic prophylaxis effective? J Thorac Cardiovasc Surg 1997; 114:162-168.

221. Huddleston CB. Mediastinal wound infections following pediatric cardiac surgery. Semin Thorac Cardiovasc Surg 2004; 16:108-112.

222. McEvoy GK, Snow EK, Miller J et al., eds. AHFS drug information. Bethesda, MD: American Society of HealthSystem Pharmacists; 2011.

223. Johns Hopkins Hospital, Arcara K, Tschudy M, Lee CK, eds. Harriet Lane handbook, 19th edition. Philadelphia: Elsevier, Inc.; 2012.

224. Lance LL, Lacey CF, Goldman MP et al., eds. Quick-look drug book, 18th edition. Baltimore, MD: Lippincott Williams \& Wilkins; 2011.

225. Lexi-Comp Online. Hudson, OH: Lexi-Comp, Inc.; 2011.

226. Smith KM, Riche DM, Henyan NN, eds. Handbook of clinical drug data. 11th ed. New York: McGrawHill; 2010.

227. Da Costa A, Kirkorian G, Cucherat M et al. Antibiotic prophylaxis for permanent pacemaker implantation: a meta-analysis. Circulation 1998; 97:1796-1801.

228. Nishimura RA, Carabello BA, Faxon DP et al. ACC/AHA 2008 guideline update on valvular heart disease: focused update on infective endocarditis: a report of the American College of Cardiology/American Heart Association Task Force on Practice Guidelines. Circulation 2008; 118:887-896.

229. Baddour LM, Epstein AE, Erickson CC et al. American Heart Association Rheumatic Fever, Endocarditis, and Kawasaki Disease Committee; Council on Cardiovascular Disease in Young; Council on Cardiovascular Surgery and Anesthesia; Council on Cardiovascular Nursing; Council on Clinical Cardiology; Interdisciplinary Council on Quality of Care; American Heart Association. Update on cardiovascular implantable electronic device infections 
and their management: a scientific statement from the American Heart Association. Circulation 2010; 121:458477.

230. Klug D, Balde M, Pavin D et al. Risk factors related to infections of implanted pacemakers and cardioverter-defibrillators: results of a large prospective study. Circulation 2007; 116:1349-1355.

231. De Oliveira JC, Martinelli M, Nishioka SA et al. Efficacy of antibiotic prophylaxis before the implantation of pacemakers and cardioverter-defibrillators: results of a large, prospective, randomized, double-blinded, placebocontrolled trial. Circ Arrhythm Electrophysiol 2009; 2: 29-34.

232. Sohail MR, Uslan DZ, Khan AH et al. Risk factor analysis of permanent pacemaker infection. Clin Infect Dis 2007; 45:166-173.

233. Bertaglia E, Zerbo F, Zardo S et al. Antibiotic prophylaxis with a single dose of cefazolin during pacemaker implantation: incidence of long-term infective complications. PACE 2006; 29:29-33.

234. Holman WL, Pae WE, Teutenberg JJ et al. INTERMACS: interval analysis of registry data. J Am Coll Cardiol 2009; 208:755-761.

235. Walker PC, DePrestel DD, Miles NA et al. Surgical infection prophylaxis for left ventricular assist device implantation. J Card Surg 2011; 26:440-443.

236. Califano S, Pagani FD, Malani PN. Left ventricular assist device-associated infections. Infect Dis Clin North Am 2012; 26:77-87.

237. Radu DM, Jauréguy F, Seguin A et al. Postoperative pneumonia after major pulmonary resections: an unsolved problem in thoracic surgery. Ann Thorac Surg 2007; 84:1669-1674.

238. Cardo D, Horan T, Andres M et al. National Nosocomial Infections Surveillance (NNIS) system report: data summary from January 1992 through June 2004. Am J Infect Control 2004; 32:470-485.

239. Aznar R, Mateu M, Miró JM et al. Antibiotic prophylaxis in non-cardiac thoracic surgery: cefazolin versus placebo. Eur J Cardiothorac Surg 1991; 5:515-518.

240. Rovera F, Imperatori A, Militello P et al. Infections in 346 consecutive video-assisted thoracoscopic procedures. Surg Infect 2003; 4:45-51.

241. Turna A, Kutlu CA, Ozalp T et al. Antibiotic prophylaxis in elective thoracic surgery: cefuroxime versus cefepime. Thorac Cardiov Surg 2003; 51:84-88.

242. Boldt J, Piper S, Uphus D et al. Preoperative microbiologic screening and antibiotic prophylaxis in pulmonary resection operations. Ann Thorac Surg 1999; 68:208-211.

243. Schussler O, Dermine H, Alifano $M$ et al. Should we change antibiotic prophylaxis for lung surgery? Postoperative pneumonia is the critical issue. Ann Thorac Surg 2008; 86:1727-1734.

244. Shiono S, Yoshida J, Nishimura M et al. Risk factors of postoperative respiratory infections in lung cancer surgery. J Thorac Oncol 2007; 2:34-38.

245. Imperatori A, Rotolo N, Gatti M et al. Peri-operative complications of video-assisted thoracoscopic surgery (VATS). Int J Surg 2008; 6:S78-S81.

246. Solaini L, Prusciano F, Bagioni P et al. Video-assisted thoracic surgery (VATS) of the lung: analysis of intraoperative and postoperative complications over 15 years and review of the literature. Surg Endosc 2008; 22:298-310.
247. Imperatori A, Rovera F, Rotolo N et al. Prospective study of infection risk factors in 988 lung resections. Surg Infect 2006; 7:S57-S60.

248. Chamberlain RS, Sakpal SV. A comprehensive review of single-incision laparoscopic surgery (SILS) and natural orifice transluminal endoscopic surgery (NOTES) techniques for chelecystectomy. I Gastrointest Surg 2009; 13: 1733-1740.

249. Cruse PJ, Foord R. The epidemiology of wound infection: a ten-year prospective study of 62,939 wounds. Surg Clin North Am 1980; 60:27-40.

250. Petrosillo N, Drapeau CM, Nicastri E et al. Surgical site infections in Italian hospitals: a prospective multicenter study. BMC Infect Dis 2008; 8:34.

251. Watanabe A, Kohnoe S, Shimabukuro R et al. Risk factors associated with surgical site infection in upper and lower gastrointestinal surgery. Surg Today 2008; 38:404-412.

252. Yasuda K, Shiraishi N, Adachi Y et al. Risk factors for complications following resection of large gastric cancer. Br J Surg 2001; 88:873-877.

253. Mohri Y, Tonouchi H, Kobayashi M et al. Randomized clinical trial of single-versus multiple-dose antimicrobial prophylaxis in gastric cancer surgery. Br J Surg 2007; 94: 683-688.

254. Yeo CJ, Cameron JL, Sohn TA et al. Pancreaticoduodenectomy with or without extended retroperitoneal lymphadenectomy for periampullary adenocarcinoma: comparison of morbidity and mortality and short-term outcome. Ann Surg 1999; 229:613-624.

255. Jafri NS, Mahid SS, Minor KS et al. Meta-analysis: antibiotic prophylaxis to prevent peristomal infection following percutaneous endoscopic gastrostomy. Aliment Pharmacol Ther 2007; 25:647-656.

256. Sharma VK, Howden CW. Meta-analysis of randomized, controlled trials of antibiotic prophylaxis before percutaneous endoscopic gastrostomy. Am J Gastroenterol 2000; 95:3133-3136.

257. Gorbach SL, Plaut AG, Nahas L et al. Studies of intestinal microflora: II. Microorganisms of the small intestine and their relations to oral fecal flora. Gastroenterology 1967; 53:856-867.

258. Gorbach SL. Intestinal microflora. Gastroenterology 1971; 60:1110-1129.

259. Ruddell WS, Axon AT, Findlay JM et al. Effect of cimetidine on the gastric bacterial flora. Lancet 1980; 1:672-674.

260. Driks MR, Craven DE, Bartolome RC et al. Nosocomial pneumonia in intubated patients given sucralfate as compared with antacids or histamine type 2 blockers. $\underline{\mathrm{N}}$ Engl J Med 1987; 317:1376-1382.

261. Long J, Desantis S, State D et al. The effect of antisecretagogues on gastric microflora. Arch Surg 1983; 118:1413-1415.

262. Sjostedt S, Levin P, Malmborg AS et al. Septic complications in relation to factors influencing the gastric microflora in patients undergoing gastric surgery. I Hosp Infect 1989; 12:191-197.

263. Feretis CB, Contou CT, Papoutsis GG et al. The effect of preoperative treatment with cimetidine on postoperative wound sepsis. Am Surg 1984; 50:594-598.

264. Antimicrobial prophylaxis in surgery. Treat Guidel Med Lett 2006; 4:83-88.

265. LoCiero J, Nichols RL. Sepsis after gastroduodenal operations; relationship to gastric acid, motility, and endogenous microflora. South Med J 1980; 73:878-880. 
266. Pessaux P, Msika S, Atalla D et al. Risk factors for postoperative infectious complications in noncolorectal abdominal surgery: a multivariate analysis based on a prospective multicenter study of 4718 patients. Arch Surg 2003; 138:314-324.

267. Imai E, Ueda M, Kanao K et al. Surgical site infection surveillance after open gastrectomy and risk factors for surgical site infection. I Infect Chemother 2005; 11:141145.

268. Christou NV, Jarand J, Sylvestre JL et al. Analysis of the incidence and risk factors for wound infections in open bariatric surgery. Obes Surg 2004; 14:16-22.

269. McArdle CS, Morran CG, Pettit L et al. Value of oral antibiotic prophylaxis in colorectal surgery. Br J Surg 1995; 82:1046-1048.

270. Nichols RL, Webb WR, Jones JW et al. Efficacy of antibiotic prophylaxis in high risk gastroduodenal operations. Am J Surg 1982; 143:94-98.

271. Stone HH. Gastric surgery. South Med J 1977; 70:S35-S37.

272. Morris DL, Yound D, Burdon DW et al. Prospective randomized trial of single-dose cefuroxime against mezlocillin in elective gastric surgery. J Hosp Infect 1984; 5:200-204.

273. Lewis RT, Allan CM, Goodall RG et al. Discriminate use of antibiotic prophylaxis in gastroduodenal surgery. Am J Surg 1979; 138:640-643.

274. Lewis RT, Allan CM, Goodall RG et al. Cefamandole in gastroduodenal surgery: a controlled prospective, randomized, double-blind study. Can J Surg 1982; 25:561563.

275. Mitchell NJ, Evans DS, Pollock D. Pre-operative, singledose cefuroxime antimicrobial prophylaxis with and without metronidazole in elective gastrointestinal surgery. J Antimicrob Chemother 1980; 6:393-399.

276. Uchiyama K, Takifuji K, Tani M et al. Prevention of postoperative infections by administration of antimicrobial agents immediately before surgery for patients with gastrointestinal cancers. Hepatogastroenterology 2007; 54: 1487-1493.

277. Pories WJ, Van Rij AM, Burlingham BT et al. Prophylactic cefazolin in gastric bypass surgery. Surgery 1981; 90: 426-432.

278. Polk H, Lopez-Meyer J. Postoperative wound infection: a prospective study of determinant factors and prevention. Surgery 1969; 66:97-103.

279. Saadeddin A, Freshwater DA, Fisher NC et al. Antibiotic prophylaxis for percutaneous endoscopic gastrostomy for non-malignant conditions: a double-blind prospective randomized controlled trial. Aliment Pharmacol Ther 2005; 22:565-570.

280. Ahmad I, Mouncher A, Abdoolah A et al. Antibiotic prophylaxis for percutaneous endoscopic gastrostomy-a prospective, randomized, double-blind trial. Aliment Pharmacol Ther 2003; 18:209-215.

281. Dormann AJ, Wigginghaus B, Risius H et al. Antibiotic prophylaxis in percutaneous endoscopic gastrostomy (PEG) - results from a prospective randomized multicenter trial. Z Gastroenterol 2000; 38:229-234.

282. Preclik G, Grüne S, Leser HG et al. Prospective, randomized, double blind trial of $p$ rophylaxis with single dose of co-amoxiclav before percutaneous endoscopic gastrostomy. BMJ 1999; 319:881-884.

283. Panigrahi H, Shreeve DR, Tan WC et al. Role of antibiotic prophylaxis for wound infection in percutaneous endoscopic gastrostomy (PEG): result of a prospective double-blind randomized trial. I Hosp Infect 2002; 50:312-315.

284. Sturgis TM, Yancy W, Cole JC et al. Antibiotic prophylaxis in percutaneous endoscopic gastrostomy. Am J Gastroenterol 1996; 91:2301-2304.

285. Banerjee S, Shen B, Baron TH, for the American Society for Gastrointestinal Endoscopy Standards of Practice Committee. Antibiotic prophylaxis for GI endoscopy. Gastrointest Endosc 2008; 67:791-798.

286. Rey JR, Axone A, Budzynska A et al. Guidelines of the European Society of Gastrointestinal Endoscopy (E.S.G.E.) antibiotic prophylaxis for gastrointestinal endoscopy. Endoscopy 1998; 30:318-324.

287. Allison MC, Sandoe JA, Tighe R et al., for the Endoscopy Committee of the British Society of Gastroenterology. Antibiotic prophylaxis in gastrointestinal endoscopy. Gut 2009; 58:869-880.

288. Gossner L, Keymling J, Hahn EG et al. Antibiotic prophylaxis in percutaneous endoscopic gastrostomy (PEG): a prospective randomized clinical trial. Endoscopy 1999; 31:119-124.

289. Rao GG, Osman M, Johnson L et al. Prevention of percutaneous endoscopic gastrostomy site infections caused by methicillin-resistant Staphylococcus aureus. J Hosp Infect 2004; 58:81-83.

290. Thomas S, Cantrill S, Waghorn DJ et al. The role of screening and antibiotic prophylaxis in the prevention of percutaneous gastrostomy site infection caused by methicillin-resistant Staphylococcus aureus. Aliment Pharmacol Ther 2007; 25:593-597.

291. Sauerland S, Angrisani L, Belachew M et al. Obesity surgery. Evidence-based guidelines of the European Association for Endoscopic Surgery (EAES). Surg Endosc 2005; 19:200-221.

292. Varela JE, Wilson SE, Nguyen NT. Laparoscopic surgery significantly reduces surgical-site infections compared with open surgery. Surg Endosc 2010; 24:270-276.

293. Ueno T, Yamamoto K, Kawaoka T et al. Current antibiotic prophylaxis in pancreatoduodenectomy in Japan. J Hepatobiliary Pancreat Surg 2005; 12:304-309.

294. Stone HH, Haney BH, Kolb LD et al. Prophylactic and preventive antibiotic therapy. Timing, duration and economics. Ann Surg 1979; 189:691-699.

295. Kusachi S, Sumiyama Y, Nagao J et al. Prophylactic antibiotics given within 24 hours of surgery, compared with antibiotics given for 72 hours perioperatively, increased the rate of methicillin-resistant Staphylococcus aureus isolated from surgical site infections. I Infect Chemother 2008; $14: 44-50$.

296. Imamura H, Furukawa H, Iijima S et al. Multicenter phase II study of antimicrobial prophylaxis in low-risk patients undergoing distal gastrectomy for gastric cancer. Gastric Cancer 2006; 9:32-35.

297. Alexander JW, Rahn R. Prevention of deep wound infection in morbidly obese patients by infusion of an antibiotic into the subcutaneous space at the time of wound closure. Obes Surg 2004; 14:970-974.

298. Radhakrishnan NV, Shenoy AH, Cartmill I et al. Addition of local antiseptic spray to parenteral antibiotic regimen reduces the incidence of stomal infection following percutaneous endoscopic gastrostomy: a randomized controlled trial. Eur J Gastroenterol Hepatol 2006; 18:12791284 . 
299. Bates T, Roberts JV, Smith K et al. A randomized trial of one versus three doses of augmentin as wound prophylaxis in at-risk abdominal surgery. Postgrad Med J 1992; 68:811-816.

300. McArdle CS, Morran CG, Anderson JR et al. Oral ciprofloxacin as prophylaxis in gastroduodenal surgery. $\underline{\mathrm{JH} \text { Hosp }}$ Infect 1995; 30:211-216.

301. Rawat D, Srivistava A, Thomson M. Antibody prophylaxis for children undergoing percutaneous endoscopic gastrostomy. J Pediatr Gastroenterol Nutr 2005; 40:234235.

302. Cortes A, Sauvanet A, Bert F et al. Effect of bile contamination on immediate outcomes after pancreaticoduodenectomy for tumor. J Am Coll Surg 2006; 202:93-99.

303. Solomkin JS, Mazuski JE, Bradley JS et al. Diagnosis and management of complicated intra-abdominal infection in adults and children: guidelines by the Surgical Infection Society and the Infectious Diseases Society of America. Surg Infect 2010; 11:79-109.

304. Meijer WS, Schmitz PI, Jeekel J. Meta-analysis of randomized, controlled clinical trials of antibiotic prophylaxis in biliary tract surgery. Br J Surg 1990; 77:282-290.

305. Den Hoed PT, Boelhouwer RU, Veen HF et al. Infections and bacteriological data after laparoscopic and open gallbladder surgery. J Hosp Infect 1998; 39:27-37.

306. Dervisoglou A, Tsiodras S, Kanellakopoulou K et al. The value of chemoprophylaxis against Enterococcus species in elective cholecystectomy: a randomized study of cefuroxime vs ampicillin/sulbactam. Arch Surg 2006; 141:11621167.

307. Cainzos M, Sayek I, Wacha H et al. Septic complications after biliary tract stone surgery: a review and report of the European Prospective Study. Hepatogastroenterology 1997; 44:959-967.

308. Lippert H, Gastinger J. Antimicrobial prophylaxis in laparoscopic and conventional cholecystectomy. Chemotherapy 1998; 44:355-363.

309. Siddiqui K, Khan AF. Comparison of frequency of wound infection: open vs laparoscopic cholecystectomy. J Ayub Med Coll Abbottabad 2006; 18:21-24.

310. Romy S, Eisenring MC, Bettschart V et al. Laparoscope use and surgical site infections in digestive surgery. Ann Surg 2008; 247:627-632.

311. Rotermann M. Infection after cholecystectomy, hysterectomy or appendectomy. Health Rep 2004; 15:11-23.

312. Chang WT, Lee KT, Chuang SC et al. The impact of prophylactic antibiotics on postoperative infection complication in elective laparoscopic cholecystectomy: a prospective randomized study. Am J Surg 2006; 191:721-725.

313. Zhou H, Zhang J, Wang Q et al. Meta-analysis: antibiotic prophylaxis in elective laparoscopic cholecystectomy. Aliment Pharmacol Ther 2009; 29:1086-1095.

314. Dobay KJ, Freier DT, Albear P. The absent role of prophylactic antibiotics in low-risk patients undergoing laparoscopic cholecystectomy. Am Surg 1999; 65:226-228.

315. Higgins A, London J, Charland S et al. Prophylactic antibiotics for elective laparoscopic cholecystectomy: are they necessary? Arch Surg 1999; 134:611-614.

316. Tocchi A, Lepre L, Costa G et al. The need for antibiotic prophylaxis in elective laparoscopic cholecystectomy: a prospective randomized study. Arch Surg 2000; 135: 67-70.

317. Guzmán-Valdivia G. Routine administration of antibiotics to patients suffering accidental gallbladder per- foration during laparoscopic cholecystectomy is not necessary. Surg Laparosc Endosc Percutan Tech 2008; 18: 547-550.

318. Harling R, Moorjani N, Perry C et al. A prospective, randomized trial of prophylactic antibiotics versus bag extraction in the prophylaxis of wound infection in laparoscopic cholecystectomy. Ann R Coll Surg Engl 2000; 82:408-410.

319. Illig KA, Schmidt E, Cavanaugh J et al. Are prophylactic antibiotics required for elective laparoscopic cholecystectomy? J Am Coll Surg 1997; 184:353-356.

320. Zurbuchen U, Ritz JP, Lehmann KS et al. Oral vs intravenous antibiotic prophylaxis in elective laparoscopic cholecystectomy - an exploratory trial. Langenbecks Arch Surg 2008; 393:479-485.

321. Al-Abassi AA, Farghaly MM, Ahmed HL et al. Infection after laparoscopic cholecystectomy: effect of infected bile and infected gallbladder wall. Eur J Surg 2001; 167:268-273.

322. Farello GA, Cerofolini A. Antimicrobial prophylaxis with ceftriaxone in laparoscopic cholecystectomy: a 7-year clinical experience involving 3,603 patients. I Chemother 2000; 12(suppl 3):17-22.

323. McGuckin M, Shea JA, Schwartz JS. Infection and antimicrobial use in laparoscopic cholecystectomy. Infect Control Hosp Epidemiol 1999; 20:624-626.

324. Biscione FM, Couto RC, Pedrosa TM et al. Comparison of the risk of surgical site infection after laparoscopic cholecystectomy and open cholecystectomy. Infect Control Hosp Epidemiol 2007; 28:1103-1106.

325. Brill A, Ghosh K, Gunnarsson C et al. The effects of laparoscopic cholecystectomy, hysterectomy, and appendectomy on nosocomial infection risks. Surg Endosc 2008; 22:1112-1118.

326. Cainzos M, Potel J, Puente JL. Prospective, randomized, controlled study of prophylaxis with cefamandole in highrisk patients undergoing operations upon the biliary tract. Surg Gynecol Obstet 1985; 160:27-32.

327. Meijer WS, Schmitz PI. Prophylactic use of cefuroxime in biliary tract surgery: randomized, controlled trial of single versus multiple dose in high-risk patients. Br J Surg 1993; 80:917-921.

328. Grant MD, Jones RC, Wilson SE et al. Single-dose cephalosporin prophylaxis in high-risk patients undergoing surgical treatment of the biliary tract. Surg Gynecol Obstet 1992; 174:347-354.

329. Lapointe RW, Roy AF, Turgeon PL et al. Comparison of single-dose cefotetan and multidose cefoxitin as intravenous prophylaxis in elective, open biliary tract surgery: a multicentre, double-blind, randomized study. Can J Surg 1994; 37:313-318.

330. Strachan CJ, Black J, Powis SJ et al. Prophylactic use of cephazolin against wound sepsis after cholecystectomy. Br Med J 1977; 1:1254-1256.

331. Berne TV, Yellin AE, Appleman MD et al. Controlled comparison of cefmetazole with cefoxitin for prophylaxis in elective cholecystectomy. Surg Gynecol Obstet 1990; 170:137-140.

332. Wilson SE, Hopkins JA, Williams RA. A comparison of cefotaxime versus cefamandole in prophylaxis for surgical treatment of the biliary tract. Surg Gynecol Obstet 1987; 164:207-212.

333. Kujath P. Antibiotic prophylaxis in biliary tract surgery. Ciprofloxacin versus ceftriaxone. Am J Med 1989; 87(5A): 255S-257S. 
334. Garibaldi RA, Skolnck D, Maglio S et al. Postcholecystectomy wound infection. Ann Surg 1986; 204: 650-654.

335. Levi JU, Martinez OV, Hutson DG et al. Ampicillin versus cefamandole in biliary tract surgery. Am Surg 1984; 50: 412-417.

336. Kellum JM, Duma RJ, Gorbach SL et al. Single-dose antibiotic prophylaxis for biliary surgery. Arch Surg 1987; 122:918-922.

337. Muller EL, Pitt HA, Thompson JE et al. Antibiotics in infections of the biliary tract. Surg Gynecol Obstet 1987; 165:285-292.

338. Jewesson PJ, Stiver G, Wai A et al. Double-blind comparison of cefazolin and ceftizoxime for prophylaxis against infections following elective biliary tract surgery. Antimicrob Agents Chemother 1996; 40:70-74.

339. Montravers P, Lepape A, Dubreuil L et al. Clinical and microbiological profiles of community-acquired and nosocomial intra-abdominal infections: results of the French prospective, observational EBIIA study. I Antimicrob Chemother 2009; 63:785-794.

340. Baquero F, Hsueh PR, Paterson DL et al. In vitro susceptibilities of aerobic and facultatively anaerobic gramnegative bacilli isolated from patients with intra-abdominal infections worldwide: 2005 results from Study for Monitoring Antimicrobial Resistance Trends (SMART). Surg Infect 2009; 10:99-104.

341. Chow JW, Satishchandran V, Snyder TA et al. In vitro susceptibilities of aerobic and facultative gram-negative bacilli isolated from patients with intra-abdominal infections worldwide: the 2002 Study for Monitoring Antimicrobial Resistance Trends (SMART). Surg Infect 2005; 6:439-448.

342. Choudhary A, Bechtold ML, Puli SR et al. Role of prophylactic antibiotics in laparoscopic cholecystectomy: a meta-analysis. J Gastrointest Surg 2008; 12:1847-1853.

343. Stone $\mathrm{HH}$, Hooper CA, Kolb LD et al. Antibiotic prophylaxis in gastric, biliary and colonic surgery. Ann Surg 1976; 184:443-452.

344. Sirinek KR, Schauer PR, Yellin AE et al. Single-dose cefuroxime versus multiple-dose cefazolin as prophylactic therapy for high-risk cholecystectomy. I Am Coll Surg 1994; 178:321-325.

345. Drumm J, Donovan IA, Wise R. A comparison of cefotetan and cefazolin for prophylaxis against wound infection after elective cholecystectomy. I Hosp Infect 1985; 6: 277-280.

346. Crenshaw CA, Glanges E, Webber CE et al. A prospective, randomized, double-blind study of preventive cefamandole therapy in patients at high risk for undergoing cholecystectomy. Surg Gynecol Obstet 1981; 153:546-552.

347. Leaper DJ, Cooper MJ, Turner A. A comparison trial between cefotetan and cephazolin for wound sepsis prophylaxis during elective upper gastrointestinal surgery with an investigation of cefotetan penetration into the obstructed biliary tree. J Hosp Infect 1986; 7:269-276.

348. Maki DG, Lammers JL, Aughey DR. Comparative studies of multiple-dose cefoxitin vs. single-dose cefonicid for surgical prophylaxis in patients undergoing biliary tract operations or hysterectomy. Rev Infect Dis 1984; 6(suppl 4):S887-S895.

349. Garcia-Rodriquez JA, Puig-LaCalle J, Arnau C et al. Antibiotic prophylaxis with cefotaxime in gastroduodenal and biliary surgery. Am J Surg 1989; 158:428-432.
350. Targarona EM, Garau J, Munoz-Ramos C et al. Singledose antibiotic prophylaxis in patients at high risk for infection in biliary surgery: a prospective and randomized study comparing cefonicid with mezlocillin. Surgery 1990; 107:327-334.

351. Krige JE, Isaacs S, Stapleton GN et al. Prospective, randomized study comparing amoxicillin-clavulanic acid and cefamandole for the prevention of wound infection in high-risk patients undergoing elective biliary surgery. J Hosp Infect 1992; 22(suppl A):33-41.

352. Agrawal CS, Sehgal R, Singh RK et al. Antibiotic prophylaxis in elective cholecystectomy: a randomized, double blind study comparing ciprofloxacin and cefuroxime. Indian J Physiol Pharmacol 1999; 43:501-504.

353. Kellum JM, Gargano S, Gorbach SL et al. Antibiotic prophylaxis in high-risk biliary operations: multicenter trial of single preoperative ceftriaxone versus multidose cefazolin. Am J Surg 1984; 148:15-21.

354. Tonelli F, Mazzei T, Novelli A et al. Amoxicillin/clavulanic acid versus cefotaxime for antimicrobial prophylaxis in abdominal surgery: a randomized trial. I Chemother 2002; 14:366-372.

355. McLeish AR, Keighley MR, Bishop HM. Selecting patients requiring antibiotics in biliary surgery by immediate Gram stains of bile at surgery. Surgery 1977; 81:473-477.

356. Krajden S, Yaman M, Fuksa M et al. Piperacillin versus cefazolin given perioperatively to high-risk patients who undergo open cholecystectomy: a double-blind, randomized trial. Can J Surg 1993; 36:245-250.

357. McArdle CS. Oral prophylaxis in biliary tract surgery. J Antimicrob Chemother 1994; 33:200-202.

358. Plouffe JF, Perkins RL, Fass RJ et al. Comparison of the effectiveness of moxalactam and cefazolin in the prevention of infection in patients undergoing abdominal operations. Diagn Microbiol Infect Dis 1985; 3:25-31.

359. Katz S, Glicksman A, Levy Y et al. Cefuroxime prophylaxis in biliary surgery: single versus triple dose. Israel J Med Sci 1993; 29:673-676.

360. Ahmed ME, Ibrahim SZ, Arabi YE et al. Metronidazole prophylaxis in acute mural appendicitis: failure of a single intra-operative infusion to reduce wound infection. J Hosp Infect 1987; 10:260-264.

361. Donovan IA, Ellis D, Gatehouse D et al. One-dose antibiotic prophylaxis against wound infection after appendectomy: a randomized trial of clindamycin, cefazolin sodium and a placebo. Br J Surg 1979; 66:193-196.

362. Gilmore OJ, Martin TD. Aetiology and prevention of wound infection in appendectomy. Br J Surg 1974; 62:567572.

363. Keiser TA, Mackenzie RL, Feld LN. Prophylactic metronidazole in appendectomy: a double-blind controlled trial. Surgery 1983; 93:201-203.

364. Winslow RE, Rem D, Harley JW. Acute nonperforating appendicitis: efficacy of brief antibiotic prophylaxis. Arch Surg 1983; 118:651-655.

365. Tonz M, Schmid P, Kaiser G. Antibiotic prophylaxis for appendectomy in children: critical appraisal. World J Surg 2000; 24:995-998.

366. Wilson AP. Antibiotic prophylaxis and infection control measures in minimally invasive surgery. J Antimicrob Chemother 1995; 36:1-5.

367. Aziz O, Athanasiou T, Tekkis PP. Laparoscopic versus open appendectomy in children: a meta-analysis. Ann Surg 2006; 243:17-27. 
368. Khan MN, Fayyad T, Cecil TD et al. Laparoscopic versus open appendectomy: the risk of postoperative infectious complications. JSLS 2007; 11:363-367.

369. Hansen J, Smithers MB, Schache D et al. Laparoscopic versus open appendectomy: prospective randomised trial. World J Surg 1996; 20:17-21.

370. Sauerland S, Lefering R, Neugebauer EA. Laparoscopic versus open surgery for suspected appendicitis. Cochrane Database Syst Rev 2004; 4:CD001546.

371. Hemmila MR, Birkmeyer NJ, Arbabi S et al. Introduction to propensity scores: a case study on the comparative effectiveness of laparoscopic vs open appendectomy. Arch Surg 2010; 145:939-945.

372. Stone HH. Bacterial flora of appendicitis in children. I Pediatr Surg 1976; 11:37-42.

373. Keighley MR. Infection: prophylaxis. Br Med Bull 1988; 44:374-402.

374. Andersen BR, Kallehave FL, Andersen HK. Antibiotics versus placebo for prevention of postoperative infection after appendicectomy. Cochrane Database Syst Rev 2005; 3:CD001439.

375. Helmer KS, Robinson EK, Lally KP et al. Standardized patient care guidelines reduce infectious morbidity in appendectomy patients. Am J Surg 2002; 183:608-613.

376. Lau WY, Fan ST, Yiu TF et al. Prophylaxis of postappendectomy sepsis by metronidazole and cefotaxime: a randomized, prospective and double-blind trial. $\underline{\mathrm{Br} J \text { Surg }}$ 1983; 70:670-672.

377. Lau WY, Fan ST, Chu KW et al. Randomized, prospective, and double-blind trial of new beta-lactams in the treatment of appendicitis. Antimicrob Agents Chemother 1985; 28:639-642.

378. Lau WY, Fan ST, Chu KW et al. Cefoxitin versus gentamicin and metronidazole in prevention of post-appendectomy sepsis: a randomized, prospective trial. I Antimicrob Chemother 1986; 18:613-619.

379. O’Rourke MG, Wynne MJ, Morahan RJ et al. Prophylactic antibiotics in appendectomy: a prospective, double-blind, randomized study. Aust N Z J Surg 1984; 54:535-541.

380. Liberman MA, Greason KL, Frame $S$ et al. Single-dose cefotetan or cefoxitin versus multiple-dose cefoxitin as prophylaxis in patients undergoing appendectomy for acute nonperforated appendicitis. I Am Coll Surg 1995; 180: 77-80.

381. Salam IM, Abu Galala KH, el Ashaal YI et al. A randomized prospective study of cefoxitin versus piperacillin in appendicectomy. J Hosp Infect 1994; 26:133-136.

382. Lau WY, Fan ST, Yiu TF et al. Prophylaxis of postappendectomy sepsis by metronidazole and ampicillin: a randomized, prospective and double-blind trial. $\underline{\mathrm{Br} J \text { Surg }}$ 1983; 70:155-157.

383. Al-Dhohayan A, Al-Sebayl M, Shibl A et al. Comparative study of Augmentin versus metronidazole/gentamicin in the prevention of infections after appendectomy. Eur Surg Res 1993; 25:60-64.

384. Morris WT, Innes DB, Richardson RA et al. The prevention of post-appendicectomy sepsis by metronidazole and cefazolin: a controlled double-blind trial. Aust N Z J Surg 1980; 50:429-433.

385. Morris DL, Wilson SR, Pain J et al. A comparison of aztreonam/metronidazole and cefotaxime/metronidazole in elective colorectal surgery: antimicrobial prophylaxis must include gram-positive cover. I Antimicrob Chemother 1990; 25:673-678.
386. Mui LM, Ng CS, Wong SK et al. Optimum duration of prophylactic antibiotics in acute non-perforated appendicitis. Aust N Z J Surg 2005; 75; 425-428.

387. Kasatpibal N, Nørgaard M, Sørensen HT et al. Risk of surgical site infection and efficacy of antibiotic prophylaxis: a cohort study of appendectomy patients in Thailand. BMC Infect Dis 2006; 6:111.

388. Nadler EP, Gaines BA. The Surgical Infection Society guidelines on antimicrobial therapy for children with appendicitis. Surg Infect 2008; 9:75-83.

389. Kizilcan F, Tanyel FC, Buyukpamukcu N et al. The necessity of prophylactic antibiotics in uncomplicated appendicitis during childhood. J Pediatr Surg 1992; 27:586-588.

390. Soderquist-Elinder C, Hirsch K, Bergdahl S et al. Prophylactic antibiotics in uncomplicated appendicitis during childhood: a prospective randomized study. Eur J Pediatr Surg 1995; 5:282-285.

391. Browder W, Smith JW, Vivoda LM et al. Nonperforative appendicitis: a continuing surgical dilemma. I Infect Dis 1989; 159:1088-1094.

392. Emil S, Laberge JM, Mikhail P et al. Appendicitis in children: a ten-year update of therapeutic recommendations. J Pediatr Surg 2003; 38:236-242.

393. Leong G, Wilson J, Charlett A. Duration of operation as a risk factor for surgical site infection: comparison of English and US data. J Hosp Infect 2006; 63:255-262.

394. Walz MJ, Paterson CA, Seligowski JM et al. Surgical site infection following bowel surgery: a retrospective analysis of 1446 patients. Arch Surg 2006; 141:1014-1018.

395. Salim A, Teixeira PG, Inaba K et al. Analysis of 178 penetrating stomach and small bowel injuries. World J Surg 2008; 32:471-475.

396. Witzke JD, Kraatz JJ, Morken JM et al. Stapled versus hand sewn anastomoses in patients with small bowel injury: a changing perspective. J Trauma 2000; 49:660-666.

397. Kirkpatrick AW, Baxter KA, Simons RK et al. Intraabdominal complications after surgical repair of small bowel injuries: an international review. J Trauma 2003; 55:399-406.

398. Brundage SI, Jurkovich GJ, Hoyt DB et al. Stapled versus sutured gastrointestinal anastomoses in the trauma patient: a multicenter trial. I Trauma 2001; 51:1054-1061.

399. Hackam DJ, Ali J, Jastaniah SS. Effects of other intraabdominal injuries on the diagnosis, management, and outcome of small bowel trauma. J Trauma 2000; 49:606-610.

400. Guarino J, Hassett JM Jr, Luchette FA. Small bowel injuries: mechanisms, patterns, and outcome. I Trauma 1995; 39:1076-1080.

401. Schnuriger B, Inaba K, Eberle BM et al. Microbiological profile and antimicrobial susceptibility in surgical site infections following hollow viscus injury. J Gastrointest Surg 2010; 14:1304-1310.

402. Múñez E, Ramos A, Espejo TÁ et al. [Microbiology of surgical site infections in abdominal tract surgery patients]. Cir Esp 2011; 89:606-612. In Spanish.

403. Den Hartog D, Dur AH, Tuinebreijer WE et al. Open surgical procedures for incisional hernias. Cochrane Database Syst Rev 2008; 3:CD006438.

404. Sanchez-Manuel FJ, Lozano-García J, Seco-Gil JL. Antibiotic prophylaxis for hernia repair. Cochrane Database Syst Rev 2012; 2:CD003769.

405. Yin Y, Song T, Liao B et al. Antibiotic prophylaxis in patients undergoing open mesh repair of inguinal hernia: a meta-analysis. Am Surg 2012; 78:359-365. 
406. Goodney PP, Birkmeyer CM, Birkmeyer JD. Short-term outcomes of laparoscopic and open ventral hernia repair: a meta-analysis. Arch Surg 2002; 137: 1161-1165.

407. Sajid MS, Bokhari SA, Mallick AS et al. Laparoscopic versus open repair of incisional/ventral hernia: a metaanalysis. Am J Surg 2009; 197:64-72.

408. Kaafarani HM, Kaufman D, Reda D et al. Predictors of surgical site infection in laparoscopic and open ventral incisional herniorrhaphy. J Surg Res 2010; 163:229-234.

409. Itani KM, Hur K, Kim LT et al. Comparison of laparoscopic and open repair with mesh for the treatment of ventral incisional hernia: a randomized trial. Arch Surg 2010; 145:322-328.

410. Forbes SS, Eskicioglu C, McLeod RS et al. Meta-analysis of randomized controlled trials comparing open and laparoscopic ventral and incisional hernia repair with mesh. $\underline{\mathrm{Br}}$ I Surg 2009; 96:851-858.

411. Sanchez VM, Abi-Haidar YE, Itani KM. Mesh infection in ventral incisional hernia repair: incidence, contributing factors, and treatment. Surg Infect 2011; 12:205-209.

412. Itani KM, Wilson SE, Awad SS et al. Ertapenem versus cefotetan prophylaxis in elective colorectal surgery. $\underline{N}$ Engl J Med 2006; 335:2640-2651.

413. Bartlett S, Burton R. Effects of prophylactic antibiotics on wound infection after elective colon and rectal surgery. Am J Surg 1983; 145:300-309.

414. Burton RC. Postoperative wound infection in colon and rectal surgery. Br J Surg 1973; 60:363-368.

415. Baum M, Anish D, Chalmers T et al. A survey of clinical trials of antibiotic prophylaxis in colon surgery: evidence against further use of no treatment controls. $\mathrm{N}$ Engl J Med 1981; 305:795-799.

416. Coppa G, Eng K, Gouge T et al. Parenteral and oral antibiotics in elective colorectal surgery: a prospective randomized trial. Am J Surg 1983; 145:62-65.

417. Coppa G, Eng K. Factors involved in antibiotic selection in elective colon and rectal surgery. Surgery 1988; 104:853858.

418. Glenny AM, Song F. Antimicrobial prophylaxis in colorectal surgery. Qual Health Care 1999; 8:132-136.

419. Suding P, Jensen E, Abramson MA et al. Definitive risk factors for anastomotic leaks in elective open colorectal resection. Arch Surg 2008; 143:907-912.

420. Svensson LG. Prophylactic antimicrobial administration. $\underline{S}$ Afr J Surg 1985; 23:55-62.

421. Hojer H, Wetterfors J. Systemic prophylaxis with doxycycline in surgery of the colon and rectum. Ann Surg 1978; 187:362-368.

422. Kurz A, Sessler DI, Lenhardt R, for the Study of Wound Infection and Temperature Group. Perioperative normothermia to reduce the incidence of surgical-wound infection and shorten hospitalization. N Engl J Med 1996; 334:1209-1215.

423. Ata A, Lee J, Bestle SL et al. Postoperative hyperglycemia and surgical site infection in general surgery patients. Arch Surg 2010; 145:858-864.

424. McConnell YJ, Johnson PM, Porter GA. Surgical site infections following colorectal surgery in patients with diabetes: association with postoperative hyperglycemia. I Gastrointest Surg 2009; 13:508-515.

425. Nichols RL. Prophylaxis for intraabdominal surgery. Rev Infect Dis 1984; 6(suppl 1):S276-S282.
426. Nelson RL, Glenny AM, Song F. Antimicrobial prophylaxis for colorectal surgery. Cochrane Database Syst Rev 2009; 1:CD001181.

427. Clarke J, Condon R, Bartlett J et al. Preoperative oral antibiotics reduce septic complications of colon operations: results of a prospective randomized, double-blind clinical study. Ann Surg 1977; 186:251-259.

428. Nichols R, Broldo P, Condon P et al. Effect of preoperative neomycin-erythromycin intestinal preparation on the incidence of infectious complications following colon surgery. Ann Surg 1973; 178:453-462.

429. Stellato T, Danziger L, Gordon N et al. Antibiotics in elective colon surgery: a randomized trial of oral, systemic, and oral/systemic antibiotics for prophylaxis. Am Surg 1990; 56:251-254.

430. Petrelli N, Contre DC, Herrera L et al. A prospective randomized trial of perioperative prophylactic cefamandole in elective colorectal surgery for malignancy. Dis Colon Rectum 1988; 31:427-429.

431. Kling PA, Dahlgren S. Oral prophylaxis with neomycin and erythromycin in colorectal surgery; more proof for efficacy than failure. Arch Surg 1989; 124:705-707.

432. Lewis RT, Goodall RG, Marien B et al. Is neomycin necessary for bowel preparation in surgery of the colon? Oral neomycin plus erythromycin versus erythromycinmetronidazole. Dis Colon Rectum 1989; 32:265-270.

433. Wapnick S, Gunito R, Leveen $\mathrm{HH}$ et al. Reduction of postoperative infection in elective colorectal surgery with preoperative administration of kanamycin and erythromycin. Surgery 1979; 85:317-321.

434. Bartlett J, Condon R, Gorbach S et al. Veterans Administration Cooperative Study on bowel preparation for elective colorectal operations: impact of oral antibiotic regimen on colonic flora, wound irrigation cultures and bacteriology of septic complications. Ann Surg 1978; 188:249-254.

435. Wolff B, Beart R, Dozios R et al. A new bowel preparation for elective colon and rectal surgery: a prospective, randomized clinical trial. Arch Surg 1988; 123:895-900.

436. Gahhos FN, Richards GK, Hinchey EJ et al. Elective colon surgery: clindamycin versus metronidazole prophylaxis. Can J Surg 1982; 25:613-616.

437. Dion YM, Richards GK, Prentis JJ et al. The influence of oral metronidazole versus parenteral preoperative metronidazole on sepsis following colon surgery. Ann Surg 1980; 192:221-226.

438. Beggs FD, Jobanputra RS, Holmes JT. A comparison of intravenous and oral metronidazole as prophylactic in colorectal surgery. Br J Surg 1982; 69:226-227.

439. Goldring J, McNaught W, Scott A et al. Prophylactic oral antimicrobial agents in elective colonic surgery: a controlled trial. Lancet 1975; 2:997-999.

440. Washington J, Dearing W, Judd E et al. Effect of preoperative antibiotic regimen on development of infection after intestinal surgery. Ann Surg 1974; 180:567-572.

441. Peruzzo L, Savio S, De Lalla F. Systemic versus systemic plus oral chemoprophylaxis in elective colorectal surgery. Chemioterapia 1987; 6:601-603.

442. Willis A, Fergunson I, Jones P et al. Metronidazole in prevention and treatment of Bacteroides infections in elective colonic surgery. Br Med J 1977; 1:607-610.

443. Hagen TB, Bergan T, Liavag I. Prophylactic metronidazole in elective colorectal surgery. Acta Chir Scand 1980; 146:71-75. 
444. Song F, Glenny AM. Antimicrobial prophylaxis in colorectal surgery: a systematic review of randomized controlled trials. Br J Surg 1998; 85:1232-1241.

445. Kaiser A, Herrington J, Jacobs J et al. Cefoxitin vs erythromycin, neomycin and cefazolin in colorectal surgery: importance of the duration of the operative procedure. Ann Surg 1983; 198:525-530.

446. Weaver M, Burdon DW, Youngs DJ et al. Oral neomycin and erythromycin compared with single-dose systemic metronidazole and ceftriaxone prophylaxis in elective colorectal surgery. Am J Surg 1986; 151:437-442.

447. Keighley MR, Arabi Y, Alexander-Williams J et al. Comparison between systemic and oral antimicrobial prophylaxis in colorectal surgery. Lancet 1979; 1:894-897.

448. Lewis RT, Allan CM, Goodall RG et al. Are first-generation cephalosporins effective for antibiotic prophylaxis in elective surgery of the colon? Can J Surg 1983; 26:504507.

449. Condon RE, Bartlett JG, Nichols RL et al. Preoperative prophylactic cephalothin fails to control septic complications of colorectal operations: results of a controlled clinical trial. Am J Surg 1979; 137:68-74.

450. McDermott F, Polyglase A, Johnson W et al. Prevention of wound infection in colorectal resections by preoperative cephazolin with and without metronidazole. Aust N Z J Surg 1981; 51:351-353.

451. Jones RN, Wojeski W, Bakke J et al. Antibiotic prophylaxis to 1,036 patients undergoing elective surgical procedures. A prospective randomized comparative trial of cefazolin, cefoxitin, and cefotaxime in a prepaid medical practice. Am J Surg 1987; 153:341-346.

452. Morton A, Taylor E, Wells G. A multicenter study to compare cefotetan alone with cefotetan and metronidazole as prophylaxis against infection in elective colorectal operations. Surg Gynecol Obstet 1989; 169:41-45.

453. Hoffman C, McDonald P, Watts J. Use of perioperative cefoxitin to prevent infection after colonic and rectal surgery. Ann Surg 1981; 193:353-356.

454. Periti P, Mazzei T, Tonelli F. Single-dose cefotetan vs multiple dose cefoxitin. Rectum 1989; 32:121-127.

455. Jagelman D, Fabian T, Nichols R et al. Single-dose cefotetan versus multiple dose cefoxitin as prophylaxis in colorectal surgery. Am J Surg 1988; 155:71-76.

456. Shatney $\mathrm{CH}$. Antibiotic prophylaxis in elective gastrointestinal tract surgery: a comparison of single-dose preoperative cefotaxime and multiple-dose cefoxitin. I Antimicrob Chemother 1984; 14(suppl B):241-245.

457. Arnaud JP, Bellissant E, Boissel P et al. Single-dose amoxycillin-clavulanic acid vs cefotetan for prophylaxis in elective colorectal surgery: a multicentre, prospective, randomized study. J Hosp Infect 1992; 22(suppl A):23-32.

458. Periti P, Tonelli F, Mazzei $\mathrm{T}$ et al. Antimicrobial chemoimmunoprophylaxis in colorectal surgery with cefotetan and thymostimulin: prospective, controlled, multicenter study. I Chemother 1993; 5:37-42.

459. Skipper D, Karran SJ. A randomized, prospective study to compare cefotetan with cefuroxime plus metronidazole as prophylaxis in elective colorectal surgery. J Hosp Infect 1992; 21:73-77.

460. Lumley JW, Siu SK, Pillay SP et al. Single-dose ceftriaxone as prophylaxis for sepsis in colorectal surgery. Aust N Z J Surg 1992; 62:292-296.

461. Hakansson T, Raahave D, Hansen $\mathrm{OH}$ et al. Effectiveness of single-dose prophylaxis with cefotaxime and metroni- dazole compared with three doses of cefotaxime alone in elective colorectal surgery. Eur J Surg 1993; 159:177-180.

462. Karran SJ, Sutton G, Gartell P et al. Imipenem prophylaxis in elective colorectal surgery. Br J Surg 1993; 80:1196-1198.

463. Fukatsu K, Saito H, Matsuda $T$ et al. Influences of type and duration of antimicrobial prophylaxis on an outbreak of methicillin-resistant Staphylococcus aureus and on the incidence of wound infection. Arch Surg 1997; 132:1320-1325.

464. AhChong K, Yip AW, Lee FC et al. Comparison of prophylactic ampicillin/sulbactam with gentamicin and metronidazole in elective colorectal surgery: a randomized clinical study. J Hosp Infect 1994; 27:149-154.

465. Kwok SP, Lau WY, Leung KL et al. Amoxicillin and clavulanic acid versus cefotaxime and metronidazole as antibiotic prophylaxis in elective colorectal resectional surgery. Chemotherapy 1993; 39:135-139.

466. Barbar MS, Hirxberg BC, Rice C et al. Parenteral antibiotics in elective colon surgery? A prospective, controlled clinical study. Surgery 1979; 86:23-29.

467. Madsen M, Toftgaard C, Gaversen $\mathrm{H}$ et al. Cefoxitin for one day vs ampicillin and metronidazole for three days in elective colorectal surgery: a prospective, randomized, multicenter study. Dis Colon Rectum 1988; 31:774-777.

468. Blair J, McLeod R, Cohen Z et al. Ticarcillin/clavulanic acid (Timentin) compared to metronidazole/netilmicin in preventing postoperative infection after elective colorectal surgery. Can J Surg 1987; 30:120-122.

469. Mendes Da Costa P, Kaufman L. Amikacin once daily plus metronidazole versus amikacin twice daily plus metronidazole in colorectal surgery. Hepatogastroenterology 1992; 39:350-354.

470. Roland M. Prophylactic regimens in colorectal surgery: an open randomized consecutive trial of metronidazole used alone or in combination with ampicillin or doxycycline. World J Surg 1986; 10:1003-1008.

471. Juul PZ, Klaaborg KE, Kronborg O. Single or multiple doses of metronidazole and ampicillin in elective colorectal surgery: a randomized trial. Dis Colon Rectum 1987; 30:526-528.

472. University of Melbourne Colorectal Group. A comparison of single-dose Timentin with mezlocillin for prophylaxis of wound infection in elective colorectal surgery. Dis Colon Rectum 1989; 32:940-943.

473. Stewart M, Taylor EW, Lindsay G. Infection after colorectal surgery: a randomized trial of prophylaxis with piperacillin versus sulbactam/piperacillin. J Hosp Infect 1995; 29:135-142.

474. Bergman L, Solhaug JH. Single-dose chemoprophylaxis in elective colorectal surgery. Ann Surg. 1987; 205:77-81.

475. Goransson G, Nilsson-Ehle I, Olsson S et al. Single-versus multiple-dose doxycycline prophylaxis in elective colorectal surgery. Acta Chir Scand 1984; 150:245-249.

476. Andaker L, Burman LG, Eklund A et al. Fosfomycin/ metronidazole compared with doxycycline/metronidazole for the prophylaxis of infection after elective colorectal surgery: a randomized, double-blind, multicentre trial in 517 patients. Eur J Surg 1992; 158:181185.

477. Sexton DJ. Carbapenems for surgical prophylaxis? N Engl I Med 2006; 355:2693-2695.

478. Condon RE, Bartlett J, Greenlee $\mathrm{H}$ et al. Efficacy of oral and systemic antibiotic prophylaxis in colorectal operations. Arch Surg 1983; 118:496-502. 
479. Condon RE. Preoperative antibiotic bowel preparation. Drug Ther 1983; 83:29-37.

480. Hinchey E, Richards G, Lewis R et al. Moxalactam as single agent prophylaxis in the prevention of wound infection following colon surgery. Surgery 1987; 101:15-19.

481. Jagelman DG, Fazio VW, Lavery IC et al. A prospective, randomized, double-blind study of $10 \%$ mannitol mechanical bowel preparation combined with oral neomycin and short-term, perioperative, intravenous Flagyl as prophylaxis in elective colorectal resections. Surgery 1985; 98:861-865.

482. Lewis RT. Oral versus systemic antibiotic prophylaxis in elective colon surgery: a randomized study and metaanalysis send a message from the 1990s. Can J Surg 2002; 45:173-180.

483. Englesbe MJ, Brooks L, Kubus J et al. A statewide assessment of surgical site infection following colectomy: the role of oral antibiotics. Ann Surg 2010; 252:514-520.

484. Kobayashi M, Mohri Y, Tonouchi H et al. Randomized clinical trial comparing intravenous antimicrobial prophylaxis alone with oral and intravenous antimicrobial prophylaxis for the prevention of a surgical site infection in colorectal cancer surgery. Surg Today 2007; 37: 383-388.

485. Wren SM, Ahmed N, Jamal A et al. Preoperative oral antibiotics in colorectal surgery increase the rate of Clostridium difficile colitis. Arch Surg 2005; 140:752-756.

486. Krapohl GL, Phillips LR, Campbell DA et al. Bowel preparation for colectomy and risk of Clostridium difficile infection. Dis Colon Rectum 2011; 54:810-817.

487. Espin-Basany E, Sanchez-Garcia JL, Lopez-Cano M et al. Prospective, randomised study on antibiotic prophylaxis in colorectal surgery. Is it really necessary to use oral antibiotics? Int J Colorectal Dis 2005; 20:542-546.

488. Sondheimer JM, Sokol RJ, Taylor S et al. Safety, efficacy, and tolerance of intestinal lavage in pediatric patients undergoing diagnostic colonoscopy. J Pediatr 1991; 119:148152.

489. Tuggle DW, Hoelzer DJ, Tunell WP et al. The safety and cost-effectiveness of polyethylene glycol electrolyte solution bowel preparation in infants and children. J Pediatr Surg 1987; 22:513-515.

490. Weber RS, Callender DL. Antibiotic prophylaxis in cleancontaminated head and neck oncologic surgery. Ann Otol Rhinol Laryngol 1992; 101:16-20.

491. Avenia N, Sanguinetti A, Cirocchi R et al. Antibiotic prophylaxis in thyroid surgery: a preliminary multicentric Italian experience. Ann Surg Innov Res 2009; 3:10.

492. Johnson JT, Wagner RL. Infection following uncontaminated head and neck surgery. Arch Otolaryngol Head Neck Surg 1987; 113:368-369.

493. Saginur R, Odell PF, Poliquin JF. Antibiotic prophylaxis in head and neck cancer surgery. I Otolaryngol. 1988; 17: 78-80.

494. Mandell-Brown M, Johnson JT, Wagner RL. Costeffectiveness of prophylactic antibiotics in head and neck surgery. Otolaryngol Head Neck Surg 1984; 92:520 523.

495. Johnson JT, Yu VL, Myers EN et al. Efficacy of two thirdgeneration cephalosporins in prophylaxis for head and neck surgery. Arch Otolaryngol 1984; 110:224-227.

496. Callender DL. Antibiotic prophylaxis in head and neck oncologic surgery: the role of gram-negative coverage. Int I Antimicrob Agents 1999; 12(suppl 1):S21-S27.
497. Simo R, French G. The use of prophylactic antibiotics in head and neck oncological surgery. Curr Opin Otolaryngol Head Neck Surg 2006; 14:55-61.

498. Lotfi CJ, Cavalcanti Rde C, Costa e Silva AM et al. Risk factors for surgical-site infections in head and neck cancer surgery. Otolaryngol Head Neck Surg 2008; 138:74-80.

499. Liu SA, Tung KC, Shiao JY et al. Preliminary report of associated factors in surgical site infection after major head and neck neoplasm operations-does the duration of prophylactic antibiotic matter? J Laryngol Otol 2008; 122:403-408.

500. Sepehr A, Santos BJ, Chou C et al. Antibiotics in head and neck surgery in the setting of malnutrition, tracheotomy, and diabetes. Laryngoscope 2009; 119:549-553.

501. Coskun H, Erisen L, Basut O. Factors affecting wound infection rates in head and neck surgery. Otolaryngol Head Neck Surg 2000; 123:328-333.

502. Robbins KT, Favrot S, Hanna D et al. Risk of surgical site infection in patients with head and neck cancer. Head Neck 1990; 12:143-148.

503. Tabet JC, Johnson JT. Wound infection in head and neck surgery: prophylaxis, etiology and management. J Otolaryngol 1990; 19:197-200.

504. Girod DA, McCulloch TM, Tsue TT et al. Risk factors for complications in clean-contaminated head and neck surgical procedures. Head Neck 1995; 17:7-13.

505. Miles BA, Potter JK, Ellis E III. The efficacy of postoperative antibiotic regimens in the open treatment of mandibular fractures: a prospective randomized trial. J Oral Maxillofac Surg 2006; 64:576-582.

506. Penel N, Fournier C, Roussel-Delvallez M et al. Prognostic significance of surgical site infections following major head and neck cancer surgery: an open noncomparative prospective study. Support Care Cancer 2004; 12:634-639.

507. Lovato C, Wagner JD. Infection rates following perioperative prophylactic antibiotics versus postoperative extended regimen prophylactic antibiotics in surgical management of mandibular fractures. I Oral Maxillofac Surg 2009; 67:827-832.

508. Strauss M, Saccogna PW, Allphin AL. Cephazolin and metronidazole prophylaxis in head and neck surgery. I Laryngol Otol 1997; 111:631-634.

509. Simons JP, Johnson JT, Yu VL et al. The role of topical antibiotic prophylaxis in patients undergoing contaminated head and neck surgery with flap reconstruction. Laryngoscope 2001; 111:329-335.

510. Johnson JT, Kachman K, Wagner RL et al. Comparison of ampicillin/sulbactam versus clindamycin in the prevention of infection in patients undergoing head and neck surgery. Head Neck 1997; 19:367-371.

511. Skitarelić N, Morović M, Manestar D. Antibiotic prophylaxis in clean-contaminated head and neck oncological surgery. J Craniomaxillofac Surg 2007; 35:15-20.

512. Andrews PJ, East CA, Jayaraj SM et al. Prophylactic vs. postoperative antibiotic use in complex septorhinoplasty surgery: a prospective, randomized, single-blind trial comparing efficacy. Arch Facial Plast Surg 2006; 8:84-87.

513. Becker GD, Welch WD. Quantitative bacteriology of closed-suction wound drainage in contaminated surgery. Laryngoscope. 1990; 100:403-406.

514. Johnson JT, Yu VL. Role of aerobic gram-negative rods, anaerobes, and fungi in surgical site infection after head 
and neck surgery: implications for antibiotic prophylaxis. Head Neck 1989; 11:27-29.

515. Rubin J, Johnson JT, Wagner RL et al. Bacteriologic analysis of surgical site infection following major head and neck surgery. Arch Otolaryngol Head Neck Surg 1988; 114:969-972.

516. Brown BM, Johnson JT, Wagner RL. Etiologic factors in head and neck surgical site infections. Laryngoscope 1987; 97:587-590.

517. Penel N, Fournier C, Lefebvre D et al. Multivariate analysis of risk factors for surgical site infection in head and neck squamous cell carcinoma surgery with opening of mucosa. Study of 260 surgical procedures. Oral Oncol 2005; 41:294-303.

518. Brook I. Microbiology and management of post-surgical wounds infection in children. Pediatr Rehabil 2002; 5:171176.

519. Brook I. Microbiology and principles of antimicrobial therapy for head and neck infections. Infect Dis Clin N Am 2007; 21:355-391.

520. National Institute for Health and Clinical Excellence. Surgical site infection (clinical guideline 74) 2008. www .nice.org.uk/CG74 (accessed 2012 Dec 9).

521. Andreasen JO, Jensen SS, Schwartz O et al. A systematic review of prophylactic antibiotics in the surgical treatment of maxillofacial fractures. J Oral Maxillofac Surg 2006; 64:1664-1668.

522. O'Reilly BJ, Black S, Fernandes J et al. Is the routine use of antibiotics justified in adult tonsillectomy? J Laryngol Otol 2003; 117:382-385.

523. Lee WC, Duignan MC, Walsh RM et al. An audit of prophylactic antibiotic treatment following tonsillectomy in children. J Laryngol Otol 1996; 110:357-359.

524. Caniello M, Passerotti GH, Goto EY et al. Antibiotics in septoplasty: is it necessary? Braz J Otorhinolaryngol 2005; 71:734-738.

525. Dhiwakar M, Eng CY, Selvaraj S et al. Antibiotics to improve recovery following tonsillectomy: a systematic review. Otolaryngol Head Neck Surg 2006; 134:357-364.

526. Dhiwakar M, Clement WA, Supriya M et al. Antibiotics to reduce post-tonsillectomy morbidity. Cochrane Database Syst Rev 2008; 2:CD005607.

527. Fennessy BG, Harney M, O'Sullivan MJ et al. Antimicrobial prophylaxis in otorhinolaryngology/head and neck surgery. Clin Otolaryngol 2007; 32:204-207.

528. Seven H, Sayin I, Turgut S. Antibiotic prophylaxis in clean neck dissections. J Laryngol Otol 2004; 118:213-216.

529. Slattery WH III, Stringer SP, Cassisi NJ. Prophylactic antibiotic use in clean, uncontaminated neck dissection. Laryngoscope. 1995; 105:244-246.

530. Weber RS, Raad I, Frankenthaler R et al. Ampicillinsulbactam vs clindamycin in head and neck oncologic surgery. The need for gram-negative coverage. Arch Otolaryngol Head Neck Surg 1992; 118:1159-1163.

531. Johnson JT, Myers EN, Thearle PB et al. Antimicrobial prophylaxis for contaminated head and neck surgery. Laryngoscope 1984; 94:46-51.

532. Righi M, Manfredi R, Farneti G et al. Short-term versus long-term antimicrobial prophylaxis in oncologic head and neck surgery. Head Neck 1996; 18:399-404.

533. Rajan GP, Fergie N, Fischer U et al. Antibiotic prophylaxis in septorhinoplasty? A prospective, randomized study. Plast Reconstr Surg 2005; 116:1995-1998.
534. Abubaker AO, Rollert MK. Postoperative antibiotic prophylaxis in mandibular fractures: a preliminary randomized, double-blind, and placebo-controlled clinical study. I Oral Maxillofac Surg 2001; 59:1415-1419.

535. Avery CM, Ameerally P, Castling B et al. Infection of surgical wounds in the maxillofacial region and free flap donor sites with methicillin-resistant Staphylococcus aureus. Br J Oral Maxillofac Surg 2006; 44:217-221.

536. Gantz NM. Nosocomial central nervous system infections. In: Mayhall CG, ed. Hospital epidemiology and infection control. 3rd ed. Philadelphia: Lippincott Williams and Wilkins; 2004.

537. Narotam PK, van Dellen JR, du Trevou MD et al. Operative sepsis in neurosurgery: a method of classifying surgical case. Neurosurgery 1994; 34:409-416.

538. Kourbeti IS, Jacobs AV, Koslow M et al. Risk factors associated with postcraniotomy meningitis. Neurosurgery 2007; 60:317-326.

539. Rebuck JA, Murry KR, Rhoney DH et al. Infection related to intracranial pressure monitors in adults: analysis of risk factor and antibiotic prophylaxis. I Neurol Neurosurg Psychiatry 2000; 69:381-384.

540. Korinek AM, Baugnon T, Golmard JL et al. Risk factors for adult nosocomial meningitis after craniotomy: role of antibiotic prophylaxis. Neurosurgery 2006; 58:126-133.

541. Lietard C, Thébaud $\mathrm{V}$, Besson $\mathrm{G}$ et al. Risk factors for neurosurgical site infections: an 18-month prospective survey. J Neurosurg 2008; 109:729-734.

542. Korinek AM, for the French Study Group of Neurosurgical Infections SEHP C-CLIN Paris-Nord. Risk factors for neurosurgical site infections after craniotomy: a prospective multicenter study of 2944 patients. Neurosurgery 1997; 41:10731081.

543. Korinek AM, Golmard JL, Elcheick A et al. Risk factors for neurosurgical site infections after craniotomy: a critical reappraisal of antibiotic prophylaxis on 4578 patients. $\underline{\mathrm{Br} J}$ Neurosurg 2005; 19:155-162.

544. Valentini LG, Casali C, Chatenoud L et al. Surgical site infections after elective neurosurgery: a survey of 1747 patients. Neurosurgery 2007; 61:88-96.

545. Zabramski JM, Whiting D, Darouiche RO et al. Efficacy of antimicrobial-impregnated external ventricular drain catheters: a prospective, randomized controlled trial. I Neurosurg 2003; 98:725-730.

546. Biyani N, Grisaru-Soen G, Steinbok P et al. Prophylactic antibiotics in pediatric shunt surgery. Childs Nerv Syst 2006; 22:1465-1471.

547. Holloway KL, Smith KW, Wilberger JE et al. Antibiotic prophylaxis during clean neurosurgery: a large, multicenter study using cefuroxime. Clin Ther 1996; 18:84-94.

548. Whitby M, Johnson BC, Atkinson RL et al. The comparative efficacy of intravenous cefotaxime and trimethoprim/sulfamethoxazole in preventing infection after neurosurgery: a prospective, randomized study. $\mathrm{Br}$ J Neurosurg 2000; 14:13-18.

549. Govender ST, Nathoo N, van Dellen JR. Evaluation of an antibiotic-impregnated shunt system for the treatment of hydrocephalus. J Neurosurg 2003; 99:831-839.

550. Tacconelli E, Cataldo MA, Albanese A et al. Vancomycin versus cefazolin prophylaxis for cerebrospinal shunt placement in a hospital with a high prevalence of methicillinresistant Staphylococcus aureus. J Hosp Infect 2008; 69: 337-344. 
551. Wong GK, Poon WS, Lyon D et al. Cefepime vs ampicillin/sulbactam and aztreonam as antibiotic prophylaxis in neurosurgical patients with external ventricular drain: result of a prospective randomized controlled clinical trial. I Clin Pharm Ther 2006; 31:231-235.

552. Ragal BT, Browd SR, Schmidt RH. Surgical shunt infection: significant reduction when using intraventricular and systemic antibiotic agents. I Neurosurg 2006; 105: 242-247.

553. Sarguna P, Lakshmi V. Ventriculoperitoneal shunt infections. Indian J Med Microbiol 2006; 24:52-54.

554. Langley JM, Gravel D, Moore D et al. Study of cerebrospinal fluid shunt-associated infections in the first year following placement, by the Canadian Nosocomial Infection Surveillance Program. Infect Control Hosp Epidemiol 2009; 30:285-288.

555. Nisbet M, Briggs S, Ellis-Pegler R et al. Propionibacterium acnes: an under-appreciated cause of post-neurosurgical infection. J Antimicrob Chemother 2007; 60:1097-1103.

556. Conen A, Walti LN, Merlo A et al. Characteristics and treatment outcome of cerebrospinal fluid shunt-associated infections in adults: a retrospective analysis over an 11year period. Clin Infect Dis 2008; 47:73-82.

557. Barker FG II. Efficacy of prophylactic antibiotics against meningitis after craniotomy: a meta-analysis. Neurosurgery 2007; 60:887-894.

558. Haines SJ, Goodman ML. Antibiotic prophylaxis of postoperative neurosurgical wound infection. I Neurosurg 1982; 56:103-105.

559. Quartey GR, Polyzoidis K. Intraoperative antibiotic prophylaxis in neurosurgery: a clinical study. Neurosurgery 1981; 8:669-671.

560. Savitz MH, Katz SS. Prevention of primary wound infection in neurosurgical patients: a 10-year study. Neurosurgery 1986; 18:685-688.

561. Blomstedt GC, Kytta J. Results of a randomized trial of vancomycin prophylaxis in craniotomy. J Neurosurg 1988; 69:216-220.

562. Shapiro M, Wald U, Simchen E et al. Randomized clinical trial of intraoperative antimicrobial prophylaxis of infection after neurosurgical procedures. J Hosp Infect 1986; 8:283-295.

563. Watters WC 3rd, Baisden J, Bono CM et al. Antibiotic prophylaxis in spine surgery: an evidence-based clinical guideline for the use of prophylactic antibiotics in spine surgery. Spine J 2009; 9:142-146.

564. Wang EL, Prober CG, Hendrick BE. Prophylactic sulfamethoxazole and trimethoprim in ventriculoperitoneal shunt surgery. A double-blind, randomized, placebocontrolled trial. JAMA 1984; 251:1174-1177.

565. Blomstedt GC. Results in trimethoprim-sulfamethoxazole prophylaxis in ventriculostomy and shunting procedures. J Neurosurg 1985; 62:694-697.

566. Djindjian M, Fevrier MJ, Ottervbein G et al. Oxacillin prophylaxis in cerebrospinal fluid shunt procedures: results of a randomized, open study in 60 hydrocephalic patients. Surg Neurol 1986; 24:178-180.

567. Blum J, Schwarz M, Voth D. Antibiotic single-dose prophylaxis of shunt infections. Neurosurg Rev 1989; 12:239244.

568. Schmidt K, Gjerris F, Osgaard O et al. Antibiotic prophylaxis in cerebrospinal fluid shunting: a prospective randomized trial in 152 hydrocephalic patients. Neurosurgery $1985 ; 17: 1-5$.
569. Griebel R, Khan M, Tan L. CSF shunt complications: an analysis of contributory factors. Childs Nerv Syst 1985; 1:77-80.

570. Lambert M, MacKinnon AE, Vaishnav A. Comparison of two methods of prophylaxis against CSF shunt infection. Z Kinderchir 1984; 39(suppl):109-110.

571. Zentner J, Gilsbach J, Felder T. Antibiotic prophylaxis in cerebrospinal fluid shunting: a prospective randomized trial in 129 patients. Neurosurg Rev 1995; 18:169-172.

572. Haines SJ, Walters BC. Antibiotic prophylaxis for cerebrospinal fluid shunts: a meta-analysis. Neurosurgery 1994; 34:87-92.

573. Langley JM, LeBlanc JC, Drake J et al. Efficacy of antimicrobial prophylaxis in placement of cerebrospinal fluid shunts: meta-analysis. Clin Infect Dis 1993; 17:98103.

574. Borowski A, Littleton AG, Borkhuu B et al. Complications of intrathecal baclofen pump therapy in pediatric patients. J Pediatr Orthop 2010; 30:76-81.

575. Motta F, Buonaguro V, Stignani C. The use of intrathecal baclofen pump implants in children and adolescents: safety and complications in 200 consecutive cases. I Neurosurg 2007; 107(suppl):32-35.

576. Fjelstad AB, Hommelstad J, Sorteberg A. Infections related to intrathecal baclofen therapy in children and adults: frequency and risk factors. J Neurosurg Pediatr 2009; 4:487493.

577. Follett KA, Boortz-Marx RL, Drake JM et al. Prevention and management of intrathecal drug delivery and spinal cord stimulation system infections. Anesthesiology 2004; 100:1582-1594.

578. Ratilal BO, Costa J, Sampaio C. Antibiotic prophylaxis for surgical introduction of intracranial ventricular shunts. Cochrane Database Syst Rev 2006; 3:CD005365.

579. Arnaboldi L. Antimicrobial prophylaxis with ceftriaxone in neurosurgical procedures: a prospective study of 100 patients undergoing shunt operations. Chemotherapy 1996; 42:384-390.

580. Alleyne $\mathrm{CH}$, Hassan M, Zabramski JM. The efficacy and cost of prophylactic and periprocedural antibiotics in patients with external ventricular drains. Neurosurgery 2000; 47:1124-1129.

581. Shurtleff DB, Stuntz JT, Hayden PW. Experience with 1201 cerebrospinal fluid shunt procedures. Pediatr Neurosci 1985-1986; 12:49-57.

582. Walters BC, Goumnerova L, Hoffman HJ et al. A randomized, controlled trial of perioperative rifampin/trimethoprim in cerebrospinal fluid shunt surgery. Childs Nerv Syst 1992; 8:253-257.

583. Martin JA, Hamilton BE, Sutton PD et al. Births: final data for 2006. Natl Vital Stat Rep 2009; 57:1-104.

584. Obstetric and medical complications. In: American Academy of Pediatrics and American College of Obstetricians and Gynecologists. Guidelines for perinatal care. 6th ed. Elk Grove Village, IL: American Academy of Pediatrics; 2008:175-204.

585. Hofmeyr GJ, Smaill F. Antibiotic prophylaxis for cesarean section. Cochrane Database Syst Rev 2002; 3: CD000933.

586. Killian C, Graffunder EM, Vinciguerra T et al. Risk factors for surgical-site infections following cesarean section. Infect Control Hosp Epidemiol 2001; 22:613-617.

587. Faro S. Infectious disease relations to cesarean section. Obstet Gynecol Clin North Am 1989; 16:363-371. 
588. Olsen MA, Butler AM, Willers DM et al. Risk factors for surgical site infection after low transverse cesarean section. Infect Control Hosp Epidemiol 2008; 29:477-484.

589. Hemsell DL. Infections after gynecologic surgery. Obstet Gynecol Clin North Am 1989; 16:381-400.

590. Faro S. Antibiotic prophylaxis. Obstet Gynecol Clin North Am 1989; 16:279-289.

591. Tita AT, Rouse DJ, Blackwell S et al. Emerging concepts in antibiotic prophylaxis for cesarean delivery: a systematic review. Obstet Gynecol 2009; 113:675-682.

592. Chelmow D, Ruehli MS, Huang E. Prophylactic use of antibiotics for nonlaboring patients undergoing cesarean delivery with intact membranes: a meta-analysis. Am J Obstet Gynecol 2001; 184:656-661.

593. Duff P, Smith PN, Keiser JF. Antibiotic prophylaxis in low-risk cesarean section. J Reprod Med 1982; 27:133138.

594. Apuzzio JJ, Reyelt C, Pelosi MA et al. Prophylactic antibiotics for cesarean section: comparison of high- and lowrisk patients for endometritis. Obstet Gynecol 1982; 59: 693-698.

595. Duff P. Prophylactic antibiotics for cesarean delivery: a simple cost-effective strategy for prevention of postoperative morbidity. Am J Obstet Gynecol 1987; 157:794-798.

596. Jakobi P, Weissman A, Sigler E et al. Post-cesarean section febrile morbidity. Antibiotic prophylaxis in low-risk patients. J Reprod Med 1994; 39:707-710.

597. Rizk DE, Nsanze H, Mabrouk MH et al. Systemic antibiotic prophylaxis in elective cesarean delivery. Int J Gynaecol Obstet 1998; 61:245-251.

598. Rouzi AA, Khalifa F, Ba'aqeel $\mathrm{H}$ et al. The routine use of cefazolin in cesarean section. Int J Gynecol Obstet 2000; 69:107-112.

599. Bagratee JS, Moodley J, Kleinschmidt I et al. A randomized controlled trial of antibiotic prophylaxis in elective caesarean delivery. Br J Obstet Gynaecol 2001; 108:143148.

600. Hopkins L, Smaill F. Antibiotic prophylaxis regimens and drugs for cesarean section. Cochrane Database Syst Rev 1999; 2:CD001136.

601. Andrews WW, Hauth JC, Cliver SP et al. Randomized clinical trial of extended spectrum antibiotic prophylaxis with coverage for Ureaplasma urealyticum to reduce postcesarean delivery endometritis. Obstet Gyncol 2003; 101:11831189.

602. Tita AT, Hauth JC, Grimes A et al. Decreasing incidence of postcesarean endometritis with extended-spectrum antibiotic prophylaxis. Obstet Gynecol 2008; 111:51-56.

603. Tita AT, Owen J, Stamm A et al. Impact of extendedspectrum antibiotic prophylaxis on incidence of postcesarean surgical wound infection. Am J Obstet Gynecol 2008; 199:303.e1-e3.

604. Meyer NL, Hosier KV, Scott K et al. Cefazolin versus cefazolin plus metronidazole for antibiotic prophylaxis at cesarean section. South Med J 2003; 96:992-995.

605. Costantine MM, Rahman M, Ghulmiyah L et al. Timing of perioperative antibiotics for cesarean delivery: a metaanalysis. Am J Obstet Gynecol 2008; 199:301.e1-e6.

606. Sullivan SA, Smith T, Chang E et al. Administration of cefazolin prior to skin incision is superior to cefazolin at cord clamping in preventing postcesarean infectious morbidity: a randomized, controlled trial. Am J Obstet Gynecol 2007; 196:455.e1-e5.
607. Committee Opinion No. 465: antimicrobial prophylaxis for cesarean delivery: timing of administration. Obstet Gynecol 2010; 116:791-792.

608. Witt A, Döner M, Petricevic L et al. Antibiotic prophylaxis before surgery vs after cord clamping in elective cesarean delivery: a double-blind, prospective, randomized, placebocontrolled trial. Arch Surg 2011; 146:1404-1409.

609. Whiteman MK, Hillis SD, Jamieson DJ et al. Inpatient hysterectomy surveillance in the United States, 2000-2004. Am J Obstet Gynecol 2008; 198:34.e1-e7.

610. Schorge JO, Schaffer JI, Halvorson LM et al. Surgeries for benign gynecologic conditions. Chap. 41. In: Schorge JO, Schaffer JI, Halvorson LM et al., eds. Williams gynecology. New York: McGraw-Hill Professional; 2008.

611. Drahonovsky J, Haakova L, Otcenasek M et al. A prospective randomized comparison of vaginal hysterectomy, laparoscopically assisted vaginal hysterectomy, and total laparoscopic hysterectomy in women with benign uterine disease. Eur J Obstet Gynecol 2009; 148:172-176.

612. Sokol AI, Green IC. Laparoscopic hysterectomy. Clin Obstet Gynecol 2009; 52:304-312.

613. ACOG Committee Opinion No. 444: choosing the route of hysterectomy for benign disease. Obstet Gynecol 2009; 114:1156-1158.

614. Nieboer TE, Johnson N, Lethaby A et al. Surgical approach to hysterectomy for benign gynaecological disease. Cochrane Database Syst Rev 2009; 3:CD003677.

615. American College of Obstetricians and Gynecologists. ACOG Committee Opinion No. 388: supracervical hysterectomy. Obstet Gynecol 2007; 110:1215-1217.

616. Jennings RH. Prophylactic antibiotics in vaginal and abdominal hysterectomy. South Med J 1978; 71:251-254.

617. Hemsell D, Johnson ER, Hemsell PG et al. Cefazolin is inferior to cefotetan as single-dose prophylaxis for women undergoing elective total abdominal hysterectomy. Clin Infect Dis 1995; 20:677-684.

618. Goosenberg J, Emich JP Jr, Schwarz RH. Prophylactic antibiotics in vaginal hysterectomy. Am J Obstet Gynecol 1969; 105:503-506.

619. Marsden DE, Cavanagh D, Wisniewski BJ et al. Factors affecting the incidence of infectious morbidity after radical hysterectomy. Am J Obstet Gynecol 1985; 152:817-821.

620. Mann W, Orr J, Shingleton H et al. Perioperative influences on infectious morbidity in radical hysterectomy. Gynecol Oncol 1981; 11:207-212.

621. Löfgren M, Poromaa IS, Stjerndahl JH et al. Postoperative infections and antibiotic prophylaxis for hysterectomy in Sweden: a study by the Swedish National Register for Gynecologic Surgery. Acta Obstet Gynecol Scand 2004; 83:12021207.

622. Olsen MA, Higham-Kessler J, Yokoe DS et al. Developing a risk stratification model for surgical site infection after abdominal hysterectomy. Infect Control Hosp Epidemiol 2009; 30:1077-1083.

623. Soper DE, Bump RC, Hurt GW. Wound infection after abdominal hysterectomy: effect of the depth of subcutaneous tissue. Am J Obstet Gynecol 1995; 173:465-471.

624. Soper DE. Infections of the female pelvis. In: Mandell GL, Bennett JE, Dolin R, eds. Mandell, Douglas and Bennett's principles and practice of infectious diseases. 7th ed. New York: Churchill Livingstone; 2009:1514-1516.

625. Ohm MJ, Galask RP. The effect of antibiotic prophylaxis on patients undergoing vaginal operations. II. Alterations 
of microbial flora. Am J Obstet Gynecol 1975; 123 597-604.

626. Ohm MJ, Galask RP. The effect of antibiotic prophylaxis on patients undergoing total abdominal hysterectomy. II. Alterations of microbial flora. Am J Obstet Gynecol 1976; 125:448-454.

627. Appelbaum P, Moodley J, Chatterton S et al. Metronidazole in the prophylaxis and treatment of anaerobic infection. S Afr Med J 1978; 1:703-706.

628. Mittendorf R, Aronson MP, Berry RE et al. Avoiding serious infections associated with abdominal hysterectomy: a meta-analysis of antibiotic prophylaxis. Am J Obstet Gynecol 1993; 169:1119-1124.

629. Duff P. Antibiotic prophylaxis for abdominal hysterectomy. Obstet Gynecol 1982; 60:25-29.

630. Miyazawa K, Hernandez E, Dillon MB. Prophylactic topical cefamandole in radical hysterectomy. Int J Gynaecol Obstet 1987; 25:133-138.

631. Micha JP, Kucera PR, Birkett JP et al. Prophylactic mezlocillin in radical hysterectomy. Obstet Gynecol 1987; 69:251254.

632. Sevin B, Ramos R, Lichtinger M et al. Antibiotic prevention of infection complicating radical abdominal hysterectomy. Obstet Gynecol 1984; 64:539-545.

633. Rosenshein NB, Ruth JC, Villar J et al. A prospective randomized study of doxycycline as a prophylactic antibiotic in patients undergoing radical hysterectomy. $\underline{\mathrm{Gy}}-$ necol Oncol 1983; 15:201-206.

634. Zakashansky K, Bradley WH, Nezhat FR. New techniques in radical hysterectomy. Curr Opin Obstet Gynecol 2008; 20:14-19.

635. Hemsell D, Menon M, Friedman A. Ceftriaxone or cefazolin prophylaxis for the prevention of infection after vaginal hysterectomy. Am J Surg 1984; 148:22-26.

636. Hemsell DL, Johnson ER, Bawdon RE et al. Ceftriaxone and cefazolin prophylaxis for hysterectomy. Surg Gynecol Obstet 1985; 161:197-203.

637. Soper D, Yarwood R. Single-dose antibiotic prophylaxis in women undergoing vaginal hysterectomy. Obstet Gynecol 1987; 53:879-882.

638. Rapp RP, Connors E, Hager WD et al. Comparison of single-dose moxalactam and a three-dose regimen of cefoxitin for prophylaxis in vaginal hysterectomy. Clin Pharm 1986; 5:988-993.

639. Roy S, Wilkins J. Single-dose cefotaxime versus 3- to 5-dose cefoxitin for prophylaxis of vaginal or abdominal hysterectomy. J Antimicrob Chemother 1984; 14(suppl B):217221.

640. Roy S, Wilkins J, Hemsell DL et al. Efficacy and safety of single-dose ceftizoxime vs. multiple-dose cefoxitin in preventing infection after vaginal hysterectomy. J Reprod Med 1988; 33(suppl 1):149-153.

641. Roy S, Wilkins J, Galaif E et al. Comparative efficacy and safety of cefmetazole or cefoxitin in the prevention of postoperative infection following vaginal and abdominal hysterectomy. J Antimicrob Chemother 1989; 23 (suppl D):109-117.

642. Mercer LJ, Murphy HJ, Ismail MA et al. A comparison of cefonicid and cefoxitin for preventing infections after vaginal hysterectomy. J Reprod Med 1988; 33:223-226.

643. Hemsell DL, Heard ML, Nobles BJ et al. Single-dose cefoxitin prophylaxis for premenopausal women undergoing vaginal hysterectomy. Obstet Gynecol 1984; 63:285290.
644. McGregor JA, Phillips LE, Dunne JT et al. Results of a double-blind, placebo controlled clinical trial program of single-dose ceftizoxime versus multiple-dose cefoxitin as prophylaxis for patients undergoing vaginal and abdominal hysterectomy. I Am Coll Surg 1994; 178:12-31.

645. Orr JW Jr, Varner RE, Kilgore LC et al. Cefotetan versus cefoxitin as prophylaxis in hysterectomy. Am J Obstet Gynecol 1986; 154:960-963.

646. Orr JW Jr, Sisson PF, Barrett JM et al. Single center study results of cefotetan and cefoxitin prophylaxis for abdominal or vaginal hysterectomy. Am J Obstet 1988; 158(3 pt 2):714-716

647. Berkeley AS, Orr JW, Cavanagh D et al. Comparative effectiveness and safety of cefotetan and cefoxitin as prophylactic agents in patients undergoing abdominal or vaginal hysterectomy. Am J Surg 1988; 155:81-85.

648. Berkeley AS, Freedman KS, Ledger WJ et al. Comparison of cefotetan and cefoxitin prophylaxis for abdominal and vaginal hysterectomy. Am J Obstet Gynecol 1988; 158: 706-709.

649. Gordon SF. Results of a single center study of cefotetan prophylaxis in abdominal or vaginal hysterectomy. Am J Obstet Gynecol 1988; 158:710-714. [Erratum, Am J Obstet Gynecol 1989; 160:1025.]

650. Campillo F, Rubio JM. Comparative study of single-dose cefotaxime and multiple doses of cefoxitin and cefazolin as prophylaxis in gynecologic surgery. Am J Surg 1992; 164(suppl):12S-15S.

651. Berkeley AS, Haywork SD, Hirsch JC et al. Controlled, comparative study of moxalactam and cefazolin for prophylaxis of abdominal hysterectomy. Surg Gynecol Obstet 1985; 161:457-461.

652. Tuomala RE, Fischer SG, Munoz A et al. A comparative trial of cefazolin and moxalactam as prophylaxis for preventing infection after abdominal hysterectomy. Obstet Gynecol 1985; 66:372-376.

653. Chongsomchai C, Lumbiganon $\mathrm{P}$, Thinkhamrop J et al. Placebo-controlled, double-blind, randomized study of prophylactic antibiotics in elective abdominal hysterectomy. J Hosp Infect 2002; 52:302-306.

654. Cormio G, Di Fazio F, Lorusso F et al. Antimicrobial prophylaxis in laparotomic gynecologic surgery: a prospective randomized study comparing amoxicillin-clavulanic acid with cefazolin. J Chemother 2002; 14:618-622.

655. Lett WJ, Ansbacher R, Davison BL et al. Prophylactic antibiotics for women undergoing vaginal hysterectomy. I Reprod Med 1977; 19:51-54.

656. Hamod KA, Spence MR, Roshenshein NB et al. Single and multidose prophylaxis in vaginal hysterectomy: a comparison of sodium cephalothin and metronidazole. Am J Obstet Gynecol 1980; 136:976-979.

657. Hemsell DL, Johnson ER, Heard MC et al. Single dose piperacillin versus triple dose cefoxitin prophylaxis at vaginal and abdominal hysterectomy. South Med J 1989; 82:438-442.

658. Turano A. New clinical data on the prophylaxis of infections in abdominal, gynecologic, and urologic surgery. Am J Surg 1992; 164(suppl):16S-20S.

659. D'Addato F, Canestrelli M, Repinto A et al. Perioperative prophylaxis in abdominal and vaginal hysterectomy. Clin Exp Obstet Gynecol 1993; 20:95-101.

660. Gonen R, Hakin M, Samberg I et al. Short-term prophylactic antibiotic for elective abdominal hysterectomy: how short? Eur J Obstet Gynecol Reprod Biol 1985; 20:229-234. 
661. Scarpignato C, Labruna C, Condemi V et al. Comparative efficacy of two different regimens of antibiotic prophylaxis in total abdominal hysterectomy. Pharmatherapeutica 1980; 2:450-455.

662. Hemsell DL, Hemsell PG, Heard ML et al. Preoperative cefoxitin prophylaxis for elective abdominal hysterectomy. Am J Obstet Gynecol 1985; 153:225-226.

663. Triolo O, Mancuso A, Pantano F. Amoxycillin/clavulanate prophylaxis in gynecologic surgery. Int J Gynaecol Obstet 2004; 85:59-61.

664. Su HY, Ding DC, Chen DC et al. Prospective randomized comparison of single-dose versus 1-day cefazolin for prophylaxis in gynecologic surgery. Acta Obstet Gynecol Scand 2005; 84:384-389.

665. Tchabo JG, Cutting ME, Butler C. Prophylactic antibiotics in patients undergoing total vaginal or abdominal hysterectomy. Int Surg 1985; 70:349-352.

666. Read RW. Endophthalmitis. In: Yanoff M, Duker JS, eds. Ophthalmology. 3rd ed. St. Louis: Mosby; 2009.

667. Bucci FA, Amico LM, Evans RE. Antimicrobial efficacy of prophylactic gatifloxacin $0.3 \%$ and moxifloxacin $0.5 \%$ in patients undergoing phacoemulsification surgery. Eye Contact Lens 2008; 34:39-42.

668. Colleaux KM, Hamilton WK. Effect of prophylactic antibiotics and incision type on the incidence of endophthalmitis after cataract surgery. Can J Ophthalmol 2000; 35:373-378.

669. Eifrig CW, Flynn HW Jr, Scott IU et al. Acute-onset postoperative endophthalmitis: review of incidence and visual outcomes (1995-2001). Ophthalmic Surg Lasers 2002; 33:373-378.

670. Garat M, Moser CL, Martín-Baranera M et al. Prophylactic intracameral cefazolin after cataract surgery: endophthalmitis risk reduction and safety results in a 6-year study. J Cataract Refract Surg 2009; 35:637-642.

671. Jensen MK, Fiscella RG, Moshirfar $M$ et al. Third- and fourth-generation fluoroquinolones: retrospective comparison of endophthalmitis after cataract surgery performed over 10 years. J Cataract Refract Surg 2008; 34:1460-1467.

672. Mollan SP, Mollan AJ, Konstantinos C et al. Incidence of endophthalmitis following vitreoretinal surgery. Int Ophthalmol 2009; 29:203-205.

673. Moshirfar M, Feiz V, Vitale AT et al. Endophthalmitis after uncomplicated cataract surgery with the use of fourth-generation fluoroquinolones: a retrospective observational case series. Ophthalmology 2007; 114:686-691.

674. Romero P, Méndez I, Salvat M et al. Intracameral cefazolin as prophylaxis against endophthalmitis in cataract surgery. I Cataract Refract Surg 2006; 32:438-441.

675. Soto AM, Mendívil MP. The effect of topical povidoneiodine, intraocular vancomycin, or both on aqueous humor cultures at the time of cataract surgery. Am J Ophthalmol 2001; 131:293-300.

676. Taban M, Behrens A, Newcomb RL et al. Incidence of acute endophthalmitis following penetrating keratoplasty. Arch Ophthalmol 2005; 123:605-609.

677. Wejde G, Samolov B, Seregard S et al. Risk factors for endophthalmitis following cataract surgery: a retrospective case-control study. J Hosp Infect 2005; 61:251-256.

678. Wu PC, Li M, Chang SJ et al. Risk of endophthalmitis after cataract surgery using different protocols for povidoneiodine preoperative disinfection. $\underline{\mathrm{J} \text { Ocul Pharmacol Ther }}$ 2006; 22:54-61.
679. Barry P, Seal DV, Gettinby G et al. ESCRS study of prophylaxis of postoperative endophthalmitis after cataract surgery: preliminary report of principal results from a European multicenter study. J Cataract Refract Surg 2006; 32:407-410.

680. ESCRS Endophthalmitis Study Group. Prophylaxis of postoperative endophthalmitis following cataract surgery: results of the ESCRS multicenter study and identification of risk factors. J Cataract Refract Surg 2007; 33:978-988.

681. Kamalarajah S, Ling R, Silvestri G et al. Presumed infectious endophthalmitis following cataract surgery in the UK: a case-control study of risk factors. Eye 2007; 21: 580-586.

682. Montan PG, Setterquist H, Marcusson E et al. Preoperative gentamicin eye drops and chlorhexidine solution in cataract surgery: experimental and clinical results. Eur J Ophthalmol 2000; 10:286-292.

683. Recchia FM, Busbee BG, Pearlman RB et al. Changing trends in the microbiologic aspects of postcataract endophthalmitis. Arch Ophthalmol 2005; 123:341-346.

684. American Academy of Ophthalmology. Cataract in the adult eye, preferred practice pattern. http://one.aao .org /CE/PracticeGuidelines / PPP_Content.aspx?cid = a80a87ce-9042-4677-85d7-4b876deed276 (accessed 2012 Dec 9).

685. Hatch WV, Cernat G, Wong D et al. Risk factors for acute endophthalmitis after cataract surgery: a populationbased study. Ophthalmology 2009; 116:425-440.

686. Lertsumitkul S, Myers PC, O'Rourke MT et al. Endophthalmitis in the western Sydney region: a casecontrol study. Clin Experiment Ophthalmol 2001; 29:400405.

687. De Kaspar HM, Chang RT, Singh $\mathrm{K}$ et al. Prospective randomized comparison of 2 different methods of $5 \%$ povidone-iodine applications for anterior segment intraocular surgery. Arch Ophthalmol 2005; 123:161-165.

688. Ta CN, Egbert PR, Singh K et al. Prospective randomized comparison of 3-day versus 1-hour preoperative ofloxacin prophylaxis for cataract surgery. Ophthalmology 2002; 109:2036-2041.

689. Fernández-Rubio E, Urcelay JL, Cuesta-Rodriguez T. The antibiotic resistance pattern of conjunctival bacteria: a key for designing a cataract surgery prophylaxis. Eye 2009; 23:1321-1328.

690. Gelfand YA, Mezer E, Linn S et al. Lack of effect of prophylactic gentamicin treatment on intraocular and extraocular fluid cultures after pars plana vitrectomy. Ophthalmic Surg Lasers 1998; 29:497-501.

691. Vasavada AR, Gajjar D, Raj SM et al. Comparison of 2 moxifloxacin regimens for preoperative prophylaxis: prospective randomized triple-masked trial. Part 2: residual conjunctival flora. I Cataract Refract Surg 2008; 34:1383-1388.

692. Osher RH, Amdahl LD, Cheetham JK. Antimicrobial efficacy and aqueous humor concentration of preoperative and postoperative topical trimethoprim/polymyxin B sulfate versus tobramycin. J Cataract Refract Surg 1994; 20:3-8.

693. Barequet IS, Jabbur NS, Barron Y et al. Perioperative microbiologic profile of the conjunctiva in photoreactive keratectomy. I Refract Surg 2001; 17:55-62.

694. Seal DV, Barry P, Gettinby G et al. ESCRS study of prophylaxis of postoperative endophthalmitis after cataract 
surgery: case for a European multicenter study. J Cataract Refract Surg 2006; 32:396-406.

695. Gore DM, Anjunawela RI, Little BC. United Kingdom survey of antibiotic prophylaxis practice after publication of the ESCRS endophthalmitis study. I Cataract Refract Surg 2009; 35:770-773.

696. O'Brien TP, Arshinoff SA, Mah FS. Perspective on antibiotics for postoperative endophthalmitis prophylaxis: potential role of moxifloxacin. J Cataract Refract Surg 2007; 33:1790-1800.

697. Chang DF, Braga-Mele R, Mamalis N et al. Prophylaxis of postoperative endophthalmitis after cataract surgery: results of the 2007 ASCRS member survey. J Cataract Refract Surg 2007; 33:1801-1805.

698. De Kaspar HM, Kreutzer TC, Aguirre-Romo I et al. A prospective randomized study to determine the efficacy of preoperative topical levofloxacin in reducing conjunctival bacterial flora. Am J Ophthalmol 2008; 145:136-142.

699. Montan PG, Wejde G, Setterquist H et al. Prophylactic intracameral cefuroxime: evaluation of safety and kinetics in cataract surgery. I Cataract Refract Surg 2002; 28:982987.

700. Chisari G, Cavallaro G, Reibaldi M et al. Presurgical antimicrobial prophylaxis: effect on ocular flora in healthy patients. Int J Clin Pharmacol Ther 2004; 42:35-38.

701. Park SH, Lim JA, Choi JS et al. The resistance patterns of normal ocular bacterial flora to 4 fluoroquinolone antibiotics. Cornea 2009; 28:68-72.

702. Ciulla TA, Starr MB, Masket S. Bacterial endophthalmitis prophylaxis for cataract surgery: an evidence-based update. Ophthalmology 2002; 109:13-26.

703. Gordon-Bennett P, Karas A, Flanagan D et al. A survey of measures used for the prevention of postoperative endophthalmitis after cataract surgery in the United Kingdom. Eye 2008; 22:620-627.

704. Ta CN, Singh K, Egbert PR et al. Prospective comparative evaluation of povidone-iodine $(10 \%$ for 5 minutes versus $5 \%$ for 1 minute) as prophylaxis for ophthalmic surgery. J Cataract Refract Surg 2008; 34:171-172.

705. Cahane M, Ben Simon GJ, Barequet IS et al. Human corneal stromal tissue concentration after consecutive doses of topically applied 3.3\% vancomycin. Br J Ophthalmol 2004; 88:22-24.

706. Bucci FA. An in vivo study comparing the ocular absorption of levofloxacin and ciprofloxacin prior to phacoemulsification. Am J Ophthalmol 2004; 137:308-312.

707. García-Sáenz MC, Arias-Puente A, Fresnadillo-Martinez MJ et al. Human aqueous humor levels of oral ciprofloxacin, levofloxacin and moxifloxacin. I Cataract Refract Surg 2001; 27:11969-11974.

708. Hariprasad SM, Blinder KJ, Shah GK et al. Penetration pharmacokinetics of topically administered $0.5 \%$ moxifloxacin ophthalmic solution in human aqueous and vitreous. Arch Ophthalmol 2005; 123:39-44.

709. Holland EJ, McCarthy M, Holland S. The ocular penetration of levofloxacin $1.5 \%$ and gatifloxacin $0.3 \%$ ophthalmic solutions in subjects undergoing corneal transplant surgery. Curr Med Res Opin 2007; 23:2955-2960.

710. Costello P, Bakri SJ, Beer PM et al. Vitreous penetration of topical moxifloxacin and gatifloxacin in humans. Retina 2006; 26:191-195.

711. Burka JM, Bower KS, Vanroekel RC et al. The effect of fourth-generation fluoroquinolones gatifloxacin and moxifloxacin on epithelial healing following photorefractive keratectomy. Am J Ophthalmol 2005; 140:83-87.

712. Durrie DS, Trattler W. A comparison of therapeutic regimens containing moxifloxacin $0.5 \%$ ophthalmic solution and gatifloxacin $0.3 \%$ ophthalmic solution for surgical prophylaxis in patients undergoing LASIK or LASEK. J Ocul Pharmacol Ther 2005; 21:236-241.

713. Campos M, Ávila M, Wallau A et al. Efficacy and tolerability of a fixed-dose moxifloxacin-dexamethasone formulation for topical prophylaxis in LASIK: a comparative, double-masked clinical trial. Clin Ophthalmol 2008; 2:331338.

714. Freitas LL, Soriano E, Muccioli C et al. Efficacy and tolerability of a combined moxifloxacin/dexamethasone formulation for topical prophylaxis and reduction of inflammation in phacoemulsification: a comparative, double masked clinical trial. Curr Med Res Opin 2007; 23:31233130.

715. Lane SS, Osher RH, Masket S et al. Evaluation of the safety of prophylactic intracameral moxifloxacin in cataract surgery. I Cataract Refract Surg 2008; 34:14511459.

716. Espiritu CR, Caparas VL, Bolinao JG. Safety of prophylactic intracameral moxifloxacin $0.5 \%$ ophthalmic solution in cataract surgery patients. J Cataract Refract Surg 2007; 33:63-68.

717. Olson RJ. Reducing the risk of postoperative endophthalmitis. Surv Ophthalmol 2004; 49(suppl 2):s55-61.

718. Yu-Wai-Man P, Morgan SJ, Hildreth AJ et al. Efficacy of intracameral and subconjunctival cefuroxime in preventing endophthalmitis after cataract surgery. J Cataract Refract Surg 2008; 34:447-451.

719. Vasavada AR, Gajjar D, Raj SM et al. Comparison of 2 moxifloxacin regimens for preoperative prophylaxis: prospective randomized triple-masked trial. Part 1: aqueous concentration of moxifloxacin. J Cataract Refract Surg 2008; 34:1379-1382.

720. Eron LJ. Prevention of infection following orthopedic surgery. Antibiot Chemother 1985; 33:140-164.

721. Gosselin RA, Roberts I, Gillespie WJ. Antibiotics for preventing infection in open limb fractures. Cochrane Database Syst Rev 2004; 1:CD003764.

722. Hauser CJ, Adams CA Jr, Eachempati SR et al. Surgical Infection Society guideline: prophylactic antibiotic use in open fractures: an evidence-based guideline. Surg Infect 2006; 7:379-405.

723. Jaeger M, Maier D, Kern WV et al. Antibiotics in trauma and orthopedic surgery-a primer of evidence-based recommendations. Injury 2006; 37:s74-s80.

724. Luchette FA, Bone LB, Born CT et al. Practice management guidelines for prophylactic antibiotic use in open fractures. www.east.org/tpg/openfrac.pdf (accessed 2008 May 26).

725. Whitehouse JD, Friedman ND, Kirkland KB et al. The impact of surgical-site infections following orthopedic surgery at a community hospital and university hospital: adverse quality of life, excess length of stay and extra cost. Infect Control Hosp Epidemiol 2002; 23:183-189.

726. Boyd RJ, Burke JF, Colton T. A double-blind clinical trial of prophylactic antibiotics in hip fractures. J Bone Joint Surg 1973; 55:1251-1258.

727. Sculco TP. The economic impact of infected joint arthroplasty. Orthopaedics 1995; 18:871-873. 
728. Kurtz SM, Lau E, Schmier J et al. Infection burden for hip and knee arthroplasty in the United States. J Arthroplasty 2008; 23:984-991.

729. Bozic KJ, Ries MD. The impact of infection after total hip arthroplasty on hospital and surgeon resource utilization. J Bone Joint Surg Am 2005; 87:1746-1751.

730. Hebert CK, Williams RE, Levy RS et al. Cost of treating an infected total knee replacement. Clin Orthop Related Res 1996; 331:140-145.

731. Anderson DJ, Kaye KS, Schmader KE et al. Clinical and financial outcomes due to methicillin resistant Staphylococcus aureus surgical site infection: a multi-center matched outcomes study. PLoS One 2009; 4:e8305.

732. Brown EM, Path FR, Pople IK et al. Prevention of postoperative infection in patients undergoing spinal surgery. Spine 2004; 29:938-945.

733. Barker FG II. Efficacy of prophylactic antibiotic therapy in spinal surgery: a meta-analysis. Neurosurgery 2002; 51:391-401.

734. Ericson C, Lidgren L, Lindberg L. Cloxacillin in the prophylaxis of postoperative infections of the hip. I Bone Joint Surg 1973; 55:808-813.

735. Pollard JP, Hughes SP, Scott JE et al. Antibiotic prophylaxis in total hip replacement. Br Med J 1979; 1:707-709.

736. Burnett JW, Gustilo RB, Williams DN et al. Prophylactic antibiotics in hip fractures. J Bone Joint Surg 1980; 62:457462.

737. Tengve B, Kjellander J. Antibiotic prophylaxis in operations on trochanteric femoral fractures. J Bone Joint Surg 1978; 60:97-99.

738. Pavel A, Smith RL, Ballard A et al. Prophylactic antibiotics in clean orthopedic surgery. J Bone Joint Surg 1974; 56:777-782.

739. Boxma H, Broekhuizen $\mathrm{T}$, Patka $\mathrm{P}$ et al. Randomised controlled trial of single-dose antibiotic prophylaxis in surgical treatment of closed fractures: the Dutch Trauma Trial. Lancet 1996; 347:1133-1137.

740. Weinstein MA, McCabe JP, Cammisa FP Jr. Postoperative spinal wound infection: a review of 2,391 consecutive index procedures. J Spinal Disord 2000; 13:422-426.

741. Trampuz A, Zimmerli W. Antimicrobial agents in orthopedic surgery: prophylaxis and treatment. Drugs 2006; 66:1089-1105.

742. Yamaguchi K, Adams RA, Morrey BF. Infection after total elbow arthroplasty. J Bone Joint Surg Am 1998; 80:481-91.

743. Bohsali KI, Wirth MA, Rockwood CA Jr. Complications of total shoulder arthroplasty. J Bone Joint Surg Am 2006; 88:2279-2292.

744. Meehan J, Jamali AA, Nguyen H. Prophylactic antibiotics in hip and knee arthroplasty. J Bone Joint Surg Am 2009; 91:2480-2490.

745. Costerton JW, Stewart PS, Greenberg EP. Bacterial biofilms: a common cause of persistent infections. Science 1999; 284:1318-1322.

746. Costerton JW. Biofilm theory can guide the treatment of device-related orthopaedic infections. Clin Orthop Related Res 2005; 437:7-11.

747. Lewis K. Riddle of biofilm resistance. Antimicrob Agents Chemother 2001; 45:999-1007.

748. Matthews PC, Berendt AR, McNally MA et al. Diagnosis and management of prosthetic joint infection. BMJ 2009; 338:1378-1383.

749. Prokuski L. Prophylactic antibiotics in orthopaedic surgery. I Am Acad Orthop Surg 2008; 16:283-293.
750. Gernaat-Van Der Sluis AJ, Hoogenboom-Verdegaal AM, Edixhoven PJ et al. Prophylactic mupirocin could reduce orthopedic wound infections: 1044 patients treated with mupirocin compared with 1260 historical controls. Acta Orthop Scand 1998; 69: 412-414.

751. Wilcox $\mathrm{MH}, \mathrm{Hall} \mathrm{J}$, Pike $\mathrm{H}$ et al. Use of perioperative mupirocin to prevent methicillin resistant Staphylococcus aureus (MRSA) orthopedic surgical site infections. J Hosp Infect 2003; 54:196-201.

752. Coskun D, Aytac J. Decrease in Staphylococcus aureus surgical-site infection rates after orthopaedic surgery after intranasal mupirocin ointment. J Hosp Infect 2004; 58:90-91.

753. Van Rijen MM, Bonten M, Wenzel RP et al. Intranasal mupirocin for reduction of Staphylococcus aureus infections in surgical patients with nasal carriage: a systematic review. J Antimicrob Chemother 2008; 61:254-261.

754. Rao N, Cannella B, Crossett LS et al. A preoperative decolonization protocol for Staphylococcus aureus prevents orthopaedic infections. Clin Orthop Relat Res 2008; 466: 1343-1348.

755. Kim DH, Spencer M, Davidson SM et al. Institutional prescreening for detection and eradication of methicillinresistant Staphylococcus aureus in patients undergoing elective orthopaedic surgery. I Bone Joint Surg Am 2010; 92:1820-1826.

756. Zgonis T, Jolly GP, Garbalosa JC. The efficacy of prophylactic intravenous antibiotics in elective foot and ankle surgery. J Foot Ankle Surg 2004; 43:97-103.

757. Kurzweil PR. Antibiotic prophylaxis for arthroscopic surgery. Arthroscopy 2006; 22:452-454.

758. Wieck JA, Jackson JK, O'Brien TJ et al. Efficacy of prophylactic antibiotics in arthroscopic surgery. Orthopedics 1997; 20:133-134.

759. Bert JM, Giannini D, Nace L. Antibiotic prophylaxis for arthroscopy of the knee: is it necessary? Arthroscopy 2007; 23:4-6.

760. Babcock HM, Carroll C, Matava M et al. Surgical site infections after arthroscopy: outbreak investigation and case control study. Arthroscopy 2003; 19:172-181.

761. Olix ML, Klug TJ, Coleman CR et al. Prophylactic antibiotics in elective operations on bones, joints, and tendons. Surg Forum 1960; 10:818-819.

762. Tachdjian MO, Compere EL. Postoperative wound infections in orthopedic surgery: evaluation and prophylactic antibiotics. J Int Coll Surg 1957; 28:797-805.

763. Beiner JM, Grauer J, Kwon BK et al. Postoperative wound infections of the spine. Neurosurg Focus 2003; 15:1-5.

764. Labbé AC, Demers AM, Rodrigues R et al. Surgical-site infection following spinal fusion: a case-control study in a children's hospital. I Infect Control Hosp Epidemiol 2003; 24:591-595.

765. Lonstein J, Winter R, Moe J et al. Wound infection with Harrington instrumentation and spine fusion for scoliosis. Clin Orthop Relat Res 1973; 96:222-233.

766. Dimick JB, Lipsett PA, Kostuik JP. Spine update: antimicrobial prophylaxis in spine surgery: basic principles and recent advances. Spine 2000; 25:2544-2548.

767. Kanafani ZA, Dakdouki GK, El-Dbouni O et al. Surgical site infections following spinal surgery at a tertiary care center in Lebanon: incidence, microbiology, and risk factors. Scand J Infect Dis 2006; 38:589-592.

768. O'Toole JE, Eichholz KM, Fessler RG. Surgical site infection rates after minimally invasive spinal surgery. J Neurosurg Spine 2009; 11:471-476. 
769. Hellbusch LC, Helzer-Julin M, Doran SE et al. Single-dose vs multiple-dose antibiotic prophylaxis in instrumented lumbar fusion-a prospective study. Surg Neurol 2008; 70:622-627.

770. Pull Ter Gunne AF, van Laarhoven CJ, Cohen DB. Incidence of surgical site infection following adult spinal deformity surgery: an analysis of patient risk. Eur Spine J 2010; 19:982-988.

771. Wimmer C, Gluch H, Franzreb M et al. Predisposing factors for infection in spine surgery: a survey of 850 spinal procedures. J Spinal Disord 1998; 11:125-128.

772. Friedman ND, Sexton DJ, Connelly SM et al. Risk factors for surgical site infection complicating laminectomy. Infect Control Hosp Epidemiol 2007; 28:1060-1065.

773. Olsen MA, Nepple JJ, Riew D et al. Risk factors for surgical site infection following orthopaedic spinal operations. I Bone Joint Surg Am 2008; 90:62-69.

774. Hollenbeak CS, Lave JR, Zeddies T et al. Factors associated with risk of surgical wound infections. Am J Med Qual 2006; 21(suppl):29S-34S.

775. Pull Ter Gunne AF, Cohen DB. Incidence, prevalance, and analysis of risk factors for surgical site infection following adult spinal surgery. Spine 2009; 34:1422-1428.

776. Fang A, Hu SS, Endres $\mathrm{N}$ et al. Risk factors for infection after spinal surgery. Spine 2005; 30:1460-1465.

777. Patel N, Bagan B, Vadera S et al. Obesity and spine surgery: relations to perioperative complications. I Neurosurg Spine 2007; 6:291-297.

778. Petignat C, Francioli P, Harbarth S et al. Cefuroxime prophylaxis is effective in noninstrumented spine surgery: a double-blind, placebo-controlled study. Spine 2008; 33:1919-1924.

779. Walters R, Moore R, Fraser R. Penetration of cefazolin in human lumbar intervertebral disk. Spine 2006; 31:567-570.

780. Rimoldi RL, Haye W. The use of antibiotics for wound prophylaxis in spinal surgery. Orthop Clin North Am 1996; 27:47-52.

781. American Academy of Orthopaedic Surgeons. Information statement: the use of prophylactic antibiotics in orthopaedic medicine and the emergence of vancomycinresistant bacteria. www.aaos.org/about/papers/advistmt/ 1016.asp (accessed 2008 May 13).

782. Dobzyniak MA, Fischgrund JS, Hankins S et al. Single versus multiple dose antibiotic prophylaxis in lumbar disc surgery. Spine 2003; 28:453-455.

783. Milstone AM, Maragakis LL, Townsend T et al. Timing of preoperative antibiotic prophylaxis: a modifiable risk factor for deep surgical site infections after pediatric spinal fusion. Pediatr Infect Dis J 2008; 27:704-708.

784. Coe JD, Smithe JS, Berven $S$ et al. Complications of spinal fusion for Scheuermann kyphosis: a report of the Scoliosis Research Society Morbidity and Mortality Committee. Spine 2010; 35:99-103.

785. Linam WM, Margolis PA, Staat MA et al. Risk factors associated with surgical site infection after pediatric posterior spinal fusion procedure. Infect Control Hosp Epidemiol 2009; 30:109-116.

786. Fitzgerald RH. Infections of hip prosthesis and artificial joints. Infect Dis Clin North Am 1989; 3:329-338.

787. Southwell-Keely JP, Russo RR, App B et al. Antibiotic prophylaxis in hip fracture surgery: a meta-analysis. Clin Orthop Relat Res 2004; 410:179-184.

788. Gillespie WJ, Walenkamp G. Antibiotic prophylaxis for surgery for proximal femoral and other closed long bone fractures. Cochrane Database Syst Rev 2001; 1: CD000244.

789. Hahnel J, Burdekin H, Anand S. Re-admissions following hip fracture surgery. Ann R Coll Surg Engl 2009; 91:591595.

790. Gulihar A, Nixon M, Jenkins D et al. Clostridium difficile in hip fracture patients: prevention, treatment and associated mortality. Injury 2009; 40:746-751.

791. Cunha BA, Gossling HR, Pasternak HS et al. The penetration characteristics of cefazolin, cephalothin, and cephradine into bone in patients undergoing total hip replacement. I Bone Joint Surg 1977; 59:856-859.

792. Starks I, Ayub G, Walley G et al. Single-dose cefuroxime with gentamicin reduces Clostridium difficile-associated disease in hip-fracture patients. I Hosp Infect 2008; 70: $21-26$.

793. DeFrances CJ, Hall MJ. 2005 National Hospital Discharge Survey. Adv Data 2007; 385:1-19.

794. Glazebrook MA, Arsenault K, Dunbar M. Evidence-based classification of complications in total ankle arthroplasty. Foot Ankle Int 2009; 30:945-949.

795. Gougoulias N, Khanna A, Maffulli N. How successful are current ankle replacements? A systematic review of the literature. Clin Orthop Relat Res 2010; 468:199-208.

796. Blom AW, Brown J, Taylor AH et al. Infection after total knee arthroplasty. J Bone Joint Surg Br 2004; 86:688-691.

797. Kasten MD, Skinner HB. Total elbow arthroplasty. An 18year experience. Clin Orthop Relat Res 1993; 290:177-188.

798. Periti P, Stringa G, Mini E et al. Comparative multicenter trial of teicoplanin versus cefazolin for antimicrobial prophylaxis in prosthetic joint implant surgery. Eur J Clin Microbiol Infect Dis 1999; 18:113-119.

799. Minnema B, Vearncombe M, Augustin A et al. Risk factors for surgical-site infection following primary total knee arthroplasty. Infect Control Hosp Epidemiol 2004; 25:477480.

800. Blom AW, Taylor AH, Pattison G et al. Infection after total hip arthroplasty. J Bone Joint Surg Br 2003; 85:956-959.

801. AlBuhairan B, Hind D, Hutchinson A. Antibiotic prophylaxis for wound infections in total joint arthroplasty. I Bone Joint Surg Br 2008; 90:915-919.

802. Fish DN, Hoffman HM, Danziger LH. Antibiotic impregnated cement use in U.S. hospitals. Am J Hosp Pharm 1992; 49:2469-2474.

803. Malik MH, Gambhir AK, Bale L et al. Primary total hip replacement: a comparison of a nationally agreed guide to practice and current surgical technique as determined by the North West Regional Arthroplasty Registry. Ann R Coll Surg Engl 2004; 86:113-118.

804. Bourne RB. Prophylactic use of antibiotic bone cement: an emerging standard-in the affirmative. I Arthroplasty 2004; 19(suppl 1):69-72.

805. Engesaeter LB, Lie SA, Espehaug B et al. Antibiotic prophylaxis in total hip arthroplasty: effects of antibiotic prophylaxis systemically and in bone cement on the revision rate of 22,170 primary hip replacements followed 0 to 14 years in the Norwegian Arthroplasty Register. Acta Orthop Scand 2003; 74:644-651.

806. Espehaug B, Engesaeter LB, Vollset SE et al. Antibiotic prophylaxis in total hip arthroplasty: review of 10,905 primary cemented total hip replacements reported to the Norwegian Arthroplasty Register, 1987 to 1995. J Bone Joint Surg Br 1997; 79:590-595. 
807. Jiranek WA, Hanssen AD, Greenwald AS. Antibioticloaded bone cement for infection prophylaxis in total joint replacement. J Bone Joint Surg 2006; 88:2487-2500.

808. McQueen MM, Hughes SP, May P et al. Cefuroxime in total joint arthroplasty. Intravenous or in bone cement. I Arthroplasty 1990; 5:169-172.

809. Josefsson G, Kolmert L. Prophylaxis with systematic antibiotics versus gentamicin bone cement in total hip arthroplasty. A ten-year survey of 1,688 hips. Clin Orthop Relat Res 1993; 292:210-214.

810. Hanssen AD, Osmon DR. The use of prophylactic antimicrobial agents during and after hip arthroplasty. Clin Orthop Relat Res 1999; 369:124-138.

811. Jiranek W. Antibiotic-loaded cement in total hip replacement: current indications, efficacy, and complications. Orthopedics 2005; 28(suppl):S873-S877.

812. Diefenbeck M, Mückley T, Hofman GO. Prophylaxis and treatment of implant-related infections by local application of antibiotics. Injury 2006; 37(suppl 2):S95-S104.

813. Hanssen AD. Prophylactic use of antibiotic bone cement: an emerging standard-in opposition. I Arthroplasty 2004; 19(suppl 1):73-77.

814. Block JE, Stubbs HA. Reducing the risk of deep wound infection in primary joint arthroplasty with antibiotic bone cement. Orthopedics 2005; 28:1334-1345.

815. Wininger DA, Fass RJ. Antibiotic-impregnated cement and beads for orthopedic infections. Antimicrob Chemother 1996; 40:2675-2679.

816. Nelson CL, Green TG, Porter RA et al. One day versus seven days of preventive antibiotic therapy in orthopedic surgery. Clin Orthop Relat Res 1983; 176:258-263.

817. Matsumoto T, Kiyota H, Matsukawa M et al. Japanese guidelines for prevention of perioperative infections in urological field. Int J Urol 2007; 14:890-909.

818. Wolf JS Jr, Bennett CJ, Dmochowski RR et al. Best practice policy statement on urologic surgery antimicrobial prophylaxis. J Urol 2008; 179:1379-1390.

819. Kapoor DA, Klimberg IW, Malek GH et al. Single-dose oral ciprofloxacin versus placebo for prophylaxis during transrectal prostate biopsy. Urology 1998; 52:552-558.

820. Latthe PM, Foon R, Toozs-Hobson P. Prophylactic antibiotics in urodynamics: a systematic review of effectiveness and safety. Neurourol Urodyn 2008; 27:167-173.

821. Kartal ED, Yenilmez A, Kiremitci A et al. Effectiveness of ciprofloxacin prophylaxis in preventing bacteriuria caused by urodynamic study: a blind, randomized study of 192 patients. Urology 2006; 67:1149-1153.

822. Wagenlehner FM, Wagenlehner C, Schinzel S et al. Prospective, randomized, multicentric, open, comparative study on the efficacy of a prophylactic single dose of $500 \mathrm{mg}$ levofloxacin versus $1920 \mathrm{mg}$ trimethoprim/sulfamethoxazole versus a control group in patients undergoing TUR of the prostate. Eur Urol 2005; 47:549-556.

823. Takeyama K, Takahashi S, Maeda T et al. Comparison of 1-day, 2-day, and 3-day administration of antimicrobial prophylaxis in radical prostatectomy. I Infect Chemother 2007; 13:320-323.

824. Briffaux R, Coloby P, Bruyere F et al. One preoperative dose randomized against 3-day antibiotic prophylaxis for transrectal ultrasonography-guided prostate biopsy. BJU Int 2008; 103:1069-1073.

825. Bootsma AM, Pes MP, Geerlings SE et al. Antibiotic prophylaxis in urologic procedures: a systematic review. Eur Urol 2008; 54:1270-1286.
826. Grabe M, Bishop MC, Bjerklund-Johansen TE et al. Guidelines on urological infections. www.uroweb.org (accessed 2010 Mar 18).

827. Yamamoto S, Kanamaru S, Kunishima Y et al. Perioperative antimicrobial prophylaxis in urology: a multicenter prospective study. J Chemother 2005; 17:189-197.

828. Hamasuna R, Betsunoh $\mathrm{H}$, Sueyoshi $\mathrm{T}$ et al. Bacteria of preoperative urinary tract infections contaminate the surgical fields and develop surgical site infections in urologic operations. Int J Urol 2004; 11:941-947.

829. Richter S, Lang R, Zur F et al. Infected urine as a risk factor for postprostatectomy wound infection. Infect Control Hosp Epidemiol 1991; 12:147-149.

830. Carson CC. Diagnosis, treatment and prevention of penile prosthesis infection. Int J Impot Res 2003; 15(suppl 5): S139-S146.

831. Schaeffer AJ, Montorsi F, Scattoni V et al. Comparison of a 3-day with a 1-day regimen of an extended-release formulation of ciprofloxacin as antimicrobial prophylaxis for patients undergoing transrectal needle biopsy of the prostate. BJU Int 2007; 100:51-57.

832. Hara N, Kitamura $Y$, Saito $T$ et al. Perioperative antibiotics in radical cystectomy with ileal conduit urinary diversion: efficacy and risk of antimicrobial prophylaxis on the operation day alone. Int J Urol 2008; 15:511-515.

833. Meir DB, Livne PM. Is prophylactic antimicrobial treatment necessary after hypospadias repair? J Urol 2004; 171:2621-2622.

834. Doğan HS, Şahin A, Çetinkaya Y et al. Antibiotic prophylaxis in percutaneous nephrolithotomy: prospective study in 81 patients. J Endourol 2002; 16:649-653.

835. Cox CE. Comparison of intravenous ciprofloxacin and intravenous cefotaxime for antimicrobial prophylaxis in transurethral surgery. Am J Med 1989; 87(suppl 5A):252S$254 \mathrm{~S}$.

836. Cam K, Kayikci A, Akman Y et al. Prospective assessment of the efficacy of single dose versus traditional 3-day antimicrobial prophylaxis in 12-core transrectal prostate biopsy. Int J Urol 2008; 18:997-1001.

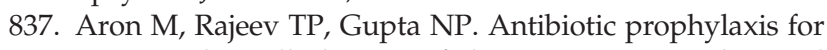
transrectal needle biopsy of the prostate: a randomized controlled study. BJU Int 2000; 85:682-685.

838. Isen K, Küpeli B, Sinik Z et al. Antibiotic prophylaxis for transrectal biopsy of the prostate: a prospective randomized study of the prophylactic use of single dose oral fluoroquinolone versus trimethoprim-sulfamethoxazole. Int Urol Nephrol 1999; 31:491-495.

839. Johnson MI, Merrilees D, Robson WA et al. Oral ciprofloxacin or trimethoprim reduces bacteriuria after flexible cystoscopy. BJU Int 2007; 100:826-829.

840. Yokoyama M, Fujii Y, Yoshida S et al. Discarding antimicrobial prophylaxis for transurethral resection of bladder tumor: a feasibility study. Int J Urol 2009; 16: 61-63.

841. Takeyama K, Matsukawa M, Kunishima Y et al. Incidence of and risk factors for surgical site infections in patients with radical cystectomy with urinary diversion. I Infect Chemother 2005; 11:177-181.

842. Gomelsky A, Dmochowski RR. Antibiotic prophylaxis in urologic prosthetic surgery. Curr Pharm Des 2003; 9: 989-996.

843. Hoffelt SC, Wallner K, Merrick G. Epididymitis after prostate brachytherapy. Urology 2004; 63:293-296. 
844. Ferguson $\mathrm{KH}, \mathrm{McNeil} \mathrm{JJ}$, Morey AF. Mechanical and antibiotic bowel preparation for urinary diversion surgery. I Urol 2002; 167:2352-2356.

845. Pearle MS, Roehrborn CG. Antimicrobial prophylaxis prior to shock wave lithotripsy in patients with sterile urine before treatment: a meta-analysis and costeffectiveness analysis. Urology 1997; 49:679-686.

846. Berry A, Barratt A. Prophylactic antibiotic use in transurethral prostatic resection: a meta analysis. J Urol 2002; 167:571-577.

847. Qiang W, Jianchen W, MacDonald R et al. Antibiotic prophylaxis for transurethral prostatic resection in men with preoperative urine containing less than 100,000 bacteria per ml: a systematic review. J Urol 2005; 173: 1175-1181.

848. Knopf HJ, Graff H, Schulze H. Perioperative antibiotic prophylaxis in ureteroscopic stone removal. Eur Urol 2003; 44:115-118.

849. Brewster SF, MacGowan AP, Gingell JC. Antimicrobial prophylaxis for transrectal prostatic biopsy: a prospective, randomized trial of cefuroxime versus piperacillin/tazobactam. Br J Urol 1995; 76:351-354.

850. DeBessonet DA, Merlin AS. Antibiotic prophylaxis in elective genitourinary tract surgery: a comparison of singledose preoperative cefotaxime and multiple-dose cefoxitin. J Antimicrob Chemother 1984; 14(suppl B):271-275.

851. Christiano AP, Hollowell CM, Kim H et al. Double-blind randomized comparison of single-dose ciprofloxacin versus intravenous cefazolin in patients undergoing outpatient endourologic surgery. Urology 2000; 55:182-185.

852. Bhatia NN, Karram MM, Bergman A. Role of antibiotic prophylaxis in retropubic surgery for stress urinary incontinence. Obstet Gynecol 1989; 74:637-639.

853. Terai A, Ichioka $\mathrm{K}$, Kohei $\mathrm{N}$ et al. Antibiotic prophylaxis in radical prostatectomy: 1-day versus 4 -day treatments. Int J Urol 2006; 13:1488-1493.

854. Gombert ME, DuBouchet L, Aulicino TM et al. Brief report: intravenous ciprofloxacin versus cefotaxime prophylaxis during transurethral surgery. Am J Med 1989; 87(suppl 5A): 250S-251S.

855. Klimberg IW, Malek GH, Cox CE. Single-dose oral ciprofloxacin compared with cefotaxime and placebo for prophylaxis during transurethral surgery. I Antimicrob Chemother 1999; 43(suppl A):77-84.

856. Gibbons RP, Stark RA, Gorrea RJ et al. The prophylactic use - or misuse - of antibiotics in transurethral prostatectomy. J Urol 1978; 119:381-383.

857. Ramsey E, Sheth NK. Antibiotic prophylaxis in patients undergoing prostatectomy. Urology 1983; 21:376-378.

858. Cormio L, Berardi B, Callea A et al. Antimicrobial prophylaxis for transrectal prostatic biopsy: a prospective study of ciprofloxacin vs. piperacillin/tazobactam. BJU Int 2002; 90:700-702.

859. Sakura M, Kawakami S, Yoshida S et al. Prospective comparative study of single dose versus 3-day administration of antimicrobial prophylaxis in minimum incision endoscopic radical prostatectomy. Int J Urol 2008; 15: 328-331.

860. Hills NH, Bultitude MI, Eykyn S. Co-trimoxazole in prevention of bacteriuria after prostatectomy. Br Med J 1976; 2:498-499.

861. Matthew AD, Gonzales R, Jeffords D et al. Prevention of bacteriuria after transurethral prostatectomy with nitrofurantoin macrocrystals. I Urol 1978; 120:442-443.
862. Hammarsten J, Lindqvist K. Norfloxacin as prophylaxis against urethral strictures following transurethral resection of the prostate: an open, prospective, randomized study. J Urol 1993; 150:1722-1724.

863. Siracusano S, Knez R, Tiberio A et al. The usefulness of antibiotic prophylaxis in invasive urodynamics in postmenopausal female subjects. Int Urogynecol J 2008; 19: 939-942.

864. Patel U, Kirby R. Infections after prostate biopsy and antibiotic resistance. BJU Int 2008; 101:1201-1204.

865. Hall JC, Christiansen KJ, England P et al. Antibiotic prophylaxis for patients undergoing transurethral resection of the prostate. Urology 1996; 47:852-856.

866. Liu GG, Nguyen T, Nichol MB. An economic analysis of antimicrobial prophylaxis against urinary tract infection in patients undergoing transurethral resection of the prostate. Clin Ther 1999; 21:1589-1603.

867. Burnakis TG. Surgical antimicrobial prophylaxis: principles and guidelines. Pharmacotherapy 1984; 4:248-271.

868. Homer-Vanniasinkam S. Surgical site and vascular infections: treatment and prophylaxis. Int J Infect Dis 2007; 11:S17-S22.

869. Zibari GB, Gadallah MF, Landreneau $M$ et al. Preoperative vancomycin prophylaxis decreases incidence of postoperative hemodialysis vascular access infections. Am J Kidney Dis 1997; 30:343-348.

870. Naylor AR, Payne D, London NJ et al. Prosthetic patch infection after carotid endarterectomy. Eur J Vasc Endovasc Surg 2002; 23:11-16.

871. Richet HM, Chidiac C, Prat A et al. Analysis of risk factors for surgical wound infections following vascular surgery. Am J Med 1991; 91:171S-172S.

872. Ross CB, Wheeler WG II, Jones MJ et al. Ceftriaxone versus cefazolin in peripheral arterial operations: a randomized, prospective trial. South Med J 1997; 90:16-22.

873. Ryan JM, Ryan BM, Smith TP. Antibiotic prophylaxis in interventional radiology. I Vasc Interv Radiol 2004; 15: 547-556.

874. McDermott VG, Schuster MG, Smith TP. Antibiotic prophylaxis in vascular and interventional radiology. Am J Roentgenol 1997; 169:31-38.

875. Beddy P, Ryan JM. Antibiotic prophylaxis in interventional radiology - anything new? Tech Vasc Interv Radiol 2006; 9:69-76.

876. Malek AM, Higashida RT, Reilly LM et al. Subclavian arteritis and pseudoaneurysm formation secondary to stent infection. Cardiovasc Interv Radiol 2000; 23:57-60.

877. Venkatesan AM, Kundu S, Sacks D et al. Practice guideline for adult antibiotic prophylaxis during vascular and interventional radiology procedures. I Vasc Interv Radiol 2010; 21:1611-1630.

878. Thompson M. An audit demonstrating a reduction in MRSA infection in a specialized vascular unit resulting from a change in infection control protocol. Eur J Vasc Endovasc Surg 2006; 31:609-615.

879. Morange-Saussier V, Giraudeau B, van der Mee N et al. Nasal carriage of methicillin-resistant Staphylococcus aureus in vascular surgery. Ann Vasc Surg 2006; 20:767-772.

880. Taylor MD, Napolitano LM. Methicillin-resistant Staphylococcus aureus infections in vascular surgery: increasing prevalence. Surg Infect 2004; 5:180-187.

881. Grimble SA, Magee TR, Galland RB. Methicillin resistant Staphylococcus aureus in patients undergoing major amputation. Eur J Vasc Endovasc Surg 2001; 22:215-218. 
882. Nasim A, Thompson MM, Naylor AR et al. The impact of MRSA on vascular surgery. Eur J Vasc Endovasc Surg 2001; 22:211-214.

883. Cowie SE, Ma I, Lee SK et al. Nosocomial MRSA infection in vascular surgery patients: impact on patient outcome. Vasc Endovasc Surg 2005; 39:327-334.

884. Fawley WN, Parnell P, Hall J et al. Surveillance for mupirocin resistance following introduction of routine perioperative prophylaxis with nasal mupirocin. J Hosp Infect 2006; 62:327-332.

885. Kaiser A, Clayton KR, Mulherin JL et al. Antibiotic prophylaxis in vascular surgery. Ann Surg 1978; 188:283-289.

886. Edwards WH, Kaiser AB, Kernodle DS et al. Cefuroxime versus cefazolin as prophylaxis in vascular surgery. $\underline{\mathrm{J} \text { Vasc }}$ Surg 1992; 15:35-42.

887. Edwards WH Jr, Kaiser AB, Tapper S et al. Cefamandole versus cefazolin in vascular surgical wound infection prophylaxis: cost-effectiveness and risk factors. I Vasc Surg 1993; 18:470-475.

888. Hasselgren PO, Ivarson L, Risberg B et al. Effects of prophylactic antibiotics in vascular surgery. A prospective, randomized, double-blind study. Ann Surg 1984; 200: 86-92.

889. Risberg B, Drott C, Dalman P et al. Oral ciprofloxacin versus intravenous cefuroxime as prophylaxis against postoperative infection in vascular surgery: a randomized double-blind, prospective multicentre study. Eur J Endovasc Surg 1995; 10:346-351.

890. Stewart AH, Eyers PS, Earnshaw JJ. Prevention of infection in peripheral arterial reconstruction: a systematic review and meta-analysis. J Vasc Surg 2007; 46:148-155.

891. Murray BE. Problems and dilemmas of antimicrobial resistance. Pharmacotherapy 1992; 12:86-93.

892. Earnshaw JJ, Slack RC, Hopkinson BR et al. Risk factors in vascular surgical sepsis. Ann R Coll Surg Engl 1988; 70:139-143.

893. Hall JC, Christiansen KJ, Goodman M et al. Duration of antimicrobial prophylaxis in vascular surgery. Am J Surg 1998; 175:87-90.

894. Fishman JA. Infection in solid-organ transplant recipients. N Engl J Med 2007; 357:2601-2614.

895. Fischer SA. Infections in the transplant recipient. Med Health R I 2002; 85:125-127.

896. Soave R. Prophylaxis strategies for solid-organ transplantation. Clin Infect Dis 2001; 33(suppl 1):S26-S31.

897. Keough WL, Michaels MG. Infectious complications in pediatric solid organ transplantation. Pediatr Clin North Am 2003; 50:1451-1469.

898. United Network for Organ Sharing. Organ Procurement and Transplantation Network data (as of September 5, 2008). http://optn.transplant.hrsa.gov/data/ (accessed 2012 Dec 9).

899. Cai J. Thoracic transplantation in the United States: an analysis of UNOS registry data. Clin Transplant 2006; $41-56$.

900. Fong I, Baker C, McKee D. The value of prophylactic antibiotics in aorta-coronary bypass operations. J Thorac Cardiovasc Surg 1979; 78:908-913.

901. Penketh A, Wansbrough-Jones M, Wright E et al. Antibiotic prophylaxis for coronary artery bypass graft surgery. Lancet 1985; 1:1500.

902. Muñoz P, Menasalvas A, Bernaldo de Quirós JC et al. Postsurgical mediastinitis: a case-control study. Clin Infect Dis $1997 ; 25: 1060-1064$.
903. Filsoufi F, Rahmanian PB, Castillo JG et al. Incidence, treatment strategies and outcome of deep sternal wound infection after orthotopic heart transplantation. J Heart Lung Transplant 2007; 26:1084-1090.

904. Abid Q, Nkere UU, Hasan A et al. Mediastinitis in heart and lung transplantation: 15 years experience. Ann Thorac Surg 2003; 75:1565-1571.

905. Carrier M, Perrault LP, Pellerin M et al. Sternal wound infection after heart transplantation: incidence and results with aggressive surgical treatment. Ann Thorac Surg 2001; 72:719-724.

906. Ramos A, Asensio A, Muñoz E et al. Incisional surgical infection in heart transplantation. Transpl Infect Dis 2008; 10:298-302.

907. Sénéchal M, LePrince $P$, Tezenas du Montcel $S$ et al. Bacterial mediastinitis after heart transplantation: clinical presentation, risk factors and treatment. J Heart Lung Transplant 2004; 23:165-170.

908. Mattner F, Fischer S, Weissbrodt $\mathrm{H}$ et al. Post-operative nosocomial infections after lung and heart transplantation. J Heart Lung Transplant 2007; 26:241-249.

909. Van De Beek D, Kremer WK, Del Pozo JL et al. Effect of infectious diseases on outcome after heart transplant. Mayo Clin Proc 2008; 83:304-308.

910. Keay S. Cardiac transplantation: pre-transplant infectious diseases evaluation and post-transplant prophylaxis. Curr Infect Dis Rep 2002; 4:285-292.

911. Kaiser AB. Use of antibiotics in cardiac and thoracic surgery. In: Sabiston DC Jr, Spencer FC, eds. Surgery of the chest. 6th ed. Philadelphia: W. B. Saunders; 1995:98-116.

912. Khaghani A, Martin M, Fitzgerald M et al. Cefotaxime and flucloxacillin as antibiotic prophylaxis in cardiac transplantation. Drugs 1988; 35(suppl 2):124-126.

913. Montoya JG, Giraldo LF, Efron B et al. Infectious complications among 620 consecutive heart transplant patients at Stanford University Medical Center. Clin Infect Dis 2001; 33:629-640.

914. Petri WA Jr. Infections in heart transplant recipients. Clin Infect Dis 1994; 18:141-148.

915. Trulock EP. Lung transplantation. Am J Respir Crit Care Med 1997; 155:789-818.

916. U.S. Department of Health and Human Services. Organ Procurement and Transplantation Network/Scientific Registry of Transplant Recipients annual report. Table 12.4. Transplant recipient characteristics, 1999 to 2008. Recipients of deceased donor lungs. 2009 May 4. http://optn .transplant.hrsa.gov/ar2009/1204_rec-dgn_lu.htm (accessed 2011 Mar 17).

917. Hosenpud JD, Novick RJ, Bennett LE et al. The registry of the International Society for Heart and Lung Transplantation: thirteenth official report. J Heart Lung Transplant 1996; 15:655-674.

918. Davis RD Jr, Pasque MK. Pulmonary transplantation. Ann Surg 1995; 221:14-28.

919. Kotloff RM, Zuckerman JB. Lung transplantation for cystic fibrosis. Special considerations. Chest 1996; 109:787-798.

920. Campos S, Caramori M, Teixeira R et al. Bacterial and fungal pneumonia after lung transplantation. Transplant Proc 2008; 40:822-824.

921. Krishnam MS, Suh RD, Tomasian A et al. Postoperative complications of lung transplantation: radiologic findings along a time continuum. Radiographics 2007; 27:957-974.

922. Helmi M, Love RB, Welter D et al. Aspergillus infection in lung transplant recipients with cystic fibrosis: risk factors 
and outcomes comparison to other types of transplant recipients. Chest 2003; 123:800-808.

923. Russo MJ, Iribarne A, Hong KN et al. High lung allocation score is associated with increased morbidity and mortality following transplantation. Chest 2010; 137:651-657.

924. Dowling RD, Zenati M, Yousem S et al. Donor-transmitted pneumonia in experimental lung allografts. I Thorac Cardiovasc Surg 1992; 103:767-772.

925. Low DE, Kaiser LR, Haydock DA et al. The donor lung: infectious and pathologic factors affecting outcome in lung transplantation. I Thorac Cardiovasc Surg 1993; 106: 614-621.

926. Steinbach S, Sun L, Jiang RZ et al. Transmissibility of Pseudomonas cepacia infection in clinic patients and lungtransplant recipients with cystic fibrosis. $\mathrm{N}$ Engl J Med 1994; 331:981-987.

927. Dauber JH, Paradis IL, Dummer JS. Infectious complications in pulmonary allograft recipients. Clin Chest Med 1990; 11:291-308.

928. Deusch E, End A, Grimm M et al. Early bacterial infections in lung transplant recipients. Chest 1993; 104:1412-1416.

929. Paradis IL, Williams P. Infection after lung transplantation. Semin Respir Infect 1993; 8:207-215.

930. Husain S, Zaldonis D, Kusne $S$ et al. Variation in antifungal prophylaxis strategies in lung transplantation. Transpl Infect Dis 2006; 8:213-218.

931. Noyes BE, Kurland G, Orenstein DM. Lung and heartlung transplantation in children. Pediatr Pulmonol 1997; 23:39-48.

932. Moreno R, Berenguer M. Post-liver transplantation medical complications. Ann Hepatol 2006; 5:77-85.

933. Muiesan P, Vergani D, Mieli-Vergani G. Liver transplantation in children. J Hepatol 2007; 46:340-348.

934. United Network for Organ Sharing. Organ Procurement and Transplantation Network: data. http://optn. transplant.hrsa.gov/data/. Based on OPTN data as of September 26, 2008.

935. García Prado ME, Matia EC, Ciuro FP et al. Surgical site infection in liver transplant recipients: impact of the type of perioperative prophylaxis. Transplantation 2008; 85: 1849-1854.

936. Kuo PC, Bartlett ST, Lim JW et al. Selective bowel decontamination in hospitalized patients awaiting liver transplantation. Am J Surg 1997; 174:745-749.

937. Kim YJ, Kim SI, Wie SH et al. Infectious complications in living-donor liver transplant recipients: a 9-year singlecenter experience. Transplant Infect Dis 2008; 10:316-324.

938. Hollenbeak CS, Alfrey EJ, Souba WW. The effect of surgical site infections on outcomes and resource utilization after liver transplantation. Surgery 2001; 130:388-395.

939. Kibbler CC. Infections in liver transplantation: risk factors and strategies for prevention. I Hosp Infect 1995; 30(suppl):209-217.

940. Wade JJ, Rolando N, Hayllar K et al. Bacterial and fungal infections after liver transplantation: an analysis of 284 patients. Hepatology 1995; 21:1328-1336.

941. Shepherd RW, Turmelle Y, Nadler M et al. Risk factors for rejection and infection in pediatric liver transplantation. Am J Transplant 2008; 8:396-403.

942. Hollenbeak CS, Alfrey EJ, Sheridan K et al. Surgical site infections following pediatric liver transplantation: risks and costs. Transpl Infect Dis 2003; 5:72-78.

943. Arnow PM, Carandang GC, Zabner $\mathrm{R}$ et al. Randomized controlled trial of selective bowel decontamination for prevention of infections following liver transplantation. Clin Infect Dis 1996; 22:997-1003.

944. Colonna JO II, Drew WJ, Brill JE et al. Infectious complications in liver transplantation. Arch Surg 1988; 123:360-364.

945. George DL, Arnow PM, Fox AS et al. Bacterial infection as a complication of liver transplantation: epidemiology and risk factors. Rev Infect Dis 1991; 13:387-396.

946. Uemoto S, Tanaka K, Fujita S et al. Infectious complications in living related liver transplantation. $\underline{\text { J Pediatr Surg }}$ 1994; 29:514-517.

947. Kusne S, Dummer JS, Singh N et al. Infections after liver transplantation. An analysis of 101 consecutive cases. Medicine 1988; 67:132-143.

948. Singh N, Paterson DL, Gayowski T et al. Predicting bacteremia and bacteremic mortality in liver transplant recipients. Liver Transpl 2000; 6:54-61.

949. Hjortrup A, Rasmussen A, Hansen BA et al. Early bacterial and fungal infections in liver transplantation after oral selective bowel decontamination. Transplant Proc 1997; 29:3106-3110.

950. Villacian JS, Paya CV. Prevention of infections in solid organ transplant recipients. Transpl Infect Dis 1999; 1:50-64.

951. Asensio A, Ramos A, Cuervas-Mons V et al. Effect of antibiotic prophylaxis on the risk of surgical site infection in orthotopic liver transplant. Liver Transpl 2008; 14:799-805.

952. Arnow PM, Zachary KC, Thistlethwaite JR et al. Pathogenesis of early operative site infections after orthotopic liver transplantation. Transplantation 1998; 65:1500-1503.

953. Mattner F, Kola A, Fischer S et al. Impact of bacterial and fungal donor organ contamination in lung, heart-lung, heart and liver transplantation. Infection 2008; 36:207-212.

954. Barkholt LM, Andersson J, Ericzon BG et al. Stool cultures obtained before liver transplantation are useful for choice of perioperative antibiotic prophylaxis. Transplant Int 1997; 10:432-438.

955. Hellinger WC, Yao JD, Alvarez S et al. A randomized, prospective, double-blinded evaluation of selective bowel decontamination in liver transplantation. Transplantation 2002; 73:1904-1909.

956. Zwaveling JH, Maring JK, Klompmaker IJ et al. Selective decontamination of the digestive tract to prevent postoperative infection: a randomized placebo-controlled trial in liver transplant patients. Crit Care Med 2002; 30:12041209.

957. Hashimoto M, Sugawara Y, Tamura S et al. Impact of new methicillin-resistant Staphylococcus aureus carriage postoperatively after living donor liver transplantation. Transplant Proc 2007; 39:3271-3275.

958. Hashimoto M, Sugawara Y, Tamura S et al. Bloodstream infection after living donor liver transplantation. Scand J Infect Dis 2008; 40:509-516.

959. Hashimoto M, Sugawara Y, Tamura S et al. Pseudomonas aeruginosa infection after living-donor liver transplantation in adults. Transpl Infect Dis 2009; 11:11-19.

960. Bert F, Galdbart JO, Zarrouk V et al. Association between nasal carriage of Staphylococcus aureus and infection in liver transplant recipients. Clin Infect Dis 2000; 31:12951299.

961. Bert F, Bellier C, Lassel L et al. Risk factors for Staphylococcus aureus infection in liver transplant recipients. Liver Transpl 2005; 11:1093-1099.

962. Chang FY, Singh N, Gayowski T et al. Staphylococcus aureus nasal colonization and association with infections in 
liver transplant recipients. Transplantation 1998; 65:11691172.

963. Mehrabi A, Fonouni H, Wente $\mathrm{M}$ et al. Wound complications following kidney and liver transplantation. Clin Transplant 2006; 20(suppl 17):97-110.

964. Kawecki D, Chmura A, Pacholczyk M et al. Surgical site infections in liver recipients in the early posttransplantation period: etiological agents and susceptibility profiles. Transplant Proc 2007; 39:2800-2806.

965. Bedini A, Codeluppi M, Cocchi S et al. Gram-positive bloodstream infections in liver transplant recipients: incidence, risk factors, and impact on survival. Transplant Proc 2007; 39:1947-1949.

966. Dar FS, Faraj W, Zaman MB et al. Outcome of liver transplantation in hereditary hemochromatosis. Transplant Int 2009; 22:717-724.

967. Arnow PM, Furmaga K, Flaherty JP et al. Microbiological efficacy and pharmacokinetics of prophylactic antibiotics in liver transplant patients. Antimicrob Agents Chemother 1992; 36:2125-2130.

968. Gorensek MJ, Carey WD, Washington JA II et al. Selective bowel decontamination with quinolones and nystatin reduces gram-negative and fungal infections in orthotopic liver transplant recipients. Cleve Clin J Med 1993; 60:139144.

969. Piselli P, Zanfi C, Corazza V et al. Incidence and timing of infections after liver transplant in Italy. Transplant Proc 2007; 39:1950-1952.

970. Desai D, Desai N, Nightingale P et al. Carriage of methicillin-resistant Staphylococcus aureus is associated with an increased risk of infection after liver transplantation. Liver Transpl 2003; 9:754-759.

971. Rayes N, Seehofer D, Theruvath T et al. Supply of pre- and probiotics reduces bacterial infection rates after liver transplantation - a randomized, double-blind trial. Am J Transplant 2005; 5:125-130.

972. Reid GE, Grim SA, Aldeza CA et al. Rapid development of Acinetobacter baumannii resistance to tigecycline. Pharmacotherapy 2007; 27:1198-1201.

973. Chen H, Zhang Y, Chen YG et al. Sepsis resulting from Enterobacter aerogenes resistant to carbapenems after liver transplantation. Hepatobiliary Pancreat Dis Int 2009; 8: 320-322.

974. Chen YG, Zhang Y, Yu YS et al. In vivo development of carbapenem resistance in clinical isolates of Enterobacter aerogenes producing multiple $\beta$-lactamases. Int J Antimicrob Agents 2008; 32:302-307.

975. Bennett JW, Herrera ML, Lewis JS II et al. KPC-2producing Enterobacter cloacae and Pseudomonas putida coinfection in a liver transplant recipient. Antimicrob Agents Chemother 2009; 53:292-294.

976. Carignan A, Allard C, Pépin J et al. Risk of Clostridium difficile infection after perioperative antibacterial prophylaxis before and during an outbreak of infection due to a hypervirulent strain. Clin Infect Dis 2008; 46:1838-1846.

977. Stelzmueller I, Goegele $\mathrm{H}$, Biebl $\mathrm{M}$ et al. Clostridium difficile in solid organ transplantation-a single-center experience. Dig Dis Sci 2007; 52:3231-3236.

978. Hashimoto M, Sugawara Y, Tamura S et al. Clostridium difficile-associated diarrhea after living donor liver transplantation. World J Gastroenterol 2007; 13:2072-2076.

979. Bion JF, Badger I, Crosby HA et al. Selective decontamination of the digestive tract reduces gram-negative pulmonary colonization but not systemic endotoxemia in patients undergoing elective liver transplantation. Crit Care Med 1994; 22:40-49.

980. Rayes N, Seehofer D, Hansen S et al. Early enteral supply of lactobacillus and fiber versus selective bowel decontamination: a controlled trial in liver transplant recipients. Transplantation 2002; 74:123-128.

981. González-Segura C, Pascual M, Garcia Huete L et al. Donors with positive blood culture: could they transmit infections to the recipients? Transplant Proc 2005; 37:36643666.

982. Lumbreras C, Cuervas-Mons V, Jara P et al. Randomized trial of fluconazole versus nystatin for the prophylaxis of Candida infection following liver transplantation. J Infect Dis 1996; 174:583-588.

983. Winston DJ, Pakrasi A, Busuttil RW. Prophylactic fluconazole in liver transplant recipients. A randomized, double-blind, placebo-controlled trial. Ann Intern Med 1999; 131:729-737.

984. Sharpe MD, Ghent C, Grant D et al. Efficacy and safety of itraconazole prophylaxis for fungal infections after orthotopic liver transplantation: a prospective, randomized, double-blind study. Transplantation 2003; 76:977-983.

985. Castroagudin JF, Ponton C, Bustamante $\mathrm{M}$ et al. Prospective interventional study to evaluate the efficacy and safety of liposomal amphotericin B as prophylaxis of fungal infections in high-risk liver transplant recipients. Transplant Proc 2005; 37:3965-3967.

986. Lorf T, Braun F, Ruchel R et al. Systemic mycoses during prophylactical use of liposomal amphotericin B (Ambisome) after liver transplantation. Mycoses 1999; 42:47-53.

987. Tollemar J, Hockerstedt K, Ericzon BG et al. Liposomal amphotericin B prevents invasive fungal infections in liver transplant recipients. A randomized, placebo-controlled study. Transplantation 1995; 59:45-50.

988. Fortun J, Martin-Davila P, Montejo M et al. Prophylaxis with caspofungin for invasive fungal infections in highrisk liver transplant recipients. Transplantation 2009; 87:424-435.

989. Cruciani M, Mengoli C, Malena M et al. Antifungal prophylaxis in liver transplant patients: a systematic review and meta-analysis. Liver Transpl 2006; 12:850-858.

990. Weisner RH, Hermans PE, Rakela J et al. Selective bowel decontamination to decrease gram-negative aerobic bacterial and candidal colonization and prevent infection after orthotopic liver transplantation. Transplantation 1988; 45:570-574.

991. Safdar N, Said A, Lucey MR. The role of selective digestive decontamination for reducing infection in patients undergoing liver transplantation: a systematic review and meta-analysis. Liver Transpl 2004; 10:817-827.

992. Wiesmayr S, Stelzmueller I, Mark W et al. Experience with the use of piperacillin-tazobactam in pediatric non-renal solid organ transplantation. Pediatr Transplant 2007; 11:38-48.

993. Barker RJ, Mayes JT, Schulak JA. Wound abscesses following retroperitoneal pancreas transplantation. Clin Transplant 1991; 5:403-407.

994. Douzdjian V, Abecassis MM, Cooper JL et al. Incidence, management, and significance of surgical complications after pancreas-kidney transplantation. Surg Gynecol Obstet 1993; 177:451-456.

995. Everett JE, Wahoff DC, Statz C et al. Characterization and impact of wound infection after pancreas transplantation. Arch Surg 1994; 129:1310-1317. 
996. Ozaki CF, Stratta RJ, Taylor RJ et al. Surgical complications in solitary pancreas and combined pancreas-kidney transplantations. Am J Surg 1992; 164:546-551.

997. Sollinger HW, Ploeg RJ, Eckhoff DE et al. Two hundred consecutive simultaneous pancreas-kidney transplants with bladder drainage. Surgery 1993; 114:736-744.

998. United Network for Organ Sharing. Organ Procurement and Transplantation Network: data. http://optn.transplant .hrsa.gov/data/. Based on OPTN data as of October 2, 2008

999. Reddy KS, Stratta RJ, Shokouh-Amiri MH et al. Surgical complications after pancreas transplantation with portalenteric drainage. J Am Coll Surg 1999; 189:305-313.

1000. Berger N, Wirmsberger R, Kafka R et al. Infectious complications following 72 consecutive enteric-drained pancreas transplants. Transpl Int 2006; 19:549-557.

1001. Bonatti H, Berger $\bar{N}$, Kafka R et al. Experience with ATG short course high dose induction therapy in a series of 112 enteric drained pancreatic transplants. Ann Transplant 2002; 7:22-27.

1002. Berger N, Guggenbichler S, Steurer W et al. Bloodstream infection following 217 consecutive systemic-enteric drained pancreas transplants. BMC Infect Dis 2006; 6:127.

1003. Humar A, Kandawamy R, Drangstveit MB et al. Prolonged preservation increases surgical complications after pancreas transplants. Surgery 2000; 127:545-551.

1004. Pfundstein J, Roghmann MC, Schwalbe RS et al. A randomized trial of surgical antimicrobial prophylaxis with and without vancomycin in organ transplant patients. Clin Transplant 1999; 13:245-252.

1005. Smets YF, van der Pijl JW, van Dissel JT et al. Infectious disease complications of simultaneous pancreas-kidney transplantation. Nephrol Dial Transplant 1997; 12:764771.

1006. Michalak G, Kwiatkowski A, Bieniasz M et al. Infectious complications after simultaneous pancreas-kidney transplantation. Transplant Proc 2005; 37:3560-3563.

1007. Linhares MM, Gonzalez AM, Triviño T et al. Simultaneous pancreas-kidney transplantation: infectious complications and microbiological aspects. Transplant Proc 2004; 36:980-981.

1008. Bassetti M, Salvalaggio PR, Topal J et al. Incidence, timing and site of infections among pancreas transplant recipients. J Hosp Infect 2004; 56:184-190.

1009. Barone GW, Hudec WA, Sailors DM et al. Prophylactic wound antibiotics for combined kidney and pancreas transplants. Clin Transplant 1996; 10:386-388.

1010. Freise CE, Stock PG, Roberts JP et al. Low postoperative wound infection rates are possible following simultaneous pancreas-kidney transplantation. Transplant Proc 1995; 27:3069-3070.

1011. Smets YF, van der Pijl JW, van Dissel JT et al. Major bacterial and fungal infections after 50 simultaneous pancreas-kidney transplantations. Transplant Proc 1995; 27:3089-3090.

1012. Douzdjian V, Gugliuzza KK. Wound complications after simultaneous pancreas-kidney transplants: midline versus transverse incision. Transplant Proc 1995; 27:3130-3132.

1013. Bartlett ST. Pancreatic transplantation after thirty years: still room for improvement. J Am Coll Surg 1996; 183:408-410.

1014. Cohen J, Rees AJ, Williams G. A prospective randomized controlled trial of perioperative antibiotic prophylaxis in renal transplantation. J Hosp Infect 1988; 11:357-363.

1015. Hoy WE, May AG, Freeman RB. Primary renal transplant wound infections. N Y State J Med 1981; 81:1469-1473.
1016. Kohlberg WI, Tellis VA, Bhat DJ et al. Wound infections after transplant nephrectomy. Arch Surg 1980; 115:645646.

1017. Muakkassa WF, Goldman MH, Mendez-Picon G et al. Wound infections in renal transplant patients. J Urol 1983; 130:17-19.

1018. Novick AC. The value of intraoperative antibiotics in preventing renal transplant wound infections. J Urol 1981; 125:151-152.

1019. Ramos E, Karmi S, Alongi SV et al. Infectious complications in renal transplant recipients. South Med J 1980; 73:752-754.

1020. Rubin RH, Wolfson JS, Cosimi AB et al. Infection in the renal transplant recipient. Am J Med 1981; 70:405-411.

1021. Tilney NL, Strom TB, Vineyard GC et al. Factors contributing to the declining mortality rate in renal transplantation. N Engl J Med 1978; 299:1321-1325.

1022. Muñoz P. Management of urinary tract infections and lymphocele in renal transplant recipients. Clin Infect Dis 2001; 33(suppl 1):S53-S57.

1023. Alangaden GJ, Thyagarajan R, Gruber SA et al. Infectious complications after kidney transplantation: current epidemiology and associated risk factors. Clin Transplant 2006; 20:401-409.

1024. Dantas SP, Kuboyama RH, Mazzali M et al. Nosocomial infections in renal transplant patients: risk factors and treatment implications associated with urinary tract and surgical site infections. J Hosp Infect 2006; 63:117-123.

1025. Celik A, Sifil A, Cavdar C et al. Outcome of renal transplantation: 7-year experience. Transplant Proc 2001; 33: 2657-2659.

1026. Lai MK, Huang CC, Chu SH et al. Surgical complications in renal transplantation. Transplant Proc 1994; 26:2165-2166.

1027. Schmaldienst $S$, Hoerl WH. Bacterial infections after renal transplantation. Nephron 1997; 75:140-153.

1028. Maraha B, Bonten $\mathrm{H}$, van Hooff $\mathrm{H}$ et al. Infectious complications and antibiotic use in renal transplant recipients during a 1-year follow-up. Clin Microbiol Infect 2001; 7: 619-625.

1029. Ramos A, Asensio A, Muñoz E et al. Incisional surgical site infection in kidney transplantation. Urology 2008; 72: 119-123.

1030. Menezes FG, Wey SB, Peres CA et al. Risk factors for surgical site infection in kidney transplant recipients. Infect Control Hosp Epidemiol 2008; 29:771-773.

1031. Stephan RN, Munschauer CE, Kumar MS. Surgical wound infection in renal transplantation. Outcome data in 102 consecutive patients without perioperative systemic antibiotic coverage. Arch Surg 1997; 132:1315-1319.

1032. Sawyer RG, Pelletier SJ, Pruett TL. Increased early morbidity and mortality with acceptable long-term function in severely obese patients undergoing liver transplantation. Clin Transplant 1999; 13:126-130.

1033. Koyle MA, Glasscock RJ, Ward HJ et al. Declining incidence of wound infection in cadaveric renal transplant recipient. Urology 1988; 31:103-106.

1034. Judson RT. Wound infection following renal transplantation. Aust N Z J Surg 1984; 54:223-224.

1035. Del Rio G, Dalet F, Chechile G. Antimicrobial prophylaxis in urologic surgery: does it give some benefit? Eur Urol 1993; 24:305-312.

1036. Midtvedt K, Hartmann A, Midtvedt $\mathrm{T}$ et al. Routine perioperative antibiotic prophylaxis in renal transplantation. Nephrol Dial Transplant 1998; 13:1637-1641. 
1037. Capocasale E, Mazzoni MP, Tondo S et al. Antimicrobial prophylaxis with ceftriaxone in renal transplantation. Prospective study of 170 patients. Chemotherapy 1994; 40:435-440.

1038. Wakelin SJ, Casey J, Robertson A et al. The incidence and importance of bacterial contaminants of cadaveric renal perfusion fluid. Transplant Int 2005; 17:680-686.

1039. Zomorrodi A, Buhluli A. Is antibiotic usage necessary after donor nephrectomy? A single center experience. Saudi J Kidney Dis Transpl 2008; 19:200-205.

1040. Pape L, Offner G, Ehrich JH et al. A single center clinical experience in intensive care management of 104 pediatric renal transplantations between 1998 and 2002. Pediatr Transplant 2004; 8:39-43.

1041. Thorne $\mathrm{CH}$. Techniques and principles in plastic surgery. In: Thorne $\mathrm{CH}$, Beasley RW, Aston SJ et al., eds. Grabb and Smith's plastic surgery. 6th ed. Philadelphia: Lippincott Williams and Wilkins; 2007.

1042. Chełmoński A, Jabłecki J, Sycz Z. Composite allotransplantations of knee joint, larynx, uterus, abdominal wall, face and penis. Ann Transplant 2007; 12:5-11.

1043. Bonatti $H$, Brandacher $G$, Margreiter $R$ et al. Infectious complications in three double hand recipients: experience from a single center. Transplant Proc 2009; 41:517-520.

1044. Babcock MD, Grekin RC. Antibiotic use in dermatologic surgery. Dermatol Clin 2003; 21:337-348.

1045. Messingham MJ, Arpey CJ. Updates on the use of antibiotics in cutaneous surgery. Dermatol Surg 2005; 31:1068-1078.

1046. Wright TI, Baddour LM, Berbari EF et al. Antibiotic prophylaxis in dermatologic surgery: advisory statement 2008. J Am Acad Dermatol 2008; 59:464-473.

1047. Throckmorton AD, Boughey JC, Boostrom SY et al. Postoperative prophylactic antibiotics and surgical site infection rates in breast surgery patients. Ann Surg Oncol 2009; 16:2464-2469.

1048. Khan UD. Breast augmentation, antibiotic prophylaxis, and infection: comparative analysis of 1,628 primary augmentation mammoplasties assessing the role and efficacy of antibiotics prophylaxis duration. Aesthetic Plast Surg 2010; 34:42-47.

1049. Baran CN, Sensöz Ö, Ulusoy MG. Prophylactic antibiotics in plastic and reconstructive surgery. Plast Reconstr Surg 1999; 103:1561-1566.

1050. Mekako AI, Chetter IC, Coughlin PA et al., on behalf of the Hull Antibiotic pRophylaxis in varicose VEin Surgery Trialists (HARVEST). Randomized clinical trial of coamoxiclav versus no antibiotic prophylaxis in varicose vein surgery. Br J Surg 2010; 97:29-36.

1051. Stone JF, Davidson JS. The role of antibiotics and timing of repair in flexor tendon injuries of the hand. Ann Plast Surg 1998; 40:7-13.

1052. LeRoy J, Given KS. Wound infection in breast augmentation: the role of prophylactic perioperative antibiotics. Aesthetic Plast Surg 1991; 15:303-305.

1053. Stewart KJ, Stewart DA, Coghlan B et al. Complications of 278 consecutive abdominoplasties. J Plast Reconstr Aesth Surg 2006; 59:1152-1155.

1054. Rosengren H, Dixon A. Antibacterial prophylaxis in dermatologic surgery: an evidence-based review. Am J Clin Dermatol 2010; 11:35-44.

1055. Landes G, Harris PG, Lemaine V et al. Prevention of surgical site infection and appropriateness of antibiotic prescribing habits in plastic surgery. J Plast Reconstr Aesthet Surg 2008; 61:1347-1356.
1056. Ahmadi AH, Cohen BE, Shayani P. A prospective study of antibiotic efficacy in preventing infection in reduction mammaplasty. Plast Reconstr Surg 2005; 116:126-131.

1057. Carroll WR, Rosenstiel D, Fix JR et al. Three-dose vs. extended-course clindamycin prophylaxis for free-flap reconstruction of the head and neck. Arch Otolaryngol Head Neck Surg 2003; 129:771-774.

1058. Serletti JM, Davenport MS, Herrera HR et al. Efficacy of prophylactic antibiotics in reduction mammoplasty. Ann Plast Surg 1994; 33:476-480.

1059. Halpern AC, Leyden JJ, Dzubow LM et al. The incidence of bacteremia in skin surgery of the head and neck. I Am Acad Dermatol 1988; 19:112-116.

1060. Samra S, Sawh-Martinez R, Barry O et al. Complication rates of lipoabdominoplasty versus traditional abdominoplasty in high-risk patients. Plast Reconstr Surg 2010; 125:683-690.

1061. Olsen MA, Lefta M, Dietz JR et al. Risk factors for surgical site infection after major breast operation. J Am Coll Surg 2008; 207:326-335.

1062. Dixon AJ, Dixon MP, Dixon JB. Prospective study of skin surgery in patients with and without known diabetes. Dermatol Surg 2009; 35:1035-1040.

1063. Wahie S, Lawrence CM. Wound complications following diagnostic skin biopsies in dermatology inpatients. Arch Dermatol 2007; 143:1267-1271.

1064. Gravante G, Araco A, Sorge R et al. Wound infections in post-bariatric patients undergoing body contouring abdominoplasty: the role of smoking. Obes Surg 2007; 17:1325-1331.

1065. Rey JE, Gardner SM, Cushing RD. Determinants of surgical site infection after breast biopsy. Am J Infect Control 2005; 33:126-129.

1066. Sevin A, Senen D, Sevin K et al. Antibiotic use in abdominoplasty: prospective analysis of 207 cases. J Plast Reconstr Aesthet Surg 2007; 60:379-382.

1067. Gravante G, Caruso R, Araco A et al. Infections after plastic procedures: incidences, etiologies, risk factors, and antibiotic prophylaxis. Aesthetic Plast Surg 2008; 32:243-251.

1068. Bertin ML, Crowe J, Gordon SM. Determinants of surgical site infection after breast surgery. Am J Infect Control 1998; 26:61-65.

1069. Harness NG, Inacio MC, Pfeil FF et al. Rate of infection after carpal tunnel release surgery and effect of antibiotic prophylaxis. J Hand Surg 2010; 35:189-196.

1070. Platt R, Zucker JR, Zaleznik DF et al. Perioperative antibiotic prophylaxis and wound infection following breast surgery. J Antimicrob Chemother 1993; 31(suppl B):43-48.

1071. Kompatscher P, von Planta A, Spicher I et al. Comparison of the incidence and predicted risk of early surgical site infections after breast reduction. Aesthetic Plast Surg 2003; 27:308-314.

1072. Bunn F, Cunningham ME, Handscomb K. Prophylactic antibiotics to prevent surgical site infection after breast cancer surgery. Cochrane Database Syst Rev 2006; 2:CD005360.

1073. Perrotti JA, Castor SA, Perez PC et al. Antibiotic use in aesthetic surgery: a national survey and literature review. Plast Reconstr Surg 2002; 109:1685-1693.

1074. Smyth AG, Knepil GJ. Prophylactic antibiotics and surgery for primary clefts. Br J Oral Maxillofac Surg 2008; 46: 107-109.

1075. Cocco JF, Antonetti JW, Burns JL et al. Characterization of the nasal, sublingual and oropharyngeal mucosa microbiota in cleft lip and palate individuals before and after surgical repair. Cleft Palate Craniofac J 2010; 47:151-155.

(Appendices follow $\rightarrow$ ) 


\section{Appendix A. National Healthcare Safety Network Criteria} FOR CLASSIFYING WOUNDS ${ }^{35}$

\section{Clean}

An uninfected operative wound in which no inflammation is encountered and the respiratory, alimentary, genital, or uninfected urinary tracts are not entered. In addition, clean wounds are primarily closed and, if necessary, drained with closed drainage. Operative incisional wounds that follow nonpenetrating (blunt) trauma should be included in this category if they meet the criteria.

\section{Clean-contaminated}

Operative wounds in which the respiratory, alimentary, genital, or urinary tracts are entered under controlled conditions and without unusual contamination. Specifically, operations involving the biliary tract, appendix, vagina, and oropharynx are included in this category, provided no evi- dence of infection or major break in technique is encountered.

\section{Contaminated}

Open, fresh, accidental wounds. In addition, operations with major breaks in sterile technique (e.g., open cardiac massage) or gross spillage from the gastrointestinal tract and incisions in which acute, nonpurulent inflammation is encountered are included in this category.

\section{Dirty or infected}

Includes old traumatic wounds with retained devitalized tissue and those that involve existing clinical infection or perforated viscera. This definition suggests that the organisms causing postoperative infection were present in the operative field before the operation.

\section{Appendix B. National Healthcare Safety Network Criteria} for Defining a Surgical-Site Infection (SSI) $)^{8,36}$

\section{Superficial incisional SSI}

Occurs within 30 days postoperatively and involves skin or subcutaneous tissue of the incision and at least one of the following: (1) purulent drainage from the superficial incision, (2) organisms isolated from an aseptically obtained culture of fluid or tissue from the superficial incision, (3) at least one of the following signs or symptoms of infection: pain or tenderness, localized swelling, redness, or heat, and superficial incision is deliberately opened by surgeon and is culturepositive or not cultured (a culture-negative finding does not meet this criterion), and (4) diagnosis of superficial incisional SSI by the surgeon or attending physician.

\section{Deep incisional SSI}

Occurs within 30 days after the operative procedure if no implant is left in place or within one year if implant is in place and the infection appears to be related to the operative procedure, involves deep soft tissues (e.g., fascial and muscle layers) of the incision, and the patient has at least one of the following: (1) Purulent drainage from the deep incision but not from the organ/space component of the surgical site, (2) a deep incision spontaneously dehisces or is deliberately opened by a surgeon and is culture-positive or not cultured and the patient has at least one of the following signs or symptoms: fever $\left(>38^{\circ} \mathrm{C}\right)$ or localized pain or tenderness (a culture-negative finding does not meet this criterion), (3) an abscess or other evidence of infection involving the deep incision is found on direct examination, during reoperation, or by histopathologic or radiologic examination, and (4) diagnosis of a deep incisional SSI by a surgeon or attending physician.

\section{Organ/space SSI}

Involves any part of the body, excluding the skin incision, fascia, or muscle layers, that is opened or manipulated during the operative procedure. Specific sites are assigned to organ/ space SSI to further identify the location of the infection (e.g., endocarditis, endometritis, mediastinitis, vaginal cuff, and osteomyelitis). Organ/space SSI must meet the following criteria: (1) Infection occurs within 30 days after the operative procedure if no implant is in place or within one year if implant is in place and the infection appears to be related to the operative procedure, (2) infection involves any part of the body, excluding the skin incision, fascia, or muscle layers, that is opened or manipulated during the operative procedure, and (3) the patient has at least one of the following: (a) purulent drainage from a drain that is placed through a stab wound into the organ/space, (b) organisms isolated from an aseptically obtained culture of fluid or tissue in the organ/space, (c) an abscess or other evidence of infection involving the organ/space that is found on direct examination, during reoperation, or by histopathologic or radiologic examination, and (d) diagnosis of an organ/space SSI by a surgeon or attending physician.

Address correspondence to: Dr. Dale W. Bratzler The University of Oklahoma Health Sciences Center 801 Northeast 13th St., Room 135 PO Box 26901

Oklahoma City, OK 73126-0901

E-mail: Dale-bratzler@ouhsc.edu 


\section{This article has been cited by:}

1. Maya Hites, Fabio Silvio Taccone. Dosing in Obese Critically Ill Patients 47-72. [Crossref]

2. Joseph A. Dottino, Karen H. Lu, Melinda S. Yates. Management of Endometrial Cancer Precursors in Obese Women 189-212. [Crossref]

3. Andrew Krause, Zain Sayeed, Mouhanad El-Othmani, Vinay Pallekonda, William Mihalko, Khaled J. Saleh. 2018. Outpatient Total Knee Arthroplasty. Orthopedic Clinics of North America 49:1, 7-16. [Crossref]

4. Alfonso Vaquero-Picado, E. Carlos Rodríguez-Merchán. Antibiotic Prophylaxis to Prevent Infection in Total Knee Arthroplasty 35-46. [Crossref]

5. Itai Gans, Amit Jain, Norachart Sirisreetreerux, Elliott R. Haut, Erik A. Hasenboehler. 2017. Current practice of antibiotic prophylaxis for surgical fixation of closed long bone fractures: a survey of 297 members of the Orthopaedic Trauma Association. Patient Safety in Surgery 11:1. . [Crossref]

6. Alexander P. Glaser, Ilina Rosoklija, Emilie K. Johnson, Elizabeth B. Yerkes. 2017. Prophylactic antibiotic use in pediatric patients undergoing urinary tract catheterization: a survey of members of the Society for Pediatric Urology. BMC Urology 17:1. . [Crossref]

7. Willem-Jan Metsemakers, Jolien Onsea, Emilie Neutjens, Ester Steffens, Annette Schuermans, Martin McNally, Stefaan Nijs. 2017. Prevention of fracture-related infection: a multidisciplinary care package. International Orthopaedics 41:12, 2457-2469. [Crossref]

8. Melissa H. Lippitt, Amanda N. Fader, MaryAnn B. Wilbur. 2017. Preventable Surgical Harm in Gynecologic Oncology: Optimizing Quality and Patient Safety. Current Obstetrics and Gynecology Reports 6:4, 298-309. [Crossref]

9. Sarah Walker, Ankur Datta, Roxanne L. Massoumi, Erica R. Gross, Michael Uhing, Marjorie J. Arca. 2017. Antibiotic stewardship in the newborn surgical patient: A quality improvement project in the neonatal intensive care unit. Surgery 162:6, 1295-1303. [Crossref]

10. Margot E. Cohen, Hojjat Salmasian, Jianhua Li, Jianfang Liu, Philip Zachariah, Jason D. Wright, Daniel E. Freedberg. 2017. Surgical Antibiotic Prophylaxis and Risk for Postoperative Antibiotic-Resistant Infections. Journal of the American College of Surgeons 225:5, 631-638.e3. [Crossref]

11. Rhae Riegel, Kevin Sweeney, Gino Inverso, Peter Quinn, Eric J. Granquist. 2017. Microbiology and Associated Risk Factors in Alloplastic Total Joint Infections: A 20-Year Retrospective Study. Journal of Oral and Maxillofacial Surgery . [Crossref]

12. Christopher R. Ingraham, Guy E. Johnson, Emily L. Albrecht, Siddharth A. Padia, Eric J. Monroe, Brandon C. Perry, Ethan M. Dobrow, Daniel S. Hippe, Karim Valji. 2017. Value of Antibiotic Prophylaxis for Percutaneous Gastrostomy: A Double-Blind Randomized Trial. Journal of Vascular and Interventional Radiology . [Crossref]

13. Yarah M. Haidar, Prem B. Tripathi, Tjoson Tjoa, Sartaaj Walia, Lishi Zhang, Yanjun Chen, Danh V. Nguyen, Hossein Mahboubi, William B. Armstrong, Julie A. Goddard. 2017. Antibiotic prophylaxis in clean-contaminated head and neck cases with microvascular free flap reconstruction: A systematic review and meta-analysis. Head \& Neck 8. . [Crossref]

14. Marie Strøm Zangenberg, Nir Horesh, Uri Kopylov, Alaa El-Hussuna. 2017. Preoperative optimization of patients with inflammatory bowel disease undergoing gastrointestinal surgery: a systematic review. International Journal of Colorectal Disease 14. . [Crossref]

15. E Patchen Dellinger, Rupali Jain, Paul S Pottinger. 2017. The Influence of Reported Penicillin Allergy. Clinical Infectious Diseases . [Crossref]

16. Kimberly G Blumenthal, Erin E Ryan, Yu Li, Hang Lee, James L Kuhlen, Erica S Shenoy. 2017. The Impact of a Reported Penicillin Allergy on Surgical Site Infection Risk. Clinical Infectious Diseases . [Crossref]

17. M. Vallée, F. Bruyère, F. Roblot, L. Brureau. 2017. Place de la témocilline dans le traitement des infections urinaires. Progrès en Urologie 27:12, 609-617. [Crossref]

18. Mark Dornfeld, Jenna K. Lovely, Marianne Huebner, David W. Larson. 2017. Surgical Site Infection in Colorectal Surgery. Diseases of the Colon \& Rectum 60:9, 971-978. [Crossref]

19. Donghua Xie, Victor Gheiler, Isabel Lopez, Guy M. Nehrenz, Boris Klopukh, Fernando Bianco, Paul Perito, Edward Gheiler. 2017. Experience With Prophylactic Gentamicin During Penile Prosthesis Surgery: A Retrospective Comparison of Two Different Doses. The Journal of Sexual Medicine 14:9, 1160-1164. [Crossref]

20. Sumanth Gandra, Sanjeev Singh, Dasaratha Jinka, Ravishankar Kanithi, Ashok Chikkappa, Anita Sharma, Dhanya Dharmapalan, Anil Vasudevan, Onkaraiah Tunga, Akhila Akula, Garima Garg, Yingfen Hsia, Srinivas Murki, Gerardo Alvarez-Uria, Mike Sharland, Ramanan Laxminarayan. 2017. Point Prevalence Surveys of Antimicrobial Use among Hospitalized Children in Six Hospitals in India in 2016. Antibiotics 6:3, 19. [Crossref] 
21. Jesse Zanker, Gustavo Duque. 2017. Rapid Geriatric Assessment of Hip Fracture. Clinics in Geriatric Medicine 33:3, 369-382. [Crossref]

22. Hiroko Machida, Marianne S. Hom, Anastasiya Shabalova, Brendan H. Grubbs, Koji Matsuo. 2017. Predictive model of urinary tract infection after surgical treatment for women with endometrial cancer. Archives of Gynecology and Obstetrics 296:2, 335-343. [Crossref]

23. Shitanshu Uppal, Ali Bazzi, R. Kevin Reynolds, John Harris, Mark D. Pearlman, Darrell A. Campbell, Daniel M. Morgan. 2017. Chlorhexidine-Alcohol Compared With Povidone-Iodine for Preoperative Topical Antisepsis for Abdominal Hysterectomy. Obstetrics \& Gynecology 130:2, 319-327. [Crossref]

24. Lorie M. Harper, Meredith Kilgore, Jeff M. Szychowski, William W. Andrews, Alan T. N. Tita. 2017. Economic Evaluation of Adjunctive Azithromycin Prophylaxis for Cesarean Delivery. Obstetrics \& Gynecology 130:2, 328-334. [Crossref]

25. Assawapalanggool Srisuda, Kasatpibal Nongyao, Sirichotiyakul Supatra, Arora Rajin, Suntornlimsiri Watcharin. 2017. A Prognostic Scoring Tool for Cesarean Organ/Space Surgical Site Infections: Derivation and Internal Validation. Surgical Infections 18:6, 694-701. [Abstract] [Full Text HTML] [Full Text PDF] [Full Text PDF with Links]

26. Simona Silvetti, Marco Ranucci, Giuseppe Isgrò, Valentina Villa, Elena Costa. 2017. Preoperative colonization in pediatric cardiac surgery and its impact on postoperative infections. Pediatric Anesthesia 27:8, 849-855. [Crossref]

27. Huston Jared M., Kao Lillian S., Chang Phillip K., Sanders James M., Buckman Sara, Adams Charles A., Cocanour Christine S., Parli Sarah E., Grabowski Julia, Diaz Jose, Tessier Jeffrey M., Duane Therese M.. 2017. Antibiotics vs. Appendectomy for Acute Uncomplicated Appendicitis in Adults: Review of the Evidence and Future Directions. Surgical Infections 18:5, 527-535. [Abstract] [Full Text HTML] [Full Text PDF] [Full Text PDF with Links]

28. Sarkut Pinar, Kilicturgay Sadik, Aktas Hikmet, Ozen Yilmaz, Kaya Ekrem. 2017. Routine Use of Prophylactic Antibiotics during Laparoscopic Cholecystectomy Does Not Reduce the Risk of Surgical Site Infections. Surgical Infections 18:5, 603-609. [Abstract] [Full Text HTML] [Full Text PDF] [Full Text PDF with Links]

29. E. Charani, R. Ahmad, C. Tarrant, G. Birgand, A. Leather, M. Mendelson, S.R. Moonesinghe, N. Sevdalis, S. Singh, A. Holmes. 2017. Opportunities for system level improvement in antibiotic use across the surgical pathway. International Journal of Infectious Diseases 60, 29-34. [Crossref]

30. Kei Nakashima, Masahiro Aoshima, Tamao Nakashita, Masahiko Hara, Ayumu Otsuki, Satoshi Noma, Masafumi Misawa, Yoshihito Otsuka, Shinji Motojima. 2017. Low-dose trimethoprim-sulfamethoxazole treatment for pneumocystis pneumonia in non-human immunodeficiency virus-infected immunocompromised patients: A single-center retrospective observational cohort study. Journal of Microbiology, Immunology and Infection . [Crossref]

31. Stijn Willem de Jonge, Sarah L. Gans, Jasper J. Atema, Joseph S. Solomkin, Patchen E. Dellinger, Marja A. Boermeester. 2017. Timing of preoperative antibiotic prophylaxis in 54,552 patients and the risk of surgical site infection. Medicine 96:29, e6903. [Crossref]

32. Alon Vaisman, Janine McCready, Sandy Hicks, Jeff Powis. 2017. Optimizing preoperative prophylaxis in patients with reported $\beta$-lactam allergy: a novel extension of antimicrobial stewardship. Journal of Antimicrobial Chemotherapy . [Crossref]

33. B. I. Naik, C. Roger, K. Ikeda, M. S. Todorovic, S. C. Wallis, J. Lipman, J. A. Roberts. 2017. Comparative total and unbound pharmacokinetics of cefazolin administered by bolus versus continuous infusion in patients undergoing major surgery: a randomized controlled trial. BJA: British Journal of Anaesthesia 118:6, 876-882. [Crossref]

34. Yiying Cai, Indumathi Venkatachalam, Nancy W. Tee, Thean Yen Tan, Asok Kurup, Sin Yew Wong, Chian Yong Low, Yang Wang, Winnie Lee, Yi Xin Liew, Brenda Ang, David C. Lye, Angela Chow, Moi Lin Ling, Helen M. Oh, Cassandra A. Cuvin, Say Tat Ooi, Surinder K. Pada, Chong Hee Lim, Jack Wei Chieh Tan, Kean Lee Chew, Van Hai Nguyen, Dale A. Fisher, Herman Goossens, Andrea L. Kwa, Paul A. Tambyah, Li Yang Hsu, Kalisvar Marimuthu. 2017. Prevalence of Healthcare-Associated Infections and Antimicrobial Use Among Adult Inpatients in Singapore Acute-Care Hospitals: Results From the First National Point Prevalence Survey. Clinical Infectious Diseases 64:suppl_2, S61-S67. [Crossref]

35. Giorgio Tarchini, Kui Hin Liau, Joseph S. Solomkin. 2017. Antimicrobial Stewardship in Surgery: Challenges and Opportunities. Clinical Infectious Diseases 64:suppl_2, S112-S114. [Crossref]

36. Nongyao Kasatpibal, JoAnne D. Whitney, Surasak Saokaew, Kirati Kengkla, Margaret M. Heitkemper, Anucha Apisarnthanarak. 2017. Effectiveness of Probiotic, Prebiotic, and Synbiotic Therapies in Reducing Postoperative Complications: A Systematic Review and Network Meta-analysis. Clinical Infectious Diseases 64:suppl_2, S153-S160. [Crossref]

37. Nirmal Tejwani, Kevin Klifto, Christopher Looze, Christopher Scott Klifto. 2017. Treatment of Pregnant Patients With Orthopaedic Trauma. Journal of the American Academy of Orthopaedic Surgeons 25:5, e90-e101. [Crossref]

38. E. Patchen Dellinger. 2017. Glycopeptides Versus Beta-lactams for the Prevention of Surgical Site Infections in Cardiovascular and Orthopedic Surgery. Annals of Surgery 265:5, e70-e71. [Crossref] 
39. Kasatpibal Nongyao, Whitney Joanne D., Dellinger E. Patchen, Nair Bala G., Pike Kenneth C.. 2017. Failure to Redose Antibiotic Prophylaxis in Long Surgery Increases Risk of Surgical Site Infection. Surgical Infections 18:4, 474-484. [Abstract] [Full Text HTML] [Full Text PDF] [Full Text PDF with Links]

40. Amal F. Alotaibi, Rania A. Mekary, Hasan A. Zaidi, Timothy R. Smith, Ankur Pandya. 2017. Safety and Efficacy of Antibacterial Prophylaxis After Craniotomy: A Decision Model Analysis. World Neurosurgery . [Crossref]

41. Goldberg Stephanie R., Henning Jennifer, Wolfe Luke G., Duane Therese M.. 2017. Practice Patterns for the Use of Antibiotic Agents in Damage Control Laparotomy and Its Impact on Outcomes. Surgical Infections 18:3, 282-286. [Abstract] [Full Text HTML] [Full Text PDF] [Full Text PDF with Links]

42. Alias David, Ruiz-Tovar Jaime, Moreno Ana, Manso Belen, Diaz Gustavo, Duran Manuel, Garcia-Olmo Damian. 2017. Effect of Subcutaneous Sterile Vitamin E Ointment on Incisional Surgical Site Infection after Elective Laparoscopic Colorectal Cancer Surgery. Surgical Infections 18:3, 287-292. [Abstract] [Full Text HTML] [Full Text PDF] [Full Text PDF with Links]

43. Katherine H. Chan, Teresa Bell, Mark Cain, Aaron Carroll, Brian D. Benneyworth. 2017. Variation in Surgical Antibiotic Prophylaxis for Outpatient Pediatric Urological Procedures at United States Children's Hospitals. The Journal of Urology 197:3, 944-950. [Crossref]

44. Aurora Pop-Vicas, Jackson S. Musuuza, Michelle Schmitz, Ahmed Al-Niaimi, Nasia Safdar. 2017. Incidence and risk factors for surgical site infection post-hysterectomy in a tertiary care center. American Journal of Infection Control 45:3, 284-287. [Crossref]

45. Laurie J. Conway, Shanina C. Knighton. 2017. Journal club: Social media as an antimicrobial stewardship tool. American Journal of Infection Control 45:3, 293-294. [Crossref]

46. Rose-Marie Scarlato, Michelle M. Dowsey, Kirsty L. Buising, Peter F. M. Choong, Trisha N. Peel. 2017. What is the role of catheter antibiotic prophylaxis for patients undergoing joint arthroplasty?. ANZ Journal of Surgery 87:3, 153-158. [Crossref]

47. Stefanie Saunders, Stephen Reese, Jimmy Lam, Jacqueline Wulu, Scharkuh Jalisi, Waleed Ezzat. 2017. Extended use of perioperative antibiotics in head and neck microvascular reconstruction. American Journal of Otolaryngology 38:2, 204-207. [Crossref]

48. Akshay Sood, Firas Abdollah, Jesse D. Sammon, Nivedita Arora, Matthew Weeks, James O. Peabody, Mani Menon, Quoc-Dien Trinh. 2017. Postoperative sepsis prediction in patients undergoing major cancer surgery. Journal of Surgical Research 209, 60-69. [Crossref]

49. Gabriel M. Klein, Brett T. Phillips, Alexander B. Dagum, Duc T. Bui, Sami U. Khan. 2017. Infectious Loss of Tissue Expanders in Breast Reconstruction. Annals of Plastic Surgery 78:2, 149-152. [Crossref]

50. R.P.D. Cooke, B. Pettorini, C. Mallucci. 2017. Prevention and management of neurosurgical shunt infections. Journal of Hospital Infection 95:2, 161-162. [Crossref]

51. Devin M. Williams, Andy O. Miller, Michael W. Henry, Geoffrey H. Westrich, Hassan M.K. Ghomrawi. 2017. Cost-Effectiveness of Staphylococcus aureus Decolonization Strategies in High-Risk Total Joint Arthroplasty Patients. The Journal of Arthroplasty . [Crossref]

52. Patricia López Pereira, Cristina Díaz-Agero Pérez, Nieves López Fresneña, Julio Las Heras Mosteiro, Aurelio Palancar Cabrera, Ángela Lourdes Rincón Carlavilla, Jesús María Aranaz Andrés. 2017. 'Epidemiology of surgical site infection in a neurosurgery department'. British Journal of Neurosurgery 31:1, 10-15. [Crossref]

53. Heather L. Evans, Eileen M. Bulger. Infectious Complications Following Surgery and Trauma 684-692.e2. [Crossref]

54. Itzhak Brook. Anaerobic Bacteria 1628-1644.e2. [Crossref]

55. Reem Almaghrabi, Cornelius J. Clancy, M. Hong Nguyen. Prevention of Perioperative Infections in Organ Transplant Recipients 11-24. [Crossref]

56. Mary Elizabeth Sexton, Jesse T. Jacob. 2017. Commentary on: Incidence and Risk Factors for Major Surgical Site Infections in Aesthetic Surgery: Analysis of 129,007 Patients. Aesthetic Surgery Journal 37:1, 100-102. [Crossref]

57. M.G. Desale, E.J. Tanner, A.K. Sinno, A. Africano Angarita, A.N. Fader, R.L. Stone, K.L. Levinson, R.E. Bristow, K. Long Roche. 2017. Perioperative fluid status and surgical outcomes in patients undergoing cytoreductive surgery for advanced epithelial ovarian cancer. Gynecologic Oncology 144:1, 61-64. [Crossref]

58. Erik J. Teicher, Christopher P. Michetti. Nutrition, Antibiotics, and Post-traumatic Seizure Prophylaxis 239-246. [Crossref]

59. Mark A. Malangoni. Surgical Site Infections and Their Management 167-185. [Crossref]

60. Mazuski John E., Tessier Jeffrey M., May Addison K., Sawyer Robert G., Nadler Evan P., Rosengart Matthew R., Chang Phillip K., O'Neill Patrick J., Mollen Kevin P., Huston Jared M., Diaz Jose J. Jr, Prince Jose M.. 2017. The Surgical Infection Society Revised Guidelines on the Management of Intra-Abdominal Infection. Surgical Infections 18:1, 1-76. [Abstract] [Full Text HTML] [Full Text PDF] [Full Text PDF with Links] [Supplemental Material] 
61. A.O. Miller, M.W. Henry, B.D. Brause. Prevention of joint infections 3-23. [Crossref]

62. Lizabeth D. Martin, Michael Kallile, Siri Kanmanthreddy, Danielle M. Zerr, Mark Thomas. 2017. Infection prevention in pediatric anesthesia practice. Pediatric Anesthesia 27:11, 1077. [Crossref]

63. 2017. OUP accepted manuscript. European Journal of Cardio-Thoracic Surgery . [Crossref]

64. Zachary David Levy. Exam 3 Questions 95-141. [Crossref]

65. Michelle Butterworth, Trevor Payne. Surgical Infections 69-87. [Crossref]

66. Kishiko Ikehara, Shungo Endo, Kensuke Kumamoto, Eiji Hidaka, Fumio Ishida, Jun-ichi Tanaka, Shin-ei Kudo. 2016. Positive detection of exfoliated colon cancer cells on linear stapler cartridges was associated with depth of tumor invasion and preoperative bowel preparation in colon cancer. World Journal of Surgical Oncology 14:1. . [Crossref]

67. Kelly Yamasato, Kurt Yoshino, Ann L. Chang, Aaron B. Caughey, Pai-Jong Tsai. 2016. Cesarean delivery complications in women with morbid obesity. The Journal of Maternal-Fetal \& Neonatal Medicine 29:23, 3885-3888. [Crossref]

68. Joshua G. Barton. 2016. Enhanced Recovery Pathways in Pancreatic Surgery. Surgical Clinics of North America 96:6, 1301-1312. [Crossref]

69. Rafael Franco-Cendejas, Erika Lizbeth Contreras-Córdova, Jaime Arturo Mondragón-Eguiluz, Edgar Samuel Vanegas-Rodríguez, Víctor Manuel Ilizaliturri-Sánchez, Arturo Galindo-Fraga. 2016. Incidencia de infecciones protésicas primarias de cadera y rodilla en un centro de la Ciudad de México. Cirugía y Cirujanos. [Crossref]

70. Benedetta Allegranzi, Peter Bischoff, Stijn de Jonge, N Zeynep Kubilay, Bassim Zayed, Stacey M Gomes, Mohamed Abbas, Jasper J Atema, Sarah Gans, Miranda van Rijen, Marja A Boermeester, Matthias Egger, Jan Kluytmans, Didier Pittet, Joseph S Solomkin. 2016. New WHO recommendations on preoperative measures for surgical site infection prevention: an evidence-based global perspective. The Lancet Infectious Diseases 16:12, e276-e287. [Crossref]

71. Astrid P. Uyttebroek, Ine I. Decuyper, Chris H. Bridts, Antonino Romano, Margo M. Hagendorens, Didier G. Ebo, Vito Sabato. 2016. Cefazolin Hypersensitivity: Toward Optimized Diagnosis. The Journal of Allergy and Clinical Immunology: In Practice 4:6, 1232-1236. [Crossref]

72. Lotte Jakobsen, Katrin G. Kuhn, Frank Hansen, Robert L. Skov, Anette M. Hammerum, Pia J. Littauer, Ole Thorlacius-Ussing, Peter H. Gebuhr, Jenny D. Knudsen, Henrik C. Schønheyder. 2016. Fecal carriage of extended-spectrum and AmpC $\beta$-lactamaseproducing Enterobacteriaceae in surgical patients before and after antibiotic prophylaxis. Diagnostic Microbiology and Infectious Disease 86:3, 316-321. [Crossref]

73. J. Alexander Viehman, Cornelius J. Clancy, Lloyd Clarke, Ryan K. Shields, Fernanda P. Silveira, Eun J. Kwak, Pascalis Vergidis, Christopher Hughes, Abhinav Humar, M. Hong Nguyen. 2016. Surgical Site Infections After Liver Transplantation. Transplantation 100:10, 2107-2114. [Crossref]

74. Jeffrey J. Houlton, Scott E. Bevans, Neal D. Futran. 2016. Unfavorable Results After Free Tissue Transfer to Head and Neck. Clinics in Plastic Surgery 43:4, 683-693. [Crossref]

75. Junko Okada, Yukiko Yamamizu, Kiyoko Fukai. 2016. Effectiveness of hand hygiene depends on the patient's health condition and care environment. Japan Journal of Nursing Science 13:4, 413-423. [Crossref]

76. Brett T. Phillips, Eric G. Halvorson. 2016. Antibiotic Prophylaxis following Implant-Based Breast Reconstruction. Plastic and Reconstructive Surgery 138:4, 751-757. [Crossref]

77. Suleyman Ozdemir, Kamil Gulpinar, S. Erpulat Ozis, Zafer Sahli, S. Altug Kesikli, Atila Korkmaz, I. Ethem Gecim. 2016. The effects of preoperative oral antibiotic use on the development of surgical site infection after elective colorectal resections: A retrospective cohort analysis in consecutively operated 90 patients. International Journal of Surgery 33, 102-108. [Crossref]

78. Dellinger E. Patchen. 2016. Prevention of Hospital-Acquired Infections. Surgical Infections 17:4, 422-426. [Abstract] [Full Text HTML] [Full Text PDF] [Full Text PDF with Links]

79. Michelle Louie, Tarek Toubia, Lauren D. Schiff. 2016. Considerations for minimally invasive gynecologic surgery in obese patients. Current Opinion in Obstetrics and Gynecology 28:4, 283-289. [Crossref]

80. J. Dawod, A. Tager, R.O. Darouiche, M. Al Mohajer. 2016. Prevention and management of internal cerebrospinal fluid shunt infections. Journal of Hospital Infection 93:4, 323-328. [Crossref]

81. Jashvant Poeran, Isaac Wasserman, Nicole Zubizarreta, Madhu Mazumdar. 2016. Characteristics of Antibiotic Prophylaxis and Risk of Surgical Site Infections in Open Colectomies. Diseases of the Colon \& Rectum 59:8, 733-742. [Crossref]

82. Ruston S. Taylor, Lara S. Shekerdemian. 2016. Avoidance of Hospital-Acquired Infections in Pediatric Cardiac Surgical Patients. Pediatric Critical Care Medicine 17, S279-S286. [Crossref]

83. Cedric Hunter, Lorne Rosenfield, Elena Silverstein, Panayiota Petrou-Zeniou. 2016. Methicillin-Resistant Staphylococcus aureus Infections. Plastic and Reconstructive Surgery 138:2, 515-523. [Crossref] 
84. Hiroshi Morioka, Aki Hirabayashi, Mitsutaka Iguchi, Yuka Tomita, Daizo Kato, Naokazu Sato, Miyuki Hyodo, Naoko Kawamura, Takuya Sadomoto, Kazuya Ichikawa, Takayuki Inagaki, Yoshiaki Kato, Yuichi Kouyama, Yoshinori Ito, Tetsuya Yagi. 2016. The first point prevalence survey of health care-associated infection and antimicrobial use in a Japanese university hospital: A pilot study. American Journal of Infection Control 44:7, e119-e123. [Crossref]

85. Ashraf F Nabhan, Nahed E Allam, Mohamed Hamed Abdel-Aziz Salama. Routes of administration of antibiotic prophylaxis for preventing infection after caesarean section . [Crossref]

86. Brett T. Phillips, Mitchell S. Fourman, Muath Bishawi, Mary Zegers, Brian J. O'Hea, Jason C. Ganz, Tara L. Huston, Alexander B. Dagum, Sami U. Khan, Duc T. Bui. 2016. Are Prophylactic Postoperative Antibiotics Necessary for Immediate Breast Reconstruction? Results of a Prospective Randomized Clinical Trial. Journal of the American College of Surgeons 222:6, 1116-1124. [Crossref]

87. Amos Adler, David E. Katz, Dror Marchaim. 2016. The Continuing Plague of Extended-spectrum $\beta$-lactamase-producing Enterobacteriaceae Infections. Infectious Disease Clinics of North America 30:2, 347-375. [Crossref]

88. Kevin L. Grimes, Haruhiro Inoue. 2016. Per Oral Endoscopic Myotomy for Achalasia. Thoracic Surgery Clinics 26:2, 147-162. [Crossref]

89. N. Wetzstein, H.-R. Brodt. 2016. Perioperative Antiinfektivaprophylaxe in der Kardiochirurgie. Zeitschrift für Herz-, Thorax- und Gefäßchirurgie 30:2, 120-126. [Crossref]

90. P A Prasad, J Wong-McLoughlin, S Patel, S E Coffin, T E Zaoutis, J Perlman, P DeLaMora, L Alba, Y-h Ferng, L Saiman. 2016. Surgical site infections in a longitudinal cohort of neonatal intensive care unit patients. Journal of Perinatology 36:4, 300-305. [Crossref]

91. Yanet Pedroso-Fernandez, Armando Aguirre-Jaime, Maria J. Ramos, Miriam Hernández, Milagros Cuervo, Alberto Bravo, Angel Carrillo. 2016. Prediction of surgical site infection after colorectal surgery. American Journal of Infection Control 44:4, 450-454. [Crossref]

92. Masaru Matsumura, Akio Saiura, Yosuke Inoue, Takeaki Ishizawa, Yoshihiro Mise, Yu Takahashi. 2016. High Rate of Organ/Space Surgical Site Infection After Hepatectomy with Preexisting Bilioenteric Anastomosis. World Journal of Surgery 40:4, 937-945. [Crossref]

93. Wael Mohamed Mohamed Moussa, Mohamed Abbas Aly Mohamed. 2016. Efficacy of postoperative antibiotic injection in and around ventriculoperitoneal shunt in reduction of shunt infection: A randomized controlled trial. Clinical Neurology and Neurosurgery 143, 144-149. [Crossref]

94. Katharina Meszaros, Urs Fuehrer, Sina Grogg, Gottfried Sodeck, Martin Czerny, Jonas Marschall, Thierry Carrel. 2016. Risk Factors for Sternal Wound Infection After Open Heart Operations Vary According to Type of Operation. The Annals of Thoracic Surgery 101:4, 1418-1425. [Crossref]

95. Jennifer R. Lyden, E. Patchen Dellinger. 2016. Surgical Site Infections. Hospital Medicine Clinics 5:2, 319-333. [Crossref]

96. Yotam Shkedy, Uri Alkan, Benjamin R. Roman, Ohad Hilly, Raphael Feinmesser, Gideon Bachar, Aviram Mizrachi. 2016. Role of perioperative antibiotic treatment in parotid gland surgery. Head \& Neck 38:S1, E1876-E1880. [Crossref]

97. David B. Banach, David R. Peaper, Brett E. Fortune, Sukru Emre, Louise M. Dembry. 2016. The clinical and molecular epidemiology of pre-transplant vancomycin-resistant enterococci colonization among liver transplant recipients. Clinical Transplantation 30:3, 306-311. [Crossref]

98. S. E. Salem, C. J. Proudman, D. C. Archer. 2016. Prevention of post operative complications following surgical treatment of equine colic: Current evidence. Equine Veterinary Journal 48:2, 143-151. [Crossref]

99. Jinbeom Cho, Ilyoung Park, Dosang Lee, Kiyoung Sung, Jongmin Baek, Junhyun Lee. 2016. Antimicrobial treatment after laparoscopic appendectomy for preventing a post-operative intraabdominal abscess: A Prospective Cohort Study of 1817 patients. International Journal of Surgery 27, 142-146. [Crossref]

100. Jashvant Poeran, Madhu Mazumdar, Rehana Rasul, Joanne Meyer, Henry S. Sacks, Brian S. Koll, Frances R. Wallach, Alan Moskowitz, Annetine C. Gelijns. 2016. Antibiotic prophylaxis and risk of Clostridium difficile infection after coronary artery bypass graft surgery. The Journal of Thoracic and Cardiovascular Surgery 151:2, 589-597.e2. [Crossref]

101. Lisa J. Herrinton, Neal H. Shorstein, John F. Paschal, Liyan Liu, Richard Contreras, Kevin L. Winthrop, William J. Chang, Ronald B. Melles, Donald S. Fong. 2016. Comparative Effectiveness of Antibiotic Prophylaxis in Cataract Surgery. Ophthalmology 123:2, 287-294. [Crossref]

102. Christopher Pool, Jason Kass, John Spivack, Nadav Nahumi, Mohemmed Khan, Lenard Babus, Marita S. Teng, Eric M. Genden, Brett A. Miles. 2016. Increased Surgical Site Infection Rates following Clindamycin Use in Head and Neck Free Tissue Transfer. Otolaryngology-Head and Neck Surgery 154:2, 272-278. [Crossref] 
103. Shitanshu Uppal, John Harris, Ahmed Al-Niaimi, Carolyn W. Swenson, Mark D. Pearlman, R. Kevin Reynolds, Neil Kamdar, Ali Bazzi, Darrell A. Campbell, Daniel M. Morgan. 2016. Prophylactic Antibiotic Choice and Risk of Surgical Site Infection After Hysterectomy. Obstetrics \& Gynecology 127:2, 321-329. [Crossref]

104. Joe Pardo, Kenneth P. Klinker, Barbara Swift, Samuel J. Borgert, Nikolaus Gravenstein. 2016. Moving Closer to Zero: Effect of "Just in Time" Dosing of Prophylactic Antibiotics on Surgical Site Infections. Hospital Pharmacy 51:1, 44-48. [Crossref]

105. Michelle K. Rhee, Francis S. Mah. 2016. Cataract Drug Delivery Systems (Dropless vs. Nondropless Cataract Surgery). International Ophthalmology Clinics 56:3, 117-136. [Crossref]

106. Robert G. Martindale, Clifford W. Deveney. Preoperative Preparation of the Patient Undergoing Incisional Hernia Repair: Optimizing Chances for Success 31-40. [Crossref]

107. Itzhak Brook. 2016. Spectrum and treatment of anaerobic infections. Journal of Infection and Chemotherapy 22:1, 1-13. [Crossref]

108. Thomas Curran, Vitaliy Poylin, Deborah Nagle. 2016. Real world dehiscence rates for patients undergoing abdominoperineal resection with or without myocutaneous flap closure in the national surgical quality improvement project. International Journal of Colorectal Disease 31:1, 95-104. [Crossref]

109. Addison K. May. Soft Tissue Infections 281-293. [Crossref]

110. Chierichini Angelo, Santoprete Stefano, Frassanito Luciano. Anesthesia and Perioperative Care in Cervical Spine Surgery 57-72. [Crossref]

111. Bo Young Park, Jung Woo Kwon, So Ra Kang, Seung Eun Hong. 2016. Analysis of Malpractice Claims Associated with Surgical Site Infection in the Field of Plastic Surgery. Journal of Korean Medical Science 31:12, 1963. [Crossref]

112. Ali Akhaddar. Preventive Measures Against Cranial Bone Infection 311-317. [Crossref]

113. Sébastien Bailly, Danièle Maubon, Pierre Fournier, Hervé Pelloux, Carole Schwebel, Claire Chapuis, Luc Foroni, Muriel Cornet, Jean-François Timsit. 2016. Impact of antifungal prescription on relative distribution and susceptibility of Candida spp. - Trends over 10 years. Journal of Infection 72:1, 103-111. [Crossref]

114. Lena M. Napolitano. Acute Soft Tissue and Bone Infections 351-370. [Crossref]

115. Ohashi Masaki, Saka Makoto, Katayama Hiroshi, Okinaka Keiji, Morita Shinji, Fukagawa Takeo, Katai Hitoshi. 2015. A Prospective Cohort Study To Evaluate the Feasibility of Intraoperative Antimicrobial Prophylaxis in Open Gastrectomy for Gastric Cancer. Surgical Infections 16:6, 833-839. [Abstract] [Full Text HTML] [Full Text PDF] [Full Text PDF with Links]

116. Alexis Elward, Jeanne Yegge, Angela Recktenwald, Louise Jadwisiak, Patti Kieffer, Melinda Hohrein, Diane Hopkins-Broyles, Keith F. Woeltje. 2015. Risk Factors for Craniotomy or Spinal Fusion Surgical Site Infection. The Pediatric Infectious Disease Journal 34:12, 1323-1328. [Crossref]

117. Katherine C. Hubert. 2015. Commentary to 'Post-surgical infections and perioperative antibiotics usage in pediatric genitourinary procedures'. Journal of Pediatric Urology 11:6, 359. [Crossref]

118. Zachary M. Dong, Alexis P. Chidi, Julie Goswami, Katrina Han, Richard L. Simmons, Matthew R. Rosengart, Allan Tsung. 2015. Prior inpatient admission increases the risk of post-operative infection in hepatobiliary and pancreatic surgery. $H P B$ 17:12, 1105-1112. [Crossref]

119. Chee S. Wong, Grainne Cousins, John C. Duddy, Stewart R. Walsh. 2015. Intra-abdominal drainage for laparoscopic cholecystectomy: A systematic review and meta-analysis. International Journal of Surgery 23, 87-96. [Crossref]

120. Brown Jennifer, Li Chin-Shang, Giordani Mauro, Shahlaie Kiarash, Klineberg Eric O., Tripet-Diel Joanna R., Ihara Marie S., Cohen Stuart H.. 2015. Swabbing Surgical Sites Does Not Improve the Detection of Staphylococcus aureus Carriage in High-Risk Surgical Patients. Surgical Infections 16:5, 523-525. [Abstract] [Full Text HTML] [Full Text PDF] [Full Text PDF with Links]

121. MM Marican, SM Fook-Chong, IS Rikhraj. 2015. Incidence of postoperative wound infections after open tendo Achilles repairs. Singapore Medical Journal 56:10, 549-554. [Crossref]

122. Cynthia Argani, Evie Notis, Rachel Moseley, Kerri Huber, Scott Lifchez, Leigh Ann Price, Jonathan Zenilman, Andrew Satin, Trish M. Perl, Geetika Sood. 2015. Survey of Cesarean Delivery Infection Prevention Practices Across US Academic Centers. Infection Control \& Hospital Epidemiology 36:10, 1245-1247. [Crossref]

123. Ashraf F Nabhan, Nahed E Allam, Mohamed Hamed Abdel-Aziz Salama. Routes of administration of antibiotic prophylaxis for preventing infection after caesarean section . [Crossref]

124. Darwin Ang, Mark McKenney, Scott Norwood, Stanley Kurek, Brian Kimbrell, Huazhi Liu, Michele Ziglar, James Hurst. 2015. Benchmarking statewide trauma mortality using Agency for Healthcare Research and Quality's patient safety indicators. Journal of Surgical Research 198:1, 34-40. [Crossref]

125. Elizabeth C. Wick, Daniel J. Galante, Deborah B. Hobson, Andrew R. Benson, K.H. Ken Lee, Sean M. Berenholtz, Jonathan E. Efron, Peter J. Pronovost, Christopher L. Wu. 2015. Organizational Culture Changes Result in Improvement in Patient-Centered 
Outcomes: Implementation of an Integrated Recovery Pathway for Surgical Patients. Journal of the American College of Surgeons 221:3, 669-677. [Crossref]

126. Joseph A. Bosco, Jared Bookman, James Slover, Emmanuel Edusei, Brett Levine. 2015. Principles of Antibiotic Prophylaxis in Total Joint Arthroplasty. Journal of the American Academy of Orthopaedic Surgeons 23:8, e27-e35. [Crossref]

127. Veerajalandhar Allareddy, Ashima Das, Min Kyeong Lee, Romesh P. Nalliah, Sankeerth Rampa, Veerasathpurush Allareddy, Alexandre T. Rotta. 2015. Prevalence, predictors, and outcomes of methicillin-resistant Staphylococcus aureus infections in patients undergoing major surgical procedures in the United States: a population-based study. The American Journal of Surgery 210:1, 59-67. [Crossref]

128. Jose P. Zevallos. 2015. An Administrative Data Approach to Examining Perioperative Antibiotic Use in Head and Neck Oncologic Surgery. Otolaryngology-Head and Neck Surgery 153:1, 69-70. [Crossref]

129. Hibbert Denise, Abduljabbar Alaa S., Alhomoud Samar J., Ashari Luai H., Alsanea Nasser. 2015. Risk Factors for Abdominal Incision Infection after Colorectal Surgery in a Saudi Arabian Population: The Method of Surveillance Matters. Surgical Infections 16:3, 254-262. [Abstract] [Full Text HTML] [Full Text PDF] [Full Text PDF with Links]

130. Dawn Merton Boothe, Harry W. Boothe. 2015. Antimicrobial Considerations in the Perioperative Patient. Veterinary Clinics of North America: Small Animal Practice 45:3, 585-608. [Crossref]

131. Eduardo Asín-Prieto, Amaia Soraluce, Iñaki F. Trocóniz, Eugenia Campo Cimarras, Jaione Sáenz de Ugarte Sobrón, Alicia Rodríguez-Gascón, Arantxazu Isla. 2015. Population pharmacokinetic models for cefuroxime and metronidazole used in combination as prophylactic agents in colorectal surgery: Model-based evaluation of standard dosing regimens. International Journal of Antimicrobial Agents 45:5, 504-511. [Crossref]

132. Lindsay Maggio, David P. Nicolau, Melissa DaCosta, Dwight J. Rouse, Brenna L. Hughes. 2015. Cefazolin Prophylaxis in Obese Women Undergoing Cesarean Delivery. Obstetrics \& Gynecology 125:5, 1205-1210. [Crossref]

133. Ruthy Tal Jasper, Joseph R Coyle, David E Katz, Dror Marchaim. 2015. The complex epidemiology of extended-spectrum $\beta$ lactamase-producing Enterobacteriaceae. Future Microbiology 10:5, 819-839. [Crossref]

134. Kenji L. Leonard, Stephen W. Davies, Brett H. Waibel. 2015. Perioperative Management of Obese Patients. Surgical Clinics of North America 95:2, 379-390. [Crossref]

135. Jisha John, Kyle Miletic, Keith S. Kaye. 2015. Skin and Skin Structure Infections in Older Adults. Current Geriatrics Reports 4:1, 70-78. [Crossref]

136. Philip S. Barie, Samuel E. Wilson. 2015. Impact of Evolving Epidemiology on Treatments for Complicated Skin and Skin Structure Infections: The Surgical Perspective. Journal of the American College of Surgeons 220:1, 105-116.e6. [Crossref]

137. 2015. Rhinosinusitis: evidence and experience. Brazilian Journal of Otorbinolaryngology 81, S1-S49. [Crossref]

138. Sangeeta Sastry, Riaz Rahman, Mohamed H. Yassin. 2015. Cardiac Implantable Electronic Device Infection: From an Infection Prevention Perspective. Advances in Preventive Medicine 2015, 1-8. [Crossref]

139. Melissa E. Harding. 2015. Cardiac Implantable Electronic Device Implantation. AACN Advanced Critical Care 26:4, 312-319. [Crossref]

140. Alyssa D Throckmorton, Amy C Degnim. 2015. Infections after breast surgery: potential ways to reduce infection rates. Breast Cancer Management 4:1, 17-24. [Crossref]

141. Greta L. Piper, Adrian A. Maung. Perioperative Probiotics 1025-1034. [Crossref]

142. Pagovich Odelya E., Lebastchi Amir H., Romberg Neil. 2014. Peri-Operative Considerations in the Patient with Primary Immune Deficiency: A Review. Surgical Infections 15:6, 672-678. [Abstract] [Full Text HTML] [Full Text PDF] [Full Text PDF with Links]

143. O. Obinwa, M. Casidy, J. Flynn. 2014. The microbiology of bacterial peritonitis due to appendicitis in children. Irish Journal of Medical Science (1971 -) 183:4, 585-591. [Crossref]

144. Rebecca F. Turcotte, Ava Brozovich, Rozelle Corda, Ryan T. Demmer, Katherine V. Biagas, Diane Mangino, Lisa Covington, Anne Ferris, Brian Thumm, Emile Bacha, Art Smerling, Lisa Saiman. 2014. Health Care-Associated Infections in Children After Cardiac Surgery. Pediatric Cardiology 35:8, 1448-1455. [Crossref]

145. Brandy L. Edwards, George J. Stukenborg, David R. Brenin, Anneke T. Schroen. 2014. Use of Prophylactic Postoperative Antibiotics During Surgical Drain Presence Following Mastectomy. Annals of Surgical Oncology 21:10, 3249-3255. [Crossref]

146. S.M. Rehman, O. Elzain, J. Mitchell, B. Shine, I.C.J.W. Bowler, R. Sayeed, S. Westaby, C. Ratnatunga. 2014. Risk factors for mediastinitis following cardiac surgery: the importance of managing obesity. Journal of Hospital Infection 88:2, 96-102. [Crossref] 
147. Maha Talaat, Tamer Saied, Amr Kandeel, Gehad El-Ata, Amani El-Kholy, Soad Hafez, Ashraf Osman, Mohamed Razik, Ghada Ismail, Sherine El-Masry, Rami Galal, Mohamad Yehia, Amira Amer, David Calfee. 2014. A Point Prevalence Survey of Antibiotic Use in 18 Hospitals in Egypt. Antibiotics 3:3, 450-460. [Crossref]

148. Unger Nathan R., Stein Bradley J.. 2014. Effectiveness of Pre-Operative Cefazolin in Obese Patients. Surgical Infections 15:4, 412-416. [Abstract] [Full Text HTML] [Full Text PDF] [Full Text PDF with Links]

149. Pascal M Dohmen, Martin Misfeld, Michael A Borger, Friedrich W Mohr. 2014. Closed incision management with negative pressure wound therapy. Expert Review of Medical Devices 11:4, 395-402. [Crossref]

150. Susan Kline, Maya Highness, Loreen A. Herwaldt, Trish M. Perl. 2014. Variable Screening and Decolonization Protocols for Staphylococcus aureus Carriage Prior to Surgical Procedures. Infection Control \& Hospital Epidemiology 35:07, 880-882. [Crossref]

151. Laura Armañanzas, Jaime Ruiz-Tovar, Antonio Arroyo, Pedro García-Peche, Ernesto Armañanzas, María Diez, Isabel Galindo, Rafael Calpena. 2014. Prophylactic Mesh vs Suture in the Closure of the Umbilical Trocar Site after Laparoscopic Cholecystectomy in High-Risk Patients for Incisional Hernia. A Randomized Clinical Trial. Journal of the American College of Surgeons 218:5, 960-968. [Crossref]

152. E. Tan, N. Mortimer, P. Salmon. 2014. Full-thickness skin grafts for surgical defects of the nasal ala - a comprehensive review, approach and outcomes of 186 cases over 9 years. British Journal of Dermatology 170:5, 1106-1113. [Crossref]

153. Melissa E. Harding, Larry A. Chinitz. 2014. Clinical considerations for allied professionals: Optimizing outcomes: Surgical incision techniques and wound care in device implantation. Heart Rhytbm 11:4, 737-741. [Crossref]

154. Yi Zhang, Jiasheng Dong, Yufei Qiao, Jinguang He, Tao Wang, Sunxiang Ma. 2014. Efficacy and Safety Profile of Antibiotic Prophylaxis Usage in Clean and Clean-Contaminated Plastic and Reconstructive Surgery. Annals of Plastic Surgery 72:1, 121-130. [Crossref]

155. Robert W. Krell, Micah E. Girotti, Danielle Fritze, Darrell A. Campbell, Samantha Hendren. 2013. Hospital Readmissions after Colectomy: A Population-Based Study. Journal of the American College of Surgeons 217:6, 1070-1079. [Crossref]

156. Jason D. Wright, Khalid Hassan, Cande V. Ananth, Thomas J. Herzog, Sharyn N. Lewin, William M. Burke, Yu-Shiang Lu, Alfred I. Neugut, Dawn L. Hershman. 2013. Use of Guideline-Based Antibiotic Prophylaxis in Women Undergoing Gynecologic Surgery. Obstetrics \& Gynecology 122:6, 1145-1153. [Crossref]

157. Francesco Napolitano, Maria Teresa Izzo, Gabriella Di Giuseppe, Italo F. Angelillo. 2013. Evaluation of the Appropriate Perioperative Antibiotic Prophylaxis in Italy. PLoS ONE 8:11, e79532. [Crossref]

158. E. Launay, V. Pichenot, R. Dumont, J. Caillon, C. Gras-Le Guen. 2013. Antibioprophylaxie en chirurgie orthopédique pédiatrique. Archives de Pédiatrie 20, S74-S78. [Crossref]

159. R. Dumont, V. Pichenot, C. Gras-Le Guen, E. Launay. 2013. Antibioprophylaxie chirurgicale : les grands principes, état des lieux et exemple d'harmonisation des pratiques. Archives de Pédiatrie 20, S61-S66. [Crossref]

160. Robert G. Martindale, Clifford W. Deveney. 2013. Preoperative Risk Reduction. Surgical Clinics of North America 93:5, 1041-1055. [Crossref]

161. Charles de Mestral, Avery B. Nathens. 2013. Prevention, Diagnosis, and Management of Surgical Site Infections. Critical Care Clinics 29:4, 887-894. [Crossref]

162. Natasha Bagdasarian, Kenneth E. Schmader, Keith S. Kaye. 2013. The Epidemiology and Clinical Impact of Surgical Site Infections in the Older Adult. Current Translational Geriatrics and Experimental Gerontology Reports 2:3, 159-166. [Crossref]

163. Philip S. Barie. 2013. Guidelines for Antimicrobial Prophylaxis in Surgery: A Must-Read, Must-Heed for Every Surgeon. Surgical Infections 14:1, 5-7. [Citation] [Full Text HTML] [Full Text PDF] [Full Text PDF with Links]

164. Ellen A. Kim. 2013. Methicillin-resistant Staphylococcus aureus (MRSA) Infection in Neonates. Neonatal Medicine 20:3, 354. [Crossref] 